\title{
A PRELIMINARY INVESTIGATION OF MARINE YEAST BIODIVERSITY IN NEW ZEALAND WATERS
}

BY

\section{MELISSA FRANCIS}

\begin{abstract}
A thesis
submitted to the Victoria University of Wellington

in fulfilment of the requirements for the degree of

Master of Science
\end{abstract}

Victoria University of Wellington

2013 





\section{Abstract}

This is the first known investigation of marine yeast biodiversity from waters surrounding New Zealand's main Islands. Marine yeasts were cultured onto agar plates from algae sampled at three locations in the Wellington Region. DNA extractions and PCR amplifications of the internal transcribed spacer (ITS) regions were conducted, and resultant sequence data were used for isolate identification and phylogenetic analysis. Yeasts isolated during this investigation were not unique; seventy-four isolates were identified from a range of genera that are frequently detected in marine and terrestrial environments worldwide. Furthermore, high ITS sequence similarity was observed between yeasts isolated during this investigation and those from geographically distant locations. These findings may indicate that marine yeasts are ubiquitous at a global level, although evidence is insufficient as to whether yeasts also demonstrate biogeographic distribution patterns. Yeasts isolated during this investigation may have ecological implications in New Zealand's marine environment; marine yeasts are likely to play a general saprophytic role and certain genera are pathogenic. Isolates were also identified from genera that have previously demonstrated beneficial properties and applications, including the production of useful compounds and highly nutritious yeast biomass, biocontrol potential against the postharvest decay of produce, and degradation abilities that may enable bioremediation of polluted marine environments. 



\section{Acknowledgements}

I would like to express my gratitude to my supervisors Vicky Webb and Joe Zuccarello. I would also like to thank the following people for their advice and technical assistance: Sally Anderson, Malcolm Francis, Erasmo Macaya Horta, Margaret McVeagh, Kate Neill, Wendy Nelson, Caoimhghin O’Maolagain, Peter Ritchie, Peter Smith and Matt Voyles. 



\section{Table of Contents}

1. Introduction ......................................................................................................... 1

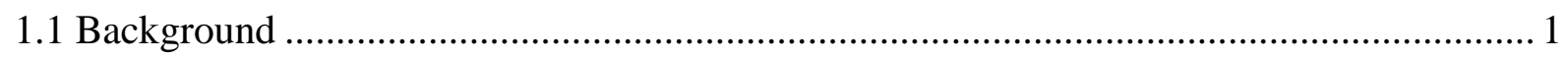

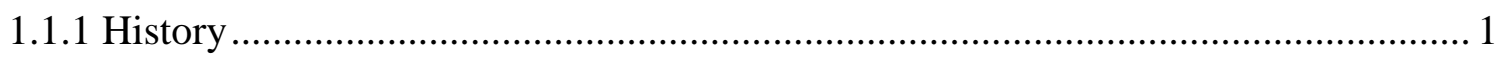

1.1.2 Marine Yeasts versus Terrestrial Yeasts ............................................................... 2

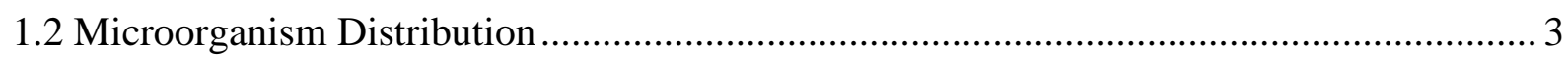

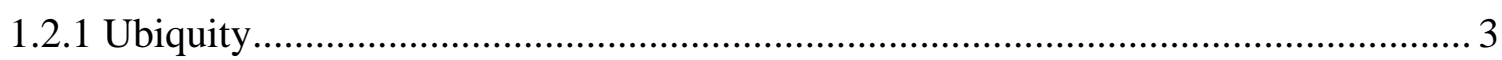

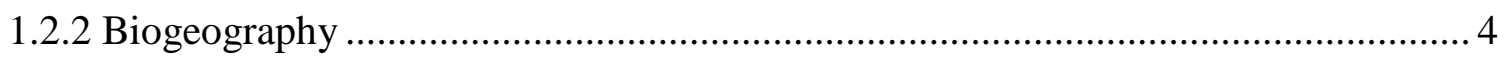

1.2.3 A Compromise between Ubiquity and Biogeography ...................................... 5

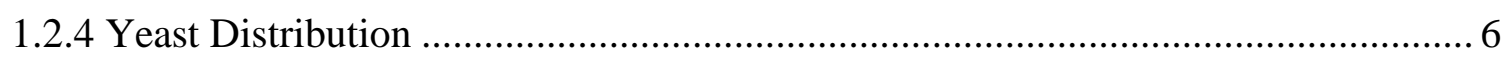

1.3 Fungal Systematics and Molecular Techniques ....................................................... 8

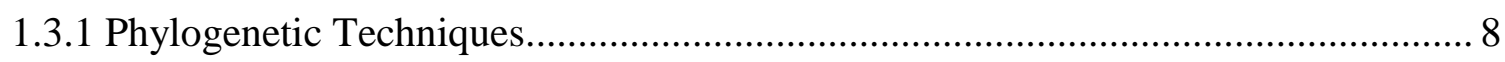

1.3.2 Internal Transcribed Spacer Region ............................................................ 9

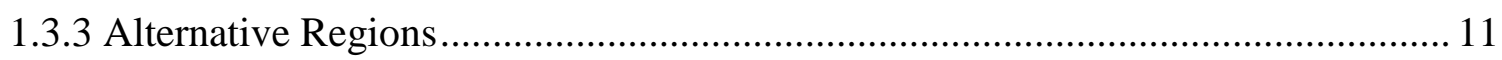

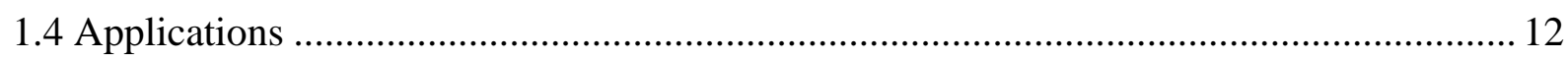

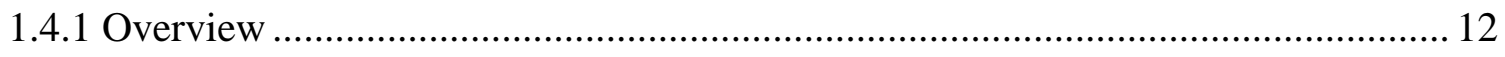

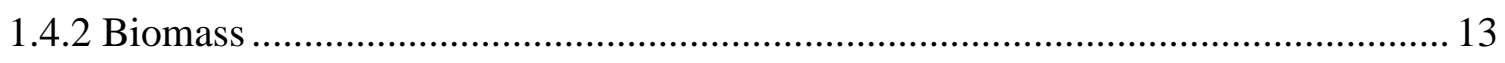

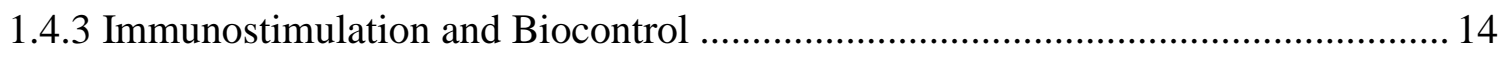

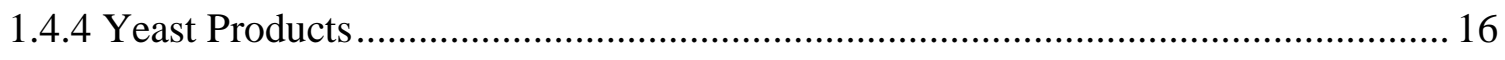

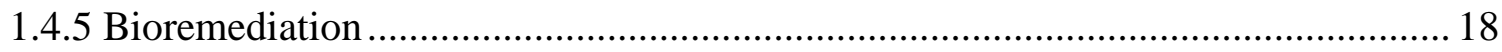




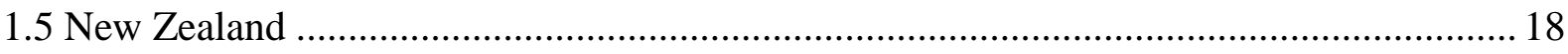

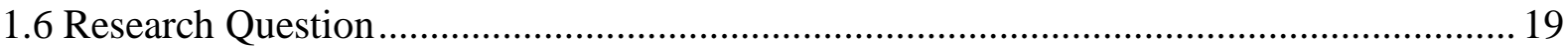

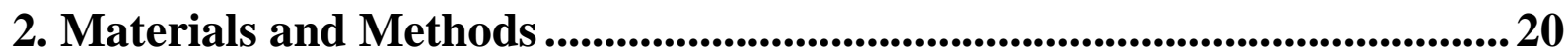

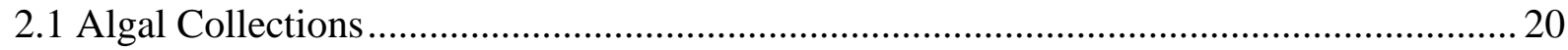

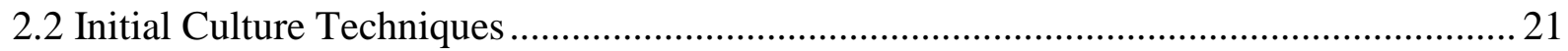

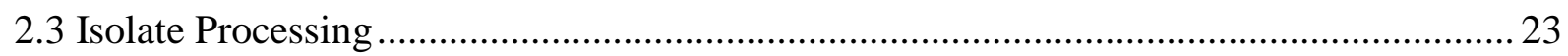

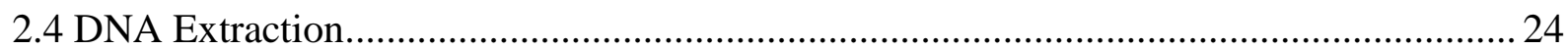

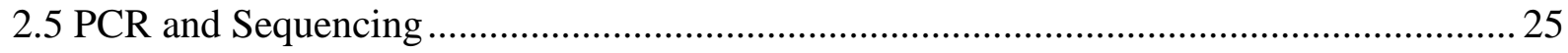

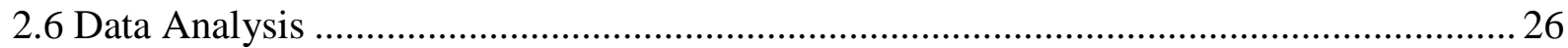

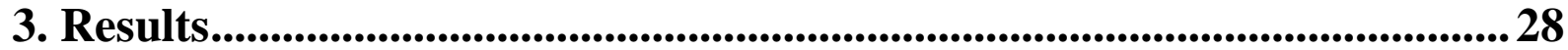

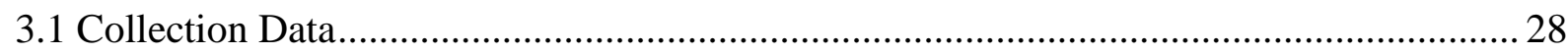

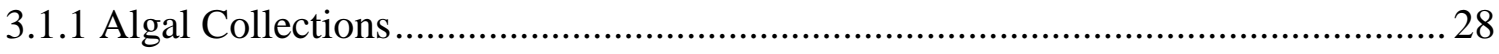

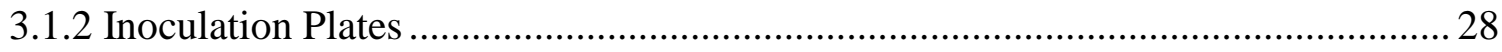

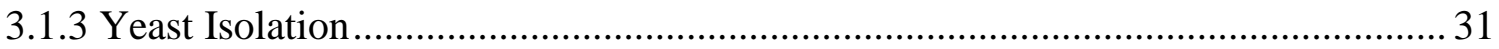

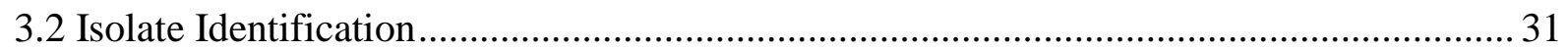

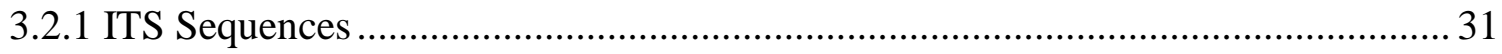

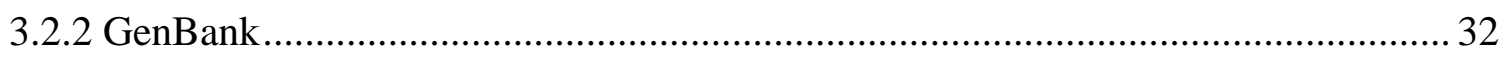

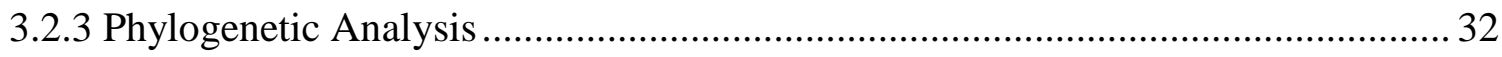

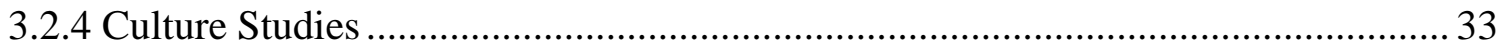

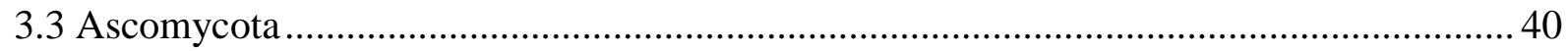

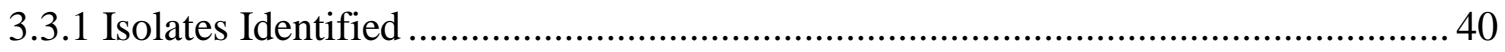

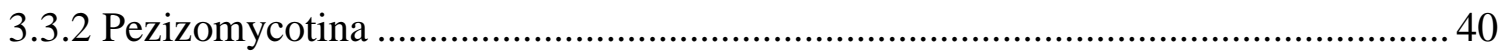


3.3.2.1 Identification via Molecular Techniques....................................................... 40

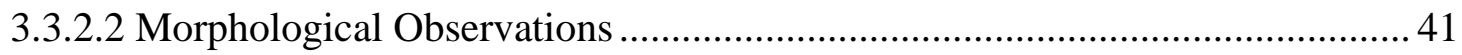

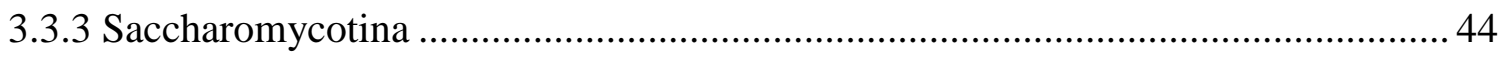

3.3.3.1 Identification via Molecular Techniques................................................ 44

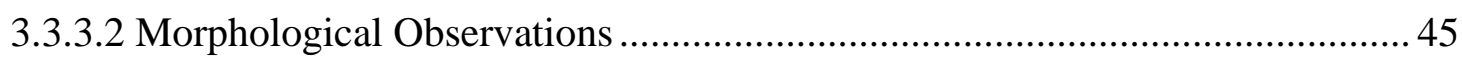

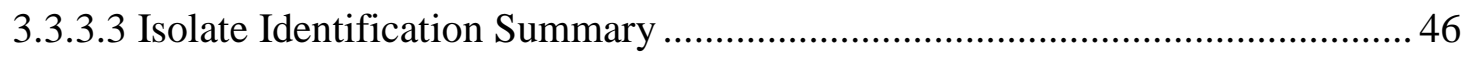

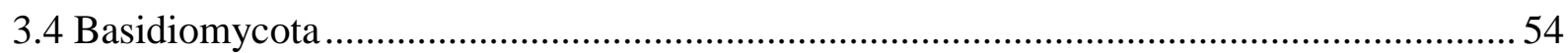

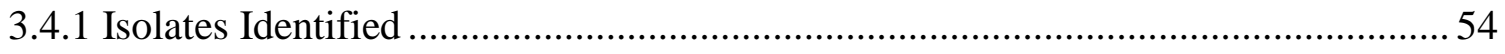

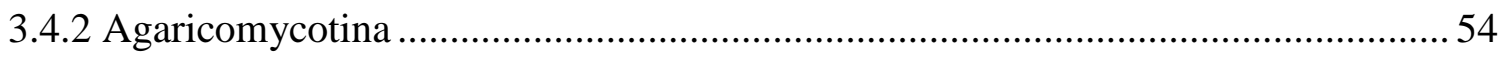

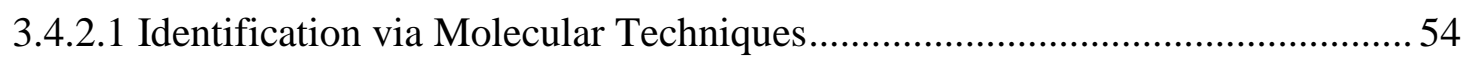

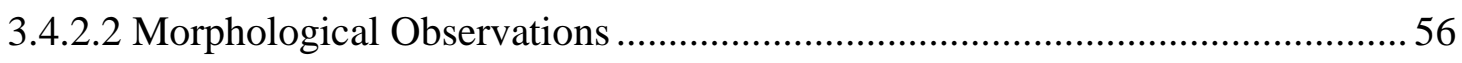

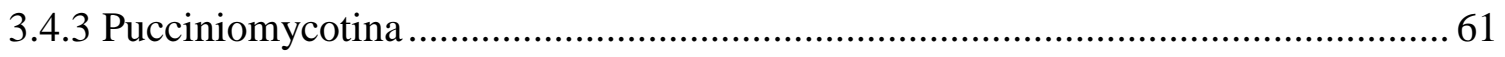

3.4.3.1 Identification via Molecular Techniques................................................... 61

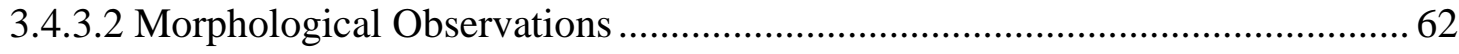

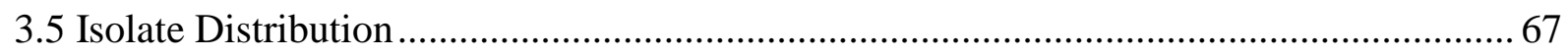

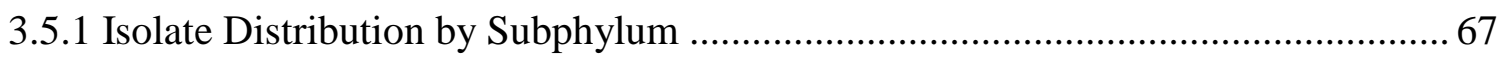

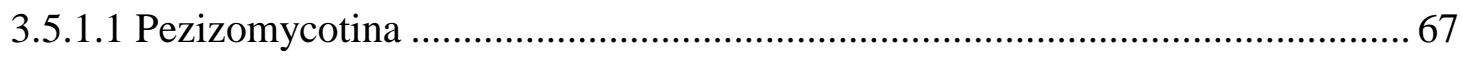

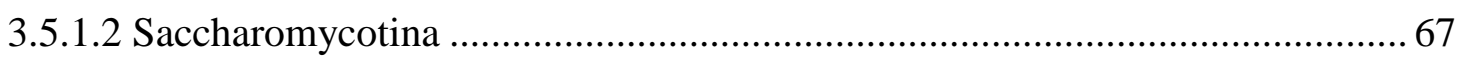

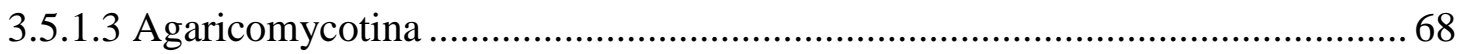

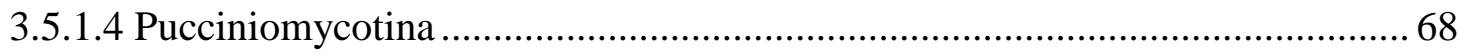

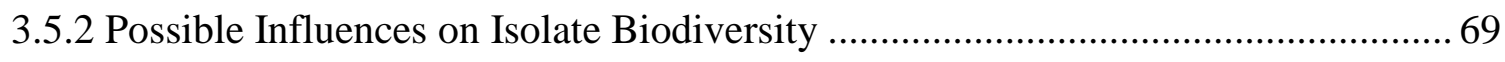

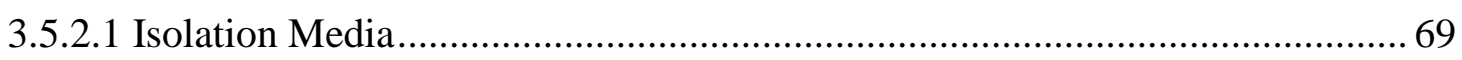

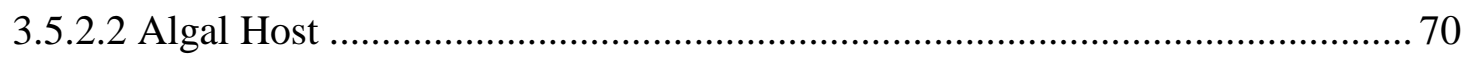

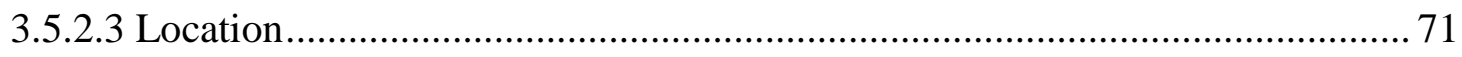




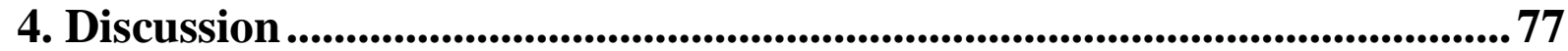

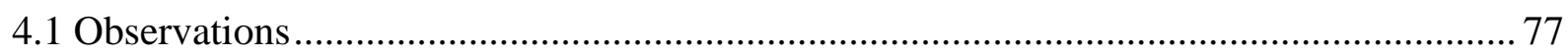

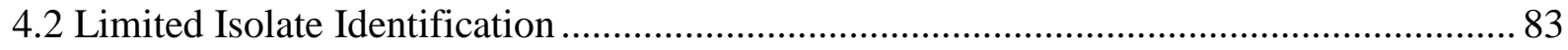

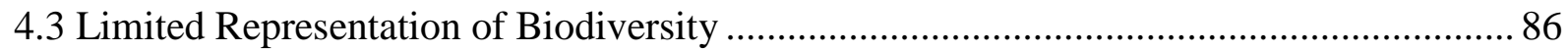

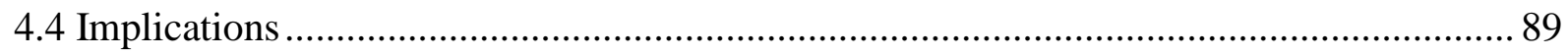

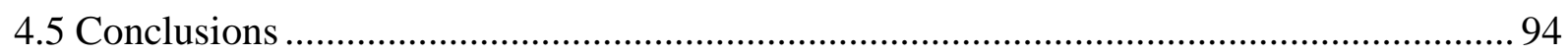

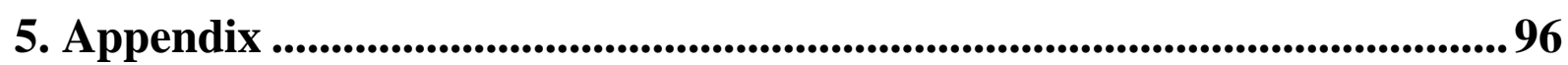

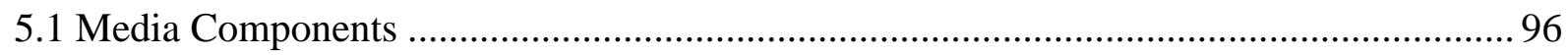

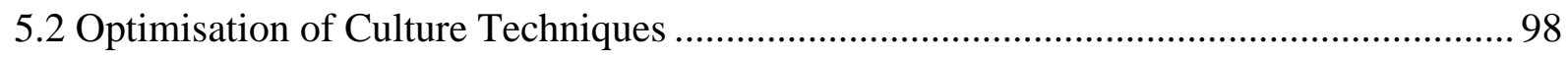

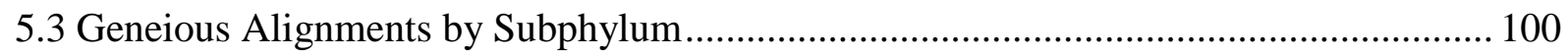

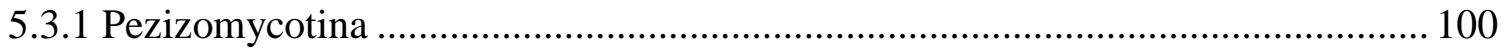

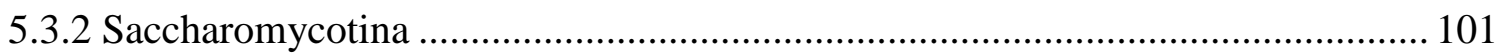

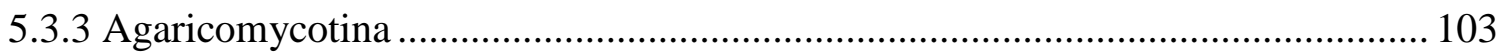

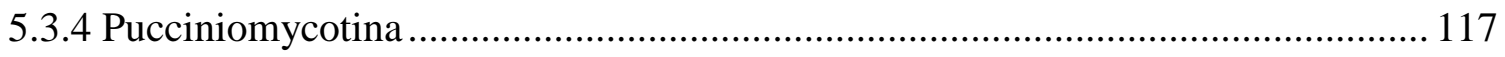

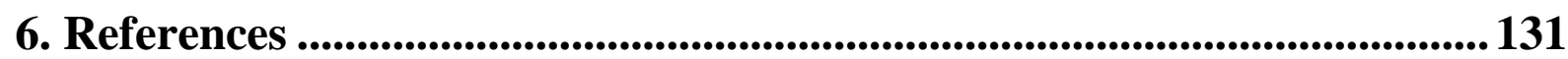




\section{Table of Figures}

Figure 1. Map illustrating the location of algal collections conducted in the Wellington Region.

Figure 2. Photographs illustrating the appearance of agar plates after, inoculation with yeasts from algae and one week of incubation at $18^{\circ} \mathrm{C}$. 30

Figure 3. Photographs of colonies formed by Pezizomycotina isolates on agar plates, and microscopic images of the corresponding isolates.

Figure 4. Neighbour-joining phylogenetic tree based on ITS sequences from Saccharomycotina isolates, using the Tamura-Nei model. .47

Figure 5. Photographs of colonies formed by Saccharomycotina isolates on agar plates. ..... 52

Figure 6. Microscopic images of Saccharomycotina isolates.

Figure 7. Neighbour-joining phylogenetic tree based on ITS sequences from Agaricomycotina isolates, using the Tamura-Nei model. .57

Figure 8. Photographs of colonies formed by Agaricomycotina isolates on agar plates.

Figure 9. Microscopic images of Agaricomycotina isolates. 60

Figure 10. Neighbour-joining phylogenetic tree based on ITS sequences from Pucciniomycotina isolates, using the Tamura-Nei model.

Figure 11. Photographs of colonies formed by Pucciniomycotina isolates on agar plates. .... 65

Figure 12. Microscopic images of Pucciniomycotina isolates. 66

Figure 13. Pie graphs illustrating the isolation frequency of different yeast genera during four algal collections. .74

Figure 14. Summary of isolates identified at three taxonomic levels. .75 


\section{Table of Tables}

Table 1. Particulars regarding algae sampled in the Wellington Region.

Table 2. Isolate identification based on GenBank search results and phylogenetic analysis using ITS sequence data. 34

Table 3. Morphology of Pezizomycotina isolates.

Table 4. Morphology of Saccharomycotina isolates. .48

Table 5. Morphology of Agaricomycotina isolates. .58

Table 6. Morphology of Pucciniomycotina isolates. 64

Table 7. Isolation frequency of different yeast genera on two types of media. .72

Table 8. Isolation frequency of different yeast genera from ten algal species. .73

Table 9. Appearance of agar plates after inoculation with seawater or the solution resulting from homogenisation of an algal sample in sterile seawater. .99

Table 10. Appearance of agar plates after treatment with antibiotics and inoculation with the solution resulting from homogenisation of an algal sample in sterile seawater. .99 




\section{Introduction}

\subsection{Background}

\subsubsection{History}

Yeasts are unicellular, eukaryotic microorganisms, consisting of two phyla in the kingdom Fungi: Ascomycota and Basidiomycota (Kutty and Philip 2008). Yeasts have historical significance in most societies worldwide due to their role in the production of fermented food and beverages (Legras et al. 2007). A letter written by Antony van Leeuwenhoek in 1680 described yeast cells in beer and is the first known record of their observation (Dobell 1932). Current literature searches reveal vast amounts of research on terrestrial yeast species, in particular Saccharomyces cerevisiae Meyen ex E.C. Hansen which is a useful model for the study of eukaryotic cell biology (Botstein et al. 1997). In comparison, few studies have focused on marine yeasts; most investigations from the 1940's and earlier were either incidental or provided incomplete information for yeast identification (Fell et al. 1960). Nevertheless, irregular accounts and descriptions of marine fungi exist from as early as 1850 (Kohlmeyer 1963). The first known record of marine yeasts was not until 1894 when Fischer isolated yeasts from waters of the Atlantic Ocean, the majority of which were identified as white and red “Torula" species (Phaff et al. 1952). By the mid 1960's, several researchers had isolated over 30 different yeast species from various marine environments (Fell and Van Uden 1963). Yeasts from both the Ascomycota and the Basidiomycota were isolated in early studies, even though it was originally thought that the sea was nearly devoid of the 
Basidiomycota (Wilson 1960). For example, multiple species from the genera Candida (Ascomycota) and Rhodotorula (Basidiomycota) were isolated from Biscayne Bay, Florida (Fell et al. 1960). Recent investigations have isolated yeasts from a wide range of marine substrates and habitats, but many locations have yet to be surveyed (Kutty and Philip 2008).

\subsubsection{Marine Yeasts versus Terrestrial Yeasts}

Yeasts considered to have terrestrial origins have been regularly isolated from marine and brackish environments (Fell et al. 1960; Singh et al. 2010). This has raised questions: what distinguishes marine yeasts from terrestrial ones? are yeasts isolated from marine environments obligate marine forms, or terrestrial contaminants passively surviving in the sea? (Van Uden and Castelo Branco 1963; Kohlmeyer and Kohlmeyer 1979). Marine fungi are adaptable (Kohlmeyer 1963), so the answers to these two questions are not obvious. Genetically, there is no specific group that makes up the marine yeasts; yeasts from marine and terrestrial sources are distributed over many genera, such as Candida and Debaryomyces (Kurtzman 1994). Physiologically, no defining characteristics are known to explain the habitation of marine yeasts in marine environments (Kohlmeyer and Kohlmeyer 1979). The majority of marine yeasts isolated during one investigation were physiologically similar to their terrestrial counterparts (Fell et al. 1960). Rhodotorula species from marine and terrestrial sources were also similar in terms of their metabolic attributes, although exceptions such as the additional nutrients required by marine strains of Rhodotorula aurantiaca (Saito) Lodder occurred in some situations (Ahearn et al. 1962). Salinity tolerance is not exclusive to marine yeasts, as the growth of most species is not inhibited by sodium chloride concentrations greater than those of the ocean (Kohlmeyer and Kohlmeyer 1979). Since yeasts isolated from 
marine sources generally grow equally well on media prepared with saltwater or freshwater, they cannot be distinguished as marine or terrestrial simply based on which media elicits optimal growth (Fell and Van Uden 1963). Overall, currently available information does not enable straightforward differentiation of marine and terrestrial yeasts, or great improvement to Kohlmeyer's original definition of marine fungi as "those that grow and reproduce submerged in the sea or on the seashore on intertidal substrates" (Kohlmeyer 1963).

\subsection{Microorganism Distribution}

\subsubsection{Ubiquity}

There is currently debate as to whether microorganisms are ubiquitous, or if they exhibit biogeographic patterns (Martiny et al. 2006). Early studies by Baas Becking and Beijerinck shaped concepts that are still relevant when studying microorganism biogeography and Baas Becking's original theory from 1934, "everything is everywhere, but, the environment selects", is commonly cited (Baas Becking 1934; De Wit and Bouvier 2006). There is suggestion and evidence that microbial eukaryotes are generally cosmopolitan, and do not have biogeographies (Finlay 2002; Finlay and Fenchel 2004). The small physical size and huge abundance of microorganisms raises the probability that they may be transported over large distances simply by chance (Finlay and Esteban 2001). Dispersal of microorganisms is thought to occur via essentially random forces, including both physical means such as ocean currents and hurricanes, and biological means such as transport via other organisms (Finlay 2002). Dispersal may result in the introduction of microorganisms to new locations, where 
their subsequent colonisation could overwhelm any tendency towards genetic differentiation (Martiny et al. 2006). However, random dispersal does not necessarily result in relocation of microorganisms to their preferred habitat. One suggestion is that microorganisms are generally ubiquitous and will grow wherever their preferred habitat is realised (Boenigk et al. 2006, plus references therein). A ubiquitous seed bank may exist, in which a large reservoir of microbial species occur in a given environment; some microorganisms may thrive and others may persist only as spores or cysts (Finlay and Esteban 2001). This theory implies the existence of a pool of undetected microorganisms and that local extinction of specific species is rare (Fenchel and Finlay 2004). The number of microbial species worldwide would be small relative to local species richness (Finlay 2002), and ecosystem functions would never be compromised by a lack of microbial diversity (Finlay et al. 1997). Various investigations support the theory of microorganism ubiquity; bacterial sequences from peat sampled in Germany demonstrated similarity with sequences from marine and terrestrial habitats in distant locations including New Zealand (Rheims et al. 1996). Furthermore, an increase in the proportion of cosmopolitan species was observed as organism size decreased in two habitats that have been extensively sampled for eukaryotes: Nivå Bay, an estuary-like habitat near Copenhagen; and Priest Pot, a eutrophic freshwater pond in England (Finlay and Fenchel 2004).

\subsubsection{Biogeography}

In contrast to the theory of ubiquity, certain evidence suggests that free-living microorganisms demonstrate biogeographic distribution patterns (Martiny et al. 2006). "The environment selects" is the second part of Baas Becking and Beijerinck's tenet (Baas Becking 1934; De 
Wit and Bouvier 2006), and rather than existing environmental conditions it is argued that past events or geographic factors are responsible for shaping spatial variation in microorganism diversity (Baas Becking 1934; Martiny et al. 2006). Data from various investigations support the theory that geographic barriers influence the distribution of eukaryotic microorganisms. Spumella morphospecies typically demonstrate a positive linear trend between heat tolerance and the ambient air temperature of their isolation site, however, Spumella isolates from Antarctica possessed a lower maximum tolerated temperature (Boenigk et al. 2006). These findings support the idea of restricted geographic transport; Spumella migration to Antarctica was sufficiently restricted to allow adaptation of local populations to the environmental conditions present (Boenigk et al. 2006). Minimal gene flow between populations was also observed during an investigation of the hyperthermophilic archaeon Sulfolobus (Whitaker et al. 2003). The specific growth requirements of Sulfolobus may have prevented dispersal, or perhaps immigrants were unable to persist in locations with established microorganism populations (Whitaker et al. 2003). Since current evaluations of biodiversity may overlook endemic ecophysiological traits and genomic variance, additional evidence of geographic barriers on microorganism distribution might become apparent if finer taxonomic resolution is considered (Boenigk et al. 2006; Gazis et al. 2011).

\subsubsection{A Compromise between Ubiquity and Biogeography}

Since there is supporting evidence for both theories of microorganism distribution, a potential alternative could include aspects of ubiquity and biogeography. An investigation of the freshwater heterokont alga Synura morphotaxon found that species in the Synura petersenii complex could be either endemic or cosmopolitan; neither the ubiquity nor the biogeography 
theory was individually sufficient to explain the distribution of microbial eukaryotes observed worldwide (Boo et al. 2010). It was suggested that the existing theories of microbial distribution may not be mutually exclusive, assuming the Synura petersenii complex is generally representative of non-marine microbial eukaryotes (Boo et al. 2010). One proposal is that microbial eukaryotes are mainly ubiquitous, although the possibility of some endemics is also recognised (Finlay and Esteban 2001). Even though restricted migration is thought to occur, geographic barriers to microbial eukaryotes may have resulted in some cases of endemism in Spumella; Antarctic Spumella isolates differed from the global trend in terms of their maximum tolerated temperature and were thus more strongly adapted to their environment (Boenigk et al. 2006). Despite uncertainty as to whether microorganisms follow a biogeographic distribution pattern or demonstrate ubiquity, it seems that Baas Becking and Beijerinck's original statement is still relevant; "everything is everywhere" describes the situation if microorganisms are ubiquitous, as does "the environment selects" if they demonstrate biogeography (Baas Becking 1934; De Wit and Bouvier 2006).

\subsubsection{Yeast Distribution}

The extremely widespread distribution of yeasts has been recognised from as early as 1960 (Van Uden 1960). Yeasts have been isolated from a range of environments, including terrestrial sources such as soils and aquatic sources such as rivers, lakes and oceans (Van Uden 1960, plus references therein). During early investigations of marine environments yeasts were isolated from various substrates: seawater, sediments, vegetation and vertebrates (Roth et al. 1962). By 1963, multiple authors had isolated over 30 yeast species from marine sources including isolates from both the Ascomycota and the Basidiomycota (Fell and Van 
Uden 1963). For example, Candida species (Candida tropicalis (Castell.) Berkhout and Candida parapsilosis (Ashford) Langeron \& Talice) from the Ascomycota and Rhodotorula species (Rhodotorula mucilaginosa (A. Jörg.) F.C. Harrison and Rhodotorula glutinis (Fresen.) F.C. Harrison) from the Basidiomycota were frequently isolated from Biscayne Bay, Florida (Fell et al. 1960). During an investigation of Biscayne Bay and additional marine areas, the most commonly isolated genera were Candida and Debaryomyces (from the Ascomycota), plus Trichosporon, Rhodotorula and Cryptococcus (from the Basidiomycota) (Roth et al. 1962). In California, Metschnikowia zobellii (Van Uden \& Cast.-Branco) Van Uden and Candida pulcherrima (Lindner) Windisch were isolated from beach cast algae (Macrocystis pyrifera (L.) C. Agardh) (Van Uden and Castelo Branco 1963). Further sampling from coastal USA yielded an assortment of marine yeast genera similar to those isolated from the coast of Portugal at around the same time (Taysi and Van Uden 1964). Although some regions have not been extensively sampled, recent evidence suggests that marine yeasts have a widespread distribution; yeasts from a range of genera (including Candida, Cryptococcus, Debaryomyces and Rhodotorula) have been isolated from various substrates in marine and estuarine waters worldwide (Kutty and Philip 2008, plus references therein).

Although microorganisms are generally ubiquitous, existing environmental factors may also influence microbial distribution. The availability and concentration of organic material is known to affect the character of yeast populations (Fell and Van Uden 1963), as are environmental conditions such as temperature, light, pressure and $\mathrm{pH}$ (Dix and Webster 1995; Deák 2006). Terrestrial runoff and pollution are also influential on the character of yeast populations in coastal areas (Fell and Van Uden 1963; Hagler and Mendonça-Hagler 1981). An investigation of both polluted and unpolluted waters in Rio de Janeiro found an increase in 
the number and variation in the type of yeasts isolated in polluted areas; pink yeasts made up a lower proportion of the total number of yeasts isolated in polluted water (Hagler and Mendonça-Hagler 1981). However, it is difficult to distinguish whether the influence of pollution is due to the introduction of terrestrial yeasts into marine environments, the effect of pollution on yeasts already present in the environment, or both. Factors such as thermoclines, eddies, plankton blooms and surface slicks also influence yeast distribution (Gadanho et al. 2003). Furthermore, an increase in water depth or distance from land is generally associated with a decrease in the size of marine yeast populations (Gadanho et al. 2003). The low organic content of sediments may also contribute to the high proportion of oxidative yeasts observed in the deep sea (Fell et al. 1960). Even though marine yeasts are suggested to have a widespread distribution at a global level, existing environmental conditions may govern their numbers and distribution (Kutty and Philip 2008, plus references therein).

\subsection{Fungal Systematics and Molecular Techniques}

\subsubsection{Phylogenetic Techniques}

Fungal systematics was originally based on phenotypic characteristics, but recently developed molecular techniques have contributed DNA sequence data as an alternative source of information (Seifert 2009). Like fungi, yeasts were primarily identified using phenotypic characteristics such as morphology and physiology, however, certain tests are laborious and may give ambiguous results due to strain variability (Kurtzman and Robnett 1998). Techniques developed based on DNA-DNA reassociation, restriction fragment length 
polymorphisms (Kurtzman and Blanz 1998), and recently obtained DNA sequence data have enabled yeast classification to be based directly on evolutionary relationships (Kurtzman and Piškur 2006). Hence, the use of molecular data as the main source of information for yeast identification is increasing (Kurtzman and Robnett 1998). Analysis of sequence divergence has been used to resolve many phylogenetic relationships (Kurtzman and Fell 2006), and this novel source of information has led to major alterations in yeast taxonomy (Deák 1999). Although it is recognised that relying solely on physiological traits to establish yeast taxonomy can create errors, molecular and conventional taxonomies need not be exclusive as they may complement each other in polyphasic taxonomy (Valente et al. 1999). Macroscopic descriptions of colony colour are still helpful when describing yeasts; a pink or red colony is usually indicative of a species from the Basidiomycota, and a white colony could belong to either the Basidiomycota or the Ascomycota (Gadanho et al. 2003).

\subsubsection{Internal Transcribed Spacer Region}

Fungal species delineation is now primarily based on relatedness of the rRNA gene region; ribosomal nucleic acid sequences are of particular interest because they allow evaluation of phylogenetic and evolutionary relationships at multiple taxonomic levels (Deák 1999; Valente et al. 1999). White et al. (1990) described primers for amplification of the nuclear and mitochondrial rRNA genes in fungi. The nuclear gene component of rRNA consists of a small subunit (SSU) and a large subunit (LSU), separated by two internal transcribed spacer regions (ITS1 and ITS2) which are interrupted by a conserved 5.8S region (White et al. 1990). Henceforth, the term ITS will be used to refer to both the ITS1 and ITS2 regions, and includes the 5.8S region. The conserved areas of rRNA sequences enable alignment, and the more 
variable regions enable estimation of evolutionary relationships (Kurtzman 1994), thus, unequally conserved ITS components allow resolution at various scales (Nilsson et al. 2008). James et al. (1996) analysed ITS and SSU rRNA sequence data and found that the patterns of relationships were generally in agreement, although ITS sequences were superior for assessment of very close relationships due to greater interspecies sequence divergence. Additional advantages of the rRNA region for species identification include its occurrence in multiple copies in all living cellular organisms (Kurtzman and Piškur 2006), plus its 550 bp size and ease of amplification from low quantity samples (Nilsson et al. 2008). Consequently, the use of ITS in yeast sequencing studies is increasing and ITS collections in online databases are expanding (Valente et al. 1999). A growing sequence database is advantageous since the more taxonomic coverage a database has, the greater the chance that any particular sequence obtained from the environment will yield a close database match (O'Brien et al. 2005).

ITS sequence data appear to be the default choice for species level fungal studies (Seifert 2009), but use of the ITS region also has limitations (Nilsson et al. 2008). The ITS region is variable in length, and although beneficial for providing superior resolution and identification in groups with longer amplicons, abundant insertion-deletions generate difficulties for alignment and phylogenetic construction (Seifert 2009). For example, ITS sequences from the genera Zygosaccharomyces and Torulaspora included extensive insertions and deletions, limiting the use of sequence alignment programs and assessment of more distant relationships (James et al. 1996). Establishment of a cut-off point or variation limit for species delimitation based on ITS sequences is also difficult due to the variable level of intraspecific ITS variation in fungi (O'Brien et al. 2005). ITS also has limitations for assessment of biodiversity; an investigation into the definition of species boundaries in tropical terrestrial endophytes found 
that sole use of the ITS region generally underestimated the predicted number of species, compared with estimates made using other nuclear loci (Gazis et al. 2011).

\subsubsection{Alternative Regions}

Molecular systematics and extensive databases have focused on the non-coding ITS region, however, coding regions such as the SSU and the D1/D2 variable domain of the LSU have also been used to analyse close and distant relationships (Kurtzman and Blanz 1998; Scorzetti et al. 2002). For example, the LSU enabled reliable identification of the majority of yeasts investigated due to its combination of conserved and variable regions (Fell and Kurtzman 1990; Kurtzman and Robnett 1998). One investigation examined yeasts from the Basidiomycota via sequence analysis of the ITS region and the D1/D2 region of the LSU; identification of most yeasts was possible with either sequence, but there were instances where ITS was able to separate closely related species when D1/D2 could not, and vice versa (Scorzetti et al. 2002). Although the LSU generally allows reliable identification of marine yeasts, analysis of additional regions may improve the confidence of identification procedures (Fell and Kurtzman 1990). The use of sequence data from multiple regions (including ITS and D1/D2) appears to be beneficial when comparing closely related sequences (James et al. 1996; Kurtzman and Fell 2006). Since phylogenetic analysis using a single gene can create uncertainty when assessing distant relationships, a multigene sequence approach has been used to analyse yeasts (Kurtzman and Robnett 2003); multigene analyses can be used to strengthen support for phylogenetic trees, which are often weak when constructed with single genes (Kurtzman and Fell 2006). Although they do not always enable separation of species, 
single gene sequences are still valuable for the rapid identification of some isolates via database searches (Kurtzman and Piškur 2006).

\subsection{Applications}

\subsubsection{Overview}

It has been suggested that marine fungi may have unusual physiological adaptations which have arisen from the distinct physio-chemical properties of marine environments (Raghukumar 2008). These adaptations have been exploited and developed for biotechnological uses (Raghukumar 2008), thus, marine yeasts have a wide range of current and potential applications (Chi et al. 2010). For example, the immunostimulatory abilities of marine yeasts have potential for use in aquaculture settings (Sajeevan et al. 2006; Reyesbecerril et al. 2008), and the biocontrol abilities of live marine yeasts can protect fruit against postharvest decay caused by pathogenic fungi (Wang et al. 2008). The biodegradative abilities of marine yeasts enable production of yeast biomass, which is of high nutritional quality and can be used in aquaculture or animal feed (Rhishipal and Philip 1998). Production of degradative extracellular enzymes is the main mode in which microorganisms take part in detrital processes, and can also be manipulated for biotechnological uses (Raghukumar 2008). Certain marine yeast species also produce various extracellular enzymes, including inulinase and killer toxin (Chi et al. 2009). Yeast byproducts or biomass can be produced efficiently due to the short generation time and limited requirements of yeasts (Kutty and Philip 2008, plus references therein). If marine yeasts are used instead of terrestrial yeasts for the 
production of byproducts or biomass, the freshwater required for these processes could be replaced with seawater, which is an abundant resource in many areas (Chi et al. 2006).

\subsubsection{Biomass}

Yeasts efficiently biodegrade organic matter or waste into yeast biomass (Rhishipal and Philip 1998). For example, certain marine yeasts isolated from the coast of India demonstrated efficient biotransformation of prawn shell waste into single cell protein; the biotransformation process conserved protein from the raw material and displayed additional protein enrichment (Rhishipal and Philip 1998). Marine yeasts have good amino acid composition plus high protein and carbohydrate levels, indicating potential for use as a dietary component in aquaculture (Brown et al. 1996). A marine strain of Yarrowia lipolytica (Wick., Kurtzman \& Herman) Van der Walt \& Arx contained more than $47 \mathrm{~g}$ of protein per $100 \mathrm{~g}$ of dry cell weight, and would be an excellent candidate for single cell protein production in the mariculture industry (Chi et al. 2007). During another investigation, two marine yeast species (Debaryomyces hansenii (Zopf) Lodder \& Kreger-van Rij and Candida austromarina (Fell \& I.L. Hunter) S.A. Mey. \& Yarrow, originally isolated from the coast of Korea) were fed to cladoceran Moina macrocopa (Straus), which has potential as a dietary component for fish larvae (Kang et al. 2006). In terms of nutritional quality, Candida austromarina was especially superior to the commercial diet Erythrobacter $s p$. S $\pi$-I used as a control (Kang et al. 2006). On a large scale, production and concentration of yeast cells is easy to manage, and certain species thrive on cheap agricultural byproducts (Chi et al. 2007). Overall, yeast single cell biomass is of high nutritional quality and would be a cost effective choice for 
supplementing animal or aquaculture feed, while utilizing a waste product (Rhishipal and Philip 1998).

\subsubsection{Immunostimulation and Biocontrol}

Bacteria and fungi have potential for use as prophylactic probiotics in aquaculture settings, where fish are susceptible to infections but antibiotic use is not appropriate for various reasons (Reyesbecerril et al. 2008). Yeasts are generally considered to be a commensal part of the gut flora in fish; live yeasts bind to the intestine and have potential probiotic activity (Gatesoupe 2007, plus references therein). When given as a dietary supplement, marine yeast Debaryomyces hansenii (originally isolated from the digestive system of rainbow trout) demonstrated adherence to the intestine and stimulation of some aspects of innate immunity in the seabream Sparus aurata L. (Reyesbecerril et al. 2008). Non-viable marine yeasts can also demonstrate immunostimulatory ability; one study investigated the effect of autoclaved marine yeast Candida sake (Saito \& Oda) Van Uden \& H.R. Buckley ex S.A. Mey. \& Ahearn (originally isolated from the coast of India) in the diet of shrimp Fenneropenaeus indicus (Milne-Edwards) (Sajeevan et al. 2006). Shrimps fed a diet with a Candida sake component of $10 \%$ exhibited a significant increase in immune response when challenged with white spot syndrome virus (Sajeevan et al. 2006). It was proposed that in penaeid shrimp, yeasts may simultaneously act as a nutritional supplement and an immunostimulant, and that the role of yeasts in immunostimulation is due to their nucleotides and cell wall glucan contents (Sajeevan et al. 2006). Data suggest that the $\beta$-glucan component of yeasts accounts for much of their immunostimulatory ability in fish, and that yeast extracts such as $\beta$-glucan can also be used as immunostimulants (Gatesoupe 2007, plus references therein). Penaeus monodon 
Fabricius demonstrated an enhanced survival rate and disease resistance when its diet was supplemented with $\beta$-1, 3-glucan from mushroom Schizophyllum commune Fr. (Chang 2000). If used for their immunostimulatory abilities, the halotolerant properties of marine yeasts would enable ease of application in brackish and seawater aquaculture systems (Sajeevan et al. 2006).

In addition to their immunoprotective abilities, marine yeasts have biocontrol potential against postharvest fungal disease (Wang et al. 2008). A Rhodosporidium paludigenum Fell \& Tallman isolate from the East China Sea was able to control black rot caused by Alternaria alternata (Fr.) Keissl. on cherry tomato fruit, possibly via competition for space and nutrition (Wang et al. 2008). The biocontrol ability of marine yeast species against postharvest grey mold caused by Botrytis cinerea Pers. on cherry tomatoes was also investigated; a washed cell suspension of Rhodosporidium paludigenum was the most efficacious treatment for decreasing disease incidence (Wang et al. 2010). An increase in Rhodosporidium paludigenum concentration was correlated with a decrease in disease incidence, suggesting that the mechanism of action was competition for nutrients, however, culture filtrate and autoclaved cultures also had some effect in vivo and may indicate further mechanisms (Wang et al. 2010). Rhodosporidium paludigenum is a suitable candidate for use in commercial processes due to its biocontrol abilities and resistance to osmotic stress, features that may be correlated with its isolation from the marine environment (Wang et al. 2010). Marine and terrestrial strains of Debaryomyces hansenii (originally isolated from Mexico) also demonstrated effective inhibition of postharvest blue mold caused by Penicillium italicum Wehmer on limes (Hernández-Montiel et al. 2010). These strains had an antagonistic nature, and competition for space and nutrients was suggested as a possible mechanism (HernándezMontiel et al. 2010). 


\subsubsection{Yeast Products}

Potential applications of marine yeasts include their utilization in the production of compounds such as pharmaceuticals, cosmetics, and chemicals for industry (Chi et al. 2006). Reviews describe a range of valuable products from marine yeasts, including bioemulsifiers (Nerurkar et al. 2009) and compounds such as vitamin c, glutathione and $\beta$-carotenoids (Chi et al. 2006). Marine yeasts from genera including Aureobasidium, Cryptococcus, Metschnikowia, Pichia and Yarrowia produce many extracellular enzymes: lipase, phytase, inulinase, alkaline protease, acid protease and killer toxin (Chi et al. 2009). Optimal conditions for the production of various enzymes were determined, and the presence of $\mathrm{Na}^{+}$ ions enhanced production in some cases (Chi et al. 2009). Certain enzymes have been purified and characterised, and have had their encoding genes cloned, sequenced and expressed (Chi et al. 2009). Certain enzymes have unique characteristics or encoding genes compared with homologous enzymes from their terrestrial counterparts (Chi et al. 2009). For example, marine yeast derived inulinase, lipase and phytase demonstrated higher activity than equivalent enzymes derived from terrestrial sources (Chi et al. 2009, plus references therein).

Inulinase is produced by many microorganisms, and is able to hydrolyse inulin to $95 \%$ pure fructose in one step (Gao et al. 2007). Inulinase can transform inulin (high levels of which are found in plants) into high fructose syrup, inulooligosaccharides and bioethanol (Chi et al. 2009). Microorganisms, particularly yeasts, are excellent producers of inulinases due to their high yields and ease of cultivation (Chi et al. 2009). Four marine yeasts (originally isolated from algae sampled in China, or sediment from the South China Sea) secreted large amounts of inulinase when grown on inulin containing media: Pichia guilliermondii Wick., Cryptococcus aureus (Saito) Takash., Sugita, Shinoda \& Nakase, Yarrowia lipolytica and 
Debaryomyces hansenii (Gao et al. 2007). Exoinulinase levels were highly variable between the yeasts isolated, as were the levels of monosaccharides, disaccharides and oligosaccharides produced by their crude inulinases (Gao et al. 2007). The $\mathrm{pH}$ and temperature required for optimal growth of these isolates was established, and although there was some variation between strains the ranges were in accordance with those from corresponding terrestrial yeasts (Gao et al. 2007). Nevertheless, some physical and biochemical differences have been observed between inulinases produced by marine and terrestrial yeasts (Chi et al. 2009).

Some marine yeasts produce killer toxin, which kills yeast of the same or related species but does not harm the killer strain itself (Magliani et al. 1997). Killer yeast strains are common and have been detected from a variety of genera: Debaryomyces, Candida, Pichia, Cryptococcus and Williopsis (Magliani et al. 1997). For example, marine yeast Pichia anomala (E.C. Hansen) Kurtzman (originally isolated from the gut of ascidians on the coast of China) produced killer toxin against Metschnikowia bicuspidata (Metschn.) T. Kamieński (Wang et al. 2007). Metschnikowia bicuspidata causes milky disease in the crab Portunus trituberculatus (Miers) and is responsible for great economic losses (Wang et al. 2007). Natural killer strains, such as Pichia anomala, have potential for controlling the growth of pathogenic yeasts including Metschnikowia bicuspidata in aquaculture settings, and may also be of use in other animals and plants (Wang et al. 2007). These unique properties are encouraging for further investigation of marine yeasts, which may be novel or enable production of compounds with novel characteristics (Chi et al. 2009). 


\subsubsection{Bioremediation}

Microorganisms take part in detrital processes via production of extracellular degradative enzymes (Raghukumar 2008). The degradation abilities of marine yeasts may enable bioremediation of contaminated marine environments by removing pollutants such as heavy metals and organic substances, especially hydrocarbons (Chi et al. 2010). For example, untreated palm oil mill effluent has a high chemical oxygen demand and is a source of pollution when released into rivers and lakes (Oswal et al. 2002). A marine strain of Yarrowia lipolytica (originally isolated from India) demonstrated degradation of raw palm oil mill effluent without dilution or nutrient addition, and reduced the chemical oxygen demand by around $95 \%$ (Oswal et al. 2002). This Yarrowia lipolytica strain was able to grow in saltwater or freshwater and over a wide range of $\mathrm{pH}$ levels, making it an excellent candidate for waste management (Oswal et al. 2002). Yarrowia lipolytica also enables the biotransformation of trinitrotoluene into less toxic products that can subsequently be utilized by other microorganisms (Jain et al. 2004), and has potential for the bioremediation of soil or water contaminated by heavy metals (Strouhal 2003). Marine yeasts may facilitate bioremediation via alternative mechanisms; a marine strain of Debaryomyces hansenii has potential as a sorbent for uranium removal due to its high sorption capacity, low biomass cost and ease of accessibility (Sarri et al. 2009).

1.5 New Zealand

Most investigations of New Zealand fungi have focused on the food and beverage industry, soils and pasture, plant pathogens and terrestrial ecosystems (Di Menna 1955; Fleet 2001; 
McKenzie 2001; Serjeant et al. 2008). Some information has been published regarding New Zealand's marine fungi (Lintott and Lintott 1982; Parsons and Fenwick 1984; Lintott and Lintott 2002), but no investigations are known to have surveyed marine yeast biodiversity from waters surrounding New Zealand's main Islands. Marine yeasts have been isolated from the Chatham Islands (Fell and Hunter 1968), which are geographically distant from the North and South Islands of New Zealand (Heath 1985). Twelve isolates of Candida natalensis Van der Walt \& Tscheuschner and a single isolate of Metschnikowia zobellii were collected from Chatham Island tidal pools, and six further isolates of Metschnikowia zobellii were found in a small freshwater lake (Fell and Hunter 1968). Isolates were identified based on their biochemical properties and mating characteristics with yeast type strains (Fell and Hunter 1968). The isolates from the freshwater ponds on Chatham Island were originally identified as Metschnikowia zobellii, but redesignated as Metschnikowia bicuspidata var. chathamia after further mating tests (Fell and Pitt 1969).

\subsection{Research Question}

There is a lack of information regarding the biodiversity of marine yeasts in New Zealand, yet they play an important ecological role and may have useful applications. The shortage of information has led to this investigation: the aim was to determine the marine yeast species present and their biodiversity in New Zealand. 


\section{Materials and Methods}

\subsection{Algal Collections}

Four algal collections were carried out in the Wellington Region (Fig. 1). Collection 1 was from Tarakena Bay on 18/12/09, Collections 2 and 3 were from Waitaha Cove on 29/01/10 and 25/02/10, and Collection 4 was from Onehunga Bay on 19/03/10. Sampling was carried out at low tide, and only completely submerged algae attached to the sea bed were collected. Where possible, two algal species were collected from each group: Rhodophyta, Chlorophyta and Phaeophyceae (Table 1). In Collection 1, five samples of each of the following algae were collected: Pachymenia lusoria (Greville) J. Agardh, Cladhymenia oblongifolia J.D. Hooker \& Harvey, Ulva sp. L., Macrocystis pyrifera and Carpophyllum maschalocarpum (Turner) Greville. In Collection 2, ten samples of each of the following algae were collected: Psaromenia berggernii (J. Agardh) D'Archino, W.A. Nelson \& Zuccarello, Champia novaezelandiae (J.D. Hooker \& Harvey) Harvey, Codium convolutum (Dellow) P.C. Silva, Caulerpa brownii (C. Agardh) Endlicher, Cystophora scalaris (J. Agardh) and Macrocystis pyrifera. In Collection 3, five samples were collected of the same algae that were sampled in Collection 2. In Collection 4, five samples were collected of the same algae that were sampled in Collections 2 and 3, with exception of the Rhodophyta. The same species belonging to the Rhodophyta could not be found in Collection 4 (except for an individual Psaromenia berggernii, which was sampled), so five samples from each of the species Cladhymenia oblongifolia and Pachymenia lusoria were collected. Each algal sample consisted of a $\sim 10 \mathrm{x}$ $10 \mathrm{~cm}$ sized piece taken from the blade (except for samples of Codium convolutum and Champia novae-zelandiae which consisted of a $\sim 3 \times 3 \times 3 \mathrm{~cm}$ sized algal mass). Each sample 
was collected in a sterile resealable plastic bag and given a code to reflect its origin, then transported to the lab on ice and processed on the same day.

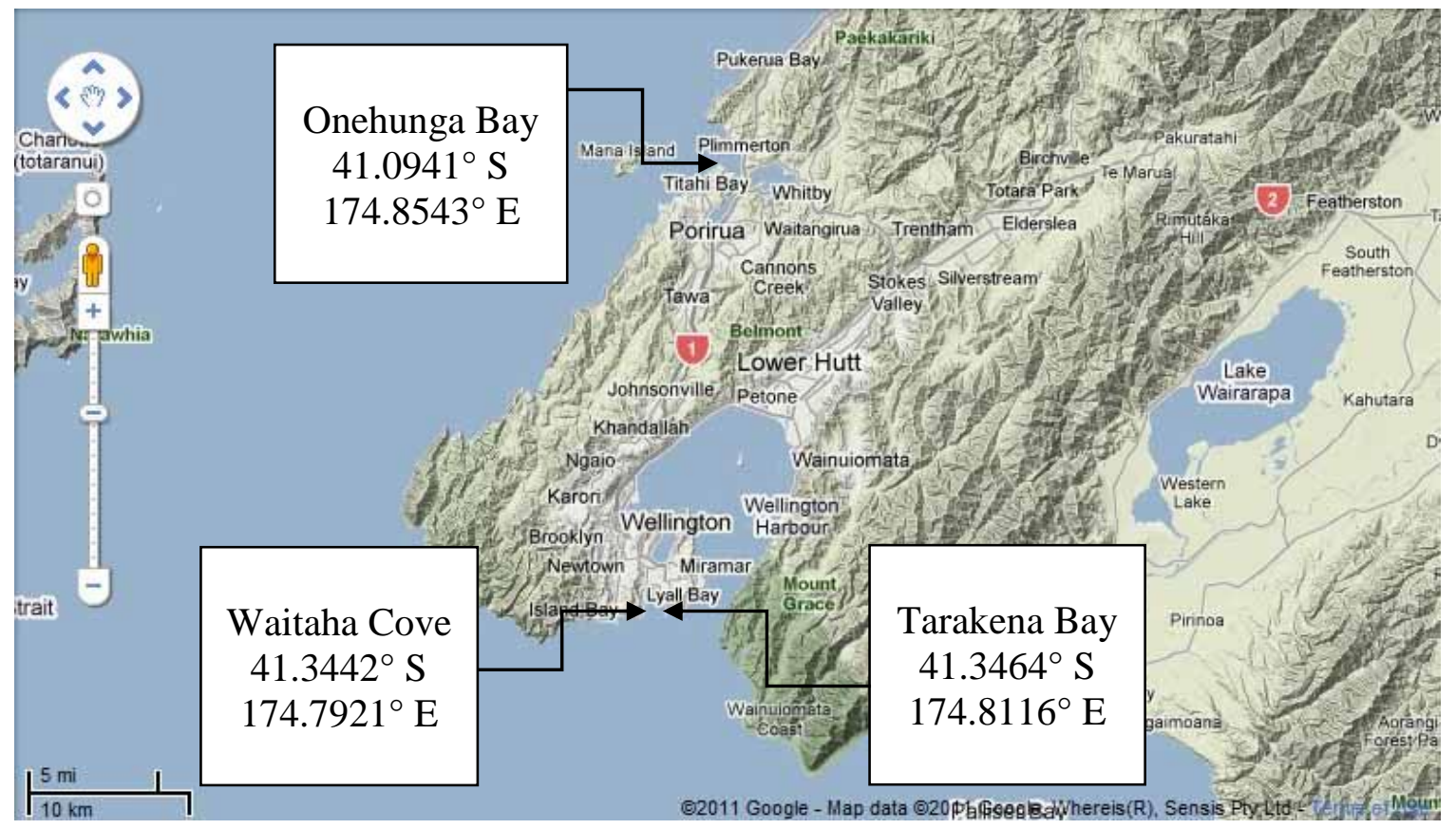

Figure 1. Map illustrating the location of algal collections conducted in the Wellington Region (Google Maps 2011).

\subsection{Initial Culture Techniques}

Based on positive growth results from trial investigations, two different nutrient media were chosen for marine yeast isolation (Appendix 5.2). These were Difco Standard Marine Agar Plus 1 (STD) and Difco Potato Dextrose Agar Plus (PDA), both with additional nutrients and PDA with additional salt (Appendix 5.1). Media was made up with the ingredients as specified, autoclaved and poured into Petri dishes one or two days prior to use. Sterile microbiological techniques were used at all times during preparation of the agar plates. 
To prevent bacterial overgrowth, filter-sterilised antibiotic solutions of streptomycin sulfate (in distilled water) and rifampicin (in methanol) were applied in a thin layer to each agar plate with a sterile disposable plastic spreader. During Collection 1, $100 \mu 1$ of streptomycin sulfate solution with concentration $10 \mathrm{mg} \mathrm{ml}^{-1}$ and $100 \mu \mathrm{l}$ of rifampicin solution with concentration 5 $\mathrm{mg} \mathrm{ml}^{-1}$ were applied to each plate. During Collections 2, 3 and 4, $100 \mu \mathrm{l}$ of streptomycin sulfate solution with concentration of $9 \mathrm{mg} \mathrm{ml}^{-1}$ and $100 \mu \mathrm{l}$ of rifampicin solution with concentration of $4.5 \mathrm{mg} \mathrm{ml}^{-1}$ were applied to each plate. The combination of streptomycin sulfate and rifampicin demonstrated effective inhibition of bacterial colony formation in trial investigations (Appendix 5.2). Antibiotics were applied to agar plates the day before collection, then covered with aluminium foil and left at room temperature overnight to prevent degradation of the antibiotics by light.

For Collection 1, three methods were used to inoculate agar plates with yeasts from the algae sampled. These were the sterile swabbing method (algal surface was rubbed with a sterile cotton swab which was transferred to an agar plate), the agitation of alga method (algal sample was shaken vigorously for 2 minutes in its original collection bag with $\sim 30 \mathrm{ml}$ of autoclaved sterile seawater, and a $100 \mu \mathrm{l}$ aliquot of the resultant solution was transferred to an agar plate) and the homogenisation of alga method (a $\sim 2 \times 2 \mathrm{~cm}$ algal sample was homogenised with a mini-blender in a falcon tube with $\sim 10 \mathrm{ml}$ of sterile seawater, and a 100 $\mu 1$ aliquot of the resultant solution was transferred to an agar plate). However, the homogenisation method was more time consuming than the other inoculation techniques and it was difficult to process the samples via all three methods within a single day. The homogenisation method was also thought to be the most prone to contamination, so it was not used for the remainder of the collections. For Collections 2, 3 and 4, only the sterile swabbing 
method and the agitation of alga method were used. Two agar plates were created for each transfer method per algal sample: one using STD and one using PDA. Thus, for each algal sample a total of four agar plates were created: a STD and a PDA plate using the sterile swabbing method, plus a STD and a PDA plate using the agitation of alga in sterile seawater method. A few plates were treated with an application of sterile seawater or rubbed with a sterile swab as a control. Agar plates were inverted and incubated at $18{ }^{\circ} \mathrm{C}$, then checked for colonies at one week and again at three weeks after inoculation.

\subsection{Isolate Processing}

In order to reduce the number of yeast isolates that were studied, individual colonies were selected for investigation. Selection was based on colony morphology; the criteria were colour, wet or dry surface, and colony elevation. One isolate from each morphological group was selected per algal sample, per collection. For example, if a plate had two pink colonies, two matt white colonies and two shiny white colonies, one colony from each group was selected. The isolates were given codes to reflect their alga of origin and which media they were isolated from: STD or PDA. Selected colonies were then subcultured onto fresh agar plates to eliminate possible fungal contamination (no antibiotics were required at this stage). Plates were incubated at $18{ }^{\circ} \mathrm{C}$ and checked after five days.

In order to ascertain that each yeast isolate was pure, a wet mount was made with a single colony taken from each subculture plate and viewed under an Olympus microscope. To establish purity, yeast isolates were required to have cells with a homogenous appearance and no observable contaminants, such as bacteria or filamentous fungi. Colony morphology and 
microscopic characteristics such as cell size and shape were observed and recorded. Photographs of agar plates were also taken with a Nikon D90 SLR camera, using automatic settings for conditions with fluorescent white light. If there were any doubts about the purity of an isolate it was subcultured again until pure. When the purity of an isolate was established, a liquid culture was made by inoculating a sample of the individual colony into 1 $\mathrm{ml}$ of Difco Marine Broth Plus and incubating for five days at $18^{\circ} \mathrm{C}$. During later collections, Difco Luria Broth was used as it was an equally effective liquid media for yeast growth and contained less sediment which was beneficial for microscopy. After incubation, a sample of broth from each isolate was checked via microscopy to ensure that the liquid culture was pure. A Leica DM IL inverted microscope was used and images were taken with an attached Olympus C5060WZ camera. A $1.5 \mathrm{ml}$ aliquot of broth from each isolate was taken for DNA extraction. An additional $600 \mu \mathrm{l}$ aliquot of broth was taken from each isolate, mixed with 550 $\mu 1$ of an autoclaved glycerol solution (45\% glycerol in aged seawater) and preserved at -80 ${ }^{\circ} \mathrm{C}$. This method was tested and found to ensure the survival of yeast isolates; frozen samples were placed on ice while yeasts were resuscitated onto fresh agar plates.

\subsection{DNA Extraction}

A $1.5 \mathrm{ml}$ aliquot of each yeast liquid culture was spun down for 2 minutes at $12,000 \mathrm{G}$ in a $1.5 \mathrm{ml}$ Eppendorf tube to produce a pellet. The supernatant was discarded and DNA extraction was carried out. $500 \mu \mathrm{l}$ of CTAB extraction buffer (2\% CTAB, $0.1 \mathrm{M}$ Tris-HCl (pH 8.0), $1.4 \mathrm{M} \mathrm{NaCl}, 20 \mathrm{mM}$ EDTA, $1 \% \mathrm{PVP})$ plus $2 \mu \mathrm{l}$ of RNase A (100 $\mathrm{mg} \mathrm{ml}^{-1}$ ) and $5 \mu 1$ of Proteinase K (20 mg ml$\left.{ }^{-1}\right)$ were added to each pellet. Samples were incubated at $\sim 57{ }^{\circ} \mathrm{C}$ overnight, with occasional mixing. An equal volume of chloroform:isoamyl alcohol (24:1) 
was added to each sample. Samples were gently mixed on a rocking table for 2 minutes and spun at $12,000 \mathrm{G}$ for 10 minutes. The supernatant (aqueous phase) was extracted and reserved in a new tube. An equal volume of chloroform:isoamyl alcohol (24:1) was added to the samples, which were gently rocked for 2 minutes then spun at $12,000 \mathrm{G}$ for 5 minutes. The aqueous phase was retained in a new tube, to which an equal volume of $100 \%$ isopropanol was added. Samples were inverted a few times, then placed at room temperature for 30 minutes and spun at $12,000 \mathrm{G}$ for 20 minutes. The liquid phase was decanted and $500 \mu \mathrm{l}$ of 70 $\%$ ethanol was added to each pellet. Samples were inverted a few times and spun at $12,000 \mathrm{G}$ for 5 minutes. The liquid phase was decanted and the pellet allowed to air dry before $50 \mu$ of a $0.1 \mathrm{X}$ TE (Tris/EDTA) buffer was added. Samples were stored at $-20{ }^{\circ} \mathrm{C}$ until required.

\subsection{PCR and Sequencing}

For each isolate, the ITS rRNA gene region was amplified from an aliquot of the DNA extraction samples using ITS1 (TCCGTAGGTGAACCTGCGG) and ITS4 (TCCTCCGCTTATTGATATGC) primers (White et al. 1990). The reaction mixture had a total volume of $30 \mu \mathrm{l}$, and was comprised of a 1X New England BioLabs Buffer (including 2 $\mathrm{mM} \mathrm{MgCl}_{2}$ ), $208 \mu \mathrm{M}$ dNTPs, $0.00833 \%$ bovine serum albumin, 250 pM ITS1, 250 pM ITS4, and included 1U New England BioLabs Taq polymerase. $1 \mu 1$ of DNA extraction product was used for each PCR reaction and is included in the total volume of $30 \mu \mathrm{l}$. A touchdown PCR was carried out and the amplification conditions were as follows: preliminary denaturation at $94{ }^{\circ} \mathrm{C}$ for 5 minutes, followed by five cycles of denaturation at $94{ }^{\circ} \mathrm{C}$ for 1 minute, annealing at $55^{\circ} \mathrm{C}$ for 45 seconds and extension at $72{ }^{\circ} \mathrm{C}$ for 1 minute. The annealing temperature was decreased by $1{ }^{\circ} \mathrm{C}$ every cycle. This was followed by a further 28 cycles with denaturation at 
$94{ }^{\circ} \mathrm{C}$ for 1 minute, annealing at $50{ }^{\circ} \mathrm{C}$ for 45 seconds and extension at $72{ }^{\circ} \mathrm{C}$ for 1 minute. Final extension was at $72{ }^{\circ} \mathrm{C}$ for 5 minutes. An aliquot of each PCR product $(4 \mu \mathrm{l})$ was electrophoresed on a $1 \%$ agarose gel made with $0.5 \mathrm{X}$ TBE (Tris/Borate/EDTA) in a buffer of 0.5X TBE. The gel was stained in an ethidium bromide solution for 15 minutes and viewed under ultraviolet light to ensure that the PCR products were of the expected size and that the DNA was of sufficient quantity for sequencing. The remainder of the successful PCR products (volume $\sim 26 \mu \mathrm{l}$ ) were purified by adding $0.5 \mu \mathrm{l}$ of ExoSAP-IT to each sample and incubating as per manufacturer's instructions $\left(15\right.$ minutes at $37{ }^{\circ} \mathrm{C}$ followed by 15 minutes at $80{ }^{\circ} \mathrm{C}$ for inactivation (Affymetrix)). PCR products were sequenced commercially by Macrogen Inc.

\subsection{Data Analysis}

ITS sequences from yeast isolates were imported into Geneious version 5.4.5 (Drummond et al. 2011). The ITS1 and ITS4 sequence for each isolate were assembled into a contig (a consensus sequence created with overlapping DNA segments) using default settings. For isolates that did not form a contig or formed one with little overlap, the sequence with the best quality and length was chosen for further analysis (this was always the sequence from the ITS4 primer side). The few ITS sequences that were very short or had poor quality were removed from this investigation. ITS sequences were manually checked by eye for errors, and any ambiguities were replaced with the correct nucleotide if it was possible to do so with confidence. The ends of the ITS sequences were manually trimmed to remove primers and regions of poor quality sequence as required; most sequences had poor quality ends that did not contain the full primer sequence so required removal of primer fragments. The lengths of 
ITS sequences that formed contigs (after removal of primers and poor quality sequences) were generally in agreement with corresponding PCR product lengths from agarose gel electrophoresis and expected ITS lengths from the literature (CBS-KNAW 2012; MycoBank 2012). ITS sequences were exported in FASTA format in order to carry out database searches.

Each edited ITS sequence was queried against the National Center for Biotechnology Information sequence database: GenBank (Benson et al. 2005). The Basic Local Alignment Search Tool (BLAST) was used, with selection of the Nucleotide collection (nr/nt) and Megablast (optimised for highly similar sequences) (Altschul et al. 1990). For each sequence queried, the BLAST result with the highest identity plus four additional high identity sequences (from different sources if possible) were selected and recorded from GenBank in order of their appearance (data not shown). Parameters such as E-values were also recorded. Selected ITS sequences from GenBank and the literature were imported into Geneious for further analysis, and divided into groups representing four subphyla: Pezizomycotina, Saccharomycotina (both from the Ascomycota), Agaricomycotina and Pucciniomycotina (both from the Basidiomycota). Yeasts isolated during this investigation were assigned to the subphylum they shared the highest ITS sequence similarity with. ITS sequence alignments were created for each subphylum using the standard Geneious alignment procedure (Drummond et al. 2011). The option of global alignment with free end gaps and associated default settings were used. Alignments were checked and edited for accuracy manually and the ends trimmed where appropriate. Neighbour-joining phylogenetic trees were constructed using the Tamura-Nei genetic distance model (Tamura and Nei 1993). Bootstrap values were obtained via resampling with default bootstrap settings and 100 replicates. 


\section{Results}

\subsection{Collection Data}

\subsubsection{Algal Collections}

Four algal collections were carried out during this investigation (Table 1). Collection 1 was from Tarakena Bay on 18/12/09 where the sea surface temperature was $14.8^{\circ} \mathrm{C}$. Collections 2 and 3 were from Waitaha Cove. Collection 2 was on 29/01/10 where the sea surface temperature was $14.0{ }^{\circ} \mathrm{C}$ and Collection 3 was on $25 / 02 / 10$ where the sea surface temperature was $17.9{ }^{\circ} \mathrm{C}$. Collection 4 was from Onehunga Bay on 19/03/10 where the sea surface temperature was $16.2{ }^{\circ} \mathrm{C}$. A range of different algal species from Rhodophyta, Chlorophyta and Phaeophyceae were sampled during these collections. The algal species sampled and the total number of yeasts isolated from each alga was variable between collections.

\subsubsection{Inoculation Plates}

Marine yeasts were isolated from algal samples as described above. After inoculation from algae and one week of incubation at $18{ }^{\circ} \mathrm{C}$, agar plates displayed an assortment of yeasts and filamentous fungal colonies (Fig. 2). Yeast colonies were selected for isolation and subcultured as described above. The control plate, which was treated with a sterile swab and sterile seawater, yielded no visible colonies. 
Table 1. Particulars regarding algae sampled in the Wellington Region.

\begin{tabular}{|c|c|c|c|c|}
\hline Algal Code & Algal Group & Algal Species & $\begin{array}{c}\text { Number of } \\
\text { Algal } \\
\text { Samples } \\
\text { Collected }\end{array}$ & $\begin{array}{c}\text { Number of } \\
\text { Individual } \\
\text { Yeasts } \\
\text { Isolated }\end{array}$ \\
\hline \multicolumn{5}{|c|}{ Collection 1: $18 / 12 / 09$ from Tarakena Bay $\left(14.8^{\circ} \mathrm{C}\right)$} \\
\hline $\mathrm{B}$ & \multirow[b]{2}{*}{ Rhodophyta } & Pachymenia lusoria & 5 & 1 \\
\hline $\mathrm{C}$ & & $\begin{array}{l}\text { Cladhymenia } \\
\text { oblongifolia }\end{array}$ & 5 & 1 \\
\hline $\mathrm{D}$ & Chlorophyta & Ulva sp. & 5 & 1 \\
\hline $\mathrm{E}$ & \multirow[b]{2}{*}{ Phaeophyceae } & Macrocystis pyrifera & 5 & 3 \\
\hline $\mathrm{F}$ & & $\begin{array}{l}\text { Carpophyllum } \\
\text { maschalocarpum }\end{array}$ & 5 & 0 \\
\hline \multicolumn{5}{|c|}{ Collection 2: 29/01/10 from Waitaha Cove $\left(14.0^{\circ} \mathrm{C}\right)$} \\
\hline $\mathrm{H}$ & \multirow[b]{2}{*}{ Rhodophyta } & Psaromenia berggernii & 10 & 4 \\
\hline I & & $\begin{array}{l}\text { Champia } \\
\text { novae-zelandiae }\end{array}$ & 10 & 6 \\
\hline $\mathrm{J}$ & \multirow[b]{2}{*}{ Chlorophyta } & Codium convolutum & 10 & 5 \\
\hline KK & & Caulerpa brownii & 10 & 3 \\
\hline $\mathrm{L}$ & \multirow[b]{2}{*}{ Phaeophyceae } & Cystophora scalaris & 10 & 7 \\
\hline $\mathrm{M}$ & & Macrocystis pyrifera & 10 & 2 \\
\hline \multicolumn{5}{|c|}{ Collection 3: $25 / 02 / 10$ from Waitaha Cove $\left(17.9^{\circ} \mathrm{C}\right)$} \\
\hline $\mathrm{N}$ & \multirow[b]{2}{*}{ Chlorophyta } & Caulerpa brownii & 5 & 8 \\
\hline $\mathrm{O}$ & & Codium convolutum & 5 & 8 \\
\hline $\mathrm{P}$ & \multirow[b]{2}{*}{ Phaeophyceae } & Macrocystis pyrifera & 5 & 7 \\
\hline $\mathrm{Q}$ & & Cystophora scalaris & 5 & 7 \\
\hline $\mathrm{R}$ & \multirow[b]{2}{*}{ Rhodophyta } & $\begin{array}{l}\text { Champia } \\
\text { novae-zelandiae }\end{array}$ & 5 & 6 \\
\hline $\mathrm{S}$ & & Psaromenia berggernii & 5 & 6 \\
\hline \multicolumn{5}{|c|}{ Collection 4: 19/03/10 from Onehunga Bay $\left(16.2^{\circ} \mathrm{C}\right)$} \\
\hline $\mathrm{T}$ & \multirow[b]{2}{*}{ Phaeophyceae } & Macrocystis pyrifera & 5 & 3 \\
\hline $\mathrm{U}$ & & Cystophora scalaris & 5 & 4 \\
\hline $\mathrm{V}$ & \multirow[b]{3}{*}{ Rhodophyta } & $\begin{array}{l}\text { Cladhymenia } \\
\text { oblongifolia }\end{array}$ & 5 & 4 \\
\hline $\mathrm{W}$ & & Pachymenia lusoria & 5 & 1 \\
\hline $\mathrm{X} 2$ & & Psaromenia berggernii & 1 & 1 \\
\hline $\mathrm{Y}$ & \multirow[b]{2}{*}{ Chlorophyta } & Codium convolutum & 5 & 3 \\
\hline $\mathrm{Z}$ & & Caulerpa brownii & 5 & 3 \\
\hline
\end{tabular}



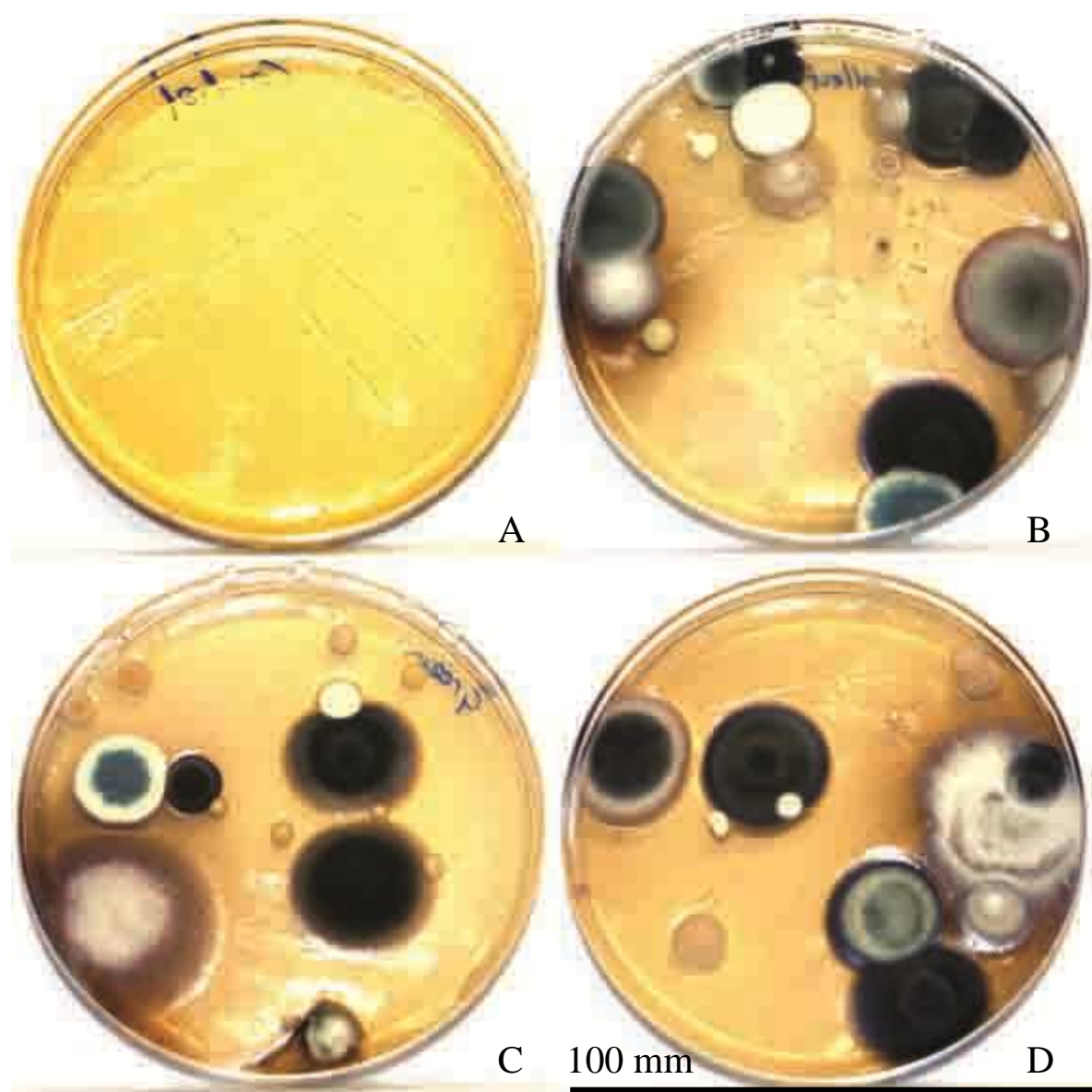

Figure 2. Photographs illustrating the appearance of agar plates, after inoculation with yeasts from algae and one week of incubation at $18{ }^{\circ} \mathrm{C}$. These agar plates have been created to reflect the appearance of original isolation plates, which were not photographed. The recreated agar plates were inoculated with yeasts from a fresh algal sample using the same isolation techniques, media, antibiotics and incubation conditions employed during this investigation. Plate A was inoculated with a sterile swab and sterile seawater only, as a control. Plates B, C and D display small cream coloured yeast colonies, and large filamentous fungal colonies coloured black, white and green. 


\subsubsection{Yeast Isolation}

During this investigation, 106 individual yeasts were selected for isolation from original algal-inoculated agar plates. Ninety-four of these yeasts were successfully isolated and found to be pure via the methods described above. ITS PCR products from 86 yeast isolates were sent away for sequencing.

3.2 Isolate Identification

\subsubsection{ITS Sequences}

ITS PCR products from 86 of 94 successfully isolated yeasts were sent away for sequencing. ITS sequences from 12 of these isolates were omitted due to poor quality sequence data. The remaining 74 isolates yielded ITS sequences of sufficient quality for yeast identification to at least the genus level and sometimes to the species level (Table 2). Based on their similarity with ITS sequences obtained from GenBank, good quality ITS sequences could be confidently separated into four subphyla: Pezizomycotina, Saccharomycotina, Agaricomycotina and Pucciniomycotina. ITS sequences from three isolates in the subphylum Pezizomycotina were obtained, all of which were of good quality and formed contigs ranging from 526 to $541 \mathrm{bp}$ in length. ITS sequences from 50 isolates in the subphylum Saccharomycotina were obtained, but only one contig was generated (383 bp). ITS sequences from the remaining 49 Saccharomycotina isolates did not form contigs; since they were of better quality than sequences from the ITS1 primer side, partial sequences from the ITS4 
primer side (159 to $247 \mathrm{bp}$ ) were used during further analysis. ITS sequences from 12 isolates in the subphylum Agaricomycotina were obtained, all of which were of good quality and formed contigs ranging from 421 to $603 \mathrm{bp}$ in length. Similarly, ITS sequences from nine isolates in the subphylum Pucciniomycotina were obtained, all of which were of good quality and formed contigs ranging from 539 to $599 \mathrm{bp}$ in length.

\subsubsection{GenBank}

A BLAST search queried good quality ITS sequences from 74 isolates against the sequence database GenBank, and most sequences yielded BLAST results with high sequence identity (97- $100 \%$ ) (Table 2). For some ITS sequences, the BLAST result with the highest sequence identity was from a named species, allowing confident identification of the corresponding isolate based on GenBank search results alone. However, further information was required as GenBank results did not enable confident identification of all isolates.

\subsubsection{Phylogenetic Analysis}

Phylogenetic analysis was carried out using ITS sequences from yeasts isolated during this investigation and selected ITS sequences from GenBank search results. For each isolate with good quality ITS sequence data, a comparison was made between GenBank search results and the closest neighbouring organism from phylogenetic trees (Table 2). For isolates from the Basidiomycota, phylogenetic trees demonstrated good species separation; phylogenetic trees containing Agaricomycotina or Pucciniomycotina isolates were generally in agreement with 
results from GenBank searches, providing confident isolate identification. Most isolates from the Ascomycota were in the subphylum Saccharomycotina and did not demonstrate good species separation in phylogenetic trees; phylogenetic trees could not confirm GenBank search results or identify many Saccharomycotina isolates further than the genus level.

\subsubsection{Culture Studies}

Although sequence data were the main source of information for isolate identification, observations made during culture studies were useful for confirmation of these results. A range of features including colony colour and surface appearance, plus microscopic size and shape were recorded. Observations made during this investigation were compared to descriptions of yeast species from the literature, particularly from fungal databases MycoBank (MycoBank 2012) and Centraalbureau voor Schimmelcultures (CBS-KNAW 2012). For most isolates, features observed during this investigation and descriptions from the literature were in agreement with initial isolate identifications made based on ITS sequence data. 
Table 2. Isolate identification based on GenBank search results and phylogenetic analysis using ITS sequence data.

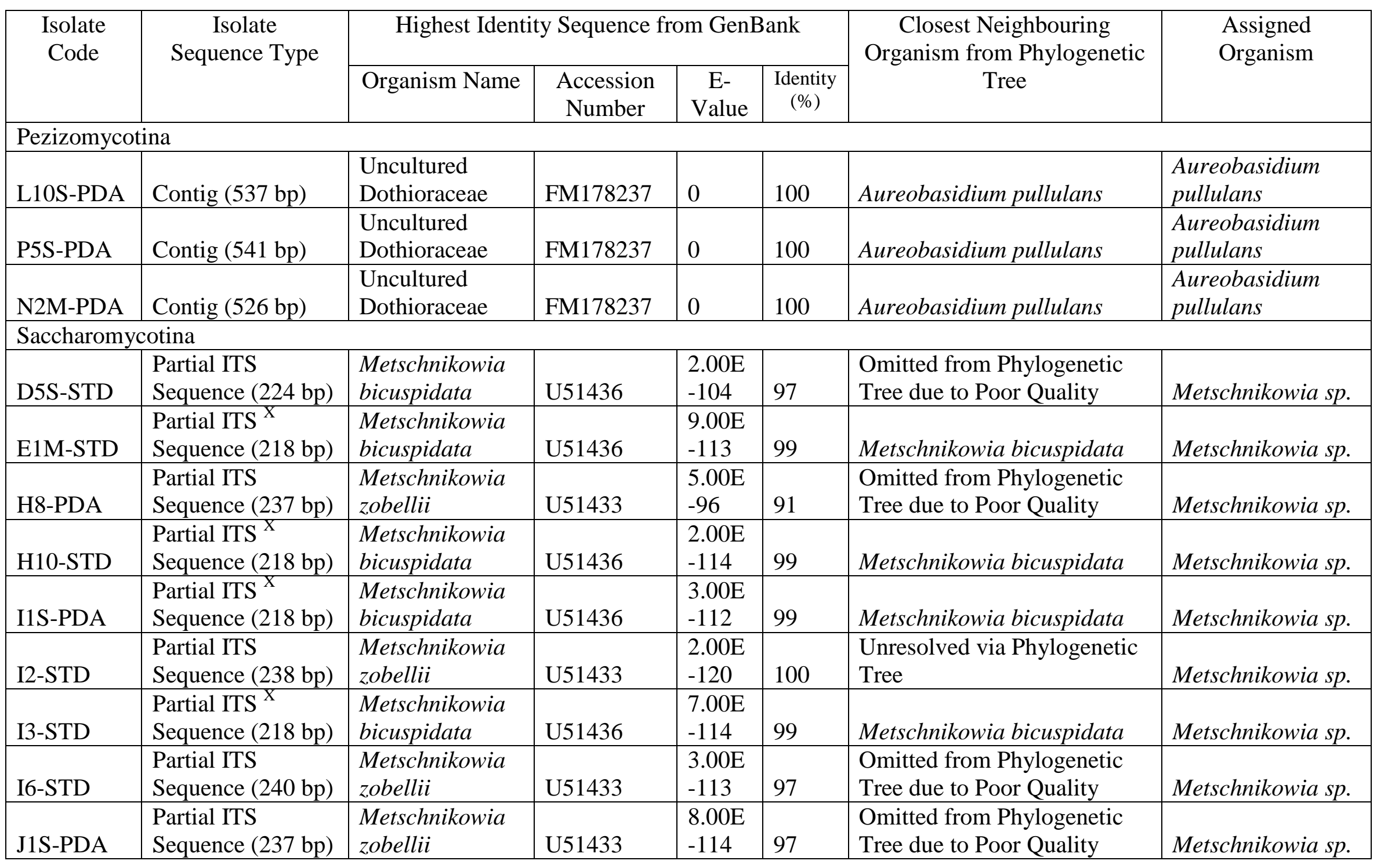




\begin{tabular}{|c|c|c|c|c|c|c|c|}
\hline J3S-PDA & $\begin{array}{l}\text { Partial ITS } X \\
\text { Sequence }(218 \mathrm{bp})\end{array}$ & $\begin{array}{l}\text { Metschnikowia } \\
\text { bicuspidata }\end{array}$ & U51436 & $\begin{array}{l}1.00 \mathrm{E} \\
-116\end{array}$ & 99 & Metschnikowia bicuspidata & Metschnikowia sp. \\
\hline J5S-PDA & $\begin{array}{l}\text { Partial ITS } \\
\text { Sequence (165 bp) }\end{array}$ & $\begin{array}{l}\text { Metschnikowia } \\
\text { reukaufii }\end{array}$ & HQ221884 & $\begin{array}{l}5.00 \mathrm{E} \\
-79\end{array}$ & 99 & $\begin{array}{l}\text { Omitted from Phylogenetic } \\
\text { Tree due to Poor Quality }\end{array}$ & Metschnikowia sp. \\
\hline J8S-STD & $\begin{array}{l}\text { Partial ITS } \\
\text { Sequence (238 bp) }\end{array}$ & $\begin{array}{l}\text { Metschnikowia } \\
\text { zobellii }\end{array}$ & U51433 & $\begin{array}{l}6.00 \mathrm{E} \\
-100\end{array}$ & 93 & $\begin{array}{l}\text { Omitted from Phylogenetic } \\
\text { Tree due to Poor Quality }\end{array}$ & Metschnikowia sp. \\
\hline $\begin{array}{l}\text { KK5S- } \\
\text { STD }\end{array}$ & $\begin{array}{l}\text { Partial ITS } \\
\text { Sequence (215 bp) }\end{array}$ & $\begin{array}{l}\text { Metschnikowia } \\
\text { bicuspidata }\end{array}$ & U51436 & $\begin{array}{l}5.00 \mathrm{E} \\
-105\end{array}$ & 100 & $\begin{array}{l}\text { Metschnikowia bicuspidata } \\
\text { (Possibly var. bicuspidata or } \\
\text { var. chathamia) }\end{array}$ & Metschnikowia sp. \\
\hline $\begin{array}{l}\text { KK8S- } \\
\text { STD }\end{array}$ & $\begin{array}{l}\text { Partial ITS } X \\
\text { Sequence }(218 \mathrm{bp})\end{array}$ & $\begin{array}{l}\text { Metschnikowia } \\
\text { bicuspidata }\end{array}$ & U51436 & $\begin{array}{l}3.00 \mathrm{E} \\
-112 \\
\end{array}$ & 99 & Metschnikowia bicuspidata & Metschnikowia sp. \\
\hline $\begin{array}{l}\text { L2S-2- } \\
\text { PDA }\end{array}$ & $\begin{array}{l}\text { Partial ITS } \\
\text { Sequence }(185 \mathrm{bp})\end{array}$ & $\begin{array}{l}\text { Metschnikowia } \\
\text { bicuspidata }\end{array}$ & EF643581 & $\begin{array}{l}8.00 \mathrm{E} \\
-88\end{array}$ & 98 & $\begin{array}{l}\text { Omitted from Phylogenetic } \\
\text { Tree due to Poor Quality }\end{array}$ & Metschnikowia sp. \\
\hline L10S-STD & $\begin{array}{l}\text { Partial ITS } \mathrm{X} \\
\text { Sequence }(218 \mathrm{bp})\end{array}$ & $\begin{array}{l}\text { Metschnikowia } \\
\text { bicuspidata }\end{array}$ & U51436 & $\begin{array}{l}3.00 \mathrm{E} \\
-113\end{array}$ & 99 & Metschnikowia bicuspidata & Metschnikowia sp. \\
\hline M7-STD & $\begin{array}{l}\text { Partial ITS } \\
\text { Sequence }(230 \mathrm{bp})\end{array}$ & $\begin{array}{l}\text { Metschnikowia } \\
\text { bicuspidata }\end{array}$ & U51436 & $\begin{array}{l}3.00 \mathrm{E} \\
-108\end{array}$ & 98 & $\begin{array}{l}\text { Omitted from Phylogenetic } \\
\text { Tree due to Poor Quality }\end{array}$ & Metschnikowia sp. \\
\hline N1M-PDA & $\begin{array}{l}\text { Partial ITS } \\
\text { Sequence }(211 \mathrm{bp})\end{array}$ & $\begin{array}{l}\text { Metschnikowia } \\
\text { bicuspidata }\end{array}$ & EF643581 & $\begin{array}{l}3.00 \mathrm{E} \\
-102\end{array}$ & 99 & $\begin{array}{l}\text { Omitted from Phylogenetic } \\
\text { Tree due to Poor Quality }\end{array}$ & Metschnikowia sp. \\
\hline N2M-STD & $\begin{array}{l}\text { Partial ITS } X \\
\text { Sequence }(218 \mathrm{bp})\end{array}$ & $\begin{array}{l}\text { Metschnikowia } \\
\text { bicuspidata }\end{array}$ & U51436 & $\begin{array}{l}2.00 \mathrm{E} \\
-103 \\
\end{array}$ & 100 & Metschnikowia bicuspidata & Metschnikowia sp. \\
\hline N4M-STD & $\begin{array}{l}\text { Partial ITS } \\
\text { Sequence (159 bp) }\end{array}$ & $\begin{array}{l}\text { Metschnikowia } \\
\text { reukaufii }\end{array}$ & HQ221884 & $\begin{array}{l}5.00 \mathrm{E} \\
-74\end{array}$ & 98 & $\begin{array}{l}\text { Omitted from Phylogenetic } \\
\text { Tree due to Poor Quality }\end{array}$ & Metschnikowia sp. \\
\hline N5M-PDA & $\begin{array}{l}\text { Partial ITS } \\
\text { Sequence (229 bp) }\end{array}$ & $\begin{array}{l}\text { Metschnikowia } \\
\text { bicuspidata }\end{array}$ & EF643581 & $\begin{array}{l}6.00 \mathrm{E} \\
-110 \\
\end{array}$ & 97 & $\begin{array}{l}\text { Omitted from Phylogenetic } \\
\text { Tree due to Poor Quality }\end{array}$ & Metschnikowia sp. \\
\hline N5M-STD & $\begin{array}{l}\text { Partial ITS } \\
\text { Sequence ( } 245 \mathrm{bp})\end{array}$ & $\begin{array}{l}\text { Metschnikowia } \\
\text { zobellii }\end{array}$ & U51433 & $\begin{array}{l}1.00 \mathrm{E} \\
-112 \\
\end{array}$ & 99 & Metschnikowia zobellii & $\begin{array}{l}\text { Metschnikowia } \\
\text { zobellii }\end{array}$ \\
\hline O1M-PDA & $\begin{array}{l}\text { Partial ITS }{ }^{X} \\
\text { Sequence }(218 \mathrm{bp})\end{array}$ & $\begin{array}{l}\text { Metschnikowia } \\
\text { bicuspidata }\end{array}$ & U51436 & $\begin{array}{l}2.00 \mathrm{E} \\
-114\end{array}$ & 99 & Metschnikowia bicuspidata & Metschnikowia sp. \\
\hline O1M-STD & $\begin{array}{l}\text { Partial ITS } \\
\text { Sequence }(210 \mathrm{bp})\end{array}$ & $\begin{array}{l}\text { Metschnikowia } \\
\text { bicuspidata }\end{array}$ & U51436 & $\begin{array}{l}9.00 \mathrm{E} \\
-103\end{array}$ & 99 & $\begin{array}{l}\text { Omitted from Phylogenetic } \\
\text { Tree due to Poor Quality }\end{array}$ & Metschnikowia sp. \\
\hline
\end{tabular}




\begin{tabular}{|c|c|c|c|c|c|c|c|}
\hline O3M-PDA & $\begin{array}{l}\text { Partial ITS } X \\
\text { Sequence }(218 \mathrm{bp})\end{array}$ & $\begin{array}{l}\text { Metschnikowia } \\
\text { bicuspidata }\end{array}$ & U51436 & $\begin{array}{l}3.00 \mathrm{E} \\
-113\end{array}$ & 99 & Metschnikowia bicuspidata & Metschnikowia sp. \\
\hline O5M-PDA & $\begin{array}{l}\text { Partial ITS } \mathrm{X} \\
\text { Sequence }(218 \mathrm{bp})\end{array}$ & $\begin{array}{l}\text { Metschnikowia } \\
\text { bicuspidata }\end{array}$ & U51436 & $\begin{array}{l}7.00 \mathrm{E} \\
-114\end{array}$ & 99 & Metschnikowia bicuspidata & Metschnikowia sp. \\
\hline O5M-STD & $\begin{array}{l}\text { Partial ITS } \\
\text { Sequence (244 bp) }\end{array}$ & $\begin{array}{l}\text { Metschnikowia } \\
\text { zobellii }\end{array}$ & U51433 & $\begin{array}{l}6.00 \mathrm{E} \\
-95\end{array}$ & 89 & $\begin{array}{l}\text { Omitted from Phylogenetic } \\
\text { Tree due to Poor Quality }\end{array}$ & Metschnikowia sp. \\
\hline O5S-PDA & $\begin{array}{l}\text { Partial ITS } \\
\text { Sequence (210 bp) }\end{array}$ & $\begin{array}{l}\text { Metschnikowia } \\
\text { bicuspidata }\end{array}$ & U51436 & $\begin{array}{l}1.00 \mathrm{E} \\
-101 \\
\end{array}$ & 99 & $\begin{array}{l}\text { Omitted from Phylogenetic } \\
\text { Tree due to Poor Quality }\end{array}$ & Metschnikowia sp. \\
\hline O5S-STD & $\begin{array}{l}\text { Partial ITS } X \\
\text { Sequence }(218 \mathrm{bp})\end{array}$ & $\begin{array}{l}\text { Metschnikowia } \\
\text { bicuspidata }\end{array}$ & U51436 & $\begin{array}{l}3.00 \mathrm{E} \\
-112 \\
\end{array}$ & 99 & Metschnikowia bicuspidata & Metschnikowia sp. \\
\hline $\begin{array}{l}\text { P1M-2- } \\
\text { PDA }\end{array}$ & $\begin{array}{l}\text { Partial ITS } \mathrm{X} \\
\text { Sequence }(218 \mathrm{bp})\end{array}$ & $\begin{array}{l}\text { Metschnikowia } \\
\text { bicuspidata }\end{array}$ & U51436 & $\begin{array}{l}2.00 \mathrm{E} \\
-114\end{array}$ & 99 & Metschnikowia bicuspidata & Metschnikowia sp. \\
\hline P3S-STD & $\begin{array}{l}\text { Partial ITS }{ }^{X} \\
\text { Sequence }(218 \mathrm{bp})\end{array}$ & $\begin{array}{l}\text { Metschnikowia } \\
\text { bicuspidata }\end{array}$ & U51436 & $\begin{array}{l}3.00 \mathrm{E} \\
-112 \\
\end{array}$ & 99 & Metschnikowia bicuspidata & Metschnikowia sp. \\
\hline Q1M-PDA & $\begin{array}{l}\text { Partial ITS } \mathrm{X} \\
\text { Sequence }(218 \mathrm{bp})\end{array}$ & $\begin{array}{l}\text { Metschnikowia } \\
\text { bicuspidata }\end{array}$ & U51436 & $\begin{array}{l}2.00 \mathrm{E} \\
-104\end{array}$ & 100 & Metschnikowia bicuspidata & Metschnikowia sp. \\
\hline Q1M-STD & $\begin{array}{l}\text { Partial ITS } \mathrm{X} \\
\text { Sequence }(218 \mathrm{bp})\end{array}$ & $\begin{array}{l}\text { Metschnikowia } \\
\text { bicuspidata }\end{array}$ & U51436 & $\begin{array}{l}3.00 \mathrm{E} \\
-113\end{array}$ & 99 & Metschnikowia bicuspidata & Metschnikowia sp. \\
\hline Q2M-STD & $\begin{array}{l}\text { Partial ITS } X \\
\text { Sequence }(218 \mathrm{bp})\end{array}$ & $\begin{array}{l}\text { Metschnikowia } \\
\text { bicuspidata }\end{array}$ & U51436 & $\begin{array}{l}7.00 \mathrm{E} \\
-114\end{array}$ & 99 & Metschnikowia bicuspidata & Metschnikowia sp. \\
\hline Q4S-STD & $\begin{array}{l}\text { Partial ITS } \mathrm{X} \\
\text { Sequence }(218 \mathrm{bp})\end{array}$ & $\begin{array}{l}\text { Metschnikowia } \\
\text { bicuspidata }\end{array}$ & U51436 & $\begin{array}{l}3.00 \mathrm{E} \\
-113\end{array}$ & 99 & Metschnikowia bicuspidata & Metschnikowia sp. \\
\hline $\begin{array}{l}\text { Q5S-(1)- } \\
\text { PDA }\end{array}$ & $\begin{array}{l}\text { Partial ITS } \\
\text { Sequence }(245 \mathrm{bp})\end{array}$ & $\begin{array}{l}\text { Metschnikowia } \\
\text { zobellii }\end{array}$ & U51433 & $\begin{array}{l}1.00 \mathrm{E} \\
-91\end{array}$ & 89 & $\begin{array}{l}\text { Omitted from Phylogenetic } \\
\text { Tree due to Poor Quality }\end{array}$ & Metschnikowia sp. \\
\hline R1M-PDA & $\begin{array}{l}\text { Partial ITS } \mathrm{X} \\
\text { Sequence }(218 \mathrm{bp})\end{array}$ & $\begin{array}{l}\text { Metschnikowia } \\
\text { bicuspidata }\end{array}$ & EF643581 & $\begin{array}{l}2.00 \mathrm{E} \\
-88\end{array}$ & 100 & Metschnikowia bicuspidata & Metschnikowia sp. \\
\hline R2M-PDA & $\begin{array}{l}\text { Partial ITS }{ }^{X} \\
\text { Sequence }(218 \mathrm{bp})\end{array}$ & $\begin{array}{l}\text { Metschnikowia } \\
\text { bicuspidata }\end{array}$ & U51436 & $\begin{array}{l}7.00 \mathrm{E} \\
-114 \\
\end{array}$ & 99 & Metschnikowia bicuspidata & Metschnikowia sp. \\
\hline R2S-PDA & $\begin{array}{l}\text { Partial ITS } \mathrm{X} \\
\text { Sequence }(218 \mathrm{bp})\end{array}$ & $\begin{array}{l}\text { Metschnikowia } \\
\text { bicuspidata }\end{array}$ & U51436 & $\begin{array}{l}4.00 \mathrm{E} \\
-106\end{array}$ & 99 & Metschnikowia bicuspidata & Metschnikowia sp. \\
\hline R4M-STD & $\begin{array}{l}\text { Partial ITS } \\
\text { Sequence (247 bp) }\end{array}$ & $\begin{array}{l}\text { Metschnikowia } \\
\text { zobellii }\end{array}$ & U51433 & $\begin{array}{l}3.00 \mathrm{E} \\
-118 \\
\end{array}$ & 97 & $\begin{array}{l}\text { Omitted from Phylogenetic } \\
\text { Tree due to Poor Quality }\end{array}$ & Metschnikowia sp. \\
\hline
\end{tabular}




\begin{tabular}{|c|c|c|c|c|c|c|c|}
\hline R5M-PDA & $\begin{array}{l}\text { Partial ITS }{ }^{X} \\
\text { Sequence }(218 \mathrm{bp})\end{array}$ & $\begin{array}{l}\text { Metschnikowia } \\
\text { bicuspidata }\end{array}$ & U51436 & $\begin{array}{l}9.00 \mathrm{E} \\
-113\end{array}$ & 99 & Metschnikowia bicuspidata & Metschnikowia sp. \\
\hline S1M-PDA & $\begin{array}{l}\text { Partial ITS } \mathrm{X} \\
\text { Sequence }(218 \mathrm{bp})\end{array}$ & $\begin{array}{l}\text { Metschnikowia } \\
\text { bicuspidata }\end{array}$ & U51436 & $\begin{array}{l}4.00 \mathrm{E} \\
-111\end{array}$ & 99 & Metschnikowia bicuspidata & Metschnikowia sp. \\
\hline S1M-STD & $\begin{array}{l}\text { Partial ITS } X \\
\text { Sequence }(218 \mathrm{bp})\end{array}$ & $\begin{array}{l}\text { Metschnikowia } \\
\text { bicuspidata }\end{array}$ & U51436 & $\begin{array}{l}5.00 \mathrm{E} \\
-105\end{array}$ & 100 & Metschnikowia bicuspidata & Metschnikowia sp. \\
\hline S2S-PDA & Contig (383 bp) & $\begin{array}{l}\text { Metschnikowia } \\
\text { zobellii }\end{array}$ & U51433 & $\begin{array}{l}9.00 \mathrm{E} \\
-170 \\
\end{array}$ & 98 & Metschnikowia zobellii & $\begin{array}{l}\text { Metschnikowia } \\
\text { zobellii }\end{array}$ \\
\hline S3M-PDA & $\begin{array}{l}\text { Partial ITS } X \\
\text { Sequence }(218 \mathrm{bp})\end{array}$ & $\begin{array}{l}\text { Metschnikowia } \\
\text { bicuspidata }\end{array}$ & U51436 & $\begin{array}{l}1.00 \mathrm{E} \\
-101 \\
\end{array}$ & 100 & Metschnikowia bicuspidata & Metschnikowia sp. \\
\hline S5S-PDA & $\begin{array}{l}\text { Partial ITS } \\
\text { Sequence (223 bp) }\end{array}$ & $\begin{array}{l}\text { Metschnikowia } \\
\text { bicuspidata }\end{array}$ & U51436 & $\begin{array}{l}3.00 \mathrm{E} \\
-108\end{array}$ & 98 & $\begin{array}{l}\text { Omitted from Phylogenetic } \\
\text { Tree due to Poor Quality }\end{array}$ & Metschnikowia sp. \\
\hline $\begin{array}{l}\text { U3S-1- } \\
\text { STD }\end{array}$ & $\begin{array}{l}\text { Partial ITS } \\
\text { Sequence (203 bp) }\end{array}$ & $\begin{array}{l}\text { Metschnikowia } \\
\text { bicuspidata }\end{array}$ & U51436 & $\begin{array}{l}8.00 \mathrm{E} \\
-98 \\
\end{array}$ & 99 & $\begin{array}{l}\text { Metschnikowia bicuspidata } \\
\text { (Possibly var. bicuspidata or } \\
\text { var. chathamia) }\end{array}$ & Metschnikowia sp. \\
\hline U5S-PDA & $\begin{array}{l}\text { Partial ITS } \\
\text { Sequence }(225 \mathrm{bp})\end{array}$ & $\begin{array}{l}\text { Metschnikowia } \\
\text { bicuspidata }\end{array}$ & U51436 & $\begin{array}{l}6.00 \mathrm{E} \\
-110\end{array}$ & 99 & $\begin{array}{l}\text { Metschnikowia bicuspidata } \\
\text { (Possibly var. bicuspidata or } \\
\text { var. chathamia) }\end{array}$ & Metschnikowia sp. \\
\hline X21S-STD & $\begin{array}{l}\text { Partial ITS } \\
\text { Sequence (227 bp) }\end{array}$ & $\begin{array}{l}\text { Metschnikowia } \\
\text { zobellii }\end{array}$ & U51433 & $\begin{array}{l}3.00 \mathrm{E} \\
-98\end{array}$ & 93 & $\begin{array}{l}\text { Omitted from Phylogenetic } \\
\text { Tree due to Poor Quality }\end{array}$ & Metschnikowia sp. \\
\hline Y2S-STD & $\begin{array}{l}\text { Partial ITS } \\
\text { Sequence (237 bp) }\end{array}$ & $\begin{array}{l}\text { Metschnikowia } \\
\text { zobellii }\end{array}$ & U51433 & $\begin{array}{l}8.00 \mathrm{E} \\
-104\end{array}$ & 94 & $\begin{array}{l}\text { Omitted from Phylogenetic } \\
\text { Tree due to Poor Quality }\end{array}$ & Metschnikowia sp. \\
\hline \multicolumn{8}{|c|}{ Agaricomycotina } \\
\hline V5M-PDA & Contig (507 bp) & Bullera unica & AF444441 & 0 & 100 & Bullera unica & Bullera unica \\
\hline Z2S-STD & Contig (473 bp) & $\begin{array}{l}\text { Cryptococcus } \\
\text { sp. }\end{array}$ & HM849618 & 0 & 100 & $\begin{array}{l}\text { Uncultured Fungus } \\
\text { AM901777 (Second Closest } \\
\text { Organism was Cryptococcus } \\
\text { carnescens) }\end{array}$ & $\begin{array}{l}\text { Cryptococcus } \\
\text { carnescens }\end{array}$ \\
\hline U3S-PDA & Contig (603 bp) & $\begin{array}{l}\text { Cryptococcus } \\
\text { chernovii }\end{array}$ & AF444354 & 0 & 99 & $\begin{array}{l}\text { Uncultured Fungus AJ875389 } \\
\text { (Second Closest Organism } \\
\text { was Cryptococcus chernovii) }\end{array}$ & $\begin{array}{l}\text { Cryptococcus } \\
\text { chernovii }\end{array}$ \\
\hline
\end{tabular}




\begin{tabular}{|c|c|c|c|c|c|c|c|}
\hline Y5S-PDA & Contig (460 bp) & $\begin{array}{l}\text { Uncultured } \\
\text { Basidiomycete }\end{array}$ & AM901805 & 0 & 100 & $\begin{array}{l}\text { Uncultured Fungus } \\
\text { AM901805 } \\
\text { (Second Closest Organism } \\
\text { was Cryptococcus dimennae) }\end{array}$ & $\begin{array}{l}\text { Cryptococcus } \\
\text { dimennae }\end{array}$ \\
\hline $\begin{array}{l}\text { T1S-3- } \\
\text { PDA }\end{array}$ & Contig (421 bp) & $\begin{array}{l}\text { Uncultured } \\
\text { Basidiomycete }\end{array}$ & AM901805 & 0 & 100 & $\begin{array}{l}\text { Uncultured Fungus } \\
\text { AM901805 } \\
\text { (Second Closest Organism } \\
\text { was Cryptococcus dimennae) }\end{array}$ & $\begin{array}{l}\text { Cryptococcus } \\
\text { dimennae }\end{array}$ \\
\hline $\begin{array}{l}\text { Z4S-1- } \\
\text { STD } \\
\end{array}$ & Contig (455 bp) & $\begin{array}{l}\text { Uncultured } \\
\text { Basidiomycete }\end{array}$ & AM901805 & 0 & 99 & $\begin{array}{l}\text { Uncultured Fungus } \\
\text { AM901805 } \\
\text { (Second Closest Organism } \\
\text { was Cryptococcus dimennae) }\end{array}$ & $\begin{array}{l}\text { Cryptococcus } \\
\text { dimennae }\end{array}$ \\
\hline P4M-STD & Contig (582 bp) & $\begin{array}{l}\text { Uncultured } \\
\text { Fungus }\end{array}$ & AB520402 & 0 & 100 & Cryptococcus macerans & $\begin{array}{l}\text { Cryptococcus } \\
\text { macerans }\end{array}$ \\
\hline $\begin{array}{l}\text { T1S-1- } \\
\text { PDA }\end{array}$ & Contig (582 bp) & $\begin{array}{l}\text { Cystofilobasidium } \\
\text { macerans } \\
\text { (A Teleomorph of } \\
\text { Cryptococcus } \\
\text { macerans) }\end{array}$ & AF444317 & 0 & 100 & Cryptococcus macerans & $\begin{array}{l}\text { Cryptococcus } \\
\text { macerans }\end{array}$ \\
\hline S4S-PDA & Contig (481 bp) & $\begin{array}{l}\text { Cryptococcus } \\
\text { victoriae }\end{array}$ & AY040654 & 0 & 100 & Cryptococcus victoriae & $\begin{array}{l}\text { Cryptococcus } \\
\text { victoriae }\end{array}$ \\
\hline $\begin{array}{l}\text { W5S-4- } \\
\text { STD }\end{array}$ & Contig (480 bp) & $\begin{array}{l}\text { Cryptococcus } \\
\text { sp. }\end{array}$ & HM589279 & 0 & 100 & $\begin{array}{l}\text { Cryptococcus sp. } \\
\text { (Second Closest Organism } \\
\text { was Cryptococcus victoriae) }\end{array}$ & $\begin{array}{l}\text { Cryptococcus } \\
\text { victoriae }\end{array}$ \\
\hline $\begin{array}{l}\text { L2S-1- } \\
\text { PDA }\end{array}$ & Contig (481 bp) & $\begin{array}{l}\text { Cryptococcus } \\
\text { sp. }\end{array}$ & HM589279 & 0 & 100 & $\begin{array}{l}\text { Cryptococcus sp. } \\
\text { (Second Closest Organism } \\
\text { was Cryptococcus victoriae) }\end{array}$ & $\begin{array}{l}\text { Cryptococcus } \\
\text { victoriae }\end{array}$ \\
\hline Y2M-PDA & Contig (460 bp) & $\begin{array}{l}\text { Uncultured } \\
\text { Basidiomycete }\end{array}$ & AM901805 & 0 & 100 & $\begin{array}{l}\text { Unresolved via Phylogenetic } \\
\text { tree }\end{array}$ & $\begin{array}{l}\text { Cryptococcus } \\
\text { sp. }\end{array}$ \\
\hline \multicolumn{8}{|c|}{ Pucciniomycotina } \\
\hline H10S-PDA & Contig (599 bp) & Kondoa aeria & AF444595 & 0 & 99 & Kondoa aeria & Kondoa aeria \\
\hline B4M-STD & Contig (542 bp) & $\begin{array}{l}\text { Rhodotorula } \\
\text { minuta }\end{array}$ & AB078501 & 0 & 100 & Rhodotorula minuta & $\begin{array}{l}\text { Rhodotorula } \\
\text { minuta }\end{array}$ \\
\hline
\end{tabular}




\begin{tabular}{|l|l|l|l|l|l|l|l|}
\hline E3M-STD & Contig (576 bp) & $\begin{array}{l}\text { Rhodotorula } \\
\text { mucilaginosa }\end{array}$ & HQ702343 & 0 & 100 & Rhodotorula mucilaginosa & $\begin{array}{l}\text { Rhodotorula } \\
\text { mucilaginosa }\end{array}$ \\
\hline E5S-PDA & Contig (571 bp) & $\begin{array}{l}\text { Rhodotorula } \\
\text { mucilaginosa }\end{array}$ & EU871492 & 0 & 99 & Rhodotorula mucilaginosa \\
\hline $\begin{array}{l}\text { Q5S-2- } \\
\text { PDA }\end{array}$ & Contig (572 bp) & $\begin{array}{l}\text { Rhodotorula } \\
\text { mucilaginosa }\end{array}$ & HQ702343 & 0 & 100 & Rhodotorula \\
I1-STD & Contig (543 bp) & $\begin{array}{l}\text { Rhodotorula } \\
\text { slooffiae }\end{array}$ & AB025994 & 0 & 99 & Rhodotorula slooffiae & $\begin{array}{l}\text { Rhodotorula } \\
\text { mucilaginosa }\end{array}$ \\
\hline J3S-STD & Contig (543 bp) & $\begin{array}{l}\text { Rhodotorula } \\
\text { slooffiae }\end{array}$ & AF444589 & 0 & 100 & Rhodotorula slooffiae & $\begin{array}{l}\text { Rhodotorula } \\
\text { slooffiae }\end{array}$ \\
\hline P2S-PDA & Contig (539 bp) & Rhodotorula sp. & FJ238090 & 0 & 99 & Rhodotorula sp. & Rhodotorula sp. \\
\hline $\begin{array}{l}\text { Z4S-1- } \\
\text { PDA }\end{array}$ & Contig (564 bp) & $\begin{array}{l}\text { Sakaguchia } \\
\text { dacryoidea }\end{array}$ & AF444597 & 0 & 98 & Sakaguchia dacryoidea & $\begin{array}{l}\text { Sakaguchia } \\
\text { dacryoidea }\end{array}$ \\
\hline
\end{tabular}

$\mathrm{X}$ denotes 25 individual and identical partial ITS sequences which had Metschnikowia bicuspidata (possible varieties include

Metschnikowia bicuspidata var. bicuspidata and Metschnikowia bicuspidata var. chathamia) as their closest phylogenetic neighbour. 


\subsection{Ascomycota}

\subsubsection{Isolates Identified}

A total of 53 isolates were identified from the Ascomycota. Three isolates were identified as Aureobasidium pullulans (De Bary) G. Arnaud ex Cif., Ribaldi \& Corte in the subphylum Pezizomycotina. Fifty isolates were identified from the genus Metschnikowia in the subphylum Saccharomycotina.

\subsubsection{Pezizomycotina}

\subsubsection{Identification via Molecular Techniques}

Three isolates were identified as the yeast-like fungus Aureobasidium pullulans in the subphylum Pezizomycotina (Table 2). ITS sequences obtained from Pezizomycotina isolates were of good quality and formed contigs ranging from 526 to 541 bp in length. The ITS contig from each isolate was queried against GenBank and all three gave excellent sequence identity (100\%) to an Uncultured Dothioraceae (FM178237), Aureobasidium pullulans (HM992505, HQ115720 and HM849619) and an Uncultured Fungus (GQ851743). A Geneious alignment also confirmed that all three isolates shared a pairwise identity of $100 \%$ with the above ITS sequences, so 
construction of a phylogenetic tree was unnecessary (Appendix 5.3). Results from GenBank searches and the Geneious alignment were in agreement, allowing confident identification of all three isolates as Aureobasidium pullulans.

\subsubsection{Morphological Observations}

All three isolates from the Pezizomycotina had circular, peach coloured colonies with a slightly raised shiny surface and a filamentous margin (Fig. 3 and Table 3). Microscopically, the cells of all three isolates were cylindrical and two isolates displayed clustering or formation of chains of cells (as indicated in Fig. 3). Isolates from the Pezizomycotina had the largest cells in this investigation, ranging from 9.8 to $11.8 \mu \mathrm{m}$, with an average of $10.7 \mu \mathrm{m}$ (Table 3). Descriptions of Aureobasidium pullulans from the literature were generally in agreement with observations made during this investigation, thus, data from culture studies support the identification of these isolates (CBS-KNAW 2012; MycoBank 2012). Although not technically a yeast, the yeast-like appearance of Aureobasidium pullulans (Zalar et al. 2008) may be responsible for its isolation during this investigation. 
Table 3. Morphology of Pezizomycotina isolates.

\begin{tabular}{|l|l|l|l|l|l|l|l|l|}
\hline \multicolumn{1}{|c|}{$\begin{array}{c}\text { Assigned } \\
\text { Organism }\end{array}$} & \multicolumn{1}{|c|}{$\begin{array}{c}\text { Isolate } \\
\text { Code }\end{array}$} & $\begin{array}{c}\text { Alga of } \\
\text { Origin }\end{array}$ & $\begin{array}{l}\text { Colony } \\
\text { Colour }\end{array}$ & $\begin{array}{l}\text { Colony } \\
\text { Surface }\end{array}$ & $\begin{array}{c}\text { Additional Notes: } \\
\text { Colony Appearance }\end{array}$ & $\begin{array}{c}\text { Average } \\
\text { Cell Size } \\
(\mu \mathrm{m})\end{array}$ & $\begin{array}{c}\text { Cell Shape } \\
\text { Additional Notes: } \\
\text { Cellular } \\
\text { Appearance }\end{array}$ \\
\hline $\begin{array}{l}\text { Aureobasidium } \\
\text { pullulans }\end{array}$ & L10S-PDA & $\begin{array}{l}\text { Cystophora } \\
\text { scalaris }\end{array}$ & Peach & Shiny & $\begin{array}{l}\text { Slightly Raised with } \\
\text { Filamentous Margin }\end{array}$ & 11.8 & Cylindrical & Cluster Formation \\
\hline $\begin{array}{l}\text { Aureobasidium } \\
\text { pullulans }\end{array}$ & N2M-PDA & $\begin{array}{l}\text { Caulerpa } \\
\text { brownii }\end{array}$ & Peach & Shiny & $\begin{array}{l}\text { Slightly Raised with } \\
\text { Filamentous Margin }\end{array}$ & 10.6 & Cylindrical \\
\hline $\begin{array}{l}\text { Aureobasidium } \\
\text { pullulans }\end{array}$ & P5S-PDA & $\begin{array}{l}\text { Macrocystis } \\
\text { pyrifera }\end{array}$ & Peach & Shiny & $\begin{array}{l}\text { Slightly Raised with } \\
\text { Filamentous Margin }\end{array}$ & 9.8 & Cylindrical & Cluster Formation \\
\hline
\end{tabular}




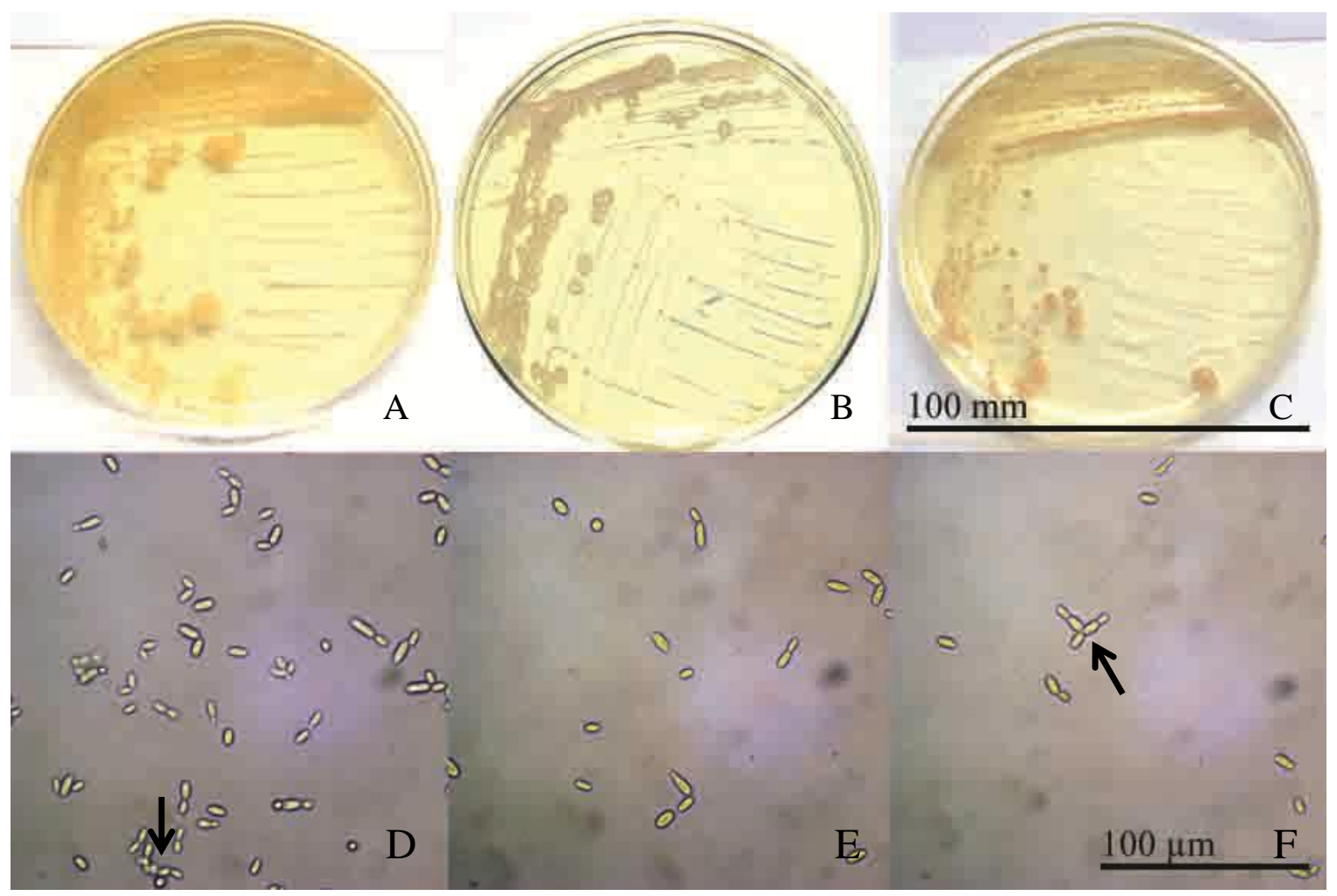

Figure 3. A, B, C: Photographs of colonies formed by Pezizomycotina isolates on agar plates. $A=$ L10S-PDA, B=N2M-PDA, C=P5S-PDA. D, E, F: Corresponding microscopic images of the same Pezizomycotina isolates. D=L10S-PDA, E=N2M-PDA, F=P5S-PDA. All isolates were identified as Aureobasidium pullulans. 


\subsubsection{Saccharomycotina}

\subsubsection{Identification via Molecular Techniques}

Fifty isolates were identified from the genus Metschnikowia in the subphylum Saccharomycotina (Table 2). A contig (383 bp) was formed by ITS sequences from only one Saccharomycotina isolate. For the remaining 49 isolates, the ITS4 primer side yielded some partial sequences (159 to $247 \mathrm{bp}$ ) which were sufficient for use in molecular identification procedures. The partial ITS sequence or contig from each isolate was queried against GenBank and gave good sequence identity (89-100\%) to Metschnikowia bicuspidata, Metschnikowia zobellii or Metschnikowia reukaufii (Pitt \& M.W. Mill.).

A phylogenetic tree was constructed with ITS sequences from Saccharomycotina isolates plus selected sequences from GenBank and the literature (Fig. 4). Overall, the Saccharomycotina phylogenetic tree did not demonstrate good separation of species. One isolate (I2-STD) was unresolved and identified as Metschnikowia sp. An unsupported group contained sequences from isolates obtained during this investigation plus sequences from Metschnikowia bicuspidata and its varieties: Metschnikowia bicuspidata var. bicuspidata and Metschnikowia bicuspidata var. chathamia. Isolates situated in this unsupported group were $\mathrm{X}$ (which represents 25 individual isolates with identical partial ITS sequences), KK5S-STD, U5S-PDA and U3S-1-STD. All isolates in this unsupported group were suspected to be from the species Metschnikowia bicuspidata (or possibly one of its varieties: Metschnikowia bicuspidata var. bicuspidata or 
Metschnikowia bicuspidata var. chathamia), but were identified as Metschnikowia sp. due to the lack of support. Sequences from U5S-PDA and U3S-1-STD formed a supported group by themselves (bootstrap support $88 \%$ ). Only two isolates in the Saccharomycotina phylogenetic tree were resolved to a species level: S2S-PDA and N5M-STD formed a well-supported group (bootstrap support $98 \%$ ) with two Metschnikowia zobellii sequences (U51433 and CBS4821). N5M-STD had a partial ITS sequence of $245 \mathrm{bp}$ and S2S-PDA had an ITS contig of $383 \mathrm{bp}$. The poor resolution of species in the Saccharomycotina phylogenetic tree did not contradict or support results obtained from GenBank searches.

\subsubsection{Morphological Observations}

All isolates from the Saccharomycotina had circular, cream coloured colonies with an entire margin (Fig. 5 and Table 4). Colony surface was glistening, shiny or heterogeneous (both shiny and matt colony textures were apparent). The majority of colonies appeared to have a slightly raised or convex surface, although some were flat and spread out. The cell morphology of Saccharomycotina isolates was variable; most isolates had an ellipsoidal cell shape, some were elongated ellipsoids (Fig. 6), and many formed clusters or chains of cells (some of which are indicated in Fig. 6). Cell size ranged from 3.8 to $6.0 \mu \mathrm{m}$, with an average of $4.8 \mu \mathrm{m}$ (Table 4). Literary descriptions of yeasts from the Metschnikowia were generally in agreement with observations made during this investigation, supporting the identification of these isolates as Metschnikowia sp. or Metschnikowia zobellii (CBS-KNAW 2012; MycoBank 2012). 


\subsubsection{Isolate Identification Summary}

ITS sequence data and supporting morphological observations enabled confident species identification of just two isolates from the Saccharomycotina: both isolates were identified as Metschnikowia zobellii (Table 2). The remaining 48 isolates were identified as Metschnikowia sp. and included 28 isolates that formed an unsupported phylogenetic group with Metschnikowia bicuspidata (all 28 isolates had partial ITS sequences, 25 of which were identical) plus 19 isolates omitted from phylogenetic tree construction due to poor quality partial ITS sequences. 


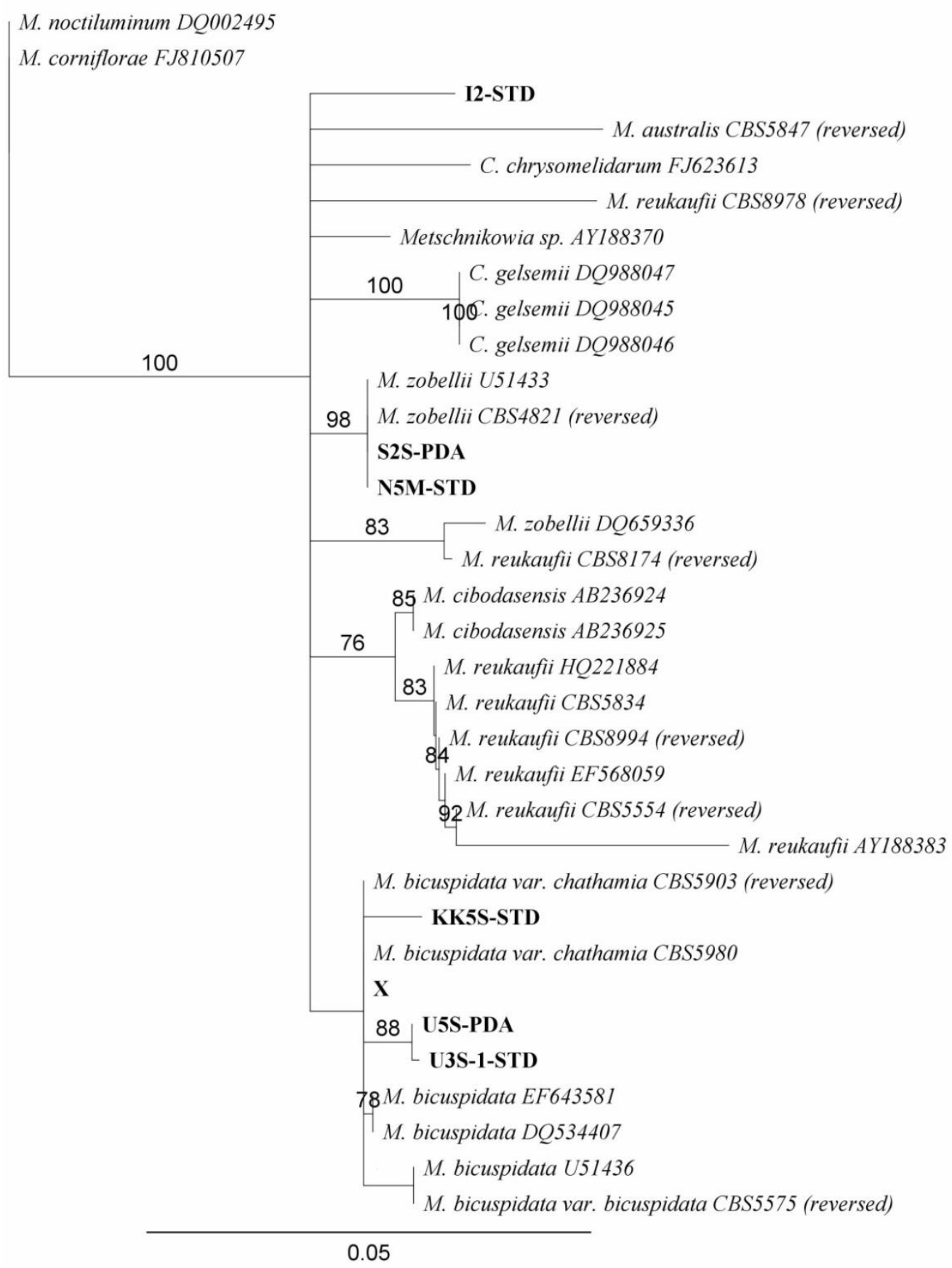

Figure 4. Neighbour-joining phylogenetic tree based on ITS sequences from Saccharomycotina isolates (shown in bold), using the Tamura-Nei model. Bootstrap values are shown for nodes supported by $>70 \%$ of 100 replicates. Scale bar represents 0.05 substitutions per site. For simplicity, $\mathrm{X}$ represents 25 individual isolates with identical partial ITS sequences. 
Table 4. Morphology of Saccharomycotina isolates.

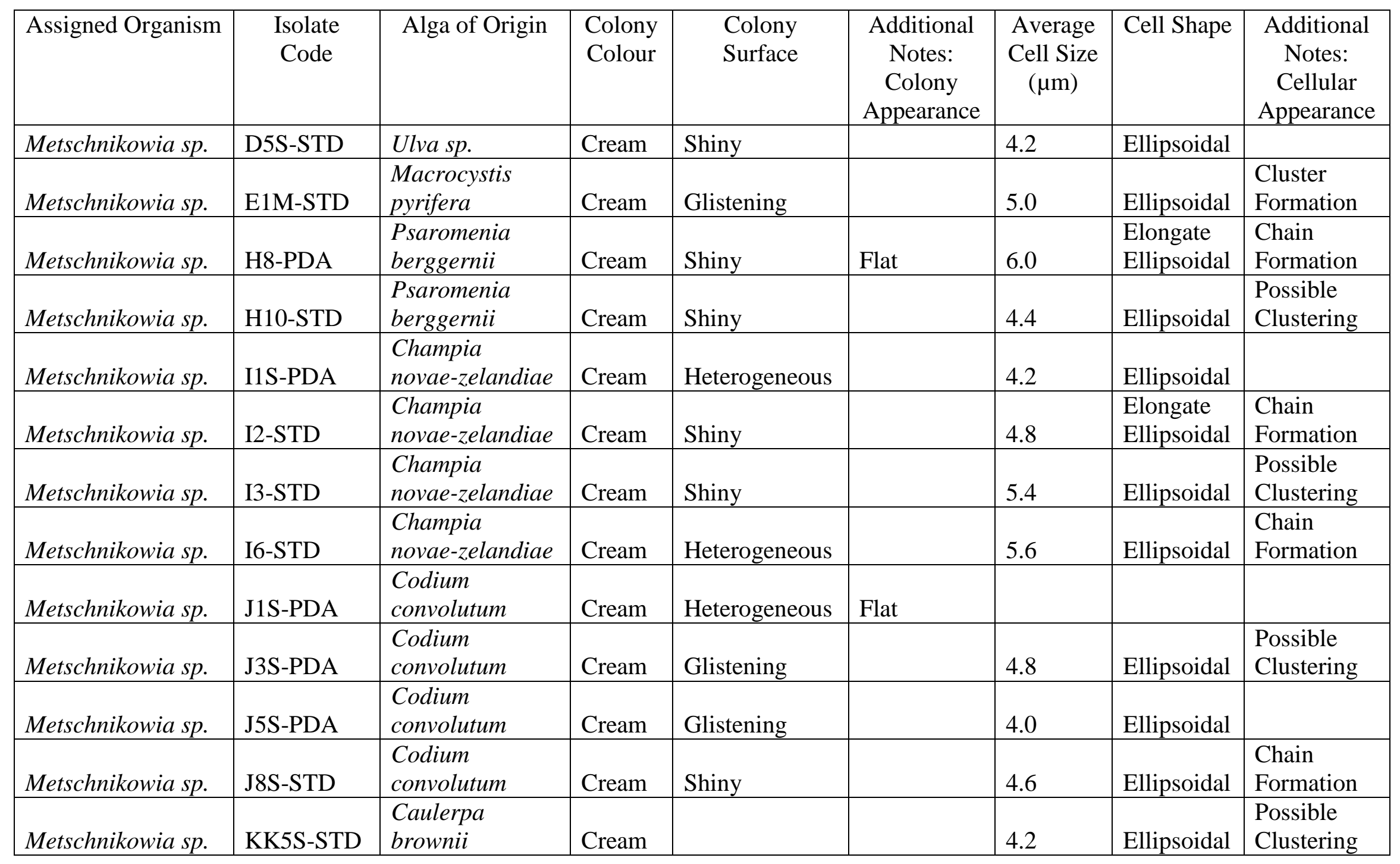




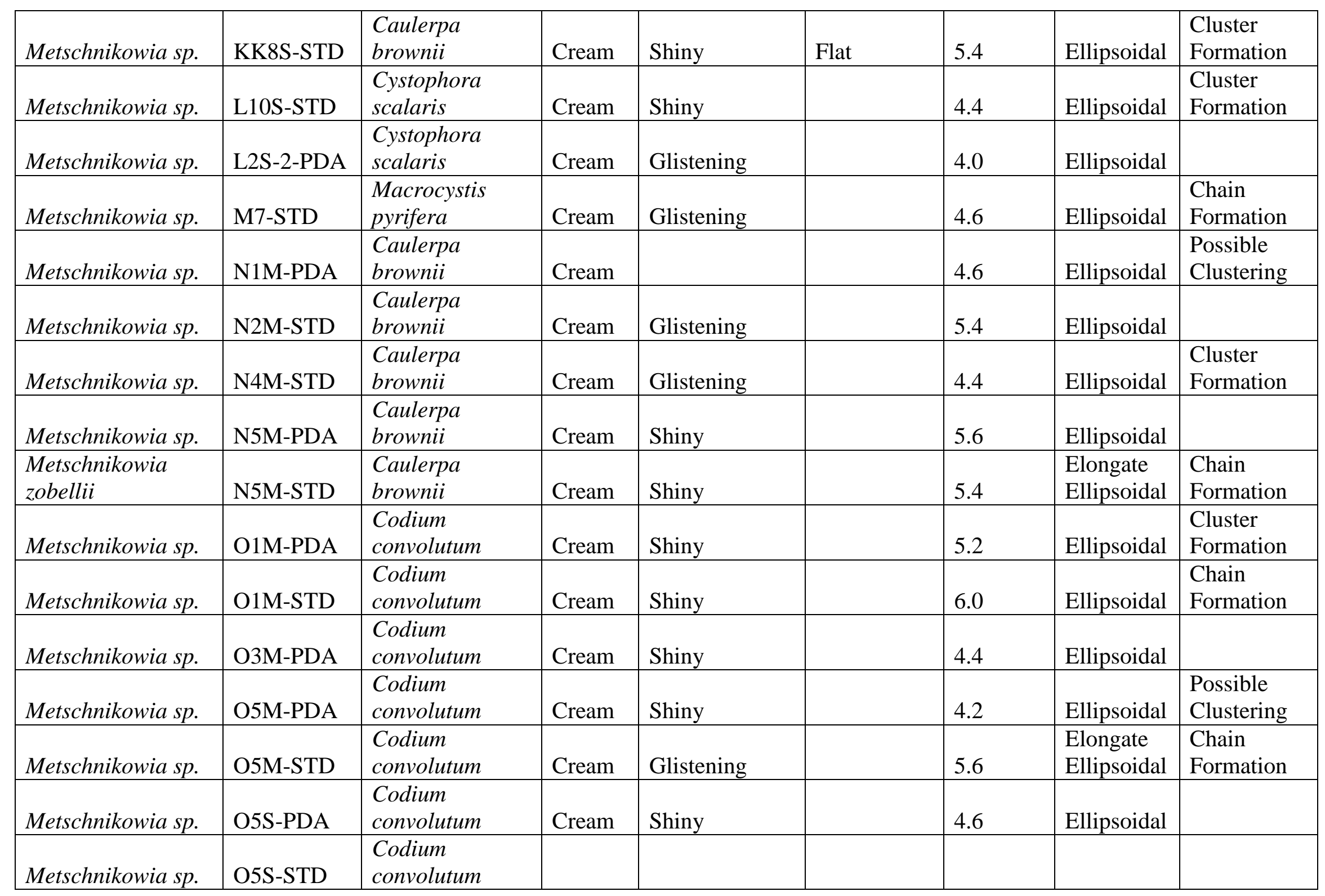




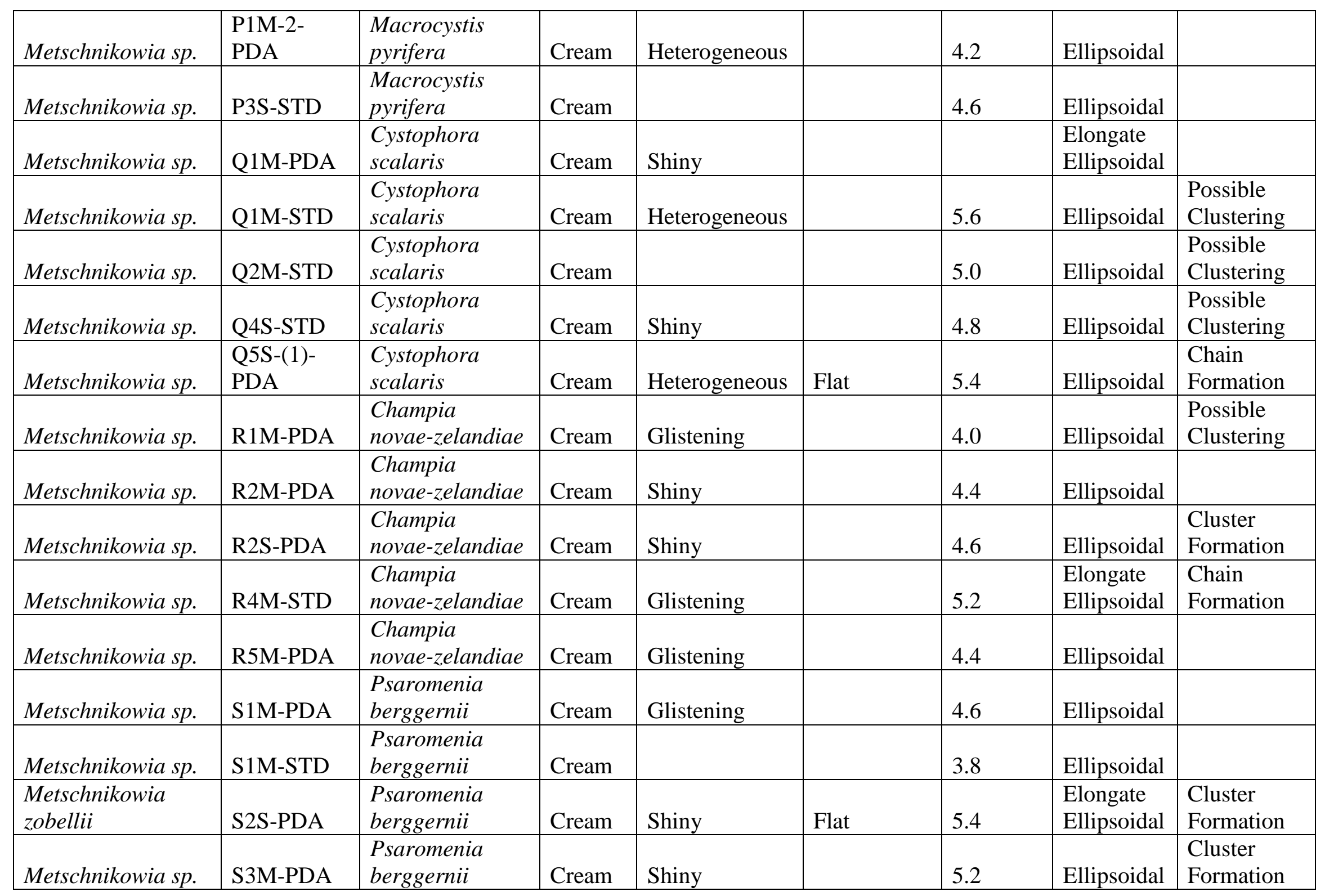




\begin{tabular}{|c|c|c|c|c|c|c|c|}
\hline Metschnikowia sp. & S5S-PDA & $\begin{array}{l}\text { Psaromenia } \\
\text { berggernii }\end{array}$ & Cream & Shiny & 4.4 & Ellipsoidal & \\
\hline Metschnikowia sp. & U3S-1-STD & $\begin{array}{l}\text { Cystophora } \\
\text { scalaris }\end{array}$ & Cream & Glistening & 4.4 & Ellipsoidal & \\
\hline Metschnikowia sp. & U5S-PDA & $\begin{array}{l}\text { Cystophora } \\
\text { scalaris }\end{array}$ & Cream & Glistening & 4.0 & Ellipsoidal & $\begin{array}{l}\text { Possible } \\
\text { Clustering }\end{array}$ \\
\hline Metschnikowia sp. & X21S-STD & $\begin{array}{l}\text { Psaromenia } \\
\text { berggernii }\end{array}$ & Cream & Glistening & 5.0 & $\begin{array}{l}\text { Elongate } \\
\text { Ellipsoidal }\end{array}$ & $\begin{array}{l}\text { Chain } \\
\text { Formation }\end{array}$ \\
\hline Metschnikowia sp. & Y2S-STD & $\begin{array}{l}\text { Codium } \\
\text { convolutum }\end{array}$ & Cream & Heterogeneous & 5.0 & $\begin{array}{l}\text { Elongate } \\
\text { Ellipsoidal }\end{array}$ & $\begin{array}{l}\text { Chain } \\
\text { Formation }\end{array}$ \\
\hline
\end{tabular}




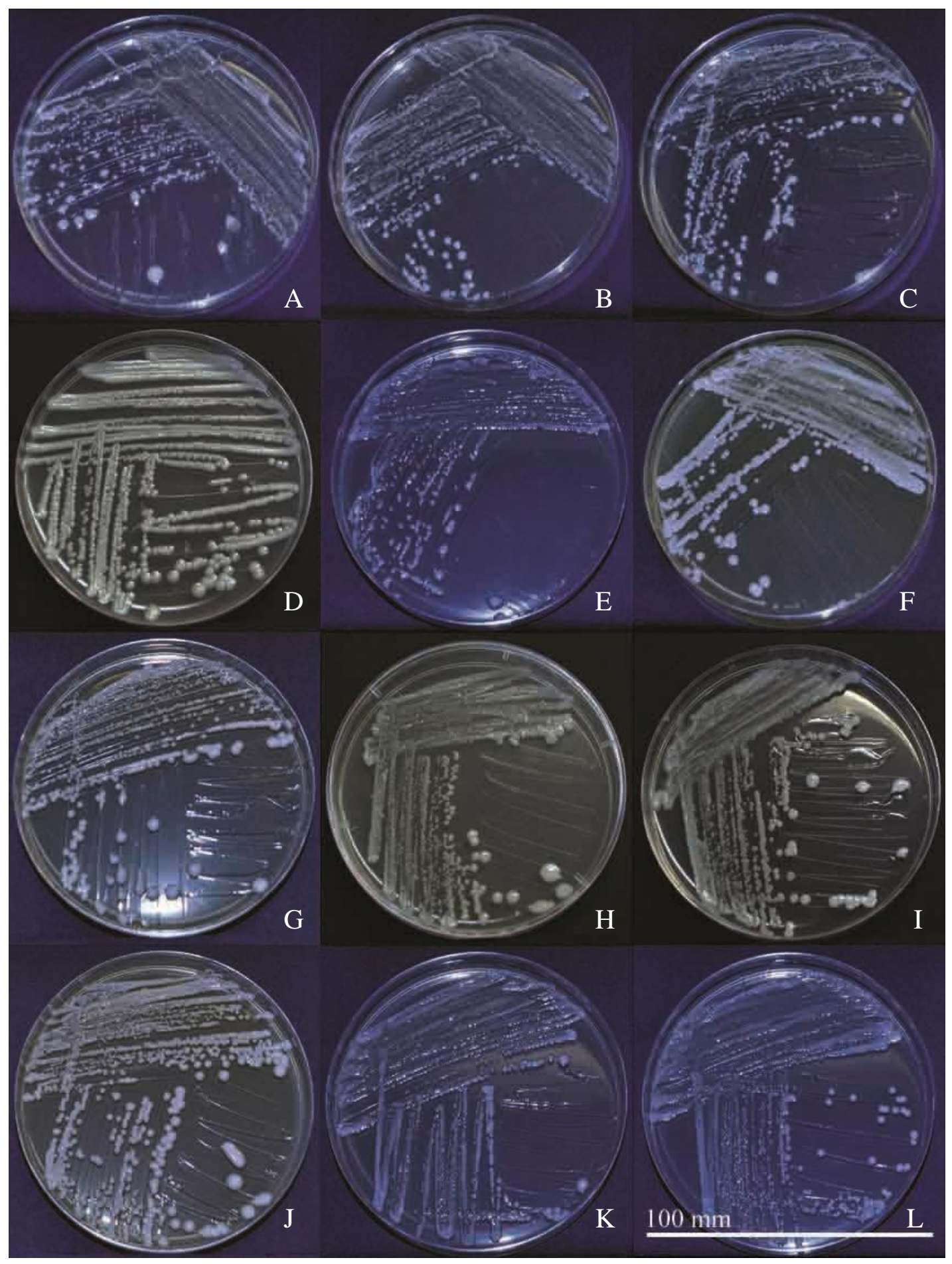

Figure 5. Photographs of colonies formed by Saccharomycotina isolates on agar plates. A=J3SPDA, B=J5S-PDA, C= L2S-2-PDA, D=O3M-PDA, E=R5M-PDA, F=S2S-PDA, G=D5S-STD, $\mathrm{H}=\mathrm{I} 3-\mathrm{STD}, \mathrm{I}=\mathrm{I} 6-\mathrm{STD}, \mathrm{J}=\mathrm{L} 10 \mathrm{~S}-\mathrm{STD}, \mathrm{K}=\mathrm{N} 2 \mathrm{M}-\mathrm{STD}, \mathrm{L}=\mathrm{N} 4 \mathrm{M}-\mathrm{STD}$. All isolates were identified as Metschnikowia sp., except for S2S-PDA which was identified as Metschnikowia zobellii. 


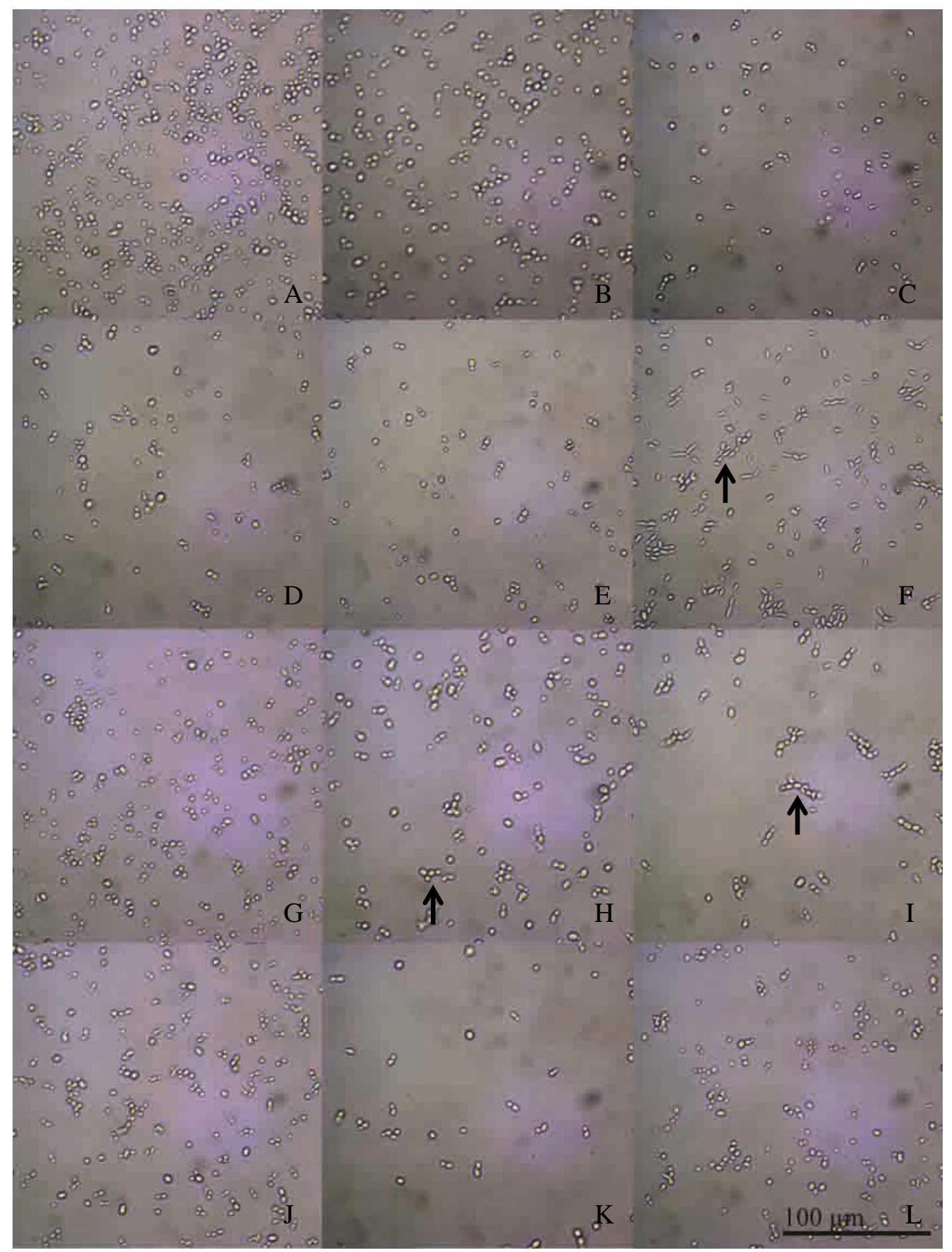

Figure 6. Microscopic images of Saccharomycotina isolates. A=J3S-PDA, B=J5S-PDA, C=L2S-

2-PDA， D=O3M-PDA， E=R5M-PDA， F=S2S-PDA， G=D5S-STD， H=I3-STD， I=I6-STD, $\mathrm{J}=\mathrm{L} 10 \mathrm{~S}-\mathrm{STD}, \mathrm{K}=\mathrm{N} 2 \mathrm{M}-\mathrm{STD}, \mathrm{L}=\mathrm{N} 4 \mathrm{M}-\mathrm{STD}$. All isolates were identified as Metschnikowia sp., except for S2S-PDA which was identified as Metschnikowia zobellii. 


\subsection{Basidiomycota}

\subsubsection{Isolates Identified}

A total of 21 isolates were identified from the Basidiomycota. Twelve isolates were identified from a range of species in the subphylum Agaricomycotina, and nine isolates were identified from the subphylum Pucciniomycotina.

\subsubsection{Agaricomycotina}

\subsubsection{Identification via Molecular Techniques}

Twelve isolates were identified from the subphylum Agaricomycotina (Table 2). These were comprised of 11 isolates from the genus Cryptococcus and a single isolate of Bullera unica (Hamam. \& Nakase). ITS sequences obtained from Agaricomycotina isolates were of good quality and formed contigs ranging from 421 to 603 bp in length. The ITS contig from each isolate was queried against GenBank and gave excellent sequence identity $(99-100 \%)$ to a named Cryptococcus species, Cryptococcus sp., Bullera unica or an uncultured fungus. Contigs without a named species as their primary GenBank result typically demonstrated excellent sequence identity $(99-100 \%)$ to another named Cryptococcus species. 
A phylogenetic tree was constructed with ITS sequences from Agaricomycotina isolates plus selected sequences from GenBank and the literature (Fig. 7). Overall, the Agaricomycotina phylogenetic tree demonstrated good separation of ITS sequences from named species and Agaricomycotina isolates into distinct clades. Sequences from uncultured fungi were also distributed within these clades and are not mentioned further as they did not contribute to isolate identification. One isolate (V5M-PDA) was in a well-supported clade with, and identified as, Bullera unica (bootstrap support $89 \%$ ). Two isolates (P4M-STD and T1S-1-PDA) in a wellsupported clade with Cryptococcus macerans (Frederiksen) Phaff \& Fell and Cystofilobasidium macerans (a teleomorph of Cryptococcus macerans) (bootstrap support $100 \%$ ) were identified as Cryptococcus macerans. Due to their position in a well-supported clade with the species, three isolates (T1S-3-PDA, Y5S-PDA and Z4S-1-STD) were identified as Cryptococcus dimennae Fell \& Phaff (bootstrap support $100 \%$ ). A further three isolates (L2S-1-PDA, W5S-4-STD and S4SPDA) were in a well-supported clade with, and identified as, Cryptococcus victoriae (M.J. Montes et al.) (bootstrap support $99 \%$ ). Although it was in an unsupported clade, one isolate (Z2S-STD) was identified as Cryptococcus carnescens (Verona \& Luchetti) Takash., Sugita, Shinoda \& Nakase. Finally, a well-supported clade (bootstrap support $100 \%$ ) consisted of an unsupported and a moderately supported group of sequences. The unsupported group contained sequences from Cryptococcus magnus (Lodder \& Kreger-van Rij) Baptist \& Kurtzman, Cryptococcus oeirensis Á. Fonseca, Scorzetti \& Fell, and one isolate (Y2M-PDA) which was in an unresolved position and identified as Cryptococcus $s p$. The moderately supported group (bootstrap support $79 \%$ ) was comprised of two well-supported clades (bootstrap support $100 \%$ for both clades): one clade contained sequences from Cryptococcus chernovii Á. Fonseca, 
Scorzetti \& Fell, and the other clade contained sequences from uncultured fungi plus an isolate (U3S-PDA) identified as Cryptococcus chernovii. Overall, the Agaricomycotina phylogenetic tree displayed good resolution of isolates to a species level, with the exception of one isolate (Y2M-PDA) which was identified as Cryptococcus sp. Results from this phylogenetic tree were in agreement with those obtained from GenBank searches, and the combination of these two methods allowed confident identification of isolates from the Agaricomycotina.

\subsubsection{Morphological Observations}

All isolates from the Agaricomycotina had circular colonies with an entire margin (Fig. 8 and Table 5). Colonies were shiny or glistening and displayed a range of colours including cream, peach and bright pink. Colony elevation was slightly raised or convex, except for one isolate identified as Cryptococcus macerans which was umbonate (T1S-1-PDA) (B in Fig. 8). The cell morphology of Agaricomycotina isolates was variable; most isolates had an ellipsoidal cell shape, although a small number had elongated ellipsoidal cells (Fig. 9). A couple of isolates displayed what appeared to be a clustering of cells, but distinct chains of cells were not observed (as indicated in Fig. 9). Cell size ranged from 4.2 to $7.8 \mu \mathrm{m}$, with an average of $5.3 \mu \mathrm{m}$ (Table 5). Literary descriptions of yeasts from the Agaricomycotina were generally in agreement with observations made during this investigation, thus, data from culture studies support the identification of these isolates (CBS-KNAW 2012; MycoBank 2012). 


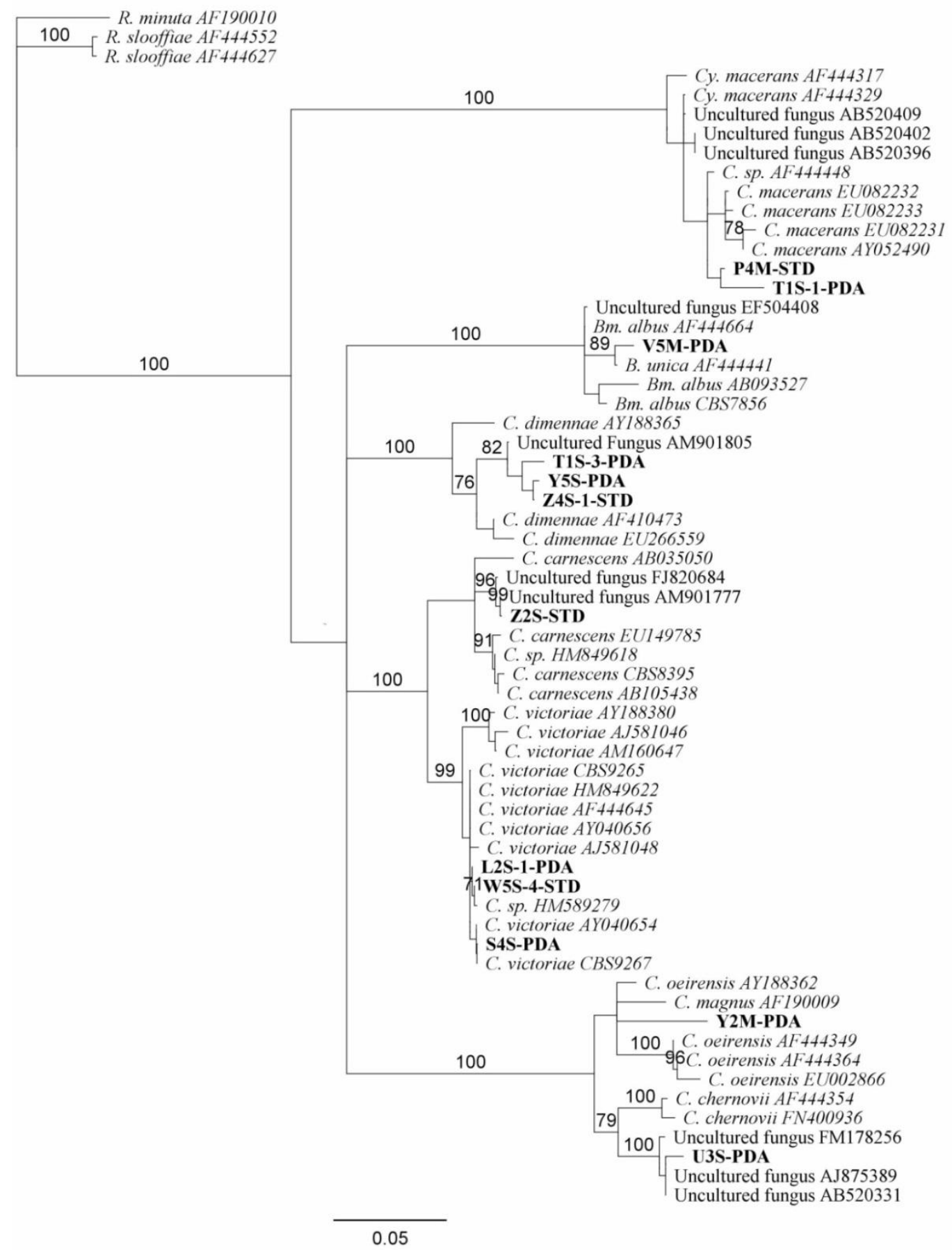

Figure 7. Neighbour-joining phylogenetic tree based on ITS sequences from Agaricomycotina isolates (shown in bold), using the Tamura-Nei model. Bootstrap values are shown for nodes supported by $>70 \%$ of 100 replicates. Scale bar represents 0.05 substitutions per site. The outgroup contains ITS sequences from the genus Rhodotorula. 
Table 5. Morphology of Agaricomycotina isolates.

\begin{tabular}{|c|c|c|c|c|c|c|c|c|}
\hline $\begin{array}{l}\text { Assigned } \\
\text { Organism }\end{array}$ & $\begin{array}{l}\text { Isolate } \\
\text { Code }\end{array}$ & Alga of Origin & $\begin{array}{l}\text { Colony } \\
\text { Colour }\end{array}$ & $\begin{array}{l}\text { Colony } \\
\text { Surface }\end{array}$ & $\begin{array}{l}\text { Additional } \\
\text { Notes: } \\
\text { Colony } \\
\text { Appearance }\end{array}$ & $\begin{array}{c}\text { Average } \\
\text { Cell Size } \\
(\mu \mathrm{m})\end{array}$ & Cell Shape & $\begin{array}{l}\text { Additional } \\
\text { Notes: } \\
\text { Cellular } \\
\text { Appearance }\end{array}$ \\
\hline Bullera unica & V5M-PDA & $\begin{array}{l}\text { Cladhymenia } \\
\text { oblongifolia }\end{array}$ & Peach & Shiny & & 5.0 & Ellipsoidal & \\
\hline $\begin{array}{l}\text { Cryptococcus } \\
\text { carnescens }\end{array}$ & Z2S-STD & Caulerpa brownii & Peach & Glistening & & 4.4 & Ellipsoidal & \\
\hline $\begin{array}{l}\text { Cryptococcus } \\
\text { chernovii }\end{array}$ & U3S-PDA & Cystophora scalaris & Cream & & & 5.2 & Ellipsoidal & \\
\hline $\begin{array}{l}\text { Cryptococcus } \\
\text { dimennae }\end{array}$ & T1S-3-PDA & Macrocystis pyrifera & Peach & Shiny & & 4.2 & Ellipsoidal & \\
\hline $\begin{array}{l}\text { Cryptococcus } \\
\text { dimennae }\end{array}$ & Y5S-PDA & Codium convolutum & Peach & Shiny & & 4.4 & Ellipsoidal & \\
\hline $\begin{array}{l}\text { Cryptococcus } \\
\text { dimennae }\end{array}$ & Z4S-1-STD & Caulerpa brownii & Peach & Glistening & & 5.0 & Ellipsoidal & $\begin{array}{l}\text { Possible } \\
\text { Clustering }\end{array}$ \\
\hline $\begin{array}{l}\text { Cryptococcus } \\
\text { macerans }\end{array}$ & P4M-STD & Macrocystis pyrifera & $\begin{array}{l}\text { Bright } \\
\text { Pink }\end{array}$ & & & 7.8 & $\begin{array}{l}\text { Elongate } \\
\text { Ellipsoidal }\end{array}$ & \\
\hline $\begin{array}{l}\text { Cryptococcus } \\
\text { macerans }\end{array}$ & T1S-1-PDA & Macrocystis pyrifera & $\begin{array}{l}\text { Bright } \\
\text { Pink }\end{array}$ & Shiny & Umbonate & 7.6 & $\begin{array}{l}\text { Elongate } \\
\text { Ellipsoidal }\end{array}$ & \\
\hline $\begin{array}{l}\text { Cryptococcus } \\
\text { sp. }\end{array}$ & Y2M-PDA & Codium convolutum & Peach & Glistening & & 5.4 & $\begin{array}{l}\text { Elongate } \\
\text { Ellipsoidal }\end{array}$ & \\
\hline $\begin{array}{l}\text { Cryptococcus } \\
\text { victoriae }\end{array}$ & L2S-1-PDA & Cystophora scalaris & Cream & Shiny & & 5.0 & Ellipsoidal & \\
\hline $\begin{array}{l}\text { Cryptococcus } \\
\text { victoriae }\end{array}$ & S4S-PDA & $\begin{array}{l}\text { Psaromenia } \\
\text { berggernii }\end{array}$ & Cream & Shiny & & 4.6 & Ellipsoidal & $\begin{array}{l}\text { Possible } \\
\text { Clustering }\end{array}$ \\
\hline $\begin{array}{l}\text { Cryptococcus } \\
\text { victoriae }\end{array}$ & $\begin{array}{l}\text { W5S-4- } \\
\text { STD }\end{array}$ & Pachymenia lusoria & Peach & Shiny & & 4.6 & $\begin{array}{l}\text { Elongate } \\
\text { Ellipsoidal }\end{array}$ & $\begin{array}{l}\text { Possible } \\
\text { Clustering }\end{array}$ \\
\hline
\end{tabular}




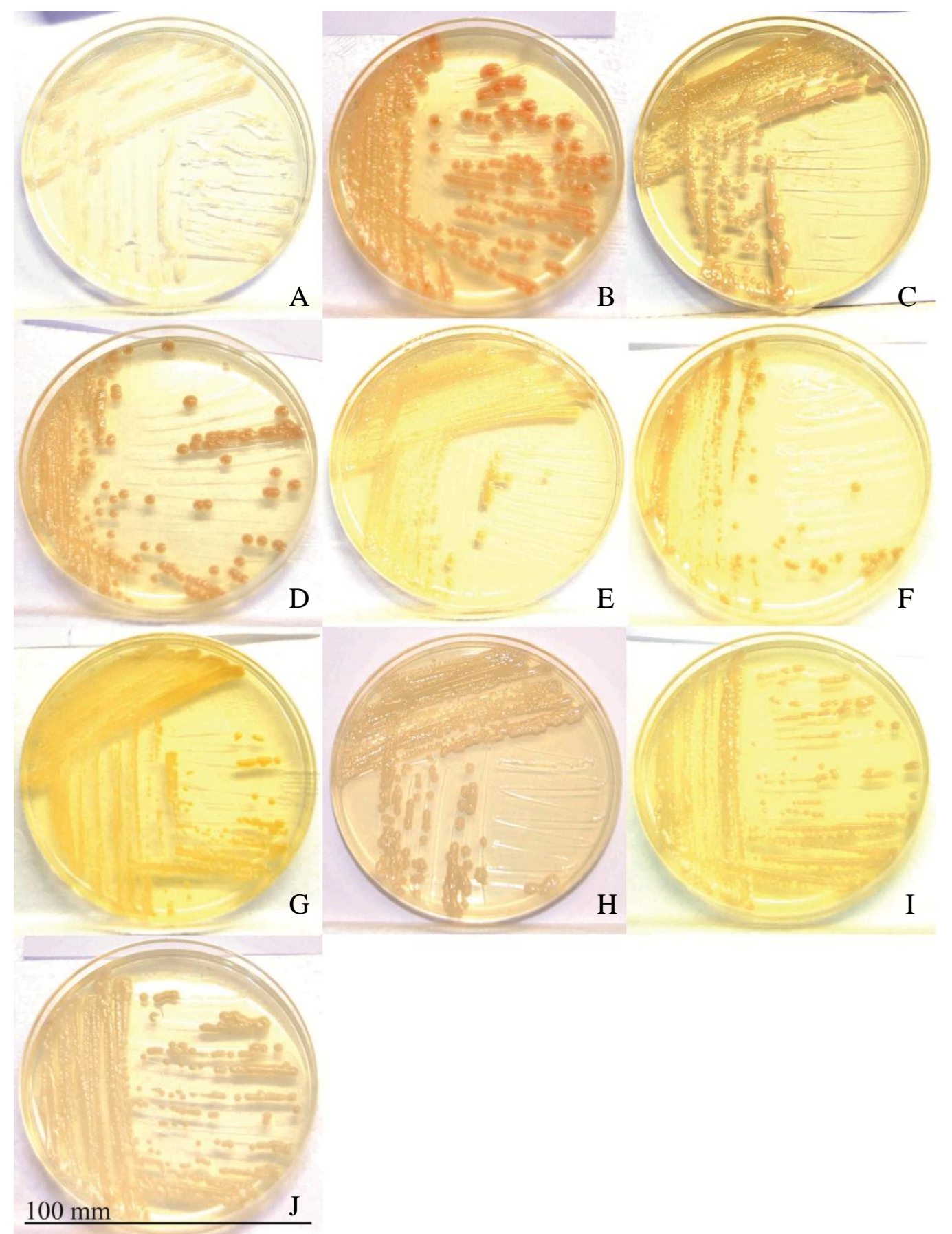

Figure 8. Photographs of colonies formed by Agaricomycotina isolates on agar plates. A=Z2SSTD (Cryptococcus carnescens), B=T1S-1-PDA (Cryptococcus macerans), C=Y2M-PDA (Cryptococcus sp.), D=T1S-3-PDA, E=Y5S-PDA, F=Z4S-1-STD (D, E, F all Cryptococcus dimennae), G=L2S-1-PDA, H=S4S-PDA, I=W5S-4-STD (G, H, I all Cryptococcus victoriae), $\mathrm{J}=\mathrm{V} 5 \mathrm{M}-\mathrm{PDA}$ (Bullera unica). 


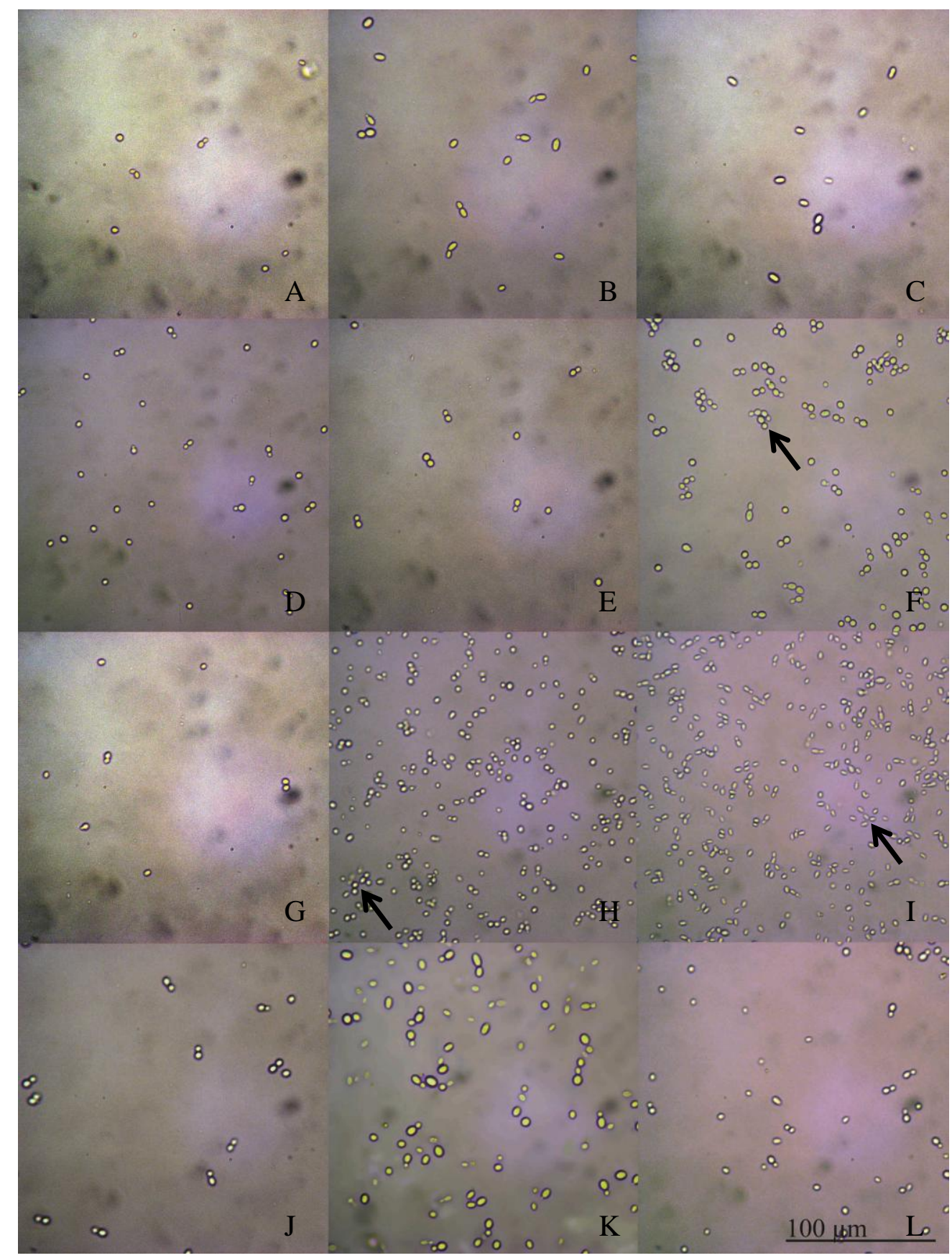

Figure 9. Microscopic images of Agaricomycotina isolates. A=Z2S-STD (Cryptococcus carnescens), B=T1S-1-PDA (Cryptococcus macerans), $\mathrm{C}=\mathrm{P} 4 \mathrm{M}-\mathrm{STD}$ (Cryptococcus macerans), D=T1S-3-PDA, E=Y5S-PDA, F=Z4S-1-STD (D, E, F all Cryptococcus dimennae), G=L2S-1PDA, H=S4S-PDA, I=W5S-4-STD (G, H, I all Cryptococcus victoriae), J=U3S-PDA (Cryptococcus chernovii), K=Y2M-PDA (Cryptococcus sp.), L=V5M-PDA (Bullera unica). 


\subsubsection{Pucciniomycotina}

\subsubsection{Identification via Molecular Techniques}

Twelve isolates were identified from the subphylum Pucciniomycotina (Table 2). These were comprised of seven isolates from the genus Rhodotorula and one isolate from each of the species Sakaguchia dacryoidea (Fell, I.L. Hunter \& Tallman) Y. Yamada, K. Maeda \& Mikata and Kondoa aeria Á. Fonseca, Samp. \& Fell. ITS sequences obtained from Pucciniomycotina isolates were of good quality and formed contigs ranging from 539 to 599 bp in length. The ITS contig from each isolate was queried against GenBank and gave excellent sequence identity (98 - 100 $\%)$ to a named Rhodotorula species, Rhodotorula sp., Kondoa aeria or Sakaguchia dacryoidea.

A phylogenetic tree was constructed with ITS sequences from Pucciniomycotina isolates plus selected sequences from GenBank and the literature (Fig. 10). Overall, the Pucciniomycotina phylogenetic tree separated ITS sequences from named species and Pucciniomycotina isolates into distinct clades with good fidelity. Sequences from uncultured fungi were also distributed within these clades and are not mentioned further as they did not contribute to isolate identification. One isolate (H10S-PDA) was situated in a well-supported clade with, and identified as, Kondoa aeria (bootstrap support $100 \%$ ). Due to their position in a well-supported clade with the species, three isolates (E5S-PDA, E3M-STD and Q5S-2-PDA) were identified as Rhodotorula mucilaginosa (bootstrap support $100 \%$ ). A single isolate (B4M-STD) was in a wellsupported clade with, and identified as, Rhodotorula minuta (Saito) F.C. Harrison (bootstrap 
support $100 \%$ ). Two isolates (J3S-STD and I1-STD) were identified as Rhodotorula slooffiae E.K. Novák \& Vörös-Felkai due to their position in a well-supported clade with this species (bootstrap support $92 \%$ ). One isolate (P2S-PDA) was situated in a well-supported clade with, and identified as, Rhodotorula sp. (bootstrap support $100 \%$ ). Due to its position in a wellsupported clade with the species, one isolate (Z4S-1-PDA) was identified as Sakaguchia dacryoidea (bootstrap support $82 \%$ ). Overall, the Pucciniomycotina phylogenetic tree displayed good resolution of isolates to a species level, with the exception of one isolate (P2S-PDA) which

was identified as Rhodotorula sp. Results from this phylogenetic tree were in agreement with those obtained from GenBank searches, and the combination of these two methods allowed confident identification of isolates from the Pucciniomycotina.

\subsubsection{Morphological Observations}

All isolates from the Pucciniomycotina had slightly raised or convex circular colonies with an entire margin (Fig. 11 and Table 6). Colonies ranged in colour from peach to bright pink and were shiny or glistening. The cell morphology of Pucciniomycotina isolates was variable; most isolates had an ellipsoidal cell shape, although a small number had elongated cells $(\mathrm{A}, \mathrm{B} \& \mathrm{H}$ in Fig. 12). A couple of isolates displayed what appeared to be a clustering of cells, but distinct chains of cells were not observed (as indicated in Fig. 12). Cell size ranged from 4.0 to $5.4 \mu \mathrm{m}$, with an average of $4.4 \mu \mathrm{m}$ (Table 6). Literary descriptions of yeasts from the Pucciniomycotina were generally in agreement with observations made during this investigation, thus, data from culture studies support the identification of these isolates (CBS-KNAW 2012; MycoBank 2012). 


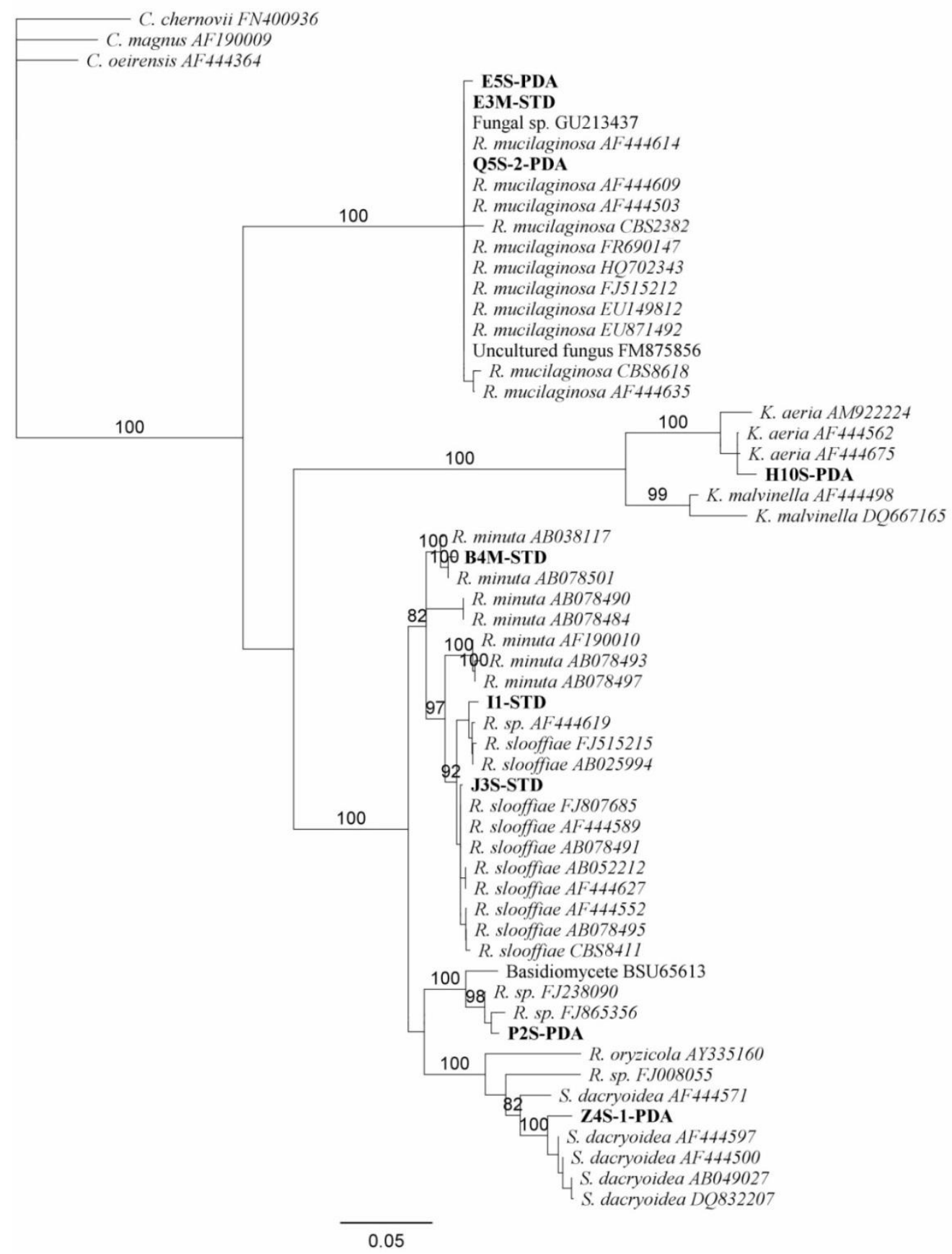

Figure 10. Neighbour-joining phylogenetic tree based on ITS sequences from Pucciniomycotina isolates (shown in bold), using the Tamura-Nei model. Bootstrap values are shown for nodes supported by $>70 \%$ of 100 replicates. Scale bar represents 0.05 substitutions per site. The outgroup contains ITS sequences from the genus Cryptococcus. 
Table 6. Morphology of Pucciniomycotina isolates.

\begin{tabular}{|c|c|c|c|c|c|c|c|}
\hline $\begin{array}{l}\text { Assigned } \\
\text { Organism }\end{array}$ & $\begin{array}{l}\text { Isolate } \\
\text { Code }\end{array}$ & Alga of Origin & $\begin{array}{l}\text { Colony } \\
\text { Colour }\end{array}$ & $\begin{array}{l}\text { Colony } \\
\text { Surface }\end{array}$ & $\begin{array}{c}\text { Average } \\
\text { Cell Size } \\
(\mu \mathrm{m})\end{array}$ & Cell Shape & $\begin{array}{l}\text { Additional } \\
\text { Notes: } \\
\text { Cellular } \\
\text { Appearance }\end{array}$ \\
\hline Kondoa aeria & H10S-PDA & Psaromenia berggernii & Peach & Glistening & 4.0 & $\begin{array}{l}\text { Elongate } \\
\text { Ellipsoidal }\end{array}$ & \\
\hline $\begin{array}{l}\text { Rhodotorula } \\
\text { minuta }\end{array}$ & B4M-STD & Pachymenia lusoria & Bright Pink & Shiny & 4.4 & Ellipsoidal & $\begin{array}{l}\text { Cluster } \\
\text { Formation }\end{array}$ \\
\hline $\begin{array}{l}\text { Rhodotorula } \\
\text { mucilaginosa }\end{array}$ & E3M-STD & Macrocystis pyrifera & Bright Pink & Shiny & 4.6 & Ellipsoidal & $\begin{array}{l}\text { Cluster } \\
\text { Formation }\end{array}$ \\
\hline $\begin{array}{l}\text { Rhodotorula } \\
\text { mucilaginosa }\end{array}$ & E5S-PDA & Macrocystis pyrifera & Bright Pink & Shiny & 4.2 & Ellipsoidal & $\begin{array}{l}\text { Possible } \\
\text { Clustering }\end{array}$ \\
\hline $\begin{array}{l}\text { Rhodotorula } \\
\text { mucilaginosa }\end{array}$ & Q5S-2-PDA & Cystophora scalaris & Bright Pink & Glistening & 4.2 & Ellipsoidal & \\
\hline $\begin{array}{l}\text { Rhodotorula } \\
\text { slooffiae }\end{array}$ & I1-STD & $\begin{array}{l}\text { Champia } \\
\text { novae-zelandiae }\end{array}$ & Bright Pink & Glistening & 4.0 & Ellipsoidal & $\begin{array}{l}\text { Possible } \\
\text { Clustering }\end{array}$ \\
\hline $\begin{array}{l}\text { Rhodotorula } \\
\text { slooffiae }\end{array}$ & J3S-STD & Codium convolutum & Peach & Glistening & 4.4 & $\begin{array}{l}\text { Elongate } \\
\text { Ellipsoidal }\end{array}$ & \\
\hline Rhodotorula sp. & P2S-PDA & Macrocystis pyrifera & Peach & Glistening & 4.8 & Ellipsoidal & \\
\hline $\begin{array}{l}\text { Sakaguchia } \\
\text { dacryoidea }\end{array}$ & Z4S-1-PDA & Caulerpa brownii & Bright Pink & Shiny & 5.4 & $\begin{array}{l}\text { Elongate } \\
\text { Ellipsoidal }\end{array}$ & $\begin{array}{l}\text { Possible } \\
\text { Clustering }\end{array}$ \\
\hline
\end{tabular}




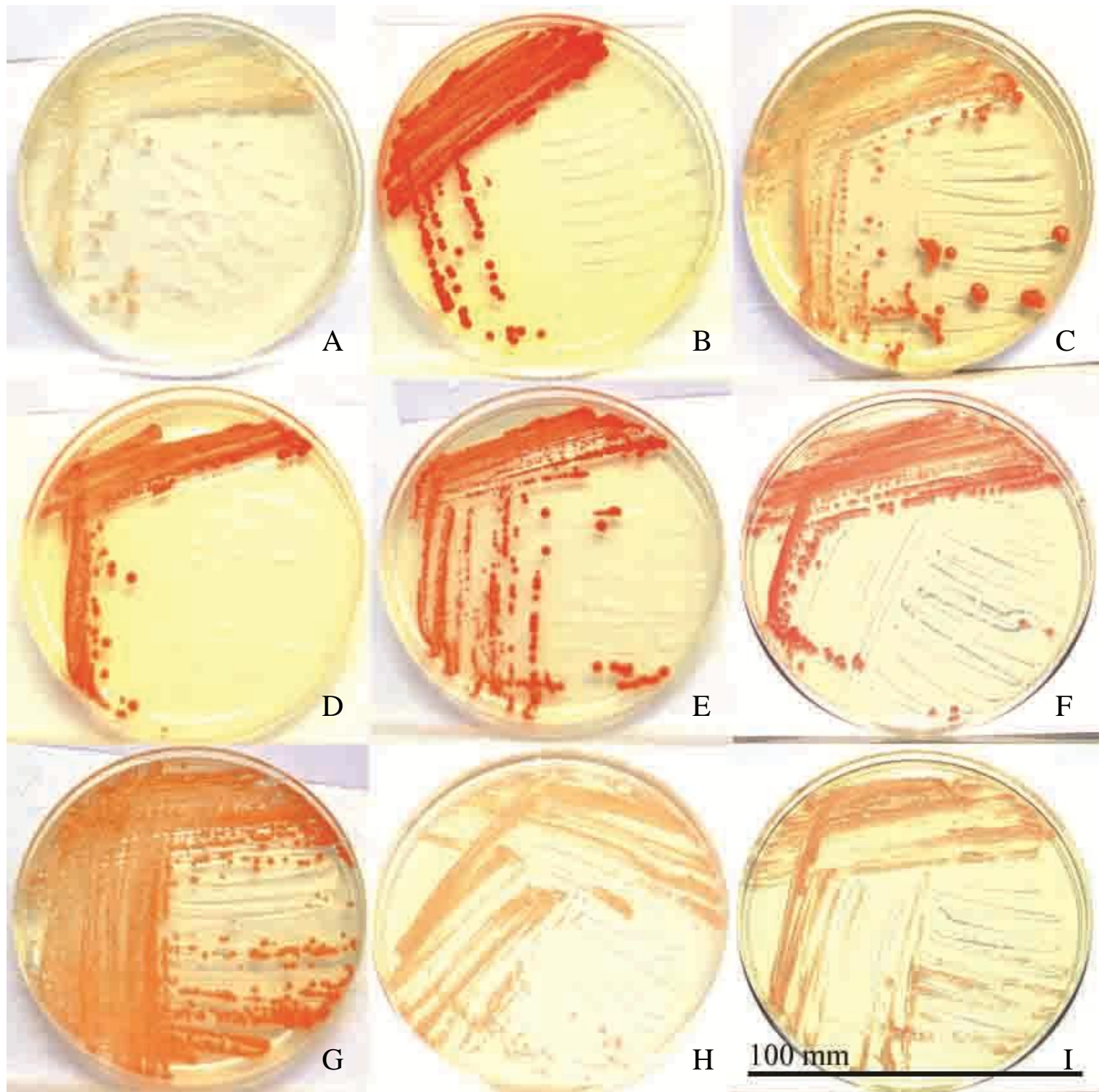

Figure 11. Photographs of colonies formed by Pucciniomycotina isolates on agar plates. A=H10S-PDA (Kondoa aeria), B=Z4S-1-PDA (Sakaguchia dacryoidea), C=B4M-STD (Rhodotorula minuta), D=E3M-STD, E=E5S-PDA, F=Q5S-2-PDA (D, E, F all Rhodotorula mucilaginosa), G=I1-STD (Rhodotorula slooffiae), H=J3S-STD (Rhodotorula slooffiae), I=P2SPDA (Rhodotorula sp.). 


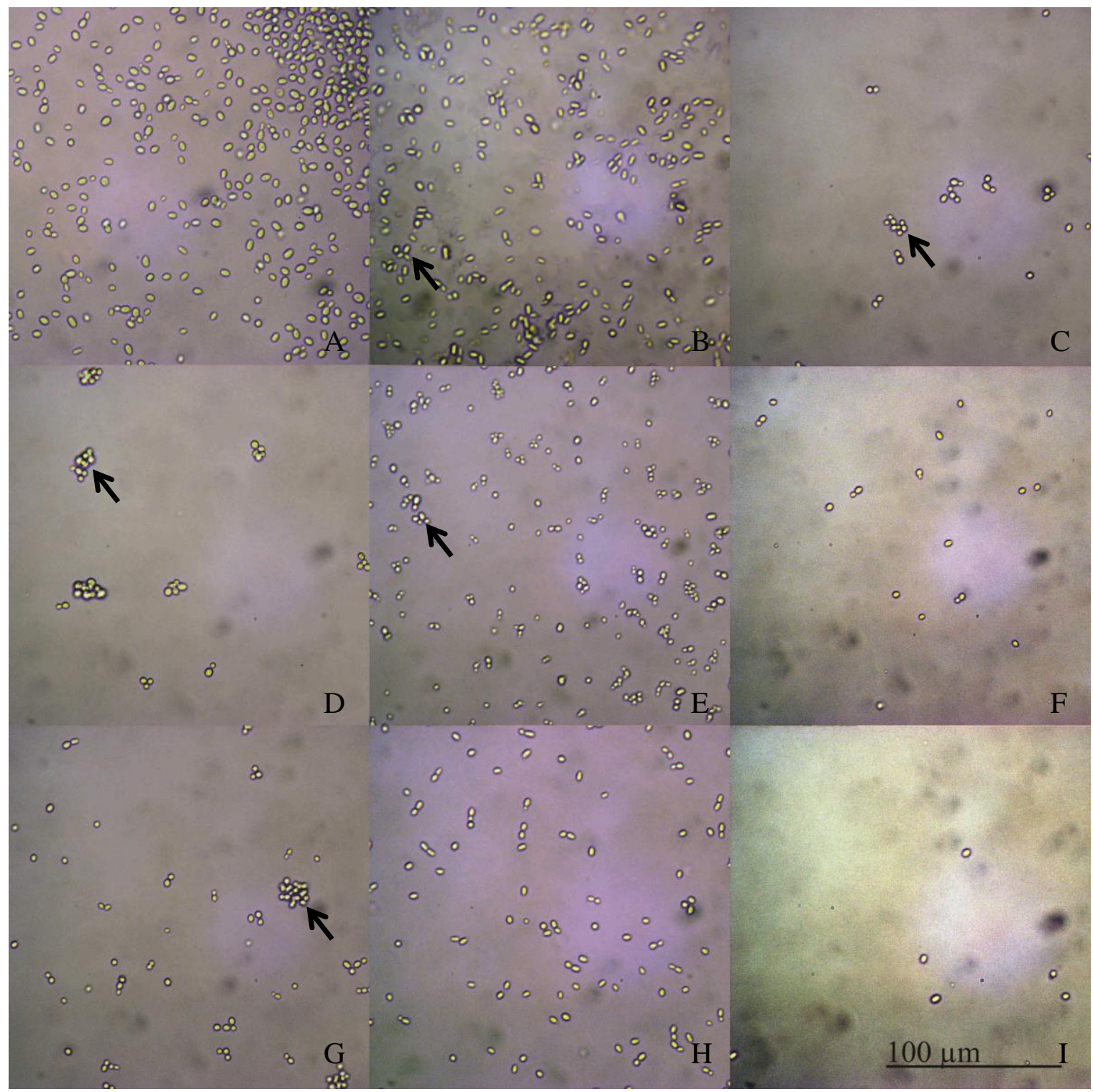

Figure 12. Microscopic images of Pucciniomycotina isolates. A=H10S-PDA (Kondoa aeria), B=Z4S-1-PDA (Sakaguchia dacryoidea), C=B4M-STD (Rhodotorula minuta), D=E3M-STD, E=E5S-PDA, F=Q5S-2-PDA (D, E, F all Rhodotorula mucilaginosa), G=I1-STD (Rhodotorula slooffiae), H=J3S-STD (Rhodotorula slooffiae), I=P2S-PDA (Rhodotorula sp.). 


\subsection{Isolate Distribution}

\subsubsection{Isolate Distribution by Subphylum}

\subsubsection{Pezizomycotina}

Yeasts identified as Aureobasidium pullulans were isolated on PDA (Table 7) from three algal species (Table 8) sampled during Collections 2 and 3 (Waitaha Cove) (Fig. 13). However, only three Aureobasidium pullulans isolates were obtained so no distribution patterns were apparent.

\subsubsection{Saccharomycotina}

Metschnikowia isolates did not demonstrate a preferential association with any particular media (Table 7) or alga (Table 8). Yeasts from the genus Metschnikowia occurred frequently and made up over half of the isolates obtained during Collections 2 and 3 (Waitaha Cove) (Fig. 13). Metschnikowia isolates made up less than a third of the yeasts obtained during Collections 1 (Tarakena Bay) and 4 (Onehunga Bay), however, it was unclear whether this represented a distribution pattern or was simply due to the low number of yeasts isolated during these collections. 


\subsubsection{Agaricomycotina}

No distribution trends could be determined for Bullera unica since only one isolate was obtained; V5M-PDA was isolated on PDA from Cladhymenia oblongifolia sampled during Collection 4 (Onehunga Bay) (Table 7, Table 8 and Fig. 13). Low numbers of Cryptococcus isolates were found on six of the algae sampled and did not demonstrate an obvious association with any particular alga (Table 8). In terms of media, Cryptococcus isolates were found more frequently on PDA (7) than STD (4) (Table 7). The number of Cryptococcus isolates obtained was variable between collections; no Cryptococcus isolates were found during Collection 1 (Tarakena Bay), a small number were obtained during Collections 2 and 3 (Waitaha Cove), and Cryptococcus made up $42 \%$ of the yeasts isolated during Collection 4 (Onehunga Bay) (Fig. 13). However, it was unclear whether variation in the number of Cryptococcus isolates obtained between collections was representative of a distribution pattern, since only 11 isolates were obtained from this genus.

\subsubsection{Pucciniomycotina}

No distribution trends could be determined for Sakaguchia dacryoidea and Kondoa aeria, since only one isolate was obtained from each species (Table 7, Table 8 and Fig. 13). Kondoa aeria was isolated on PDA from Psaromenia berggernii during Collection 2 (Waitaha Cove), and Sakaguchia dacryoidea was isolated on PDA from Caulerpa brownii during Collection 4 (Onehunga Bay). Rhodotorula isolates did not demonstrate a clear preference for any particular media (Table 7). Furthermore, it was unclear whether Rhodotorula isolates demonstrated an 
association with any particular alga, since isolates were found on Champia novae-zelandiae, Codium convolutum, Cystophora scalaris, Pachymenia lusoria and Macrocystis pyrifera (Table 8). The number of Rhodotorula isolates obtained was highly variable between collections; Rhodotorula made up half the isolates from Collection 1 (Tarakena Bay), a small proportion of the isolates from Collections 2 and 3 (Waitaha Cove), and was not detected during Collection 4 (Onehunga Bay) (Fig. 13). However, only seven Rhodotorula isolates were obtained during this investigation, an inadequate number to reveal any distribution patterns.

3.5.2 Possible Influences on Isolate Biodiversity

\subsubsection{Isolation Media}

Out of 94 successfully isolated yeasts, 52 were found on PDA and 42 on STD media (Table 7). The isolation frequency of yeasts from the genera Metschnikowia, Cryptococcus and Rhodotorula was similar on both STD and PDA. Yeasts from the genera Aureobasidium, Bullera, Kondoa and Sakaguchia were only isolated on PDA, although they occurred infrequently. 


\subsubsection{Algal Host}

Ten different algal species were sampled during four collections (Table 8). Champia novaezelandiae, Cladhymenia oblongifolia and Pachymenia lusoria were sampled during two collections, whereas Ulva sp. and Carpophyllum maschalocarpum were only sampled during Collection 1. For the purposes of detecting any trends, algae sampled during three or all four collections were considered: Macrocystis pyrifera, Cystophora scalaris, Caulerpa brownii, Codium convolutum and Psaromenia berggernii. Champia novae-zelandiae was also considered as it yielded 12 isolates despite being sampled in just two collections. On average, 4.8 yeasts were isolated from each of the six algae considered per collection, and no alga yielded considerably fewer or more isolates. Fifty isolates were obtained from the genus Metschnikowia and the number isolated from each algal species was reasonably constant. The eleven Cryptococcus isolates were equally distributed between all algal species considered except for Champia novae-zelandiae, and the seven Rhodotorula isolates were equally distributed between Macrocystis pyrifera, Cystophora scalaris, Codium convolutum and Champia novae-zelandiae. Caulerpa brownii, Cystophora scalaris and Macrocystis pyrifera each yielded a single Aureobasidium pullulans isolate. The single Sakaguchia dacryoidea isolate was found on Caulerpa brownii, and the Kondoa aeria isolate was found on Psaromenia berggernii. Yeasts were also isolated from infrequently sampled algae; Cladhymenia oblongifolia yielded a Bullera unica isolate plus four unidentified yeasts, Pachymenia lusoria yielded one isolate each from Cryptococcus and Rhodotorula, and Ulva sp. yielded one Metschnikowia isolate. Although data were insufficient to draw conclusions regarding infrequently sampled yeasts or algae, yeasts from frequently isolated genera did not demonstrate a clear preference for any particular alga. 


\subsubsection{Location}

Differences were observed in the types of yeasts isolated between algal collections (Fig. 13). Around half of the yeasts isolated during Collection 1 (Tarakena Bay on 18/12/09, sea surface temperature of $14.8^{\circ} \mathrm{C}$ ) were from Rhodotorula, a third were from Metschnikowia and the rest were unidentified. During Collection 2 (Waitaha Cove on 29/01/10, sea surface temperature of 14 ${ }^{\circ} \mathrm{C}$ ), the majority of the isolates were from Metschnikowia, a quarter were unidentified, and one

or two yeasts were isolated from Rhodotorula, Cryptococcus, Kondoa and Aureobasidium. Despite the higher temperature, the yeast species isolated during Collection 3 (Waitaha Cove on 25/02/10, sea surface temperature of $17.9^{\circ} \mathrm{C}$ ) were very similar to those from Collection 2: the majority of the isolates were from Metschnikowia, $16 \%$ were unidentified, and one or two yeasts were isolated from Rhodotorula, Aureobasidium and Cryptococcus. During Collection 4 (Onehunga Bay on 19/03/10, sea surface temperature of $16.2{ }^{\circ} \mathrm{C}$ ), $42 \%$ of the yeasts isolated were from Cryptococcus, around one quarter were unidentified, less than one quarter were from Metschnikowia, and one yeast was isolated from each of the following genera: Bullera and Sakaguchia . 
Table 7. Isolation frequency of different yeast genera on two types of media.

\begin{tabular}{|l|l|l|}
\hline \multicolumn{1}{|c|}{ Yeast Genus } & \multicolumn{1}{|c|}{$\begin{array}{c}\text { Number of Colonies on Difco } \\
\text { Potato Dextrose Agar Plus (PDA) }\end{array}$} & $\begin{array}{c}\text { Number of Colonies on Difco } \\
\text { Standard Marine Agar Plus (STD) }\end{array}$ \\
\hline Aureobasidium & 3 & 0 \\
\hline Metschnikowia & 24 & 26 \\
\hline Bullera & 1 & 0 \\
\hline Cryptococcus & 7 & 4 \\
\hline Kondoa & 1 & 0 \\
\hline Sakaguchia & 1 & 0 \\
\hline Rhodotorula & 3 & 4 \\
\hline Unidentified & 12 & 8 \\
\hline Total & $\mathbf{5 2}$ & $\mathbf{4 2}$ \\
\hline
\end{tabular}


Table 8. Isolation frequency of different yeast genera from ten algal species.

\begin{tabular}{|c|c|c|c|c|c|c|c|c|c|c|}
\hline Yeast Genus & $\begin{array}{c}0 \\
0 \\
0 \\
0 \\
0 \\
0 \\
0 \\
0 \\
0 \\
0 \\
0 \\
0\end{array}$ & 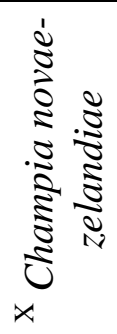 & 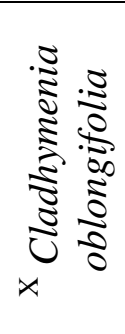 & 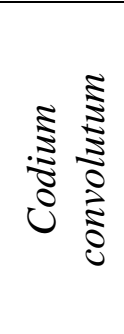 & 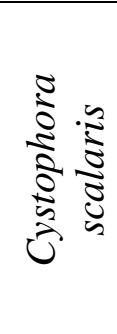 & 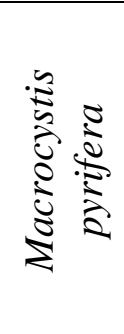 & 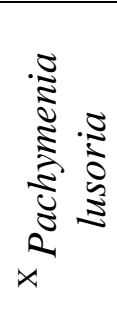 & 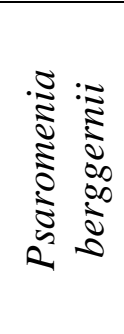 & 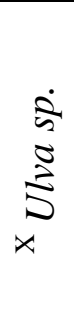 & 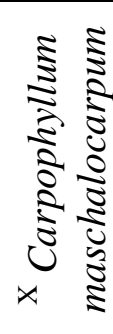 \\
\hline Aureobasidium & 1 & 0 & 0 & 0 & 1 & 1 & 0 & 0 & 0 & 0 \\
\hline Metschnikowia & 7 & 9 & 0 & 12 & 9 & 4 & 0 & 8 & 1 & 0 \\
\hline Bullera & 0 & 0 & 1 & 0 & 0 & 0 & 0 & 0 & 0 & 0 \\
\hline Cryptococcus & 2 & 0 & 0 & 2 & 2 & 3 & 1 & 1 & 0 & 0 \\
\hline Kondoa & 0 & 0 & 0 & 0 & 0 & 0 & 0 & 1 & 0 & 0 \\
\hline Sakaguchia & 1 & 0 & 0 & 0 & 0 & 0 & 0 & 0 & 0 & 0 \\
\hline Rhodotorula & 0 & 1 & 0 & 1 & 1 & 3 & 1 & 0 & 0 & 0 \\
\hline Unidentified & 3 & 2 & 4 & 1 & 5 & 4 & 0 & 1 & 0 & 0 \\
\hline Total & 14 & 12 & 5 & 16 & 18 & 15 & 2 & 11 & 1 & $\mathbf{0}$ \\
\hline
\end{tabular}

$\mathrm{X}$ denotes algae sampled during only one or two collections. 


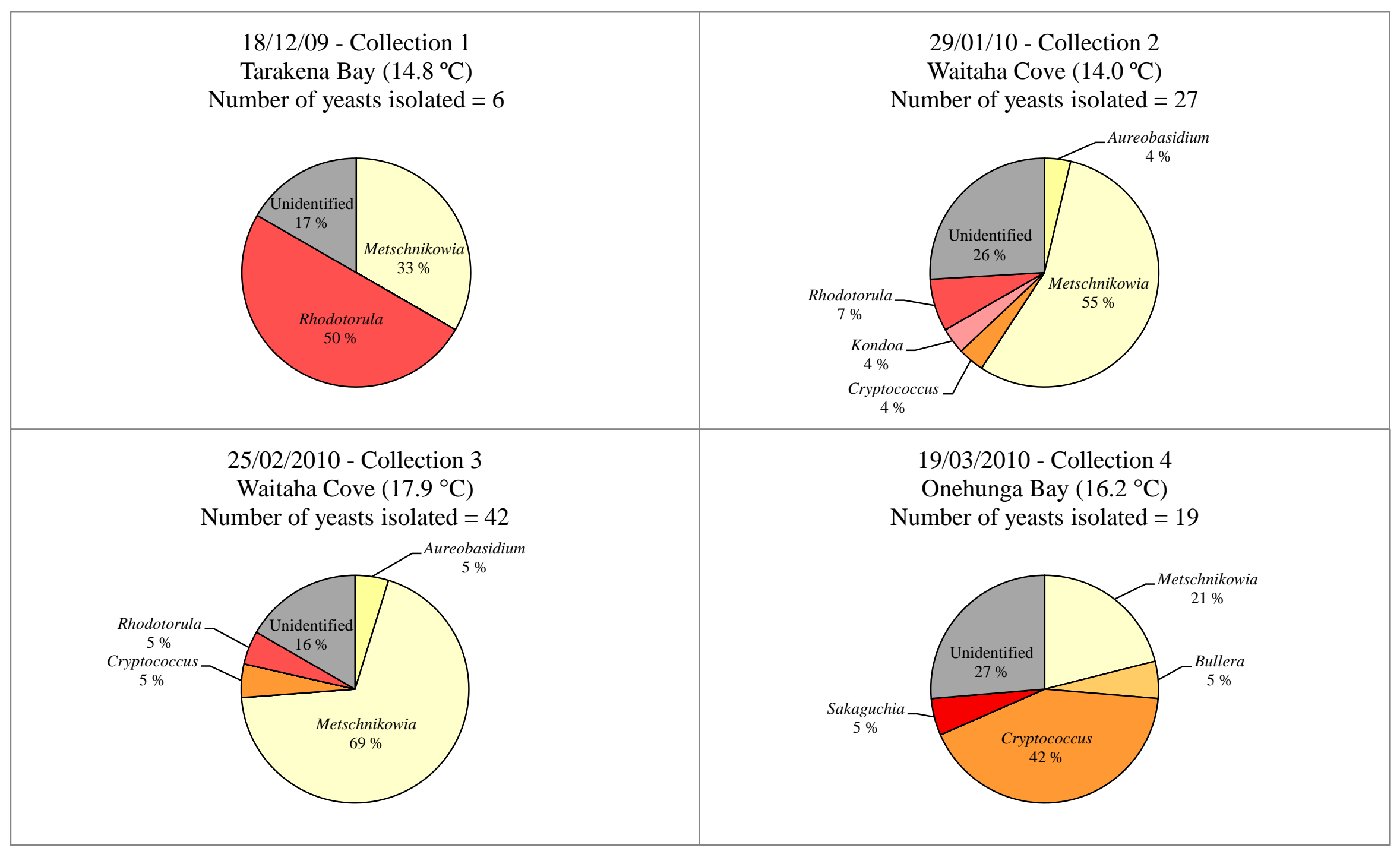

Figure 13. Pie graphs illustrating the isolation frequency of different yeast genera during four algal collections. 


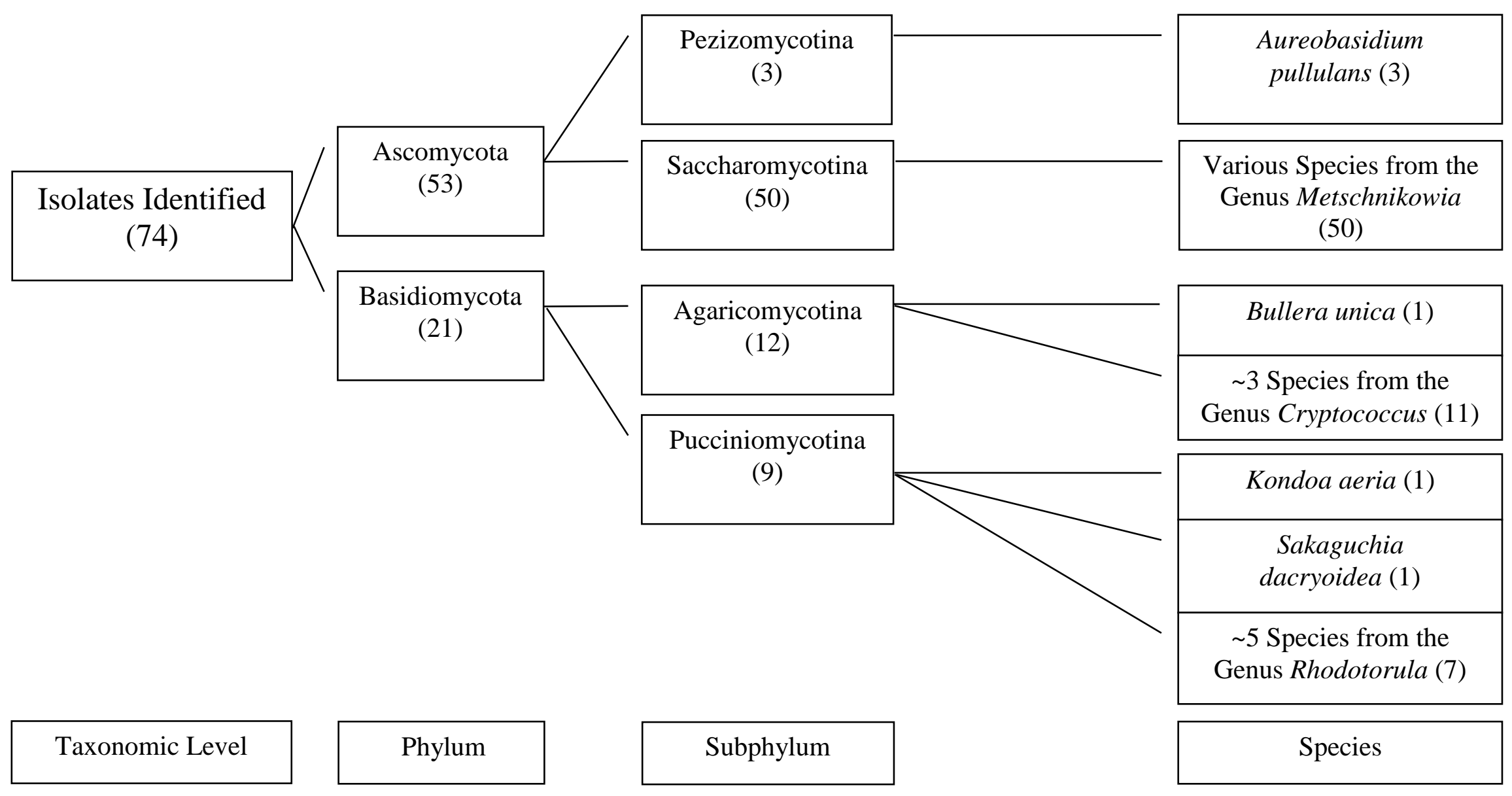

Figure 14. Summary of isolates identified at three taxonomic levels. The number of isolates in each category is given in brackets. 


\subsection{Summary of Results}

During this investigation, 106 yeasts were selected from original inoculation plates, 94 of which were successfully isolated and found to be pure. ITS PCR products from 86 isolates were sent away for sequencing and 74 of these were of sufficient quality for yeast identification. ITS sequence data allowed identification via phylogenetic analysis and GenBank searches. Data regarding colony morphology and the microscopic appearance of cells generally confirmed isolate identity. Out of 74 yeasts identified, 53 isolates were from the Ascomycota and 21 were from the Basidiomycota. Yeasts identified from the Ascomycota were from two subphyla: 50 isolates were identified from the Saccharomycotina and three isolates were from the Pezizomycotina. All three isolates from the Pezizomycotina were confidently identified as the yeast-like fungus Aureobasidium pullulans. All 50 isolates from the Saccharomycotina were identified from the genus Metschnikowia: two isolates were identified as Metschnikowia zobellii but the remaining 48 isolates could not be identified further than the genus level. Isolates from the Basidiomycota were also from two subphyla: 12 isolates were identified from the Agaricomycotina and nine isolates were from the Pucciniomycotina. Yeasts obtained from the Agaricomycotina consisted of a single Bullera unica isolate plus 11 isolates from the genus Cryptococcus (three isolates each from Cryptococcus dimennae and Cryptococcus victoriae, two from Cryptococcus macerans, one each from Cryptococcus carnescens and Cryptococcus chernovii, and an unresolved Cryptococcus sp.). Yeasts obtained from the Pucciniomycotina consisted of single isolates from Sakaguchia dacryoidea and Kondoa aeria plus seven isolates from the genus Rhodotorula (three isolates from Rhodotorula mucilaginosa, two from Rhodotorula slooffiae, one from Rhodotorula minuta and an unresolved Rhodotorula sp.). 


\section{Discussion}

\subsection{Observations}

There is currently debate as to whether microorganisms are ubiquitous or if they exhibit biogeographic patterns (Martiny et al. 2006). To address these questions of biodiversity, results from this investigation are discussed in light of evidence from the literature. Yeasts isolated during this investigation were from similar genera and demonstrated high ITS sequence similarity with yeasts from diverse substrates and environments worldwide. Similarity observed between the isolates obtained and yeasts from other sources may be suggestive of marine yeast ubiquity at a global level, or as proposed by Baas Becking and Beijerinck's widely accepted tenet, "everything is everywhere, but, the environment selects" (Baas Becking 1934; De Wit and Bouvier 2006).

Although this was the first known investigation of marine yeast biodiversity from waters surrounding New Zealand's main Islands, the species isolated were not unique. Ninety-four yeasts were isolated from algae sampled in the Wellington Region, 74 of which were identified from a variety of previously recognised species in seven genera and four subphyla (Fig. 14). Most isolates belonged to Metschnikowia, Rhodotorula or Cryptococcus, a similar range of genera to those detected from diverse habitats worldwide. Candida, Cryptococcus, Rhodotorula and Debaryomyces are regularly detected from various marine environments (Kutty and Philip 2008, plus references therein), such as the deep sea of the Pacific Ocean (Yamasato et al. 1974; 
Nagahama 2006) and the fresh, estuarine and marine waters of Florida (Ahearn et al. 1968). Many yeasts isolated during this investigation also demonstrated high identity to previously documented ITS sequences (Table 2); the high level of ITS sequence identity indicates similarity between the isolates obtained and yeasts from diverse marine and terrestrial substrates worldwide. For example, one isolate identified as Rhodotorula mucilaginosa (E3M-STD) had $100 \%$ identity to ITS sequences obtained from New Zealand oak trees (unpublished sequence in GenBank, submitted by H. Zhang, R. C. Gardner, A. Skelton and M. R. Goddard) and seawater from the coast of Taiwan (unpublished sequence in GenBank, submitted by C. F. Chang and S. M. Liu). One isolate identified as Cryptococcus carnescens (Z2S-STD) had 99 - $100 \%$ identity to ITS sequences from a range of different sources: grapevines (Al Rwahnih et al. 2011), indoor dust (Pitkaranta et al. 2008), plus soils and historic wood from the Ross Sea Region of Antarctica (Arenz et al. 2006).

Yeasts obtained during this investigation also demonstrated high ITS sequence similarity with strains originally isolated from the Chatham Islands. Twelve isolates of Candida natalensis and a single Metschnikowia zobellii isolate were collected from tidal pools on the Chatham Islands, and six further Metschnikowia zobellii isolates were found in a small freshwater lake (Fell and Hunter 1968). Although originally identified by morphological features as Metschnikowia zobellii, further morphological investigation and results from mating tests indicated that some isolates from the Chatham Islands should be redesignated as Metschnikowia bicuspidata var. chathamia (Fell and Pitt 1969). Original type strains of Metschnikowia bicuspidata var. chathamia from the Chatham Islands now have ITS sequence data available (CBS-KNAW 2012), and some demonstrated high ITS sequence similarity with isolates obtained during this investigation. An 
unsupported group in the Saccharomycotina phylogenetic tree contained ITS sequences from strains of Metschnikowia bicuspidata var. chathamia and yeasts isolated during this investigation (Fig. 4); 25 individual isolates with identical partial ITS sequences shared a pairwise identity of $99.1 \%$ with strain CBS5980 and a pairwise identity of $99.5 \%$ with strain CBS5903.

Results from this investigation and limited evidence from the literature suggest that marine yeasts are ubiquitous at a global level, or as proposed by Baas Becking and Beijerinck's widely accepted tenet, "everything is everywhere, but the environment selects" (Baas Becking 1934; De Wit and Bouvier 2006). The distribution of marine yeasts is recognised as widespread (Van Uden 1960) and they have been detected from various substrates: seawater, sediments, algae, vertebrates and invertebrates (Roth et al. 1962; Kohlmeyer and Kohlmeyer 1979). Marine yeasts have also been observed in diverse habitats including tropical (Zinjarde and Pant 2002), Antarctic (Vishniac 2006) and extreme environments (Raspor and Zupan 2006), the deep sea (Yamasato et al. 1974; Nagahama 2006), and now from waters surrounding New Zealand's main Islands. The South Pacific Ocean has not been extensively sampled for marine yeasts (Kutty and Philip 2008) so the results of this investigation provide a valuable source of information. Data collected during this investigation did not reveal any biogeographic patterns or novel species; the isolates obtained were similar to previously recognised yeasts. Most marine yeasts isolated during this investigation belonged to Metschnikowia, Rhodotorula or Cryptococcus, a comparable range of genera to those detected from diverse habitats worldwide. Many isolates also demonstrated high ITS sequence similarity with yeasts from distant environments, or isolates obtained from the Chatham Islands in the 1960's (Fell and Hunter 1968). The high level of similarity observed, despite the large geographic distances between yeasts detected during this and other 
investigations, may reflect a widespread distribution of yeasts at a global level. High sequence similarity between yeasts from diverse sources and the isolates obtained during an investigation has been recorded previously (Singh et al. 2010), and may contribute to evidence which suggests marine yeast ubiquity. Furthermore, 20 types of filamentous marine fungi that were previously known from the Northern Hemisphere have been detected in New Zealand, suggesting that New Zealand's marine mycoflora is similar to that of comparable regions (Lintott and Lintott 1982).

The seemingly ubiquitous distribution of marine yeasts from diverse substrates and locations worldwide may be due to their ease of dispersal and adaptable nature, under the influence of existing environmental conditions. The small size and huge abundance of microorganisms may facilitate their dispersal over large distances by essentially random forces (Finlay and Esteban 2001; Finlay 2002) and result in the introduction of microorganisms to new locations (Martiny et al. 2006). For example, it has been suggested that frequent dispersal occurs between terrestrial and deep sea habitats (Bass et al. 2007); waterlogged wood and particulate organic matter from the sea surface may sink, contributing species to the deep sea (Singh et al. 2010, plus references therein). The similarities observed between yeasts isolated during this investigation and those from elsewhere may be partially due to simple dispersal processes such as ocean currents, which appear to move from the main Islands of New Zealand towards the Chatham Islands (Heath 1985). It has been suggested that the common presence of filamentous marine fungi Halosphaeria mediosetigera Cribb \& J.W. Cribb and Ceriosporopsis halima Linder in Queensland and New Zealand waters is in agreement with the flow of warm ocean currents from Queensland to New Zealand (Lintott and Lintott 1982). The adaptable nature of marine yeasts may also contribute to their ubiquitous distribution; fungi are known to be ecologically successful 
and occupy a wide range of niches due to their highly versatile physiological adaptations (Nagano et al. 2010). Marine fungi are generally recognised as an adaptable group (Kohlmeyer 1963), and yeast cells have mechanisms that allow them to withstand the diverse range of stress inducing conditions to which they are continuously exposed (Tanghe et al. 2006). For example, halophilic marine yeasts such as Debaryomyces hansenii are often found in shallow seawater and other salty environments (Raspor and Zupan 2006, plus references therein). Certain yeasts are even known as extremophiles due to their ability to thrive under various extreme conditions (Raspor and Zupan 2006). This ease of microbial dispersal and their subsequent colonisation of new environments may lead to increased gene flow and overcome any tendency towards genetic and spatial differentiation (Martiny et al. 2006). Despite their generally adaptable nature, existing environmental conditions such as temperature, light, pressure, $\mathrm{pH}$, water and nutrient availability are known to influence yeast populations (Dix and Webster 1995; Deák 2006). Pollution, terrestrial runoff and the availability and concentration of organic material may also modify the character of yeast populations in coastal waters (Fell and Van Uden 1963; Kohlmeyer and Kohlmeyer 1979; Hagler and Mendonça-Hagler 1981). The selective survival of certain marine yeasts in various aquatic environments has been suggested (Ahearn et al. 1968). Existing environmental conditions may act upon a large reservoir or seed bank of microbial species in a given environment, allowing certain species to thrive while others persist only as spores or cysts until suitable conditions arise (Finlay and Esteban 2001; Fenchel and Finlay 2004). One suggestion is that microorganisms are generally ubiquitous, and will grow wherever their preferred habitat is realised (Boenigk et al. 2006, plus references therein). Yeast genera such as Cryptococcus could contribute to a dormant marine yeast seed bank because they produce chlamydospores and very thick capsules, which protect against and ensure survival in extreme 
environments (Raspor and Zupan 2006, plus references therein). Yeasts have also been detected from piles of decomposing kelp, which may act as a reservoir of microbial populations that are released when the kelp is washed back into the sea (Van Uden and Castelo Branco 1963).

In contrast to previously mentioned evidence, certain research supports the idea that free-living microorganisms demonstrate biogeographic distribution patterns, and that past events or geographic barriers may be responsible for shaping spatial variation in microorganism biodiversity (Boenigk et al. 2006; Martiny et al. 2006). One suggestion is that most microbial eukaryotes are ubiquitous, although the possibility of subtle cases of endemism is recognised (Finlay and Esteban 2001; Boenigk et al. 2006). For example, geographic isolation was found to be primarily responsible for the global population structure of the archaeon Sulfolobus, which demonstrated clustering by geographic locale rather than current environmental conditions (Whitaker et al. 2003). It was suggested that gene flow between Sulfolobus populations is restricted over large distances, and that the highly specialized growth requirements of Sulfolobus limit their introduction and persistence in new locations with established microorganism populations (Whitaker et al. 2003). Marine yeasts appear to be widespread (Kutty and Philip 2008, plus references therein), but further investigation might reveal biogeographic distribution patterns at a local level. Certain oceanic yeasts are thought to demonstrate ubiquity or occur by chance, although other rare yeasts are restricted biologically and geographically (Fell 1976). If the distribution of marine yeasts is restricted, it could be due to their nutritional requirements or saprophytic or parasitic dependence on a specific group of marine plants or animals (Fell 1976). 


\subsection{Limited Isolate Identification}

Although they were the primary source of information used for isolate identification, ITS sequences did not enable confident identification of all isolates obtained during this investigation. Yeasts are frequently identified based on phylogenetic construction with rRNA sequence data (Valente et al. 1999; Nagahama et al. 2001; Kurtzman and Robnett 2003; Singh et al. 2010), and high ITS sequence identity $(97-100 \%)$ between yeasts isolated during this investigation and named yeast sequences available in GenBank enabled identification of many isolates (Table 2). However, 48 Metschnikowia isolates could not be confidently identified further than the genus level. The identification difficulties demonstrated by Metschnikowia isolates may be because their ITS sequences were of insufficient quality for phylogenetic analysis; ITS sequences from some Metschnikowia isolates displayed several undetermined nucleotides, which often occurred at the same positions within this gene region. The frequent occurrence of poor quality ITS sequences among Metschnikowia isolates may be due to low quantity PCR products, or a technical problem such as sequence primer mismatch during the PCR reaction, although no mention of this is apparent in the literature. Metschnikowia sequences that were of sufficient quality for contig construction and use in phylogenetic analysis also had limitations (Fig. 4); poor separation and support of branches in the Saccharomycotina phylogenetic tree meant that phylogenetic data were unable to confirm isolate identifications based on GenBank search results. Poor branch separation may be due to the high level of ITS sequence similarity among previously recognised yeasts from the genus Metschnikowia and isolates obtained during this investigation. For example, 25 individual isolates with identical partial ITS sequences (represented by $\mathrm{X}$ in the Saccharomycotina phylogenetic tree) shared a pairwise identity of up to $99.5 \%$ with ITS 
sequences from Metschnikowia bicuspidata (U51436), Metschnikowia bicuspidata var. bicuspidata (CBS5575) and Metschnikowia bicuspidata var. chathamia (CBS5903). Metschnikowia bicuspidata, Metschnikowia zobellii and Metschnikowia australis (Fell \& I.L. Hunter) Mend.-Hagler, Hagler, Phaff \& Tredick are closely related, as demonstrated by their production of asci in cross matings (Fell and Hunter 1968) and by the grouping of these species in a consensus tree constructed with LSU sequence data (Nguyen et al. 2006). Metschnikowia bicuspidata var. bicuspidata and Metschnikowia australis appear to be more closely related to each other than to Metschnikowia zobellii, despite an intermediate level of base sequence complementarity in DNA-DNA reassociation experiments indicating some genomic divergence between the former two species (Mendonça-Hagler et al. 1985). Analysis of additional rRNA regions that demonstrate sequence divergence, such as the LSU or SSU, might enable further resolution of isolate identity (Mendonça-Hagler et al. 1993; Kurtzman and Fell 2006).

Morphological and physiological descriptions are frequently used to support initial isolate identifications based on rRNA sequence data (Nagahama et al. 2001; Singh et al. 2010). The primary identification of yeasts isolated during this investigation was based on ITS sequence data and typically supported by morphological observations (Tables 3-6); morphological features of yeast isolates were generally in agreement with descriptions of corresponding yeast type strains from the databases MycoBank and CBS-KNAW (CBS-KNAW 2012; MycoBank 2012). For example, isolate B4M-STD was identified as Rhodotorula minuta based on ITS sequence data, and descriptions which indicate that this species has pink coloured colonies support this identification (CBS-KNAW 2012). However, the subjective nature of describing yeast cell morphology at a microscopic level limited the extent to which morphological observations were 
able to support the identification of isolates. For example, difficulties were experienced when determining whether the shape of a cell should be described as ellipsoidal or an elongated ellipsoid, or whether the chains or clusters of cells displayed by isolates such as Cryptococcus dimennae and Cryptococcus victoriae (as indicated in Fig. 9) were indicative of cells which did not detach after budding, cells clustered together via sedimentation, or possibly the formation of pseudohyphae. Certain strains of yeast are known to produce pseudohyphae (CBS-KNAW 2012; MycoBank 2012), but clear pseudohyphae formation was not observed during microscopic analysis in this investigation. The use of morphological and physiological observations to support isolate identification has further limitations; results from morphological and physiological analysis may be ambiguous due to strain variability (Kurtzman and Robnett 1998) and can demonstrate variable results both between and within species (Fell et al. 2000). Although there were no obvious contradictions, slight discrepancies occurred between morphological descriptions from the literature and the morphology demonstrated by some isolates. For example, isolates identified as Rhodotorula mucilaginosa had an ellipsoidal cell shape, although yeasts from the genus Rhodotorula demonstrate natural variation in cell morphology (Hasegawa et al. 1960). It is recommended that isolate identification be based on more than two techniques (Singh et al. 2010), and further identification of isolates might be possible via analysis of additional rRNA regions, morphological or physiological characteristics. Although recognised as a physiologically homogenous genus (Giménez-Jurado et al. 1995) more accurate identification of Metschnikowia isolates might be achieved via analysis of certain characteristics, such as biochemical properties (Mendonça-Hagler et al. 1985), maximum growth temperature, ascus length (Fell and Hunter 1968) and the number of ascospores produced per ascus (Fell and Pitt 1969). 


\subsection{Limited Representation of Biodiversity}

Due to limitations of the collection procedures used, this investigation is likely to be only partially representative of marine yeast biodiversity in New Zealand. In terms of yeast isolation from algal samples, the experimental procedures used were in accordance with other studies (Van Uden and Castelo Branco 1963; Seshadri and Sieburth 1971; Kohlmeyer and Demoulin 1981). However, this was a preliminary investigation; a limited number of algae were sampled during four collections conducted in the Wellington Region and only 94 yeasts were isolated in total (Fig. 13). Accordingly, the amount of data obtained with regards to marine yeast populations was limited and the results of this investigation are likely to represent just some of New Zealand's marine yeast biodiversity. Data limitations also hindered the development of conclusions as to whether experimental procedures, the type of alga sampled, or other environmental factors were influential on the yeasts isolated; slight differences were observed in the type and frequency of yeasts isolated between algal collections, but the significance of these differences was unclear. The number of new species detected generally decreases each time an area is sampled (Fenchel and Finlay 2004; Lachance 2006), but more extensive sampling would still be expected to reveal further marine yeast species in New Zealand. Sampling additional algae, substrates, locations, and during different seasons might allow a more accurate representation of New Zealand's marine yeast biodiversity, and possibly reveal biogeographic distribution patterns.

Limitations of culture techniques used to isolate yeasts from algae mean that the isolates obtained during this investigation only represent some of New Zealand's marine yeast biodiversity. Yeast isolation procedures were similar to those from other investigations in terms of the culture 
techniques and isolation media used (Nagahama et al. 2001; Nagahama 2006). However, yeast isolation techniques have limitations for capturing biodiversity; a range of isolates were obtained using culture-based detection methods, but standard culture techniques are unable to detect "nonculturable" yeasts (Boundy-Mills 2006). It is unclear how much biodiversity has been overlooked, since the numbers of non-viable and nonculturable yeasts in marine environments are unknown (Fell 2001) and may be almost impossible to estimate (Boundy-Mills 2006). Experimental procedures used during this investigation also involved selecting yeast colonies for isolation based on their morphology, and discarding colonies with duplicate morphologies for each alga sampled in a given collection. Although essential for reducing the total number of isolates obtained, this approach is likely to have caused further misrepresentation of the biodiversity of marine yeasts, since different yeast species may exhibit identical morphologies and an individual species may have multiple morphologies (Boundy-Mills 2006). Certain yeasts may have been overlooked because culture-based detection techniques may bias isolate recovery towards rapidly growing strains, influencing the apparent diversity present in the community being analysed (Boundy-Mills 2006). These limitations mean that the yeast isolates obtained were only partially representative of the biodiversity present on each algal sample, and may also have contributed to the relatively similar range of marine yeasts isolated between collections. Additional culture-independent and PCR-based techniques allow direct sequencing of nonculturable fungi from environmental samples and may improve understanding of yeast biodiversity (Deák 2006; Singh et al. 2010); evaluation of even limited diversity might be possible with the use of group-specific environmental PCR plus cloning and gene library construction (Bass et al. 2007). It has also been recommended that a polyphasic approach using both culture-based and culture-independent methods be used to maximise the advantages of both 
techniques (Boundy-Mills 2006). Thus, additional biodiversity might be revealed upon further investigation of marine yeasts from the algal samples collected, particularly if nonculturable varieties are analysed (sterile seawater washings of original algal samples have been frozen and retained for this purpose).

The sole use of ITS sequence data is unlikely to have captured the full biodiversity of marine yeasts isolated during this investigation. The ITS region is often used for species delimitation, even though assessment of marine yeast biodiversity using genetic sequence data from just one region such as ITS is limited (Gazis et al. 2011, plus references therein). During an investigation into the delimitation of species boundaries in tropical terrestrial endophytes, it was noted that the choice of molecular marker, the way the molecular marker is used and subjective placement of species boundaries are influential on estimates of biodiversity (Gazis et al. 2011). Sole use of the ITS region for species delimitation has been recognised as restricted, since it generally underestimates the predicted number of species present compared with predictions made using other nuclear loci (Gazis et al. 2011). Yeasts may also possess diversity that is not apparent in genetic analysis; one investigation found that homology between the ITS and LSU sequences of an isolate and those in GenBank do not imply identity, and that fungi isolated from deep sea sediments may have physiological differences which were not detected via analysis of these sequences (Singh et al. 2010). Thus, use of the ITS region for analysis of isolates obtained during this investigation is likely to have resulted in an underestimate of their biodiversity, and assumption of homology between potential species may have contributed to the apparent absence of biogeographic patterns. It has been suggested that analysing isolates with multiple primers is essential when evaluating their biodiversity and ecology, and might reveal patterns of 
biogeography (Nagano et al. 2010; Gazis et al. 2011). Further investigation of isolates with more than one gene or a higher ITS similarity threshold might resolve yeasts into units which more accurately reflect their genetic affinities, and provide a more accurate estimate of biodiversity (Gazis et al. 2011). Since the current taxonomic resolution used can overlook endemic ecophysiological traits and genomic variance, analysis of isolates at a finer taxonomic level might reveal evidence of geographic barriers on microorganism distribution which have so far been overlooked (Boenigk et al. 2006; Gazis et al. 2011).

\subsection{Implications}

Fungi are recognised as principal degraders of biomass in terrestrial ecosystems (Bass et al. 2007) and marine yeasts are suggested to have a similar saprophytic role (Fell 2001). Fungi play a vital role in decomposition and have also been recognised as symbionts or parasites associated with plants and algae (Dix and Webster 1995; Hawksworth 1998). Marine fungi have previously been detected from a range of ecosystems and interact with many components of their environment, including their hosts, competitors and substrates (Dix and Webster 1995). In addition to playing a role in the detrital food web, marine yeasts may contribute to various ecological activities: decomposition of plant substrates, biodegradation of pollutants such as oils, nutrient-recycling, and parasitism of marine animals (Kutty and Philip 2008, plus references therein). Due to the limited information available on the role of individual yeast species, the ecological implications of specific marine yeasts isolated during this investigation are unclear. However, the species of 
yeast present may not be important (Fell 1976); marine yeasts might play a collective, saprophytic role in New Zealand waters.

Certain marine yeast species have been recognised as pathogens or killer yeasts, both of which may have implications in aquaculture settings. Many isolates obtained during this investigation were from the Metschnikowia; Metschnikowia is one of many pathogenic yeast genera (Fell 1976) and includes yeasts known to actively attack their host via forcible spore discharge (Lachance et al. 1976). For example, various species from the genus Metschnikowia parasitize marine copepods (Seki and Fulton 1969) and have been identified as the cause of fry mortality in Chinook salmon Oncorhynchus tshawytscha (Walbaum) (Moore and Strom 2003). Pathogenic marine yeasts have the potential to cause damage and are of particular concern in immunocompromised hosts or stressful situations such as aquaculture settings (Wang et al. 2007). For example, Metschnikowia bicuspidata causes milky disease in the crab Portunus trituberculatus and is responsible for great economic losses (Wang et al. 2007). In contrast, marine yeasts from a wide variety of genera have the ability to produce killer toxin, which kills yeasts of the same or related species without harming the killer strain itself (Magliani et al. 1997). For example, marine yeasts from Williopsis saturnus (Klöcker) Zender and the genus Pichia produce killer toxin against Metschnikowia bicuspidata (Wang et al. 2007; Peng et al. 2010). The killer toxin produced by Williopsis saturnus degrades the cell wall of sensitive yeasts and may be suitable for biocontrol purposes against pathogenic yeasts in organisms such as animals, which are not affected by the toxin due to their lack of a cell wall (Peng et al. 2010). Further information on pathogenic and killer marine yeasts from New Zealand may be valuable to the aquaculture industry; killer marine yeasts might provide potential strategies for countering pathogenic yeasts 
and reducing economic losses (Peng et al. 2010). If marine yeasts with the killer phenotype are isolated in New Zealand, they are likely to be suited to local conditions and would not pose any biosecurity risks if applied in the aquaculture industry.

Potential biocontrol ability against postharvest fungal disease has been demonstrated by marine yeasts (Wang et al. 2008), such as those from genera frequently isolated during this investigation. Yeasts are generally preferred over bacteria for biocontrol purposes, as they are unlikely to cause disease when consumed by humans (Demain et al. 1998). The application of marine yeasts appears to effectively control postharvest disease and avoids the development of fungicide resistance by pathogens (Chi et al. 2009). Marine Rhodosporidium paludigenum, Cryptococcus laurentii (Kuff.) C.E. Skinner and Rhodotorula glutinis demonstrated biocontrol ability against postharvest grey mold caused by Botrytis cinerea on cherry tomatoes, and Rhodosporidium paludigenum was the most effective at decreasing disease incidence (Wang et al. 2010). Certain terrestrial strains of Aureobasidium pullulans also demonstrated activity against postharvest decay on a range of fruits (Schena et al. 1999). Cryptococcus, Rhodotorula, and Aureobasidium isolates obtained during this investigation may have similar biocontrol abilities, and could be used in New Zealand to protect fresh produce from postharvest decay.

A range of marine yeast genera, similar to those detected during this investigation, have previously demonstrated biodegradation abilities that could enable bioremediation of pollutants such as hydrocarbons (Chi et al. 2010). Yeasts and other fungi have been readily detected in oil contaminated environments, giving rise to the suggestion that they may play an important role in the degradation of oil (Fedorak et al. 1984; Zinjarde and Pant 2002; Raghukumar 2008). 
Although no single microorganism is known to be capable of completely degrading crude oil, certain yeast species may contribute to the degradation of oil in marine environments (Ahearn and Meyers 1976). For example, Cryptococcus neoformans (San Felice) Vuill. and species from the genus Rhodotorula were found to degrade hydrocarbons (Da Costa and D'Souza 1979), and a range of genera including Rhodotorula have potential for the biotransformation of polycyclic aromatic hydrocarbons (MacGillivray and Shiaris 1993). It has been suggested that fungi from polluted marine environments are well suited for bioremediation of these conditions (Raghukumar 2002); although unable to compete with hydrocarbon-degrading bacteria under normal conditions, yeasts may have the advantage in challenging environments where the nutrient and oxygen content is low (Fedorak et al. 1984). Fungi are known to be tolerant of challenging conditions such as the high salt concentrations present in marine environments (Raghukumar 2002). Certain marine yeasts also possess heavy metal tolerance and have potential for bioremediation of waters contaminated with toxic metals (Abe et al. 2001). For example, high tolerance against $\mathrm{Cu}^{2+}$ was demonstrated by a deep sea yeast from the genus Cryptococcus (Abe et al. 2001). Thus, the biodegradation abilities of marine yeasts and their tolerance to challenging environmental conditions indicate potential for use in the remediation of various polluted marine environments.

The biodegradation of organic matter into yeast biomass has also been demonstrated by marine yeasts (Rhishipal and Philip 1998), such as those from genera frequently isolated during this investigation. Single cell yeast biomass is of high nutritional quality and a cost effective choice for supplementing animal or aquaculture feed (Rhishipal and Philip 1998). For example, Cryptococcus aureus is considered to be an excellent candidate for single cell protein production 
due to its ease of cultivation, good growth on inulin and beneficial nutritional properties (Chi et al. 2008). Yeast biomass production is efficient due to their short generation time, limited nutrient requirements (Kutty and Philip 2008, plus references therein), and the ease of managing production and concentration of yeast cells on a large scale (Chi et al. 2007). Certain yeasts also thrive on cheap waste products; yeasts from the genus Rhodotorula were excellent candidates for the production of single cell protein from prawn shell waste (Rhishipal and Philip 1998). Some marine yeasts produce biomass more efficiently in seawater rather than distilled water (Singh et al. 2010), so marine yeast biomass production has the advantage of using seawater, which is an abundant resource (Chi et al. 2006). Due to their various advantages, marine yeasts may have potential for development as a low cost feed supplement for use in New Zealand's aquaculture industry.

Many marine yeasts produce compounds with potential applications as pharmaceuticals, cosmetics and industrial chemicals (Chi et al. 2006). Applications have previously been described for all marine yeast genera frequently isolated during this investigation: Cryptococcus, Rhodotorula, Metschnikowia and Aureobasidium (Chi et al. 2009, plus references therein). For example, a marine strain of Cryptococcus aureus demonstrated secretion of large amounts of inulinase, which can be used for the production of ultra high fructose syrups and high concentrations of ethanol (Gao et al. 2007, plus references therein). Aureobasidium pullulans is also biotechnologically important due to its ability to produce various extracellular enzymes: proteinase, amylase, lipase, cellulase, xylanase, mannanase and transferases (Chi et al. 2009, plus references therein). Enzymes produced by marine yeasts may have unique properties; subtle physical and chemical differences were observed between inulinases produced by marine and 
terrestrial strains of the same species (Chi et al. 2009, plus references therein). Production of marine yeast byproducts has the advantage of using seawater (Chi et al. 2006), and their short generation time and limited nutrient requirements make marine yeasts an excellent economic choice (Kutty and Philip 2008, plus references therein). Screening fungi from extreme marine environments may yield additional novel enzymes and bioactive molecules which could be exploited for use in biotechnological processes (Raghukumar 2008).

\subsection{Conclusions}

Although this was the first known investigation of marine yeast biodiversity from waters surrounding New Zealand's main Islands, the yeasts isolated were not unique. Marine yeasts were isolated and identified from a diverse range of species that have been described during previous investigations. High ITS sequence similarity was observed between yeasts isolated during this investigation and those from diverse marine and terrestrial substrates worldwide; the high level of similarity may suggest that marine yeasts are ubiquitous at a global level. However, data regarding marine yeasts is insufficient to support or dispute whether they also demonstrate biogeographic distribution patterns. Limitations of the experimental procedures used mean that results obtained during this investigation are only partially representative of New Zealand's marine yeast biodiversity. The marine yeasts isolated may have ecological implications; marine yeasts are generally considered to be saprophytes but some genera are recognised as pathogenic and may have negative implications for aquaculture in New Zealand. Many marine yeast genera isolated during this investigation have previously described applications, including the 
production of nutritionally valuable yeast biomass or useful compounds, biocontrol abilities against postharvest decay, and potential abilities for bioremediation of polluted marine environments. More extensive investigation of new and existing marine yeasts from New Zealand may provide further information regarding their biodiversity, ecological role and potential applications. 


\section{Appendix}

\subsection{Media Components}

Difco Potato Dextrose Agar Plus (PDA):

40 g Difco Potato Dextrose Agar

$10 \mathrm{~g}$ Oxoid Yeast Extract

5 g Oxoid Peptone

Prepared with $1 \mathrm{~L}$ aged seawater

Difco Standard Marine Agar Plus (STD):

37.5 g Difco Marine Agar

5 g Sigma-Aldrich D+ Galactose

$10 \mathrm{~g}$ Oxoid Yeast Extract

5 g Oxoid Beef Extract

5 g Oxoid Peptone

Prepared with $1 \mathrm{~L} \mathrm{ddH}_{2} \mathrm{O}$

Difco Standard Marine Agar Plus 2:

20 g Difco Marine Agar

10 g Sigma-Aldrich D+ Glucose

$1 \mathrm{~g}$ Oxoid Yeast Extract 
4 g Oxoid Beef Extract

$4 \mathrm{~g}$ Pancreatic Digest of Gelatin

$2.5 \mathrm{~g} \mathrm{NaCl}$

Prepared with $1 \mathrm{~L} \mathrm{ddH}_{2} \mathrm{O}$

Difco Standard Marine Agar Plus 3:

13.3 g Difco Marine Agar

15 g Sigma-Aldrich D+ Glucose

$0.5 \mathrm{~g}$ Oxoid Yeast Extract

0.5 g Oxoid Peptone

0.5 g Gelatin Hydrolysate

Prepared with $1 \mathrm{~L} \mathrm{ddH}_{2} \mathrm{O}$

Difco Marine Broth Plus:

30 g Difco Marine Broth

5 g Sigma-Aldrich D+ Galactose

10 g Oxoid Yeast Extract

5 g Oxoid Beef Extract

5 g Oxoid Peptone

Prepared with $1 \mathrm{~L} \mathrm{ddH}_{2} \mathrm{O}$

Sigma Luria Broth:

Prepared according to manufacturer's instructions with aged seawater 


\subsection{Optimisation of Culture Techniques}

Prior to Collections 1-4, a preliminary investigation was conducted in order to determine which culture techniques yielded the highest number of marine yeast colonies. Various media, antibiotics and inoculation methods were trialled using a piece of miscellaneous alga. More colonies were observed on agar plates inoculated using the homogenisation of alga method (a $\sim 2$ $\mathrm{x} 2 \mathrm{~cm}$ algal sample was homogenised with a mini-blender in a falcon tube with $\sim 10 \mathrm{ml}$ of sterile seawater, and a $100 \mu \mathrm{l}$ aliquot of the resultant solution was transferred to an agar plate) than agar plates inoculated with a $100 \mu \mathrm{l}$ aliquot of seawater (Table 9). The number of colonies obtained on each agar plate was not excessive, rendering sample dilution unnecessary. More colonies were observed on Difco Standard Marine Agar Plus (STD) and Difco Potato Dextrose Agar Plus (PDA) than the other two media trialled (Table 10). Antibiotic Treatment B (100 $\mu 1$ of $10 \mathrm{mg} \mathrm{ml}^{-1}$ streptomycin sulfate solution (in distilled water) plus $100 \mu \mathrm{lof} 5 \mathrm{mg} \mathrm{ml}^{-1}$ rifampicin solution (in methanol)) was found to be the most effective for suppression of bacterial overgrowth (Table 10). Due their efficacy in preliminary investigations, STD and PDA plates treated with both antibiotics (streptomycin sulfate and rifampicin) were used for yeast isolation in Collections 1-4. During Collection 1, $100 \mu \mathrm{l}$ of streptomycin sulfate solution with concentration $10 \mathrm{mg} \mathrm{ml}^{-1}$ and $100 \mu \mathrm{l}$ of rifampicin solution with concentration $5 \mathrm{mg} \mathrm{ml}^{-1}$ were applied to each plate. During Collections 2, 3 and 4, $100 \mu \mathrm{l}$ of streptomycin sulfate solution with concentration of $9 \mathrm{mg} \mathrm{ml}^{-1}$ and $100 \mu \mathrm{l}$ of rifampicin solution with concentration of $4.5 \mathrm{mg} \mathrm{ml}^{-1}$ were applied to each plate. 
Table 9. Appearance of agar plates after inoculation with seawater or the solution resulting from homogenisation of an algal sample in sterile seawater.

\begin{tabular}{|l|l|l|l|l|}
\hline \multicolumn{1}{|c|}{$\begin{array}{c}\text { Aliquot Applied } \\
\text { to Agar Plate }\end{array}$} & $\begin{array}{c}\text { Number of } \\
\text { Colonies on } \\
\text { Agar Plate 1 } \\
\text { (STD) }\end{array}$ & $\begin{array}{c}\text { Number of } \\
\text { Colonies on } \\
\text { Agar Plate 2 } \\
\text { (STD) }\end{array}$ & $\begin{array}{c}\text { Number of } \\
\text { Colonies on } \\
\text { Agar Plate 3 } \\
\text { (STD) }\end{array}$ & $\begin{array}{c}\text { Average } \\
\text { Number of } \\
\text { Colonies per } \\
\text { Agar Plate }\end{array}$ \\
\hline $100 \mu$ l Seawater & 17 & 13 & 16 & 15 \\
\hline $\begin{array}{l}100 \mu \text { Seawater } \\
(1 \text { in 10 Dilution) }\end{array}$ & 3 & 2 & 1 & 2 \\
\hline $\begin{array}{l}100 \mu \text { l Homogenised } \\
\text { Alga Solution }\end{array}$ & $\sim 7069$ & $\sim 7304$ & $\sim 7854$ & $\sim 7383$ \\
\hline $\begin{array}{l}100 \mu \text { l Homogenised } \\
\text { Alga Solution } \\
(1 \text { in 10 Dilution) }\end{array}$ & $\sim 2592$ & & $\sim 3142$ & $\sim 2670$ \\
\hline
\end{tabular}

Table 10. Appearance of agar plates after treatment with antibiotics and inoculation with the solution resulting from homogenisation of an algal sample in sterile seawater.

\begin{tabular}{|c|c|c|c|c|}
\hline Antibiotic Treatment & $\begin{array}{c}\text { Difco Potato } \\
\text { Dextrose } \\
\text { Agar Plus } \\
\text { (PDA) }\end{array}$ & $\begin{array}{c}\text { Difco } \\
\text { Standard } \\
\text { Marine Agar } \\
\text { Plus (STD) }\end{array}$ & $\begin{array}{c}\text { Difco } \\
\text { Standard } \\
\text { Marine Agar } \\
\text { Plus } 2\end{array}$ & $\begin{array}{c}\text { Difco } \\
\text { Standard } \\
\text { Marine Agar } \\
\text { Plus } 3\end{array}$ \\
\hline $\begin{array}{l}\text { Treatment A: } \\
50 \mu \mathrm{l} \mathrm{Vancomycin} \mathrm{Solution} \\
\left(25 \mathrm{mg} \mathrm{ml}^{-1} \text { in Distilled Water }\right)\end{array}$ & $\begin{array}{l}\text { Heavy } \\
\text { Bacterial } \\
\text { Lawn }\end{array}$ & $\begin{array}{l}\text { Heavy } \\
\text { Bacterial } \\
\text { Lawn }\end{array}$ & $\begin{array}{l}\text { Light } \\
\text { Bacterial } \\
\text { Lawn }\end{array}$ & $\begin{array}{l}\text { Light } \\
\text { Bacterial } \\
\text { Lawn }\end{array}$ \\
\hline $\begin{array}{l}\text { Treatment B: } \\
100 \mu 1 \text { Streptomycin Sulfate } \\
\text { Solution } \\
\left(10 \mathrm{mg} \mathrm{ml}^{-1} \text { in Distilled Water) }\right. \\
+100 \mu 1 \mathrm{Rifampicin} \mathrm{Solution}^{-1} \text { in Methanol) }\end{array}$ & $\begin{array}{l}\text { Many Fungal } \\
\text { Colonies }\end{array}$ & $\begin{array}{l}\text { Many Fungal } \\
\text { Colonies }\end{array}$ & $\begin{array}{l}\text { Some Fungal } \\
\text { Colonies }\end{array}$ & $\begin{array}{l}\text { Some Fungal } \\
\text { Colonies }\end{array}$ \\
\hline $\begin{array}{l}\text { Treatment C: } \\
\text { Control } \\
\text { (No Antibiotic Treatment) }\end{array}$ & $\begin{array}{l}\text { Very Heavy } \\
\text { Bacterial } \\
\text { Lawn }\end{array}$ & $\begin{array}{l}\text { Very Heavy } \\
\text { Bacterial } \\
\text { Lawn }\end{array}$ & $\begin{array}{l}\text { Heavy } \\
\text { Bacterial } \\
\text { Lawn }\end{array}$ & $\begin{array}{l}\text { Light } \\
\text { Bacterial } \\
\text { Lawn }\end{array}$ \\
\hline
\end{tabular}




\subsection{Geneious Alignments by Subphylum}

\subsubsection{Pezizomycotina}

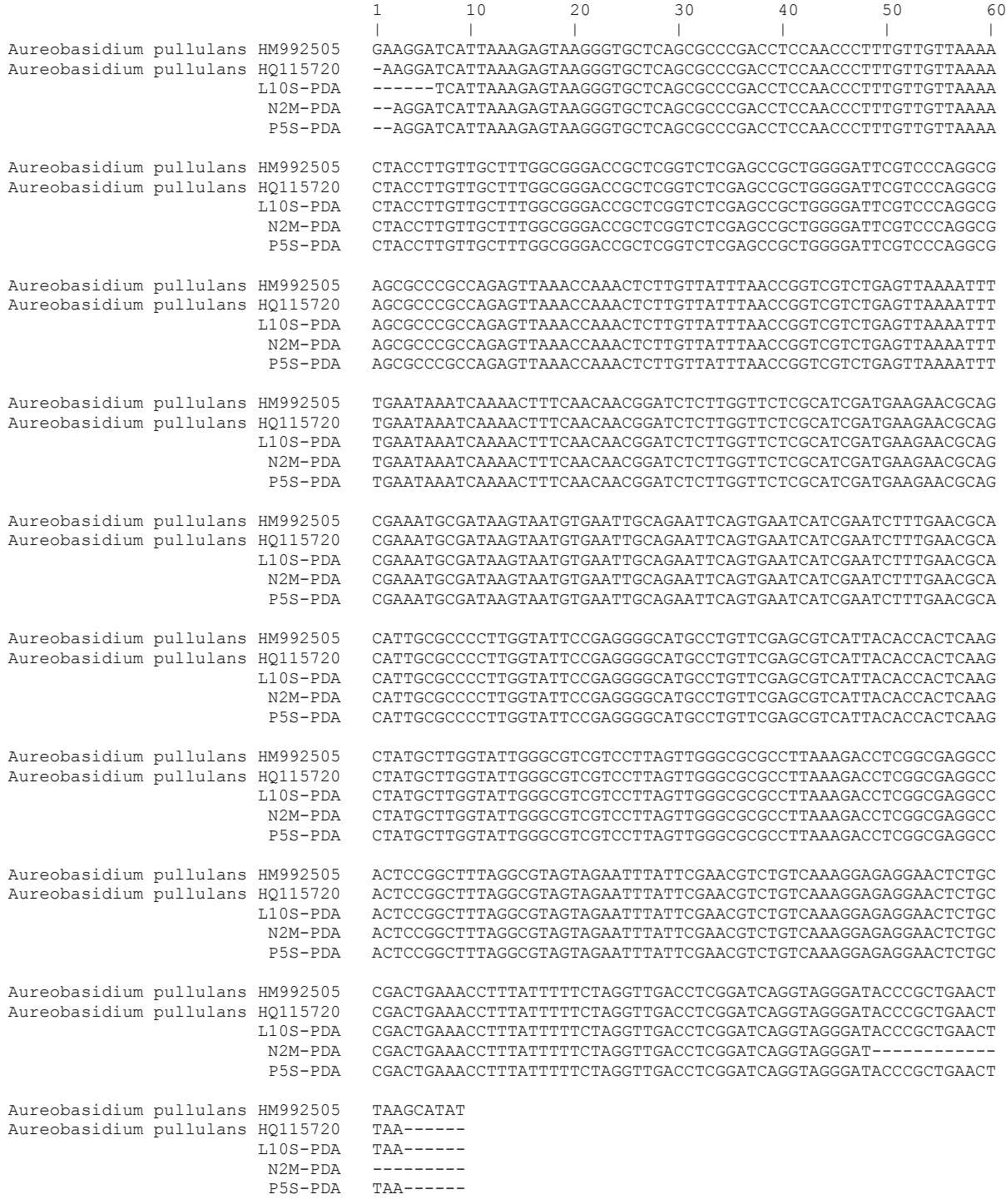




\subsubsection{Saccharomycotina}

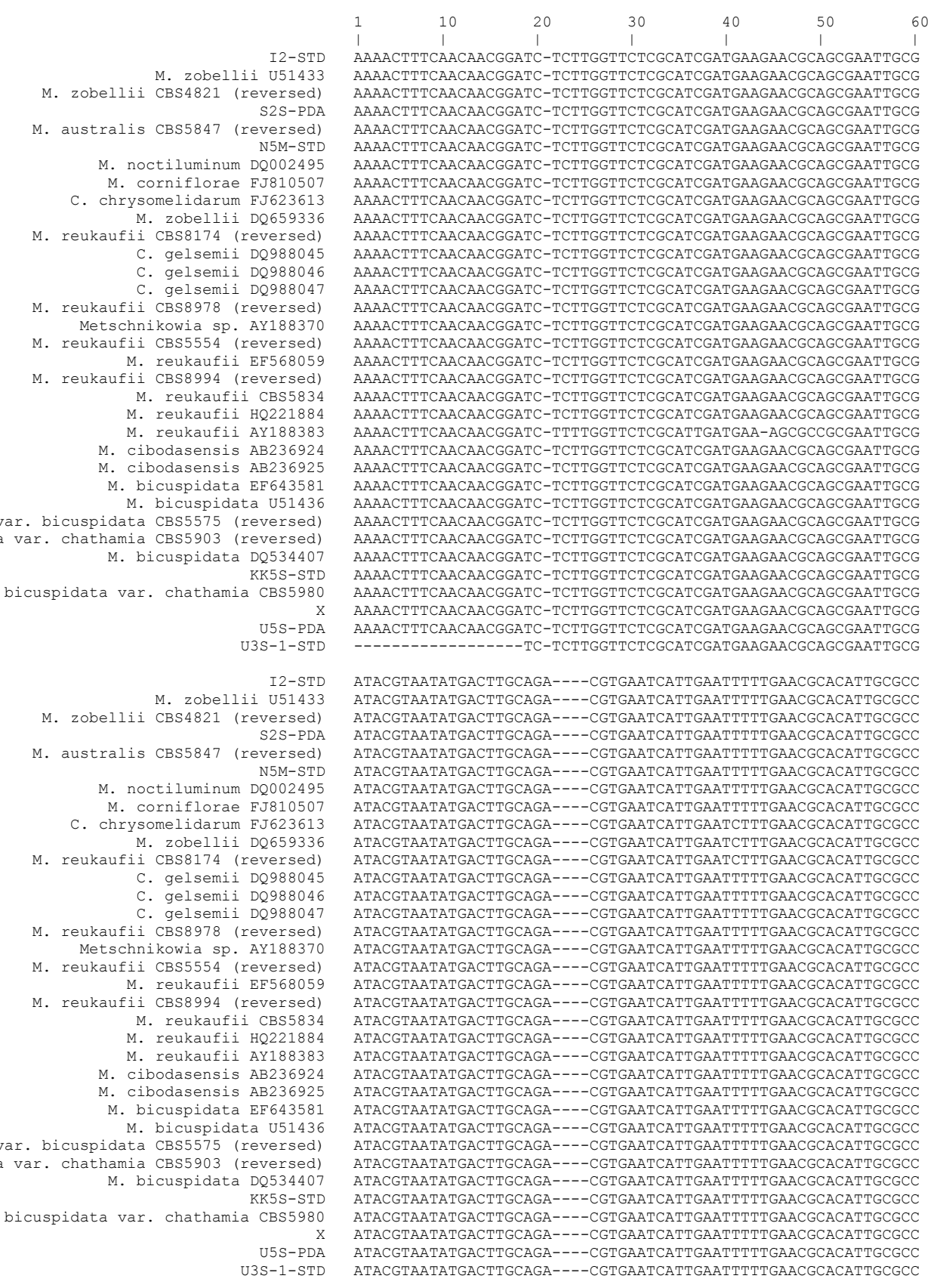


I2-STD M. zobellii U51433 M. zobellii CBS4821 (reversed) S2S-PDA

M. australis CBS5847 (reversed) N5M-STD

M. noctiluminum DQ002495 M. corniflorae FJ810507 C. chrysomelidarum FJ623613 M. zobellii DQ659336 M. reukaufii CBS8174 (reversed) C. gelsemii DQ988045 C. gelsemii DQ988046 C. gelsemii DQ988047 M. reukaufii CBS8978 (reversed) Metschnikowia sp. AY188370

M. reukaufii CBS5554 (reversed) M. reukaufii EF568059

M. reukaufii CBS8994 (reversed) M. reukaufii CBS5834 M. reukaufii HQ221884 M. reukaufii AY188383 M. cibodasensis AB236924

M. Cibodasensis AB236925

M. bicuspidata EF643581 M. bicuspidata U51436 M. bicuspidata var. bicuspidata CBS5575 (reversed) M. bicuspidata var. chathamia CBS5903 (reversed)

M. bicuspidata DQ534407

M. bicuspidata var. chathamia CBS5980 U5S-PDA U3S-1-STD I2-STD M. zobellii U51433 M. zobellii CBS4821 (reversed) S2S-PDA

M. australis CBS5847 (reversed) M. noctiluminum DQ002495 C. chrysomelidarum FJ623613 M. zobellii DQ659336 M. reukaufii CBS8174 (reversed) C. gelsemii DQ988045 C. gelsemii DQ988046 C. gelsemii DQ988047 M. reukaufii CBS8978 (reversed) Metschnikowia sp. AY188370

M. reukaufii CBS5554 (reversed) M. reukaufii EF568059

M. reukaufii CBS8994 (reversed) M. reukaufii CBS5834 M. reukaufii HQ221884 M. reukaufii AY188383 M. Cibodasensis AB236924 M. cibodasensis AB236925 M. bicuspidata EF643581 M. bicuspidata U51436 M. bicuspidata var. bicuspidata CBS5575 (reversed) M. bicuspidata var. chathamia CBS5903 (reversed)

M. bicuspidata DQ534407 KK5S-STD

M. bicuspidata var. chathamia CBS5980 U5S-PDA U3S-1-STD
TTAAGGTATTCCTCAAGGCATGCGTGGATGAGCG-ATATT-TACT-CTCAAACCACT-TG TTAAGGTATTCCTCAAGGCATGCGTGGATGAGCG-ATATT-TACT-CTCAAACCACT-TG TTAAGGTATTCCTCAAGGCATGCGTGGATGAGCG-ATATT-TACT-CTCAAACCACT-TG TTAAGGTATTCCTCAAGGCATGCGTGGATGAGCG-ATATT-TACT-CTCAAACCACT-TG TTGGGGTATTCCCCAAGGCATGCGTGGATGAGCG-ATATT-TCCT-CTCAAACCTAT--G TTAAGGTATTCCTCAAGGCATGCGTGGATGAGCG-ATATT-TACT-CTCAAACCACT-TG TTAAGGTATTCCTCAAGGCATGCGTGAATGAGCG-ATATT-TACT-CTCAAACCCCTAGC TTAAGGTATTCCTCAAGGCATGCGTGAATGAGCG-ATATT-TACT-CTCAAACCCCTAGG TTAAOCTATTCCTCAAGCCATGCGTGAATGAGCG -ATATT-TACC-CTCAAACCTTT-U G TTAAGGTATTCCTCAAGGCATCCGTGAATGAGCG-ATATT-TACC-CTCAAACCTTT--G TTAAGGTATTCCICAAGGCATGCGIGAATGAGCG-ATATT-TACC-CTCAAACCTTT-作 ITAAGGTATTCCTCAAGGCAIGCGCGGATGAGCG-ATATT-TACT-CTCAAACCTCT-TTAAGGTATTCCTCAAGGCATGCGCGGATGAGCG-ATATT-TACT-CTCAAACCTCT-TTAAGGTATTCCTCAAGGCATGCGCGGATGAGCG-ATATT-TACT-CTCAAACCTCT--G TTAAGGTATTCCTCAAGGCATGCGTGAATGAGCG-ATGTT-TATT-CTCAAACCTCA-TTAAGGTATTCCTCAAGGCATGCGTGGATGAGCG-ATATT-TACT-CTCAAACC--TTCC TTAAGGTATTCCTCAAGGCATGCGTGGATGAGCG-ATATT-TACT-CTCAAACCTAT--G TTAAGGTATTCCTCAAGGCATGCGTGGATGAGCG-ATATT-TACT-CTCAAACCTAT--G TTAAGGTATTCCTCAAGGCATGCGTGGATGAGCG-ATATT-TACT-CTCAAACCTAT--G TTAAGGTATTCCTCAAGGCATGCGTGGATGAGCG-ATATT-TACT-CTCAAACCTAT--G TTAAGGTATTCCTCAAGGCATGCGTGGATGAGCG-ATATT-TACT-CTCAAACCTAT-TTAAGGTATTCCTCAAGGCATGCGTGGGTGAGCG-ATATT-TACT-CTCAAACCTAT-GE TTAAGGTATTCCTCAAGGCATGCGTGGGTGAGCG-ATATT-TACT-CTCAAACCTAT-GG TTAAGGTATTCCTCAAGGCATGCGTGGATGAGCG-ATATT-TACT-CTCAAACCTCT-TG TTAAGGTATTCCTCAAGGCATGCGTGGATGAGCG-ATATT-TACT-CTCAAACCTCT-TG TTAAGGTATTCCTCAAGGCATGCGTGGATGAGCG-ATATT-TACT-CTCAAACCTCT-TG
TTAAGGTATTCCTCAAGGCATGCGTGGATGAGCG-ATATT-TACT-CTCAAACCTCT-TG TTAAGGTATTCCTCAAGGCATGCGTGGATGAGCG-ATATT-TACT-CTCAAACCTCT-TG TTAAGGTATTCCTCAAGGCATGCGTGGATGAGCG-ATAT-TACI-CICAAACCICT-T TTAAGGTATTCCTCAAGGCATGCGTGGATGAGCGGATATT-TACT-CTCAAACCTCT-TG TTAAGGTATTCCTCAAGGCATGCGTGGATGAGCG-ATATT-TACT-CTCAAACCTCT-TG TTAAGGTATTCCTCAAGGCATGCGTGGATGAGCG-ATATT-TACT-CTCAAACCTCT-TG TTAAGT 作 TTAAGGTATTCCTCAAGGCATGCGTGGATGAGCG-ATATT-TACT-CTCAAACCTAT-TG
TTAAGGTATTCCTCAAGGCATGCGTGGATGAGCG-ATATT-TACT-CTCAAACCTAT-TG

GTTTGGTCTTGGCCCCCTTTTTTTTCATAGGGCCTAAATATCAAAT-GGTCTCTAG-AATA GTTTGGTCTTGGCCCCCTTTTTTTTCATAGGGCCTAAATATCAAATGGTCTCT-AG-AATA GTTTGGTCTTGGCCCCCTTTTTTTTCATAGGGCCTAAATATCAAATGGTCTCT-AG-AATA GTTTGGTCTTGGCCCCCTT-TTTTTCATAGGGCCTAAATATCAAATGGTCTCT-AG-AATA GTTTGGTCTTGAC--CCTT---------GGTCCAAAATATCAACTAGAATCT-AG-AATA GTTTGGTCTTGGCCCCCTTTT-TTTCATAGGGCCTAAATATCAAATGGTCTCT-AG-AATA GTTTGGTCCTGGCC-C--------CCTACAGCCTAAACATCAACT-GATTTT--AAAATA GTTTGGTCCTGGCC-C--_-_---_CCTACAGCCTAAACATCAACT-GATTTT--AAAATA GTTTGGTTTTGGCCCT--.---.--GGCCTCAATATCAACT-GGCTCT-AG-AATA GTTTGGTTTTGGCCCT---_---_---GGCTCAATATCAACT-GGCTCI-AG-AATA

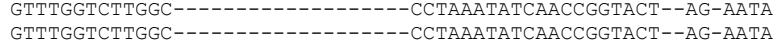

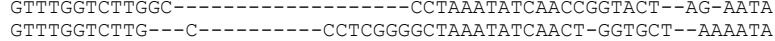
GTTTGGTCTTG---C---------CCTCGGGGCTAAATATCAACT-GGTGCT--AAAATA GTTTGGTCTTG---C---------CCTCGGGGCTAAATATCAACT-GGTGCT--AAAATA GTTTGGCCTTGTC---_-_-_-_---_---TCTAAATATCA-TTAGGCGT--AAAAATA GTTTGGTCTTG----TA-----ACCA-----CAAAATATCAAAT-GGCTGT-AG-AATA GTTTGGTTTTG----T--------AAT----CCTAAATATCAACG-GTATCT-AG-AATA GTTTGGTTTTG----T--------AAT----CCTAAATATCAACG-GTATCT-AG-AATA GTTTGGTTTTG----T--------AAT----CCTAAATATCAACG-GTATCT-AG-AATA GTTTGGTTTTG----T-------AAT---_CTAAATATCAACG-GTATCT-AG-AATA GTTTGGTTITG--1 GT-TGGGTTTG----T-_-_-_-AAT---CCTAAATATCAACG-GTATCT-AG-AATA GTTTGGTTTTG----T--------AAT----CCTAAATATCAACG-GTATCT-AG-AATA GTTTGGTTTTG----T--------AAT----CCTAAATATCAACG-GTATCT-AG-AATA GTTTGGTCTTGAC--TC------CCAA--GGTCTAAATATCAAAT-GTCTCT-AG-AATA GTTTGGTCTTGAC--T-------CCA---GGTCTAAATATCAAAT-GACTCT-AG-AATA GTTTGGTCTTGAC--T-------CCA---GGTCTAAATATCAAAT-GACTCT-AG-AATA GTTTGGTCTTGAC--T-------CCA---GGTCTAAATATCAAAT-GTCTCT-AG-AATA GTTTGGTCTTGAC--TC------CCAA--GGTCTAAATATCAAAT-GTCTCT-AG-AATA GTTTGGTCTTGAC-T---------CCA---GGTCTAAATATCAAAT-GTCTTC------GTTTGGTCTTGAC----T------CCA---GGTCTAAATATCAAAT-GTCTCT-AG-AATA GTTTGGTCTTGAC--T-------_CCA---GGTCTAAATATCAAAT-GTCTCT-AG-AATA GTTTGGTCTTGAC---T-------CCA---GGTCTAAATATCAAAT-GTCTCT-AG-AATA GTTTGGTCTTGAC---T------CCA---GGTCTAAATATCAAAT-GTCTCT-AG-AATA 


\subsubsection{Agaricomycotina}

R. minuta AF190010 R. slooffiae AF444552 R. slooffiae AF444627 Cy. macerans AF444317 Cy. macerans AF444329 Uncultured fungus AB520409 Uncultured fungus AB520402 Uncultured fungus AB520396

C. sp. AF 444448 P4M-STD

$$
\begin{array}{r}
\text { P4M-STD } \\
\text { T1S-1-PDA }
\end{array}
$$

C. macerans EU082232

C. macerans EU082233

C. macerans EU082231

C. macerans AY052490 Uncultured fungus EF504408 Bm. albus AF444664

B. unica AF444441 Bm. albus AB093527

$\mathrm{Bm}$. albus CBS7856

C. dimennae AY188365

C. dimennae AY188365

C. dimennae AF410473 Uncultured Fungus AM901805

$$
\begin{array}{r}
\text { T1S-3-PDA } \\
\text { Y5S-PDA }
\end{array}
$$$$
\text { Y } 5 \text { S-PDA }
$$

C. oeirensis AY188362

C. magnus AF190009 Y $2 \mathrm{M}-\mathrm{PDA}$

C. oeirensis AF444349

C. oeirensis AF444364

C. oeirensis EU002866

C. Oeirensis EU002866

C. chernovii AF444354 Uncultured fungus FM178256 U3S-PDA

Uncultured fungus AJ87538 Uncultured fungus AB520331

C. carnescens AB035050 Uncultured fungus FJ820684 Uncultured fungus AM901777

Z2S-STD
C. carnescens EU149785 C. sp. HM849618

C. carnescens CBS8395

C. carnescens AB105438

C. victoriae AY188380

C. victoriae AJ581046

C. victoriae AM160647

C. victoriae CBS9265

C. victoriae HM849622

C. victoriae AF444645

C. victoriae AY04065 L2S-1-PDA $5 S-4-S T D$

C. sp. HM589279

C. victoriae AY040654 S4S-PDA

C. victoriae CBS9267 $\begin{array}{ccccccc}1 & 10 & 20 & 30 & 40 & 50 & 60 \\ 1 & 1 & 1 & 1 & 1 & 1 & 1\end{array}$

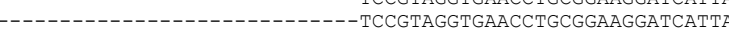

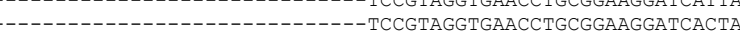
(1) TCCGTAGGTGACCTGCGGAAGGATCACTA (1) 作

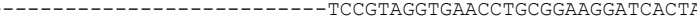

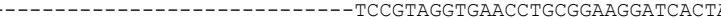

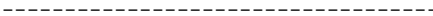
ThAGGACACIA -AAGGATCACT

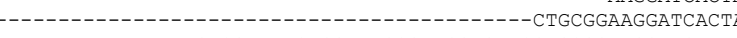
---------AGTCGTA--CAGGTTTCCGTAGGTGAACCTGCGGAAGGATCATTA -

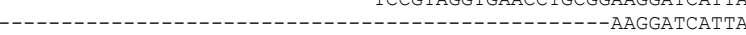
TCCGTAGGTGAACCTGCGGAAGGATCATTA

TTTAGAGGAAGTAAAAGTCGTAACAAGGTTTCCGTAGGTGAACCTGCGGAAGGATCATTA

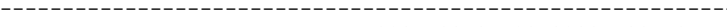
TAGCCCGCCGGACTCAATCATTATCTTTCCT-CGTAGGTGAACCTGCGGAAGGATCATTA TTTAGAGGAAGTAAAAGTCATAACAAGGTTTCCGTAGGTGAACCTGCGGAAGGATCATTA (1) TCCGTAGTGAACCTGCGGAAGGATCATT

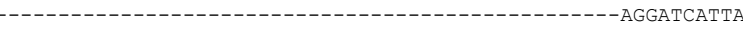

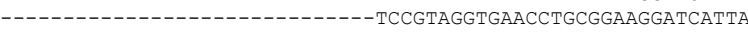

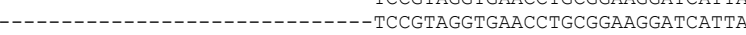

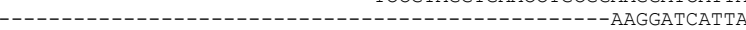
$-1$ TTTAGAGGAAGTAAAAGTCGTAACAAGGTTTCCGTAGGTGAACCTGCGGAAGGATCATTA TTTAGAGGAAGTAAAAGTCGTAACAAGGTTTCCGTAGGTGAACCTGCGGAAGGATCATTA .---AGTAAAAGTCGTAACAAGGTTTCCGTAGGTGAACCTGCGGAAGGATCATTA (1)--AGTAAAAGTCGTAACAAGGTTTCCGTAGGTGAACCTGCGG AAGGATCATTA GGAAGGATCATT

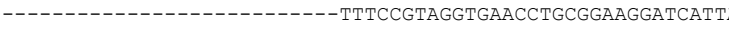
TTTAGAGGAAGTAAAAGTCATAACAAGGTTTCCGTAGGTGAACCTGCGGAAGGATCATT ------------------TTTCCGTAGGTGAACCTGCGGAAGGATCATTA

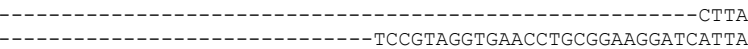

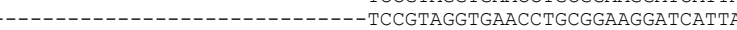
TTTAGAGGAAGTAAAAGTCATAACAAGGTTTCCGTAGGTGAACCTGCGGAAGGATCATTA

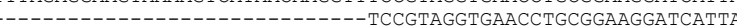
TCCGTAGGTGAACCTGCGGAAGGATCATT -----GAAAGTAAAAGTCGTAACAAGGTTTCCGTAGGTGAACCTGCGGAAGGATCATTA

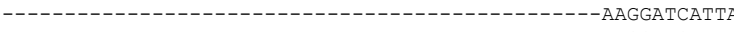
(1)

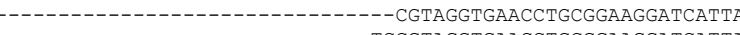
TTTAGAGGAAGTAAAAGTCATAACAAGGTTTCCGTAGGTGAACCTGCGGAAGGATCATTA 
R. minuta AF190010

. Slooffiae AF444552

R. slooffiae AF444627

Cy. macerans AF444317
Cy. macerans AF444329 Uncultured fungus AB520409 Uncultured fungus AB520402 Uncultured fungus AB520396 C. sp. AF444448 P $4 M-S T D$
T1S-1-PDA

C. macerans EU082232

C. macerans EU082233

C. macerans EU082231

C. macerans AY052490 Uncultured fungus EF504408

Bm. albus AF444664 $\mathrm{V} 5 \mathrm{M}-\mathrm{PDA}$

B. unica AF444441

Bm. albus AB093527

Bm. albus CBS7856

C. dimennae AY188365

c. dimennae AF410473

C. dimennae EU266559

Uncultured Fungus AM901805 T1S-3-PDA
Y5S-PDA $\mathrm{Y} 5 \mathrm{~S}-\mathrm{PDA}$
$2 \mathrm{~S}-1-\mathrm{STD}$

C. oeirensis AY188362 C. magnus AF190009 Y $2 \mathrm{M}-\mathrm{PDA}$

C. oeirensis AF 444349

C. oeirensis AF444364

C. oeirensis EU002866

C. chernovii AF444354

C. chernovii FN400936 Uncultured fungus FM178256 U3S-PDA

Uncultured fungus AJ875389 Uncultured fungus AB520331

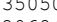
J820684$$
\text { Z2S-STD }
$$

C. sp. HM849618

C. carnescens CBS8395

C. carnescens AB105438

C. victoriae AY188380

c. victoriae AJ581046

C. victoriae AM160647

C. victoriae CBS9265

C. victoriae HM84962

c. victoriae HM849622

C. victoriae AF444645

C. victoriae AY04065 L2S-1-PDA $22 S-1-P D A$
$W 5 S-4-S T D$

C. sp. HM589279

c. victoriae AY04065 S4S-PDA

C. victoriae CBS9267

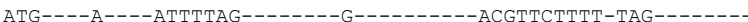
ATG-A-------ATTTTAG----------------GACGTTCTTTTT---------

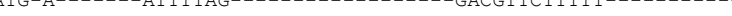
AAG-T-----AAACGCCCTC--C----------GGGGCTCTCTT-TATTC---ACAAG-T------AAACGCCCTC--C-----------GGGGCTCTCTT-TATTC---ACAAG-T-----AAACGCCCTC------------CGGGGCTCTCTT-TATTCAC---AAG-T-----AAACGCCCTC-------------CGGGGCTCTCTT-TATTCAC---AAG-T-----AAACGCCCTC------------CGGGGCTCTCTT-TATTCAC--AAG---T----AAACGCCCTC---C------------GGGGCTCTCTT-TAAT--TCACAAG-T------AAACGCCCTC---C-----------GGGGCTCTCTT-TATTC---AC-

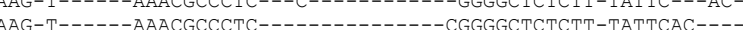
AAG---T----AAACGCCCTC---C---_---_---GGGGCTCTCTT-TATTC---AC AAG---T----AAACGCCCTC---C-----------GGGGCTCTCTT-TATTC---ACAAG---T----AAACGCCCTC---C-----------GGGGCTCTCTT-TAAT--TCACAAG---T----AAACGCCCTC---C---------GGGGCTCTCTT-TAAT--TCAC-

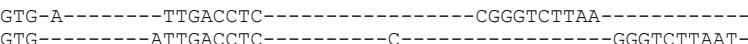

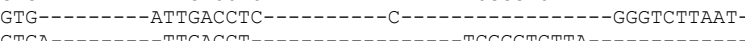

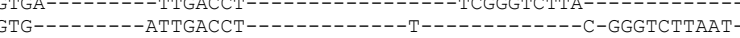

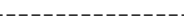

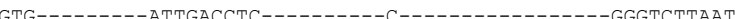

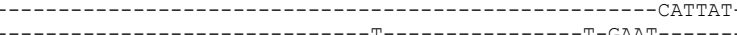

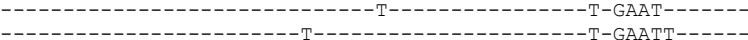
TT--(1) -

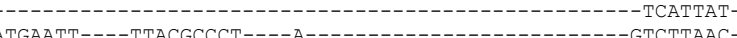
AT----_-----AATT--------T------------AGAT----T-GAACCATAGG-

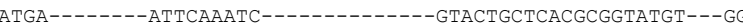
ATG-_-_--_-_TTATA ATG-A-1

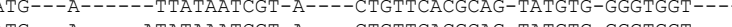
ATG--A-ATATAAATCGI-A---CIGITCACGCAG-TAIGIG-GGGTGGT--TTG-----AATTCAAATCATC-T-------GTGGCTTCGGTCCGGAT-GTGCCAACAG

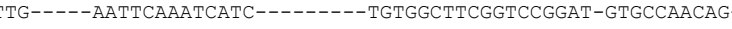
TTGAATT---CAAATCATCTG----------TGGCTTCGGTCCGGATGTGCCAACAGG TTGAATT---CAAATCATCTG-----------TGGCTTCGGTCCGGATGTGCCAACAGTTGAATT---CAAATCATCTG-----------TGGCTTCGGTCCGGATGTGCCAACAGTTGAATT---CAAATCATCTG---------TGGCTTCGGTCCGGATGTGCCAACAG---_----ATAATGCTCTC-----------TGGCTTCGGTCAGTT-GAGTT----CАT--A--------ATGCTCTC------------TGGCTTCGGTCAGTTGAGTTCAATGAG

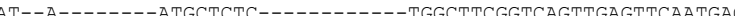
AT-A AT-A----- ATGCTCTC-----1 TGGCTTCGGTCAGTTGAGTTCAATGA AT-TGGCTTCGGTCAGCT-GAGT--TCAA AT----A-----ATGCTCTC---T-------GGCTTCGGTCAGTT-GAGT--TCAAAT--------AATGCTCTC----------TGGCTTCGGTCAGTT-GAGT--TCAAAT---------AATGCTCTC----------TGGCTTCGGTCAGTT-GAGT--TCAAAT----A------GTGCCCT----T--------GATGCAAATCA-TT-GGGTT----AT----A------GTGCCCAT---T--------GATGCAAATCA-TT-GGGTT-----AT----A-----GTGCCCAT---T--------GATGCAAATCA-TT-GGGTT----AT--A-------GTGCCCTC--T---------GATGCAAATCA-TT-GGGTT----AT----A------GTGCCCTC---T---------GATGCAAATCA-TT-GGGTT----AT----A------GTGCCCTC---T--_-----_GATGCAAATCA-TT-GCGTT----AT--- A-- -0 GTGCCCTC-AT-- AAT

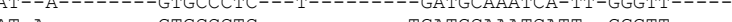
AT-A------_GIGCCCTC----------TGATGCAAATCATI--GGGIT----AT----A-----GTGCCCTC---T-------GATGCAAATCA-TT-GGGTT----AT----A-----GTGCCCTC---T-------_GATGCAAATCA-TT-GGGTT----AT-A--------GTGCCCTC---------- TGATGCAAATCATT--GGGTT----- 
R. minuta AF190010

. Slooffiae AF444552

R. slooffiae AF444627

Cy. macerans AF444317 Cy. macerans AF444329 Uncultured fungus AB520402 Uncultured fungus AB520396 C. sp. $A F 444448$

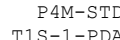

C. macerans EU082232

C. macerans EU082233

C. macerans EU082231

C. macerans AY052490 Uncultured fungus EF50440

Bm. albus AF444664 V5M-PDA

B. unica AF444441

Bm. albus AB093527

$\mathrm{Bm}$. albus CBS7856

C. dimennae AY188365

C. dimennae AF410473

C. dimennae EU266559 Uncultured Fungus AM901805

$$
\begin{array}{r}
\text { T1S-3-PDA } \\
\text { Y5S-PDA }
\end{array}
$$$$
\mathrm{Y} 5 \mathrm{~S}-\mathrm{PDA}
$$

TS-1-STD

C. oeirensis AY188362

C. magnus AF190009

Y $2 \mathrm{M}-\mathrm{PDA}$

C. oeirensis AF444349

c. oeirensis AF444364

C. oeirensis EU002866

C. chernovii AF444354

C. chernovii FN400936

Uncultured fungus FM178256 U3S-PDA

Uncultured fungus AJ875389 Uncultured fungus AB520331

ltured fungus

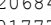
Z2S-STD

C. Carnescens EU149785 C. sp. HM849618

C. carnescens CBS8395

C. carnescens AB105438

C. victoriae AY188380

C. victoriae AJ581046

C. victoriae AM160647

C. victoriae CBS9265

C. victoriae HM849622

C. victoriae $A \mathrm{~F} 444645$

C. victorice A 442

c. victoriae AY04065 L2S-1-PDA W2S-1-PDA

C. sp. HM589279

C. victoriae AY040654 $\mathrm{S} 4 \mathrm{~S}-\mathrm{PDA}$

C. victoriae CBS9267
-AAGTCCGACC--_--ATTTCAT-_-_-_-_-_-_-TTTCTT--_-_--ACACTGTAGAAGTCCGACC----CTTTCA----T-----------TTTCTT-------ACACTGTGC AGAAGTCCGACC----CTTTCA----T---------TTTCTT------ACACTGTGC ACACCCCTGTGC-A--CTTTGGC-CACCTG--C-----CGCGCT-----TCACTGCGT-ACACC-CTGTGC-A--CTTTGGC-CACCTG--C-----CGCGCT----TA-ACTGCGTTA ACACCCCTGTGC---ACTTTGGC-CACCTG-------CCGCGCT-----TAACTGCGTTA ACACCCCTGTGC---ACTTTGGC-CACCTG-------CCGCGCT-----TAACTGCGTTA ACACCCCTGTGC---ACTTTGGC-CACCTG-------CCGCGCT-----TAACTGCGTTA АCACCCCTGTGC-A--CTTTCGC-CACCTG-C-----CGCGCT---TTACTG--CGT-

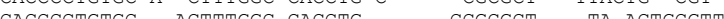

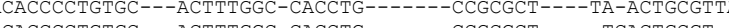

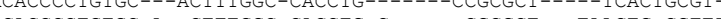
ACA-CCCTGTGC-A--CTTTGGC-CACCTG-C------CGCGCT---TAACTG-CGTTAG ACACCCCTGTGC-A--CTTTGGC-CACCTG-C------CGCGCT---TTACTG-CGTTAG ACACCCCTGTGC-A--CTTTGGC-CACCTG-C------CGCGCT---TTACTG-CGTTAG -TAACTATAAT-----CTTC---------T----------------AC-CTCTGT-AA----CTA-----T-----------------AATCTT---CTAC-СTCTGT--

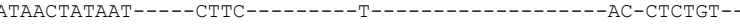

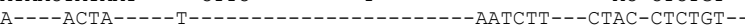
TGACCTCCGGG---T-CTTAATAACTA-----_--- TAATCTT---CTAC-CTCTGTАA--

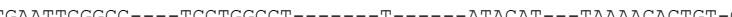
作 ---TCGGCC---T-TTTGGCCTTA-----0-TACACATAATATACA--CTGT-AATTCGGCC----TTTTGGCCTTA---T------ACACATA--ATATACACTGTTGAATTCGGCC----TTTTGGCCTTA--T--------ACACATA--ATATACACTGT-TGAATTCGGCC----TTTTGGCCTTA---T--------ACACATA--ATATACACTGT-TGAATTCGGCC----TTTTGGCCTTA---T--------ACACATA--ATATACACTGT--

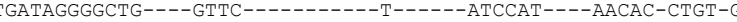
CGAAAGCCAGTG----GTTCTTCTTTCA---T------ATCCAT----AACAC-CTGTGGTCAACTGC-------TTGTCAGGCGACCCATTCATATCCAT----AACAC-CTGT-GACTTCGGTC--OCCCCTCATTCA-O-T--O-

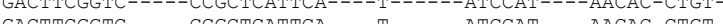

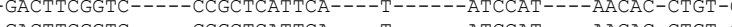
GACTTCGGIC----CCGCICATICA--1--A-ACCAT--AACAC-CTGTGTAACACTG-----T---TGGTAATTC---------TATCCAT----AACAC-CTGTGAACACTG----T--TGGTAATT------TATCCAT----AACAC-CTGTT-AACACTG-------TTGGTAATTC---------TATCCAT----AACAC-CTGT-GTAACACTG-------TTGGTAATTC--T-------ATCCAT----AACAC-CTGT-GTAACACTG-------TTGGTAATTC--T-------ATCCAT----AACAC-CTGT-GTAACACTG--------TTGGTAATTC--T--------ATCCAT----AACAC-CTGT-AAATGAGTGCCTT-CTCTTCG---GAGTTGGC-----CATCCAT-----ACACACCGT-TGCCTTCT--------CTTCGG---AGTTGGC-----CATCCAC-----ACACACCGTTGCCTTCT--0-0-CTTCGG--AGTTCGC-0-CATCCAT--TGCCITCT--TGCCICTC TAGTGCCTTC-I-CTICG-GAGIIGGC-- CATCCAC--ACACACCGTTGAGTGCCTTC----TCTTCG---GAGTTGGC-----CATCCAT-----ACACACCGTTGAGTGCCTTC---T-CIICG---GAGIIGGC----CATCCAT-----ACACACCGTTGAGTGCCTTC---T-CTTCG---GAGTTGGC-----CATCCAT-----ACACACCGT-AGA--TCTGCCC-TT--ATCGCAAGATGAGGG-----CTTCCAT-----ATACACCGT-G AGA--TCTGCCC-TT--ATCGCAAGATGAGGG-----CTTCCAT-----ATACACCGT-G AGA--TCTGCCC-TT--ATCGCAAGATGAGGG-----CTTCCAT-----ATACACCGT-G AGA--TCTGCTC--T-CTTCGCAAGAAGAGGGT-----TTCCAT-----ACACACCGT-AGA--TCTGCTC--T-CTTCGCAAGAAGAGGG-----TTTCCAT-----ACACACCGTAGA--TCTCCTC--T-CTTCGCAAGAAGAGGG----TTTCCAT----ACACACCGT-G

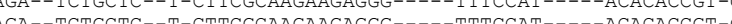

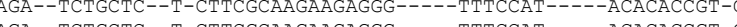
AGA-TCIGCIC-T-CITCGCAAGAAGAGGG-U-TITCCAT-U-ACACACCGTAGA--TCTGCTC--T-CTTCGCAAGAAGAGGG----1TTCCAT-----ACACACCGT--AGATCTGCTC---TCTICGCAAGAAGAGGG--1TCCAT----ACACACCGTAGA--TCTGCTC--T-CTTCGCAAGAAGAGGG-----TTTCCAT-----ACACACCGT-G AGA--TCTGCTC--T-CTTTGCAAGAAGAGGG-----TTTCCAT-----ACACACCGT-G AGA--TCTGCTC---TCTTTGCAAGAAGAGGG-----TTTCCAT-----ACACACCGTAGA--TCTGCTC--T-CTTTGCAAGAAGAGGGT-----TTCCAT-----ACACACCGT- 
R. minuta AF190010

. Slooffiae AF444552

R. slooffiae AF444627

Cy. macerans AF444317

Cy. macerans AF444329 Uncultured fungus AB520409 Uncultured fungus AB520402 Uncultured fungus AB520396 C. $\mathrm{sp} \cdot \mathrm{AF} 444448$ P4M-STD
T1S-1-PDA

C. macerans EU082232

C. macerans EU082233

C. macerans EU082231

C. macerans AY052490 Uncultured fungus EF504408

Bm. albus AF444664

B. unica AF444441

Bm. albus AB093527

Bm. albus AB093527

C. dimennae AY188365

C. dimennae AF410473 Uncultured Fungus AM901805 T1S-3-PDA Y 5 S-PDA 24S-1-STD

C. oeirensis AY188362 C. magnus AF190009 Y $2 \mathrm{M}-\mathrm{PDA}$

C. oeirensis $\operatorname{AF} 444349$

C. oeirensis AF444364

C. oeirensis EU002866

C. chernovii AF444354

C. chernovii FN400936 Uncultured fungus FM178256 U3S-PDA Uncultured fungus AJ875389 Uncultured fungus AB520331

cured fungus Uncultured fungus BJ820684

Z2S-STD

C. Carnescens EU149785 C. sp. HM849618

C. carnescens CBS8395 C. carnescens AB105438 C. victoriae AY188380 C. victoriae AJ581046 C. victoriae AM160647 C. victoriae CBS9265 C. victoriae HM84962 C. victoriae $\mathrm{A} 49622$ C. victor

c. victoriae AY04065 L2S-1-PDA L2S-1-PDA
W5S-4-STD C. sp. HM589279 c. victoriae AY040654
S4S-PDA

C. victoriae CBS9267

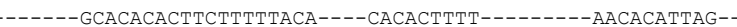
-A------CACACT-----TCTTTTTTACACACACTTTT--------AACACCTTAG--A-----CACACT----TCTTTTTTACACACACTTTT--------AACACCTTAGT------AGTAGGTGTGTCTTT-------ATAATTATACCCATATACAC--AAG-G------TAG-GTGTGTCTCTTT--------ATTTATACCCA--TATACAC--AAG-------GTAG-GTGTGTCTCTTT--A------ATT---ATACCCATATACAC--AAG------GTAG-GTGTGTCTCTTT---------ATTTATACCC----ATATACACAAG-------GTAG-GTGTGTCTCTTT---------ATTTATACCC----ATATACACAAG--G--0 --1-0AG-GTGTGTCTCTTT-------ATTTATACCC----ATATACACAAG----TAG-GTGTGTCTTTTT----AACTATA----CAC---TATATACACAAG----1AG-GTGTGTCTTTTT----AACTATA----CAC---TATATACACAAG------GAACCGTTGG--TCTTCGGA---CCGATTCTTATAA---CAAACATCTGTG-------GAACCGTTGG--TCTTCGGA---CCGATTCTTATAA---CAAACATCTGTG-------GAACCGTTGG--TCTTCGGA---CCGATTCTTATAA---CAAACATCTGTG-------GAACCGTTGG--TCTTCGG---ACCGATTCTTATAA---CAAACATCTGTG-------GAACCGTTGG--TCTTCGGA---CCGATTCTTATAA---CAAACATCTGTG-- - - GAACCGTTGG--TCTTCGGA--CCGATTCTTATAR--CARACATCTGTG-AACCGTG----GCTTCGG---CCCA----_AAC-TTGAAG-1-AACCGTG---GCTTCGG---CCCA--1--1AAC-TTGAAG-----GAACCGTG----GCTTCGG----CCCA-----AAC--------TTGAAG------GAACCGTG----GCTTCGGC----CCA------AAC--------TTGAAG-------GAACCGTG----GCTTCGGC----CCA-----AAC--------TTGAAG-----CACTGTTGGATGCTTGCA----TCCACTTTTAAAC---TAAACATTATTG--------CACTGTTGGATGCTTGCA----TCCACTTTTAAAC---TAAACATTATTG--------GCACTGTTGGATGCTTGCAT----CCACTTTTAAAC---TAAACATTATTG-----GAACTGTTGGATGCTTGCAT----CCACTTTTATAC---TAAACATTATTG作 -G------AACTGTTGGATGCTTGCATC----CACTTTTATAC---TAAACATTATTG-------GAACTGTTGGATGCTTGCAT----CCACTTTTATAC---TAAACATTATTG-------GAACTGTTGGATGCTTGCAT----CCACTTTTATAC---TAAACATTATTG-------GAACTGTTGGATGCTTGCAT----CCACTTTTATAC---TAAACATTATTG-------GAACTGTG----GCTTCGGC-----CAT-----CAC----AAAC-TGTTAG------GAACTGTG----GCTTCGGC-----CAT-----CAC----AAAC-TGTTAG-

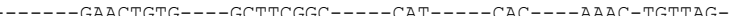
----AACTGTG----GCTTCGG-----CCATC----AC----AAAC-TGTTAG-----GAACTGTG----GCTTCGGC----CAT-----CAC----AAAC-TGTTAG------AACTGTG----GCTTCGG-----CCATC-----AC----AAAC-TGTTAG-------AACTGTG----GCTTCGG-----CCATC-----AC----AAAC-TGTTAG-------AACTGTG----GCTTCGG-----CCATC-----AC----AAAC-TGTTAG-G------AACTGTGG----CTTTGG-----CCAT-----CACA----AAC-TGTTAG--------AACTGTG----GCTTCGG-----CCATC-----AC----AAAC-TGTTAG---1--AACTGTG---GCTTCGG--_--CCATC----AC----AAAC-TGTTAG-G-1-AACTGTG---GCITCGG----CCAT----CAC---AAAC-TGTTAG---GAACTGTG---GCTTCGGC----CAT---CAC---AAAC-TGTTAG----AACTGTG----GCTTCGG----CCATC----AC----AAAC-TGTTAG-----AACTGTG----GCTTCGG-----CCATC-----AC----AAAC-TGTTAG------GAACTGTGG----CTTCGG-----CCAT-----CAC----AAAC-TGTTAGTG-------AACTGTGG----CTTCGG-----CCAT-----CACA----AAC-TGTTAG- 
R. minuta AF190010 R. slooffice AF444552 R. slooffiae AF444627 Cy. macerans AF444317

Cy. macerans AF444329 Uncultured fungus AB520409 Uncultured fungus AB520402 Uncultured fungus AB520396 C. sp. $A F 444448$

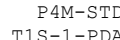

C. macerans EU082232

C. macerans EU082233

C. macerans EU082231

C. macerans AY052490 Uncultured fungus EF504408

Bm. albus AF444664 $\mathrm{V} 5 \mathrm{M}-\mathrm{PDA}$

B. unica AF444441

Bm. albus AB093527

$\mathrm{Bm}$. albus CBS7856

C. dimennae AY188365

C. dimennae AF410473

C. dimennae EU266559 Uncultured Fungus AM901805 T1S-3-PDA Y $5 \mathrm{~S}-\mathrm{PDA}$ Z4S-1-STD

C. oeirensis AY188362 C. magnus AF190009 Y $2 \mathrm{M}-\mathrm{PDA}$

C. oeirensis $\operatorname{AF} 444349$ c. oeirensis AF444364 C. oeirensis EU002866

C. chernovii AF444354

C. chernovii AF444354 Uncultured fungus FM178256 U3S-PDA

Uncultured fungus AJ875389 Uncultured fungus AB520331

lured fungus Uncultured fun Z2S-STD

C. Carnescens EU149785 C. sp. HM849618 C. carnescens CBS8395 C. carnescens AB105438 C. victoriae AY188380 C. victoriae AJ581046 C. victoriae AM160647 C. victoriae CBS9265 C. victoriae HM84962 C. victoriae $A 5444645$ C. victoriae A 444645

c. victoriae AY04065 L2S-1-PDA W5S- 2 S-STD C. $\mathrm{sp}$. HM589279 c. victoriae AY040654
S4S-PDA

C. victoriae CBS9267
TATAMGA TATAGA-ATGTA---ATAGTCTCTTAATTGAGCATAAATAAAA--ACAAAACTTTCAG TCATTGA-ATG-T----A AAATCG-T-----TATAATAAA--ACAAAACTTTCAG -TCATTGA-ATG-T----AAAATCG-T-----TATAAACTA----ATATAACTTTCAA -TCATTGA-ATG-T-----AAAATCG-------TTATAAACTA----ATATAACTTTCAA -TCATTGA-ATG-T-----AAAATCG-------TTATAAACTA----ATATAACTTTCAA -TCATTGA-ATG-T-----AAAATCG-------TTATAAACTA----ATATAACTTTCAA -TCATTGA-ATG-T----AARATCGT---0- TATAAACTA----АTATARCTTTCA CCTA TCATTGA-T

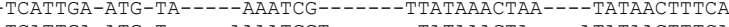
TCATTGA-ATG-T-D-AAAATCGI--D-DATAAACTA---ATATAACTTTCA TCATTGA-ATG-T-----AAAATCGT------TATAAACTA----ATATAACTTTCAA -TCATTGA-ATG-T-----AAAATCGT-------TATAAACTA----ATATAACTTTCAA -TCATTGA-ATG-T-----AAAATCGT------TATAAACTA----ATATAACTTTCAA -TA-ATGA-ACG-T-----AACCT--------ATATTAATCATA---TAAAACTTTTAA -TA-ATGA-ACG-T-----AACCT--------ATATTAATCAT---ATAAAACTTTTAA -TA-ATGA-ACG-T-----AACCT---------ATATTAATCATA---TAAAACTTTTA -TA-ATGA-ACG-T-----AACCT--------ATATTAATCAT---ATAAAACTTTTAA

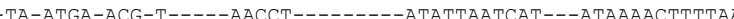
-TA-ATCA-ACG-TTA-TCTA-GA-ATG-T---ACAT-A-ATACACTTTA TC-TCGA-ATG-T-U-AATAT-A- -TCT-CGA-ATG-T-U--AATAT-A-A-U-TCAT-AACAAA-O-ATACAACTTTTA -TC-TTGA-ATG-T----AATAT------ATCAT-AACAAAA---TACAACTTTTAA -TC-TTGA-ATG-TA-----ATAT-------ATCAT-AACAAA---ATACAACTTTTAA -TC-TTGA-ATG-T-----AATAT-------ATCAT-AACAAAA---TACAACTTTTAA -TC-TTGA-ATG-T-----AATAT-------ATCAT-AACAAAA---TACAACTTTTAA -- TAACAAATG-T-----AGTCTT-A-----TTAT-AACATA---ATAAAACTTTCAA ---TAACAAATG-T-----AGTCTT-A------TTAT-AACATA---ATAAAACTTTCA

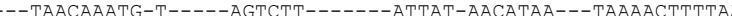
- TABCAAATG-T--o-0 - TARACAa - TARCAAATG-T-u--TAACAAATG-T---AGTCTI-A-0-TTAT-AACATA--ATAAAACTTTCA --TAACAAATG-T---AGTCTT------ATTAT-AACATA--ATAAAACTTTTA ---TAACAAATGTG------GTCTT-------ATTAT-AACTA----ATAAAACTTTTAA ---TAACAAATG-T-----AGTCTT------ATTAT-AACATAA---TAAAACTTTTAA ---TAACAAATG-T-----AGTCTT-------ATTAT-AACTA----ATAAAACTTTTAA -- TAACAAATG-T-----AGTCTT-------ATTAT-AACTA----ATAAAACTTTTAA -TA-ATGA-ATG-T-----AATAT---------CAT-AACAAAA--ACAAAACTTTTAA -TA-ATGA-ATG-T-----AАTAT---------CAT-AACAAAA--ACAАAАCTTTTA TA-ATGA-ATG-T--0-AATAT--0--0-CAT-AACABAB--ACABAACTTTTAA

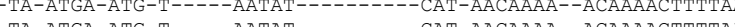
-TA-ATGA-ATG-T---AATAT--0-0-CAT-AACAMAA-ACAAAACTTTTA -TA-ATGA-ATG-T-2-AATAT-0-1-CAT-AACAAAA--ACAAAACTTTTA -TA-ATGA-ATG-T----AATAT--C-------AT-AACAAAA--ACAAAACTTTTA -TA-ATGA-ATG-T-----AATAT--------CAT-AACAAAA--ACAAAACTTTTAA -TA-ATGA-ATG-T-----AATAT---------CAT-AACAAAA--ACAAAACTTTTAA -TA-ATGA-ATG-T-----AATAT--C-------AT-AACAAAA--ACAAAACTTTTAA -TA-ATGA-ATG-T-----AATAT--C-------AT-GACAAAA--ACAAAACTTTTAA -TA-ATGA-ATG-T----AATAT--C-------AT-AACAAAA--ACAAAACTTTTAA -TA-ATGA-ATG-T-----AATAT---C------AT-AACAAAA--ACAAAACTTTTAA -TA-ATGA-ATG-T-----AATAT--C--------AT-AACAAAA--ACAAAACTTTTA

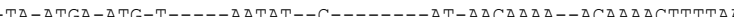
TA -TA-ATGA-ATG-T---AATAT-C--0--AT-AACAAAA--ACAAAACTTTTA -TA-TA-ATGA-ATG-TA----ATAT---C----AT-AACAAAA--ACAAAACTTTTA -TA-ATGA-ATG-T----AATAT---0----CAT-AACAAAA-TA-ATGA-ATG-T----AATAT--C------AT-AACAAAA--ACAAAACTTTTAA -TA-ATGA-ATG-T-----AATAT--C-------AT-AACAAAA--ACAAAACTTTTAA -TA-ATGA-ATG-T-----AATAT---C-------AT-AACAAAA--ACAAAACTTTTAA 
R. minuta AF190010 R. Slooffiae AF444552 R. slooffiae AF444627 Cy. macerans AF444317

Cy. macerans AF444329 Uncultured fungus AB520409 Uncultured fungus AB520402 Uncultured fungus AB520396

C. sp. $A F 444448$ P4M-STD
T1S-1-PDA

C. macerans EU082232

C. macerans EU082233

C. macerans EU082231

C. macerans AY052490 Uncultured fungus EF504408

$\mathrm{Bm}$. albus $\mathrm{AF} 444664$ $\mathrm{V} 5 \mathrm{M}-\mathrm{PDA}$

B. unica AF444441

Bm. albus AB093527

Bm. albus CBS7856

C. dimennae AY188365

C. dimennae AF410473

C. dimennae EU266559 Uncultured Fungus AM901805 T1S-3-PDA Y $5 \mathrm{~S}-\mathrm{PDA}$ Z4S-1-STD

C. oeirensis AY188362

C. magnus AF190009

Y $2 \mathrm{M}-\mathrm{PDA}$

C. oeirensis $\mathrm{AF} 444349$

C. oeirensis AF444346

C. oeirensis EU002866

C. chernovii AF44435

C. chernovii FN400936

Uncultured fungus FM178256 $\mathrm{J} 3 \mathrm{~S}-\mathrm{PDA}$

Uncultured fungus AJ875389 Uncultured fungus AB520331

-1
-1 Uncultured fungus B J820684

Z2S-ST

C. sp. HM 849618

C. carnescens CBS8395

C. carnescens AB105438

C. victoriae AY188380

C. victoriae AJ581046

C. victoriae AM160647

C. victoriae CBS9265

C. victoriae HM849622

c. victoriae

C. victoriae AF 444645

C. victoriae AY0 4065 L2S-1-PDA

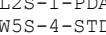

C. sp. HM589279

C. victoriae AY040654 $\mathrm{S} 4 \mathrm{~S}-\mathrm{PDA}$

C. victoriae CBS9267
CAACGGATCTCTTGGCTCTCGCATCGATGAAGAACGCAGCGAATTGCGATAAGTAATGTG

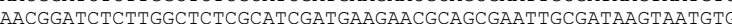
CAACGGATCTCTTGGCTCTCGCATCGATGAAGAACGCAGCGAATTGCGATAAGTAATGTG CAACGGATCTCTTGGTTCTCGCATCGATGAAGAACGCAGCGAATTGCGATAAGTAATGT CAACGGATCTCTTGGTTCTCGCATCGATGAAGAACGCAGCGAATTGCGATAAGTAATGT CAACGGATCTCTTGGTTCTCGCATCGATGAAGAACGCAGCGAATTGCGATAAGTAATGTG CAACGGATCTCTTGGTTCTCGCATCGATGAAGAACGCAGCGAATTGCGATAAGTAATGT CAACGGATCTCTTGGTTCTCGCATCGATGAAGAACGCAGCGAATTGCGATAAGTAATGTG CAACGCATCTCTTGGTTCTCGCATCGATGAHGAACGCAGCGATTGCGATAAGTAATGTG CARCGG

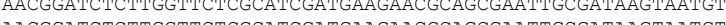
AACGGATCTCTTGGTTCTCGCATCGATAAGAACCCAGCGATTGCGATAAGTAATGT AAACGATCTCTTGGTTCTCGCATCGATGAAGAACGCAGCGAATTGCGATAAGTAATGTG CAACGGATCTCTTGGTTCTCGCATCGATGAAGAACGCAGCGAATTGCGATAAGTAATGTG CAACGGATCTCTTGGTTCTCGCATCGATGAAGAACGCAGCGAATTGCGATAAGTAATGTC CAACGGATCTCTTGGCTCTCGCATCGATGAAGAACGCAGCGAAATGCGATAAGTAATGT CAACGGATCTCTTGGCTCTCGCATCGATGAAGAACGCAGCGAAATGCGATAAGTAATGTG CAACGGATCTCTTGGCTCTCGCATCGATGAAGAACGCAGCGAAATGCGATAAGTAATGTC CAACGGATCTCTTGGCTCTCGCATCGATGAAGAACGCAGCGAAATGCGATAAGTAATGTC CAACGCATCTCTTCGCTCTCCCATCGATCAAGAACCCAGCGAA

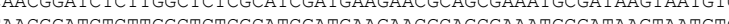
CAACGATCTCTTGGCTCTCGCATCGATGAAGAACGCAGCGAAATGCGATAAGTAATGTG CAACGGATCTCTTGGCTCTCGCATCGATGAAGAACGCAGCGAAATGCGATAAGTAATGTG CAACGGATCTC CAACGGATCTCTTGGCTCTCGCATCGATGAAGAACGCAGCGAAATGCGATAAGTAATGT CAACGGATCTCTTGGCTCTCGCATCGATGAAGAACGCAGCGAAATGCGATAAGTAATGTG CAACGGATCTCTTGGCTCTCGCATCGATGAAGAACGCAGCGAAATGCGATAAGTAATGTC CAACGGATCTCTTGGCTCTCGCATCGATGAAGAACGCAGCGAAATGCGATAAGTAATGT CAACGGATCTCTTGGCTCTCGCATCGATGAAGAACGCAGCGAAATGCGATAAGTAATGTG CAACGGATCTCTTGGCTCTCGCATCGATGAAGAACGCAGCGAAATGCGATAAGTAATGTG CAACGGATCTCTTGGCTCTCGCATCGATGAAGAACGCAGCGAAATGCGATAAGTAATGTG CARCGCATCTCTTGCCTCTCCCATCGATGABGAACGCAGCGABATGCGATAAGTAATGTG CTCTTGGCTCTCGCATCGATGAAGAACGCAGC CAACGGATCTCTTGGCTCTCGCATCGATGAAGAACGCAGCGAAATGCGATAAGTAATGT CAACGGATCTCTTGGCTCTCGCATCGATGAAGAACGCAGCGAAATGCGATAAGTAATGT

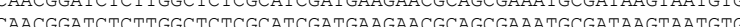
CAACGGATCTCTTGGCTCTCGCATCGATGAAGAACGCAGCGAAATGCGATAAGTAATGTC CAACGGATCTCTTGGCTCTCGCATCGATGAAGAACGCAGCGAAATGCGATAAGTAATGT CAACGGATCTCTTGGCTCTCGCATCGATGAAGAACGCAGCGAAATGCGATAAGTAATGT CAACGGATCTCTTGGCTCTCGCATCGATGAAGAACGCAGCGAAATGCGATAAGTAATGT CAACGGATCTCTTGGCTCTCGCATCGATGAAGAACGCAGCGAAATGCGATAAGTAATGTC CAACGGATCTCTTGGCTCTCGCATCGATGAAGAACGCAGCGAAATGCGATA CABCGGATCTCTTGGCTCTCGCATCGATGABGAACGCAGCGABATGCGATAAGTARTGTG 作 CAACGGATCTCTTGGCTCTCGCATCGATGAAGAA CAACGGATCTCTTGGCTCTCGCATCGATGAAG CAACGGATCTCTTGGCTCTCGCATCGATGAAGAACGCAGCGAAATGCGATAAGTAATGT CAACGGATCTCTTGGCTCTCGCATCGATGAAGAACGCAGCGAAATGCGATAAGTAATGTG CAACGGATCTCTTGGCTCTCGCATCGATGAAGAACGCAGCGAAATGCGATAAGTAATGTG CAACGGATCTCTTGGCTCTCGCATCGATGAAGAACGCAGCGAAATGCGATAAGTAATGT CAACGGATCTCTTGGCTCTCGCATCGATGAAGAACGCAGCGAAATGCGATAAGTAATGTG CAACGGATCTCTTGGCTCTCGCATCGATGAAGAACGCAGCGAAATGCGATAAGTAATGTG CAACGGATCTCTTGGCTCTCGCATCGATGAAGAACGCAGCGAAATGCGATAAGTAATGTG CAACCATCTCTTGCCTCTCGCATCGATGARGAACCCAGCGAAATGCGATAAGTAATGT 作 CAACGGACTCINGGCICICGCAICGATGAAGACGCAGCGAAATGCGATAAGTAATGT CAACGGATCTCTTGGCTCTCGCATCGATGAAGAACGCAGCGAAATGCGATAAGTAATGT CAACGGATCTCTIGGCTCTCGCATCGATGAAGAACGCAGCGAAATGCGATAAGTAATGT CAACGGATCTCTTGGCTCTCGCATCGATGAAGAACGCAGCGAAATGCGATAAGTAATGTG CAACGGATCTCTTGGCTCTCGCATCGATGAAGAACGCAGCGAAATGCGATAAGTAATGTG CAACGGATCTCTTGGCTCTCGCATCGATGAAGAACGCAGCGAAATGCGATAAGTAATGTG CAACGGATCTCTTGGCTCTCGCATCGATGAAGAACGCAGCGAAATGCGATAAGTAATGT 
R. minuta AF190010 R. Slooffiae AF 444552 R. slooffiae AF444627

Cy. macerans AF444317

Cy. macerans AF44432 Uncultured fungus AB520409 Uncultured fungus AB520402 Uncultured fungus AB520396

C. sp. $A F 444448$ P4M-STD
T1S-1-PDA

C. macerans EU082232

C. macerans EU082233

C. macerans EU082231

C. macerans AY052490 Uncultured fungus EF504408

Bm. albus AF444664 $\mathrm{V} 5 \mathrm{M}-\mathrm{PDA}$

B. unica AF444441

Bm. albus AB093527

Bm. albus CBS7856

C. dimennae AY188365

C. dimennae AF410473

C. dimennae EU266559 Uncultured Fungus AM901805 T1S-3-PDA $Y 5 S-P D A$ Z4S-1-STD

C. oeirensis AY188362

C. magnus AF190009

Y $2 \mathrm{M}-\mathrm{PDA}$

C. oeirensis AF444349

c. oeirensis AF444364

C. oeirensis AF 444364

C. chernovii AF444354

C. chernovii AF444354

Uncultured fungus FM178256 U3S-PDA

Uncultured fungus AJ875389 Uncultured fungus AB520331

-1
-1 Uncultured fungus

Z2S-ST

C. sp. HM849618

C. carnescens CBS8395

C. carnescens AB105438

C. victoriae AY188380

C. victoriae AJ581046

C. victoriae AM160647

C. victoriae CBS9265

C. victoriae HM849622

C. victoriae AF444645

C. victoriae AY040656

C. victoriae AJ581048 L2S-1-PDA TSS-4-STD

C. sp. HM589279

C. victoriae AY040654

C. victoriae CBS9267
AATTGCAGAATTCAGTGAATCATCGAATCTTTGAACGCACCTTGCACTCTTT-GGTATTC

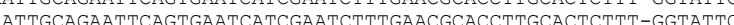
AATTGCAATTCAGTGAATCATCGAATCTTTGAACGCACCTTGCACTCTTT-GGTATTC

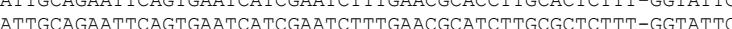
作 AATTGCAGAATTCAGTGAATCATCGAATCTTTGAACGCATCTTGCGCTCTTT-GGTATTC AATTGCAGAATTCAGTGAATCATCGAATCTTTGAACGCATCTTGCGCTCTTT-GGTATTC AATTGCAGAATTCAGTGAATCATCGAATCTTTGAACGCATCTTGCGCTCTTT-GGTATTC ATTCCACAATCACTC MTCOCAGATC AATTGCAGAATTCAGTGAATCATCGAATCTTTGAR AATTGCAGAATTCAGTGAATCATCGAATCTTTGAACGCATCTTGCGCTCTTT-GGTATT AATTGCAGAATTCAGTGAATCATCGAATCTTTGAACGCATCTTGCGCTCTTT-GGTATTC AATTGCAGAATTCAGTGAATCATCGAATCTTTGAACGCATCTTGCGCTCTTT-GGTATTC AATTGCAGAATTCAGTGAATCATCGAATCTTTGAACGCACCTTGCGC-CTTTTGGTATTC AATTGCAGAATTCAGTGAATCATCGAATCTTTGAACGCACCTTGCGC-CTTTTGGTATTC AATTGCAGAATTCAGTGAATCATCGAATCTTTGAACGCACCTTGCGC-CTTTTGGTATTC AATTGCAGAATTCAGTGAATCATCGAATCTTTGAACGCACCTTGCGC-CTTTTGGTATTC ATTCCACAATCAGTGAACATCGATCIC TATCCAGAAR ATTGCAGAATTCAGTGAATCATCGAATCTTTGAACGCACCTTGCGCCCTCI-C AATTGCAGAATTCAGTGAATCATCGAATCTTTGAACGCACCTTGCGCCCTCT-GGTATTC AATTGCAGAATTCAGTGAATCATCGAATCTTTGAACGCACCTTGCGCCCTCT-GGTATTC AATTGCAGAATTCAGTGAATCATCGAATCTTTGAACGCACCTTGCGCCCTCT-GGTATTC AATTGCAGAATTCAGTGAATCATCGAATCTTTGAACGCACCTTGCGCCCTCT-GGTATTC AATTGCAGAATTCAGTGAATCATCGAATCTTTGAACGCACCTTGCGCCCTCT-GGTATTC AATTGCAGAATTCAGTGAATCATCGAATCTTTGAACGCACCTTGCGCCCTCT-GGTATTC AATTGCAGAATTCAGTGAATCATCGAATCTTTGAACGCACCTTGCGCTCCTT-GGTATTC AATTGCAGAATTCAGTGAATCATCGAATCTTTGAACGCACCTTGCGCTCCTT-GGTATTC AATTGCAGAATTCAGTGAATCATCGAATCTTTGAACGCACCTTGCGCTCCTT-CGTATTC

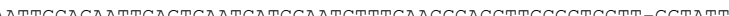
ATTCCACAATTCAGTGAATCATCGAATCTTTGAACGCACCTTCCGCTC AATTGCAGAATTCAGTGAATCATCGAATCTTTGAACGCACCTTGCGCTCCTT-GGTATTC ATTGCACAATCAGTGAATCATCGAATTCTTTGAACGCACCTTGCGCTCCTT-GGTATTC 作 AATTGCAGAATTCAGTGAATCATCGAATCTTTGAACGCACCTTGCGCTCCTT-GGTATTC AATTGCAGAATTCAGTGAATCATCGAATCTTTGAACGCACCTTGCGCTCCTT-GGTATTC AATTGCAGAATTCAGTGAATCATCGAATCTTTGAACGCACCTTGCGCTCCTT-GGTATTC AATTGCAGAATTCAGTGAATCATCGAATCTTTGAACGCACCTTGCGCTCCTT-GGTATTC AATTGCAGAATTCAGTGAATCATCGAATCTTTGAACGCACCTTGCGCCCTTT-GGTATTC AATTGCAGAATTCAGTGAATCATCGAATCTTTGAACGCACCTTGCGCCCTTT-GGTATTC A AATTGCAGAATTCAGTGAATCATCGAATCTTTGAACGCACCII AATTGCAGAATTCAGTGAATCATCGAATCTTTGAACGCACCTTGCGCCCTTT-GGTATTC AATIGCAGAATTCAGTGAATCATCGAATCTTTGAACGCACCTIGCGCCCTIT-GGTATT AATTGCAGAATTCAGTGAATCATCGAATCTTTGAACGCACCTTGCGCCCTTT-GGTATTC AATTGCAGAATTCAGTGAATCATCGAATCTTTGAACGCACCTTGCGCCCTTT-GGTATTC AATTGCAGAATTCAGTGAATCATCGAATCTTTGAACGCACCTTGCGCCCTTT-GGTATTC AATTGCAGAATTCAGTGAATCATCGAATCTTTGAACGCACCTTGCGCCCTTT-GGTATTC AATTGCAGAATTCAGTGAATCATCGAATCTTTGAACGCACCTTGCGCCCTTT-GGTATTC AATTGCAGAATTCAGTGAATCATCGAATCTTTGAACGCACCTTGCGCCCTTT-GGTATTC AATTCCACAATCACTCAATCATCGAATCTTTGAACGCACCTTGCGCCCTTT-GCTATTC

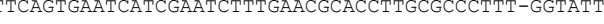
ATTGCAGAATTCAGTGAATCATCGAATCTTTGAACGCACCTTGCGCCCTTT-GGTATT ATTGCAGAATTCAGTGAATCATCGAATCTTTGAACGCACCTTGCGCCCTTT-GGTATTC AATTGCAGAATTCAGTGAATCATCGAATCTTTGAACGCACCTTGCGCCCTTT-GGTATTC AATTGCAGAATTCAGTGAATCATCGAATCTTTGAACGCACCTTGCGCCCTTT-GGTATTC AATTGCAGAATTCAGTGAATCATCGAATCTTTGAACGCACCTTGCGCCCTTT-GGTATTC AATTGCAGAATTCAGTGAATCATCGAATCTTTGAACGCACCTTGCGCCCTTT-GGTATTC 
R. minuta AF190010 R. slooffiae AF444552 R. slooffiae AF444627 Cy. macerans AF444317 Cy. macerans AF444329 Uncultured fungus AB520409 Uncultured fungus AB520402 Uncultured fungus AB520396

C. sp. AF444448 P4M-STD
T1S-1-PDA

C. macerans EU082232

C. macerans EU082233

C. macerans EU082231

C. macerans AY052490 Uncultured fungus EF50440

Bm. albus AF444664 $\mathrm{V} 5 \mathrm{M}-\mathrm{PDA}$

B. unica AF444441

$\mathrm{Bm}$. albus AB093527

Bm. albus CBS7856

C. dimennae AY188365

C. dimennae AF410473

C. dimennae EU266559 Uncultured Fungus AM901805 T1S-3-PDA $Y 5 S-P D A$ Z4S-1-STD

C. oeirensis AY188362 C. magnus AF190009 Y $2 \mathrm{M}-\mathrm{PDA}$

C. oeirensis AF444349

C. oeirensis AF444364

C. oeirensis AF444364

C. chernovii AF444354

C. chernovii AF444354 Uncultured fungus FM178256 U3S-PDA

Uncultured fungus AJ875389 Uncultured fungus AB520331

C. carnescens AB03505 Uncultured fungus FJ82068 Uncultured fungus AM901777

C. carnescens EU149785

C. sp. HM849618

C. carnescens CBS8395

C. carnescens AB105438

C. victoriae AY188380

C. victoriae AJ581046

C. victoriae AM160647

C. victoriae CBS9265

C. victoriae HM849622

C. victoriae AF444645

C. victoriae AY040656

C. victoriae AJ581048 L2S-1-PDA $5 S-4-S T D$

C. sp. HM589279

C. victoriae AY040654

C. victoriae CBS9267
CGAAGAGTATGTCTGTTTGAGTATCATGAAACTCTCAACC---CCCC--TATTTTGTAAT CGAAGAGTATGTCTGTTTGAGTGTCATGAAACTCTC-AAC---CCCCCTATTTTGTAATG CGAAGAGTATGTCTGTTTGAGTGTCATGAAACTCTC-AAC---CCCCCTATTTTGTAATG CGAAGAGCATGCCTGTTTGAGTGTCATGAAACTCTC--AC---CTCCAGCCTTCTTT-AA CGAAGAGCATGCCTGTTTGAGTGTCATGAAACTCTC--AC---CTCCAGCCTTCTTT-AA CGAAGAGCATGCCTGTTTGAGTGTCATGAAACTCTC--AC---CTCCAGCCTTCTTT-AA CGAAGAGCATGCCTGTTTGAGTGTCATGAAACTCTC--AC---CTCCAGCCTTCTTT-A CGAAGAGCATGCCTGTTTGAGTGTCATGAAACTCTC--AC---CTCCAGCCTTCTTT-A CAAGACCATGCCTCTTTCAGTCTCATGAAACTCTC--AC---CTCCAGCCTTCTTT-AA COACA CGAAGACATCCTGT

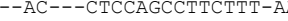
CGAAGAGCATGCCTGTTTGAGTGTCATGAAACTCTC--AC---CTCCAGCCTTCTTT-AA CGAAGAGCATGCCTGTTTGAGTGTCATGAAACTCTC--AC---CTCCAGCCTTCTTT-AA CGAAGAGCATGCCTGTTTGAGTGTCATGAAACTCTC--AC---CTCCAGCCTTCTTT-AA CGAAAGGCATGCCTGTTTGAGTGTCATGAA-ATATC-AAC---CCC---TCCTGGTT-TC CGAAAGGCATGCCTGTTTGAGTGTCATGAA-ATATC-AAC---CCC---TCCTGGTT-TC CGAAAGGCATGCCTGTTTGAGTGTCATGAA-ATATC-AAC---CCC---TCCTGGTT-TC CGAAAGGCATGCCTGTTTGAGTGTCATGAA-ATATC-AAC---CCC---TCCTGGTT-TC CGAAACCATCCCTGTTTGAGTGTCATGAA-ATATC-AAC---CCC---TCCTGGTT-TC CGAAAGCATCCTTGTTCAGTGTCATGAA-ATATC-AAC--CCC-CGGAGGGCATGCCTGTTTGAGTGTCATGAACACCTC--AC-CGGAGGGCATGCCTGTTTGAGTGTCATGAACACCTC--AC---CCC----CTCGGGT-TI CGGAGGGCATGCCTGTTTGAGTGTCATGAACACCTC--AC---CCC----CTCGGGT-T CGGAGGGCATGCCTGTTTGAGTGTCATGAACACCTC--AC----CCC----CTCGGGTTTC CGGAGGGCATGCCTGTTTGAGTGTCATGAACACCTC--ACC--CCC-----TCGGGTTTC CGGAGGGCATGCCTGTTTGAGTGTCATGAACACCTC--AC---CCC----CTCGGGTTTC CGGAGGGCATGCCTGTTTGAGTGTCATGAACACCTC--AC---CCC----CTCGGGTTTC CGAGGAGCATGCCTGTTTGAGTGTCATGAAACCCTCAAAC---C-CAAGTTTTGGAT-TT CGAGGAGCATGCCTGTTTGAGTGTCATGAAACCCTCAAAC---C-CAAGTTTTGGAT-TI CGAGGAGCATGCCTGTTTGAGTGTCATGAAACCCTCAAA----CCCAAGTTTTGGAT-TT CGAGGAGCATGCCTGTTTGAGTGTCATGAAACCCTCAAAC--C-CAAGTTTTGCAT-TI CGACATCAT-T

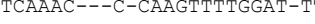
CGAGGAGCATGCCTGTTTGAGTGTCATGAAACCCTCAAAC---C-CAAGTTTTGGAT-TI CGAGGAGCATGCCTGTTTGAGTGTCATGAAACTCTCAAAC---C-CAAGTTTTGGAT-TT

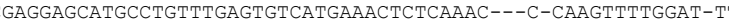
CGAGGAGCATGCCTGTTTGAGTGTCATGAAACTCTCAAA----CCCAAGTTTTGGAT-TT CGAGGAGCATGCCTGTTTGAGTGTCATGAAACTCTCAAA----CCCAAGTTTTGGAT-T' CGAGGAGCATGCCTGTTTGAGTGTCATGAAACTCTCAAA----CCCAAGTTTTGGAT-TI CGAGGAGCATGCCTGTTTGAGTGTCATGAAACTCTCAAA----CCCAAGTTTTGGAT-TT CGAAGGGCATGCCTGTTTGAGTGTCATGAA-ACCTC--AC---CCCA---CTTGGGT-TT CGAAGGGCATGCCTGTTTGAGTGTCATGAA-ACCTC--AC---CCCA---CTTGGGT-TT CCAACCATCCCTGTTTGAGTGTCATGAA-ACCTC--AC--CCCA--CTTGGGT-TT CAAGGCATGCCIGTITGAGTGICATGAA-ACCTC--AC---CCA---CTIGGGT-T CGAAGGGCATGCCTGITTGAGGICAGA-ACCIC--AC--CCCA--CITGGGT-T CGAAGGCCATGCCTGTTTGAGTGTCATGAA-ACCTC--AC--CCCA---CTTGGGT-TT CGAAGGGCATGCCTGTTTGAGTGTCATGAA-ACCTC--AC---CCCA---CTTGGGT-TT

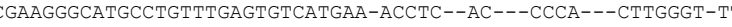
CGAAGGGCATGCCTGTTTGAGTGTCATGAA-ACCTC--AC---CCCA---CTTGGGT-TT CGAAGGGCATGCCTGTTTGAGTGTCATGAA-ACCTC--AC---CCCA---CTTAGGT-TT CGAAGGGCATGCCTGTTTGAGTGTCATGAA-ACCTC--AC---CCCA---CTTAGGT-TI CGAAGGGCATGCCTGTTTGAGTGTCATGAA-ACCTC--AC---CCCA---CTTAGGT-TT CGAAGGGCATGCCTGTTTGAGTGTCATGAA-ACCTC--AC---CCCA---CTTGGGT-TT CGAAGGGCATGCCTGTTTGAGTGTCATGAA-ACCTC--AC---CCCA---CTTGGGT-TI CGAACCCCATCCCTGTTTCACTGTCATCAA-ACCTC--AC--CCCA--CTTCCGT-TT COACA CGAAG (1) CGAAGGGCATGCCTGTTTGAGTGTCATGAA-ACCTC--AC---CCCA---CTTGGGT-TT CGAAGGGCATGCCTGTTTGAGTGTCATGAA-ACCTC--AC---CCCA---CTTGGGT-T CGAAGGGCATGCCTGTTTGAGTGTCATGAA-ACCTC--AC---CCCA---CTTGGGT-TI CGAAGGGCATGCCTGTTTGAGTGTCATGAA-ACCTC--AC---CCCA---CTTGGGT-TI CGAAGGGCATGCCTGTTTGAGTGTCATGAA-ACCTC--AC---CCCA---CTTGGGT-T' CGAAGGGCATGCCTGTTTGAGTGTCATGAA-ACCTC--AC---CCCA---CTTGGGT-T 
R. minuta AF190010 . Slooffiae AF444552 R. slooffiae AF444627 Cy. macerans AF444317

Cy. macerans AF444329 Uncultured fungus AB520409 Uncultured fungus AB520402 Uncultured fungus AB520396

C. sp. AF444448 P4M-STD P4M-STD

C. macerans EU082232

C. macerans EU082233

C. macerans EU082231

C. macerans AY052490 Uncultured fungus EF504408

Bm. albus AF444664 $\mathrm{V} 5 \mathrm{M}-\mathrm{PDA}$

B. unica AF444441

$\mathrm{Bm}$. albus AB093527

Bm. albus CBS7856

C. dimennae AY188365

C. dimennae AF410473

C. dimennae EU266559 Uncultured Fungus AM901805 $\mathrm{T} 1 \mathrm{~S}-3-\mathrm{PDA}$
$\mathrm{Y} 5 \mathrm{~S}-\mathrm{PDA}$ Y 5 S-PDA

C. oeirensis AY188362 C. magnus AF190009 Y $2 \mathrm{M}-\mathrm{PDA}$

C. oeirensis $\operatorname{AF} 444349$

c. oeirensis AF444364

C. oeirensis AF 444364

C. chernovii AF444354

C. chernovii AF444354 Uncultured fungus FM178256 U3S-PDA

Uncultured fungus AJ875389 Uncultured fungus AB520331
C. carnescens AB035050

C. carnescens aBO3 Uncultured fungus BJ820684 Z2S-STD

C. Carnescens EU149785 C. sp. HM849618 C. carnescens CBS8395 C. carnescens AB105438 C. victoriae AY188380 C. victoriae AJ581046 C. victoriae AM160647 C. victoriae CBS9265 C. victoriae HM 849622 c. victoriae C.

C. victoriae AY0 4065 L2S-1-PDA L2S-1-PDA
W5S-4-STD

C. sp. HM589279

c. victoriae AY04065 $\mathrm{S} 4 \mathrm{~S}-\mathrm{PDA}$

C. victoriae CBS9267
--GAAATGGGCGTGGGCTTGGATTATGGTTGTCTGTCG--GCGTAATTGC------_CG AGATGGGTGT----GGGCTTGGATTATGGTTGTCTGTCG--GCGTAATTGC------CGG AGATGGGTGT----_GGATTGGATTATGGTTGTCTGTCG--GCGTAATTGC------CGG TTAGAGGTGTTG--GGGCGTGGACG-TGAGTGC-TGCTGGTGCCC---TGGTTGCATCGC TTAGAGGTGTTG--GGGCGTGGACG-TGAGTGC-TGCTGGTGCCC---TGGTTGCATCGC TTAGAGGTGTT--GGGGCGTGGACG-TGAGTGC-TGCTGGTGCCC---TGGTTGCATCGG TTAGAGGTGTT--GGGGCGTGGACG-TGAGTGC-TGCTGGTGCCC---TGGTTGCATCGC TTAGAGGTGTT--GGGGCGTGGACG-TGAGTGC-TGCTGGTGCCC---TGGTTGCATCGC TTACACGTGT--CGGCCGTCGACG-TGAGTGC-TGCTGGTGCCC---TGGTTGCATCGG TIACACA TAGAGGTGT-GGGGCGTGGACGT-GAGTGC-TGCTGGTGCCC--TGGITGCATCG TAGAGGTGTT--GGGGCGTGGACG-TGAGTGC-TGCTGGGCC TTAGAGGTGTT--GGGGCGTGGACG-TGAGTGC-TGCTGGTGCCC---TGGTTGCATCGG ITAGAGGTGTT--GGGGCGTGGACG-TGAGTGC-TGCTGGTGCCC---TGGTTGCATCGG ITAGAGGTGTT--GGGGCGTGGACG-TGAGTGC-TGCTGGTGCCC---TGGTTGCATCGG TGATCAGTGTT---GGGCTTGGACT------TGGGTGTCT--GCCAGTTCGCT-------GG TGATCAGTGTT---GGGCTTGGACT-----TGGGTGTC--TGCCAGTTCGC------TGG TGATCAGTGTT---GGGCTTGGACT------TGGGTGTCT--GCCAG--GACTTGTCCTGC TGATCAGTGTT---GGGCTTGGACT-----TGGGTGTC--TGCCAG--GACTTGTCCTGG TGATCAGTGTT---GGGCTTGGACT-----TGGGTGTC--TGCCAGCTCGC------TGG TCATCA CC--

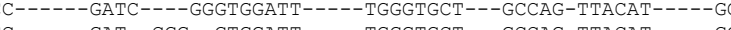
CC------GAT--CGG--GTGGATT-----TGGGTGCT---GCCAG-TTACAT-----GG CGATC----------GGGTGGATT-----TGGGTGCT---GCCAG-TTACAT-----GG CGATC------------GGGTGGATT-----TGGGTGCT---GCCAG-TTACAT------GG CGATC-----------GGGTGGATT-----TGGGTGCT---GCCAG-TTACAT-----GG CGATC-----------GGGTGGATT-----TGGGTGCT---GCCAG-TTACAT-----GG CGGTCCATGCT--TGAGTTTGGATT-----TGGATGTT--TGCCGG--TGA-TGAACCGA CGATCCATGCT--TGAGTTTGGATT-----TGGATGTT--TGCCGG--TGA-TGAACCGA CGATCCTTGCT--TGAGTTTGGATT-----TGGATGTTT--GCCGG--TGATGAAC-CGA CGTCCATGCT-TGAGTTTGCATT---OTGGACGTT--TCCCGG--TGA-TGAACCGA CGACACA (1) 作 CGATCCATGCI--TGAGTTTGGATT----TGGATGTT--TGCCGG--TGA-TAAGCCGA

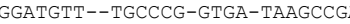
CGATCCATGCT--TGAGTTTGGATT-----TGGATGTT--TGCCGG--TGA-TAAGCCGA CGATCCATGCT--TGAGTTTGGATT-----TGGATGTTT--GCCGG--TGA-TAAGCCGA CGATCCATGCT--TGAGTTTGGATT-----TGGATGTTT--GCCGG--TGA-TAAGCCGA CGATCCATGCT--TGAGTTTGGATT-----TGGATGTTT--GCCGG--TGA-TAAGCCGA TTGCC--TGAG--CGGTGGTGTATT------GGGTGTT---GCC---TTGCCAAA---GG TTGCC--TGAG--CGGTGGTGTATT-------GGGTGTT---GCC---TTGCCAAA---GG TTCCC--TGAG--CGGTGGTGTATT-----GGGTGTT--OCC-- TTGCCAAA-- GG TIGCC--TGAG-CGGTGTA ITGCC-ITCC-TGAG-CGGTGGTGTATT-ITGCC--TGAG--CGGTGGTGTATT-----GGGTGIT---GCC---TTGCCAAA---GG TTGCC--TGAG--CGGTGGTGTATI-----_GGTGIT---GCC---TTGCCAAA---GG TTGCC--TGAG--CGGTGGTGTATT------_GGGTGTT---GCC---TTGCCAAA---GG TTGCCTAAG----CGGTGGTGTATT------_GGGTGTT---GCC---TTGCTAAA---GG TTGCCTAG-----CGGTGGTGTATT------GGGTGTT--TGCC---TTGCTAAA---GG TTGCCTAAG----CGGTGGTGTATT------GGGTGTT--TGCC---TTGCTAAA---GG TTGCC--TGAGC--GGTGGTGTATT------GGGTGTT---GCC---TTGCTAAA---GG TTGCC--TGAG--CGGTGGTGTATT------_GGGTGTT---_GCC---TTGCTAAA---GG TTGCC--TGAG-CGGTGGTGTATT-----GGGTGTT---GCC---TTGCTAAA-- GG TIGCC-TGAG-CGGTGGTGTATT-

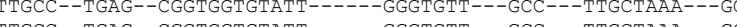

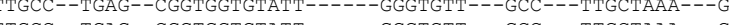
ITGCC--TGAG--CGGTGGTGTATI-----_GGGTGTT---GCC---TTGCTAAA---GG ITGCC--TGAG--CGGIGGIGIATT------GGGTGIT---GCC---TTGCTAAA---GG ITGCC--TGAG--CGGTGGTGTATT------GGGTGTT---GCC---TTGCTAAA---GG TTGCC--TGAG--CGGTGGTGTATT------GGGTGTT---GCC---TTGCTAAA---GG TTGCC--TGAG--CGGTGGTGTATT------GGGTGTT---GCC---TTGCTAAA---GG TTGCC--TGAGC--GGTGGTGTATT------_GGGTGTT---GCC---TTGCTAAA---GG 
R. minuta AF190010 R. Slooffice AF444552 R. slooffiae AF444627 Cy. macerans AF444317

Cy. macerans AF444329 Uncultured fungus AB520409 Uncultured fungus AB520402 Uncultured fungus AB520396 C. sp. AF444448 P4M-STD
T1S-1-PDA

C. macerans EU082232

C. macerans EU082233

C. macerans EU082231

C. macerans AY052490 Uncultured fungus EF504408 Bm. albus AF444664

B. unica $A F 44444$

Bm. albus AB093527

$\mathrm{Bm}$. albus CBS7856

C. dimennae AY188365

C. dimennae AF410473

C. dimennae EU266559 Uncultured Fungus AM901805 T1S-3-PDA Y 5 S-PDA Z4S-1-STD

C. oeirensis AY188362 C. magnus AF190009 Y $2 \mathrm{M}-\mathrm{PDA}$

C. oeirensis AF444349 C. oeirensis AF444364 C. oeirensis EU002866 C. chernovii AF444354 C. chernovii AF444354 Uncultured fungus FM178256 U3S-PDA

Uncultured fungus AJ875389 Uncultured fungus AB520331

lured fungus 40684 Uncultured fungus AM901777 Z2S-STD

C. carnescens EU149785 C. sp. HM849618 C. carnescens CBS8395 C. carnescens AB105438 C. victoriae AY188380 C. victoriae AJ581046 C. victoriae AM160647 C. victoriae CBS9265 C. victoriae HM849622 C. victoriae C. victoriae AF 444645

c. victoriae AY0 4065 L2S-1-PDA $\mathrm{L} 2 \mathrm{~S}-1-\mathrm{PDA}$
$\mathrm{W} 5 \mathrm{~S}-4-\mathrm{STD}$ C. sp. HM589279 c. victoriae AY040654

C. victoriae CBS9267
CTCAACTGAAATAC-ACGAGCA----_-_-ACCCTATT---GAAATAGACGGTTTGACT TCAACTGAAATAC-ACGAGCA---------ACCCTATT---GAAATAAACGGTTTGACT CTCAACTGAAATAC-ACGAGCA------ACCCTATT---GAAATAAACGGTTTGACT CTCACTTGAAATATG-TTAGCTG-A------CTCCTCTA-------GAGGTGGTTCTAC CTCACTTGAAATAC-ATTAGCTG-A------ATCCTCTA-------GAGGTGGTTCTACT CTCACTTGAAATAC-ATTAGCTGA-------ATCCTCT-------AGAGGTGGTTCTACT CTCACTTGAAATAC-ATTAGCTGA-------ATCCTCT-------AGAGGTGGTTCTACT CTCACTTGAAATAC-ATTAGCTGA-------ATCCTCT-------AGAGGTGGTTCTACT СТСАСTTGAAATAC-ATTACCTGA-------АТССTCT-------AGAGGTGGTTCTACT CTCACTCOCT CTCACTGAAATAC-ATTAGCTG-A---ATCCICT---TCACTTGAAATAC-ATTAGCTGA-------ATCC CTCACTTGAAATAC-ATTAGCTGA-------ATCCTCT-------AGAGGTGGTTCTACT CTCACTTGAAATAC-ATTAGCTGA------ATCCTCT------AGAGGTGGTTCTACI CTCACTTGAAATAC-ATTAGCTGA-------ATCCTCT-------AGAGGTGGTTCTACT CTCGCCTTAAAAGAG-TTAGTGGT----AATAACATC---------------CACGGCTA CTCGCCTTAAAAGA-GTTAGTGGT----AATAACATC--------------CACGGCTA CTCGCCTTAAATGAC-TTAGTGGG-----AATAGCATC---------------CACGGCT CTCGCCTTAAATGA-CTTAGTGGG----AATAGCATC---------------CACGGCTA СТCGCСTTAAAAGA-GTTAGTGGT--CTCGCCTTAAARGA-GTTAGTGGT--CTCGCT TAAAGA-GT

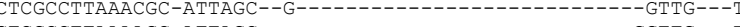
GGTTG---T CTCGCCTIAAAAGC-ATTAGC--G--------

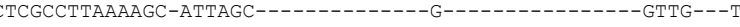
CTCGCCTTAAAAGC-ATTAGC-------------G--------------GTTG---T CTCGCCTTAAAAGC-ATTAGC-------------G--------------GTTG---T CTCGCCTTAAAAGC-ATTAGC--------------G--------------GTTG---T CTCATCTTAAAAGT-ATTAGCTGG-------ATCTGTC---TATATGACTGGTTTGACT CTCATCTTAAAAGT-ATTAGCTTG-------GATCTGTC---TATATGACTGGTTTGACT CTCATCTTAAAAGT-ATTAGCTGG-------ATCTGTC---TATATGACTGGTTTGACT CTCGTCTTAARAGT-ATTHCCTGG---0--ATCTGTC--TATATCACTGGTTTGACT 作 GGTTTGAC' TC---TATATGACTGGTTTGACT CTCATCTTAAAAGT-ATTAGCTA----_GATCTGTC---TCTATAACTGGTTTGAC' TCTATGACTGGTTTGAC CTCATCTTAAAAGT-ATTAGCTAG--------ATCTGTC---TCTATGACTGGTTTGACT CTCATCTTAAAAGT-ATTAGCTAG--------ATCTGTC---TCTATGACTGGTTTGACT CTCATCTTAAAAGT-ATTAGCTAG--------ATCTGTC---TCTATGACTGGTTTGACT CTCATCTTAAAAGT-ATTAGCTAG--------ATCTGTC---TCTATGACTGGTTTGACT

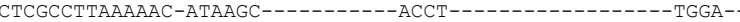

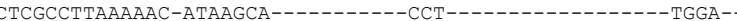

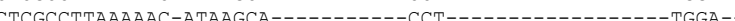
CTCGCCTTAAAARC-DTAGCA-CTCGCCITAAAAAC-ATAAGCA---T-UGACTCGCCITAAAAAC-ATAACC---0-0--0CTCGCCTTAAAAAC-ATAAGC--A-------CCT-----CTCGCCTTAAAAAC-ATAAGC----------ACCT--------------TGGACTCGCCTTAAAAAC-ATAAGC-----------ACCT-----------------TGGA-CTCGCCTTAAAGAT-ATAAGC--A--------CCT---------------TGGA-

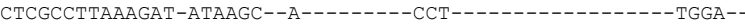

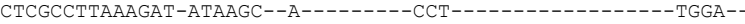
CTCGCCTTAAAGAC-ATAAGC---A--------CCT--T--------------GGA--

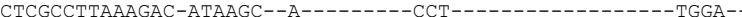

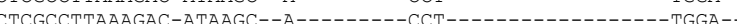
CTCGCCTTAARGACATAGC--A CTCGCCIAAAGAC-AIAAGC-ACTCGCCITAAAGAC-ATARGC-ATCGCCITAAAGAC-ATAAGC---A-2----GGA-

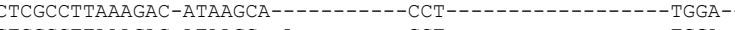

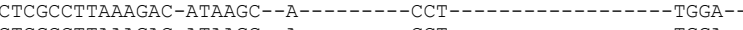
CTCGCCTTAAAGAC-ATAAGC--A--------CCT--------------TGGACTCGCCTTAAAGAC-ATAAGC----------ACCT----------------TGGACTCGCCTTAAAGAC-ATAAGC---A--------CCT--T---------------GGA- 
R. minuta AF190010 R. Slooffiae AF444552 R. slooffiae AF444627 Cy. macerans AF444317

Cy. macerans AF444329 Uncultured fungus AB520409 Uncultured fungus AB520402 Uncultured fungus AB520396 c. sp. $A F 444448$

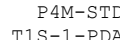

C. macerans EU082232

C. macerans EU082233

C. macerans EU082231

C. macerans AY052490 Uncultured fungus EF504408

Bm. albus AF444664 $\mathrm{V} 5 \mathrm{M}-\mathrm{PDA}$

B. unica AF444441

Bm. albus AB093527

$\mathrm{Bm}$. albus CBS7856

C. dimennae AY188365

C. dimennae AF410473

C. dimennae EU266559 Uncultured Fungus AM901805

$$
\begin{array}{r}
\text { T1S-3-PDA } \\
\text { Y5S-PDA }
\end{array}
$$

TS-1-STD

C. oeirensis AY188362

C. magnus AF190009

Y $2 \mathrm{M}-\mathrm{PDA}$

C. oeirensis $\mathrm{AF} 444349$

C. oeirensis AF444364

C. oeirensis EU002866

C. chernovii AF444354

C. chernovii FN400936

Uncultured fungus FM178256 U3S-PDA

Uncultured fungus AJ875389 Uncultured fungus AB 20331

40 -1
-1 Z2S-STD

C. carnescens EU149785 C. sp. HM849618

C. carnescens CBS8395

C. carnescens AB105438

C. victoriae AY188380

C. victoriae AJ581046

C. victoriae AM160647

C. victoriae CBS9265

C. victoriae HM84962

c. victoriae Hus 9622

C. victoriae

C. Victoriae AY04065 L2S-1-PDA L2S-1-PDA
W5S-4-STD

C. sp. HM589279

C. victoriae AY040654 $\mathrm{S} 4 \mathrm{~S}-\mathrm{PDA}$

C. victoriae CBS9267
TGGCGTAATAAT-TATTTCG---------C------------------TAAGGA-CGT

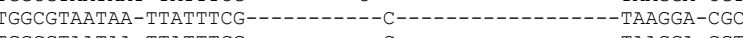

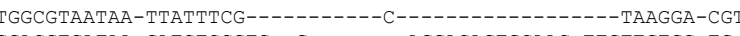
CGACGTGATAA-GATCTCCGTC--G-------AGGACAGTGCAAC-TTGTTGTGC-TGCGACGTGATAA-GATCTCCGTC--G-------AGGACAGTGCAAC-TTGTTGTGC-TGCGACGTGATAAG-ATCTCCGTC---------GAGGACAGTGCAACTTGTT--GTGCTGCGACGTGATAAG-ATCTCCGTC----------GAGGACAGTGCAACTTGTT--GTGCTGCGACGTGATAAG-ATCTCCGTC---_-----_GAGGACAGTGCAACTTGTT--GTGCTGCGACGTGATAA-GATCTCCGTC-G----_----AGGACAGTGCAACTT-GTTGTGC-TG CGACGGATAB CGACGTGATAAG-ATCTCCGIC--G--0--AGGACAGIGCAAC-TTGTTGTGC-TG CGACGTGTAA-GATCTCCGTC---0-0---GAGGACAGTGCAAC-TIGTTGGC-TG CGACGTGATA-GATCTCCGIC-G-CGACGTGATAA-GATCTCCGTC-G--------AGGACAGTGCAACTT-GTTGTGC-TGCGACGTGATAA-GATCTCCGTC-G--------AGGACAGTGCAACTT-GTTGTGC-TG CGACGTGATAA-GATCTCCGTC-G--------AGGACAGTGCAACTT-GTTGTGC-TGAGACGTAATAAG---TTTCGTC-----------T------------GGTCAAGGTG-TGA

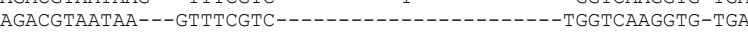
AGACGTAATAAG---TTTCGTC-----------T------------GGTCAAGGTG-TGA

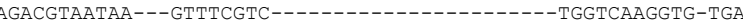

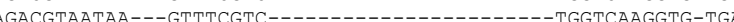
TACTHATAA 作

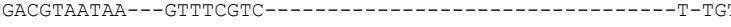
TGACGTAATAA---GTTTCGTC--------0--10

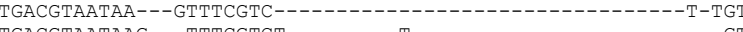

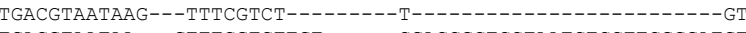
TGACGTAATAA---GTTTCGTCTTGT------CGACCGCTCCTAATCTCCTTCGGGATGT TGACGTAATAAG---TTTCGTCT---------T----------------------GT

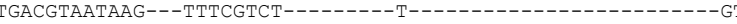
TGGCATAATAAGTATTTTGCTG-A------------G----GACAT-CTTCGGA-TGTGGCATAATAAGTATTTTGCTG-A--------------G-----GACAT-CTTCGGA-TG-

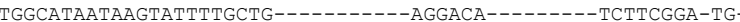

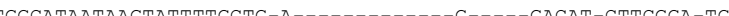
TGGCATAATAAGTITGCTG-A---0-TGGCATARTAATATTCTCGA-TG GGCATAATAAGIATIITGCTGAG--1-0-1TCGG-TG IGGCATAATAAGTATTTTGCTG-------A--------GGACAT-CTTCGGA-TG

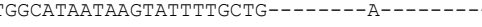
TGGCATAATAAGTATTTTGCTG---------------AGGACATCTTCGGA-TGTGGCATAATAAGTATTTTGCTG----------AGGACA--------TCTTCGGA-TGTGGCATAATAAGTATTTTGCTG----------AGGACA--------TCTTCGGA-TG

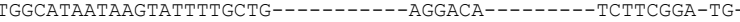

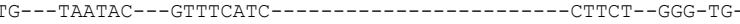
TG---TAATACG---TTTCATC----------C-------------TTCT---GGGTG-

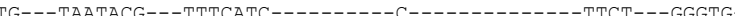
TG- TATCG

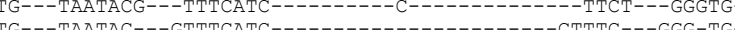
TG--TAATAC-OTITCATC-C-TGTG---TAATAC---GTTTCATC-C-------------TTCT--GGG-TG TG---TAATAC---GTTTCATC-----------------CTTCT--GGG-TG TG---TAATAC---GTTTCATC-------------------CTTCT--GGG-TG TG---TAATAC---GTTTCATC-C-----------------TTCT--GGG-TG TG---TAATAC---GTTTCATC-C----_-_-

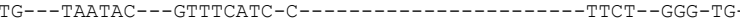
TG---TAATAC---GTTTCATC--C-----------------TTCT--GGG-TG TG---TAATAC---GTTTCATC-C--_-_-_-_-_-_-_-_-----TTCT--GGG-TG TG---TAATAC---GTTTCATC-C-_-_-_-_-_-_-_-_-_-_TTCT--GGG-TG TG IG-DAATAC--GTICATC-C--

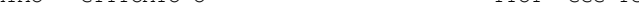

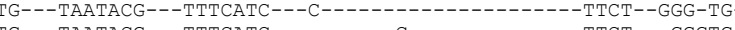
TG---TAATACG---TTTCATC---------C----------TTCT---GGGTG TG---TAATAC---GTTTCATC-C----------------TTCT--GGG-TG TG---TAATAC---GTTTCATC-C--------------TTCT--GGG-TG TG---TAATAC---GTTTCATC---------C-------------TTCT--GGG-TGTG---TAATAC---GTTTCATC--C-----------------TTCT--GGG-TG 
R. minuta AF190010 R. Slooffiae AF444552 R. slooffiae AF444627 Cy. macerans AF444317

Cy. macerans AF444329 Uncultured fungus AB520409 Uncultured fungus AB520402 Uncultured fungus AB520396 C. sp. AF444448

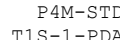

C. macerans EU082232

C. macerans EU082233

C. macerans EU082231

C. macerans AY052490 Uncultured fungus EF504408

Bm. albus AF444664 $\mathrm{V} 5 \mathrm{M}-\mathrm{PDA}$

B. unica AF444441

Bm. albus AB093527

$\mathrm{Bm}$. albus CBS7856

C. dimennae AY188365

c. dimennae AF410473

C. dimennae EU266559 Uncultured Fungus AM901805 $\mathrm{T} 1 \mathrm{~S}-3-\mathrm{PDA}$
$\mathrm{Y} 5 \mathrm{~S}-\mathrm{PDA}$ Y $5 \mathrm{~S}-\mathrm{PDA}$

C. oeirensis AY188362

C. magnus AF190009 Y $2 \mathrm{M}-\mathrm{PDA}$

C. oeirensis $A F 444349$

C. oeirensis AF444364

C. oeirensis EU002866

C. chernovii AF444354

C. chernovii FN400936 Uncultured fungus FM178256 U3S-PDA

Uncultured fungus AJ875389 Uncultured fungus AB520331
C. carnescens AB035050

ltured fungus Uncultured fungus BJ8?

Uncultured fungus AM901777 Z2S-STD

C. Carnescens EU149785 C. sp. HM849618 C. carnescens CBS8395 C. carnescens AB105438 C. victoriae AY188380 C. victoriae AJ581046 C. victoriae AM160647 C. victoriae CBS9265 C. victoriae HM84962 C. victoriae $A 5444645$ C. victoriae Ap 444645

c. victoriae AY04065 L2S-1-PDA $22 S-1-P D A$
$W 5 S-4-S T D$ C. sp. HM589279 c. victoriae AY040654
S4S-PDA

C. victoriae CBS9267
-

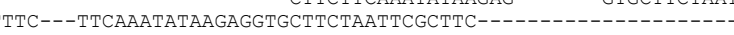
TTC---TTCAAATATAAGAGGTGCTTCTAATTCGCTTC--

-GCCGCTCCTAGCAGTTGACGTCGCTTCTAATTAGCGCAGACTTCGAG--TGC-----TG -GCCGCCGCTAGCAGTTGAT-ACGCTTCTAATTAGCGCAGACTTCGAG--TGC-----TG -GCCGCCGCTAGCAGTTGA-TACGCTTCTAATTAGCGCAGACTTCGA--GTGC-----TG -GCCGCCGCTAGCAGTTGA-TACGCTTCTAATTAGCGCAGACTTCGA--GTGC-----TG -GCCGCCGCTAGCAGTTGA-TACGCTTCTAATTAGCGCAGACTTCGA--GTGC-----TG

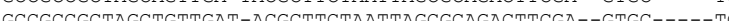
- GCCCCCGCTAGCaGTTGAT-HCGCTTCTARTTAGCGCAGaCTTCGA--GTGC----TG -GCCGCTCCTACCAGTGACACTC -GCCGCTCCIAGCAGTIGACGCGCTICTAATIACCGCAGACICGA-GTGC--TG -GCCGCCGCTAGCAGTTGAT-ACGCTTCTAATTAGCGCAGACTTCGA--GTGC-----TG -GCCGCCGCTAGCTGTTGAT-ACGCTTCTAATTAGCGCAGACTTCGA--GTGC-----TG -GCCGCCGCTAGCTGTTGAT-ACGCTTCTAATTAGCGCAGACTTCGA--GTGC-----TG CGAC------------T-------GCT---CATAACCC--GACCGCAA------------G CGAC-----------T-------GCT---CATAA--CCCGACCGCAA------------G AGCC-----------T-------GCT---CATAACCC--GACCGCAA------------G AGCC-----------T-------GCT---CATAA--CCCGACCGCAA------------G CGAC-----------T-------GCT---CATAA--CCCGACCGCAA------------G CGAC-----------T-------GCT---CATAA--CCCGACCGCAA-----------CGAC---_-_-_-_-_----_CGCT---CCTAATCTCCTTCGGGA----TGTCTCT--G CGAC----------C-----GCT---CCTAATCTCCTTCGGGA----TGTCTCTAGG CGAC--CGAC---------C------GCT---CCTAATCTCCTTCGGGA----TGTCTCTAGG TCTAGGGACACTTCATGAT-

CGAC--------C------GCT---CCTAATCTCCTTCGGGA----TGTCTCTAGG CGAC-----------C-------GCT---CCTAATCTCCTTCGGGA----TGTCTTTAGG -GCCAGGACCTAGACTATTGTCTGCT--AACTAAACCATCACTTTAA--GTGCATCCTTG -GCCAGGACCTAGACTACTGTCTGCT--AACTAAACCATCACTTTAA--GTGCATCTTTC -GCCAGGACCTAGACTATTGTCTGCT--AACTAAACCATCACTTTAA--GTGCATCTTTG -GCCAGCACCTAACTATA 作 -GCCAGGACCTAGACTATTGTCIGCT--AACTAAACCATCACTTTAA--GTGCATCTTTG -GCCAGGACCTAGACTATTGTCTGCT--AACTAAACCATCACTTTAA--GTGCATCTTTG -GCCAGGACCTTGACTATGTOTCT--AACTAAACCATTACTTTAA--GTGCATCTTT -GCCAGGACCTAGACTATTGTCTGCT--AACTAAACCATCACTTTAA--GTGCATCTTCG -GCCAGGACCTAGACTATTGTCTGCT--AACTAAACCATCACTTTAA--GTGCATCTTTG -GCCAGGACCTAGACTATTGTCTGCT--AACTAAACCATCACTTTAA--GTGCATCTTCG -GCCAGGACCTAGACTATTGTCTGCT--AACTAAACCATCACTTTAA--GTGCATCTTCG

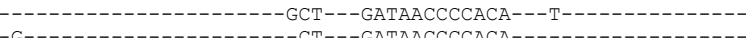
-

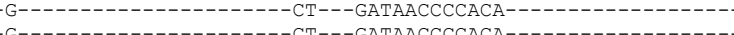
G--

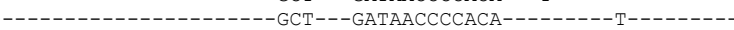

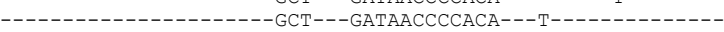

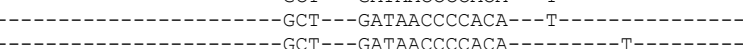

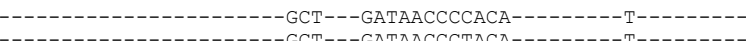

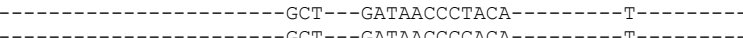
---------------GCT---GATAACCCCACA--------T-------------------------GCT---GATAACCCCACA---------T--------

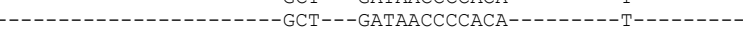

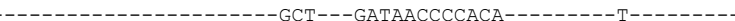
-

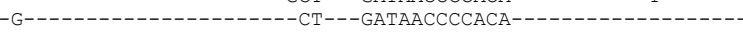
----------------------GCT---GATAACCCCACA---------T--------

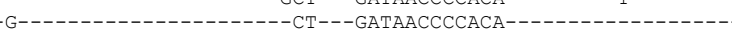

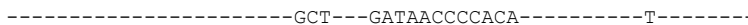


R. minuta AF190010 R. Slooffiae AF444552 R. slooffiae AF444627 Cy. macerans AF444317 Cy. macerans AF444329 Uncultured fungus AB520409 Uncultured fungus AB520402 Uncultured fungus AB520396

C. sp. $A F 444448$ P4M-STD
T1S-1-PDA

C. macerans EU082232

C. macerans EU082233

C. macerans EU082231

C. macerans AY052490 Uncultured fungus EF504408

Bm. albus AF444664 $\mathrm{V} 5 \mathrm{M}-\mathrm{PDA}$

B. unica AF444441

Bm. albus AB093527

Bm. albus CBS7856

C. dimennae AY188365

C. dimennae AF410473

C. dimennae EU266559 Uncultured Fungus AM901805 $\mathrm{T} 1 \mathrm{~S}-3-\mathrm{PDA}$
$\mathrm{Y} 5 \mathrm{~S}-\mathrm{PDA}$ Y $5 \mathrm{~S}-\mathrm{PDA}$

C. oeirensis AY188362

C. magnus AF190009 Y $2 \mathrm{M}-\mathrm{PDA}$

C. oeirensis $\operatorname{AF} 444349$

C. oeirensis AF444364

C. oeirensis EU002866

C. chernovii AF444354

C. Chernovii AF444354 Uncultured fungus FM178256 U3S-PDA

Uncultured fungus AJ875389 Uncultured fungus AB520331 820684

Uncultured fungus AM901777 Z2S-STD

C. Carnescens EU149785 C. sp. HM849618 C. carnescens CBS8395 C. carnescens AB105438 C. victoriae AY188380 C. victoriae AJ581046 C. victoriae AM160647 C. victoriae CBS9265 C. victoriae HM84962 C. victoriae Hu849622 c. victoriae AF 444645

c. victoriae AY04065 L2S-1-PDA $2 \mathrm{~S}-1-\mathrm{PDA}$
$\mathrm{W} 5 \mathrm{~S}-4-\mathrm{STD}$

C. sp. HM589279

C. victoriae AY04065 S4S-PDA

C. victoriae CBS9267
GCGCTTTATAGCAC--.--_-_tTTAAGCTTTAGATCTCAAATCAGTCAGGACTACCCG ----TAATAGCAT---------TTAAGCTTTAGACCTCAAATCAGTCAGGACTACCCG ----TAATAGCAT--------TTAAGCTTTAGACCTCAAATCAGTCAGGACTACCC GCAACTTTTGACA------A----------CTTGGC-CTCAAATCAGGTAGGACTACCCA GCAACTTTTGACA-------A----------CTTGGC-CTCAAATCAGGTAGGACTACCCA GCAACTTTTGACA------------ACTTGGC-CTCAAATCAGGTAGGACTACCCA GCAACTTTTGACA--------------ACTTGGC-CTCAAATCAGGTAGGACTACCCA GCAACTTTTGACA--_-_-_-_-----ACTTGGC-CTCAAATCAGGTAGGACTACCCA

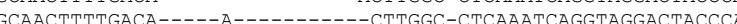
CCACT GCAАCTTTGACA-

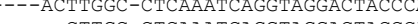

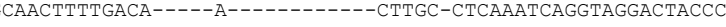
GCAACTTTTGACA-----A--------_CTTGGC-CTCAAATCAGGTAGGACTACCCA GCAACTTTTGACA-----A--------CTTGGC-CTCAAATCAGGTAGGACTACCCGCAACTTTTGACA-----A--------CTTGGCTCTCAAATCAGGTAGGACTACCCA GTCATTATT--TAT-------------GCTCTGAC-CTCAAATCAGGTAGGACTACCCG GTCATTATT--TAT-------------GCTCTGAC-CTCAAATCAGGTAGGACTACCCG GTCACTATT--TAT------------_GCTCTGAC-CTCAAATCAGGTAGGACTACCCG GTCACTATT--TAT-------------GCTCTGAC-CTCAAATCAGGTAGGACTACCCG GTCATTATTT-TAT--------------GCTCTGAC-CTCAAATCAGGTAGGACTACCCG

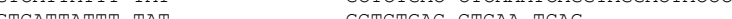
G CACAT-

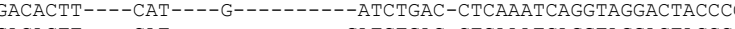
GACACIT-O-CATGACACTT----CAT----G-------ATCTGAC-CTCAAATCAGGTAGGACTACCCG

GACACTT----CAT-----------GATCTGAC-CTCAAATCAGGTAGGACTACCCG GACACTT----CAT-------------GATCTGAC-CTCAAATCAGGTAGGACTACCCG GATGTTACT--CATTGTGTAACTTTG-ACATCTGGC-CTCAAATCAGGTAGGACTACCCG GATGTTACT--CATTGTGTAACTTTG-ACATCTGGC-CTCAAATCAAGTAGGACTACCCG GATGTCACT--AATCGTGTAACTTTGA-CATCTGGC-CTCAAATCAGGTAGGACTACCCG GATGTACT- AATCTGTAACTTTG-ACATCTGCC-CTCAAATCAGGTAGGACTACCCG GACTACCC

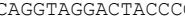
GATGTTACT--AATTGTGTAACTTTG-ACATCTGGC-CTCAAATCAGGTAGGACTACCCG GATGTTACT--CATTGTGTAACTTTGA-CATCTGGC-CTCAAATCAGGTAGGACTACCC GATGTTACT--CATTGTATAACTTTGA-CATCTGCC-CTCAATCACGTACGACTACCC GATGTTACT--CATTGTGTAACTTTGCACATCTGGC-CTCAAATCAGGTAGGACTACCCG GATGTTACT--CATTGTGTAACTTTGA-CATCTGGC-CTCAAATCAGGTAGGACTACCCG GATGTTACT--CATTGTGTAACTTTGA-CATCTGGC-CTCAAATCAGGTAGGACTACCCG GATGTTACT--CATTGTGTAACTTTGA-CATCTGGC-CTCAAATCAGGTAGGACTACCCG

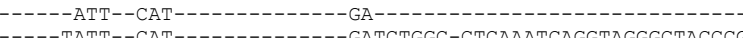
----TATT--CAT----_-----GATCTGGC-CTCAAATCAGGTAGGGCTACCCG ----TATT--CAT------------GATCTGGC-CTCAAATCAGGTAGGGCTACCCG ---ATT--CAT----G------ATCTGGC-CTCAAATCAGGTAGGGCTACCC ------ATT--CAT-------------GATCTGGC-CTCAAATCAGGTAGGGCTACCCG ----ATT--CAT----G-------ATCTGGC-CTCAAATCAGGTAGGGCTACCCG ----ATT--CAT----G--------ATCTGGC-CTCAAATCAGGTAGGGCTACCCG -----ATT--CAT-----G--------ATCTGGC-CTCAAATCAGGTAGGGCTACCCG -----ATT--CAT----G---------ATCTGGC-CTCAAATCAGGTAGGGCTACCCG

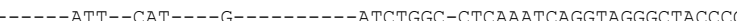
-1AAT-CAT--0 ---ATT--CAT----G-------AACTGGC-CTCAAATCAGGTAGGGCTACCCG ----ATT--CAT----G-------ATCTGGC-CTCAAATCAGGTAGGGCTACCCG ----TATT--CAT------------GATCTGGC-CTCAAATCAGGTAGGGCTACCCG -----ATT--CAT-----G-------ATCTGGC-CTCAAATCAGGTAGGGCTACCCG 
R. minuta AF190010

R. Slooffiae AF444552

R. slooffiae AF444627

Cy. macerans AF444317

Cy. macerans AF444329 Uncultured fungus AB520409 Uncultured fungus AB520402 Uncultured fungus AB520396

C. sp. AF444448 P4M-STD

C. macerans EU082232

C. macerans EU082233

C. macerans EU082231

C. macerans AY052490

Uncultured fungus EF504408

Bm. albus AF444664 $\mathrm{V} 5 \mathrm{M}-\mathrm{PDA}$

B. unica AF444441

Bm. albus AB093527

$\mathrm{Bm}$. albus CBS7856

C. dimennae AY188365

C. dimennae AF410473

C. dimennae EU266559

Uncultured Fungus AM901805 T1S-3-PDA $\mathrm{Y} 5 \mathrm{~S}-\mathrm{PDA}$

C.

C. magnus AF190009 Y $2 \mathrm{M}-\mathrm{PDA}$

C. oeirensis AF444349

C. oeirensis AF444364

C. oeirensis AF444364

C. oeirensis EU002866

C. Chernovii AF444354

Uncultured fungus FM178256 U3S-PDA

Uncultured fungus AJ875389

Uncultured fungus AB520331

AB035050

Uncultured fungus FJ820684

Uncultured fungus AM901777

C. Carnescens EU149785

C. sp. HM849618

C. carnescens CBS8395

C. carnescens AB105438

C. victoriae AY188380

C. victoriae AJ581046

C. victoriae AM160647

C. victoriae CBS9265

C. victoriae HM849622

C. victoriae AF444645

C. victoriae AY040656

C. victoriae AY040656 L2S-1-PDA TSS-4-STD

C. sp. HM589279

C. victoriae AY040654

C. victoriae CBS9267
CTGAACTTAAGCATATCAATAAGCGGAGGAAAAG-...-...-...

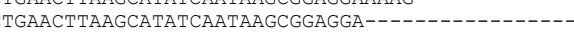
CTGAACTTAAGCATATCAATAAGCGGAGGAAAAG-----------CTGAACTTAAGCATATCAATAAGTGGAGGA-

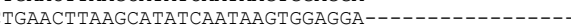
CTGAACTTAAGCATATCAATAAGTGGAGGAAAAGAAACTAACAAGGA CTGAACTTAAGCATATCAATAAGTGGAGGAAAAGAAACTAACAAGGA CTGAACTTAAGCATATCAATAAGTGGAGGAAAAGAAACTAACAAGGA

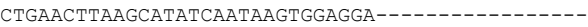

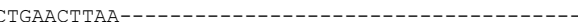

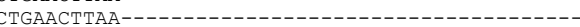

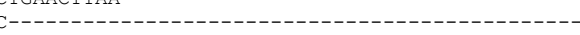

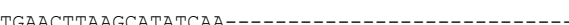

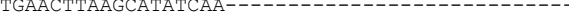

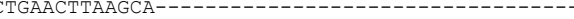
CTGAACTTAAGCATATCAATAAGCGGAGGA--.--CTGAACTTAACCA

CTGAACTTAAGCATATCAATAAGCGGAGGA-................. CTGAACTTAAGCATATCAA

CTGAACTTAAGCaTATCARTARGCGGaGGaAaAGaAaCTaACaAGGa CTCAACITAAGCATATCAATARGCGGAGGA--O--U CTGAACTTAAGCATATCAATAAGCGGAAAAGAAAATTATTGAAATT -

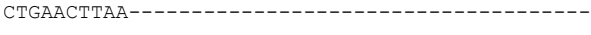
CTGAACTTAA-CTGAACTTAA--

CTGAACTTAAGCATATCAATAAGCGGAGGAAAAGAAACTAACAAGGA

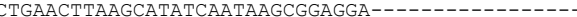
CTGAACTT每 TTAACTTAAGCATATCAATAAGCGGAGGA-TGAACTTAA

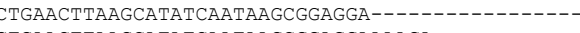
CTGAACTTAAGCATATCAATAAGCGGAGGAAAAGA----------CTGAACTTAAGCATATCAATAAGCGGAGGA-

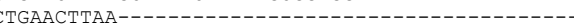

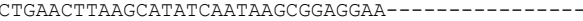
CTGAACTTAAGCATATCAATAAGCGGAGGAAAAGAAACTAACAAGGA

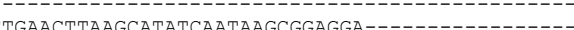

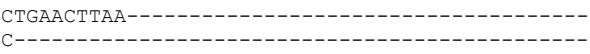

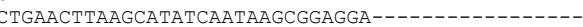

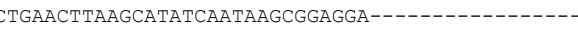

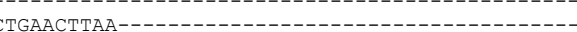
CTGAACTTAAGCATATCAATAAGCGGAGGAAAAGAAACTAACTAGGA

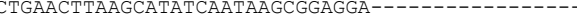
CTGAACTTAAGCATATCAATAAGCGGAGGAAAAGAAACTAACTAGGA CTGAACTTAAGCATATCAATAAGCGGAGGAAAAGAAACTAACTAGGA CTGAACTTAAGCATATCAATAAGCGGAGGA-

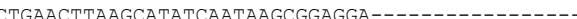
CTCABCTTAHGCATATCARTAAGCGGAGGA-0

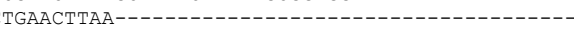

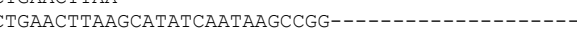
CTGAACTTAAGCATATCAATAAGCGGAGGA-

-

CTGAACTTAAGCATATCAATAAGCGGAGGAAAAGAAACTAACTAGGA 


\subsubsection{Pucciniomycotina}

C. chernovii FN400936 C. magnus AF190009 C. oeirensis AF444364 E5S-PDA Fungal sp. GU213437 R. mucilaginosa AF444614 R. mucilaginosa AF444609 R. mucilaginosa R. mucilaginosa AF444503 R. mucilaginosa CBS2382 R. mucilaginosa FR690147
R. mucilaginosa HQ702343 R. mucilaginosa HQ702343 R. mucilaginosa FJ515212
R. mucilaginosa EU149812 R. mucilaginosa EU871492 Uncultured fungus FM875856

R. mucilaginosa CBS8618 R. mucilaginosa AF444635

K. malvinella AF444498

K. malvinella DQ667165

K. aeria AM922224

K. aeria AF444562

K. aeria AF444675 H1OS-PDA

Basidiomycete BSU65613

R. sp. FJ238090 P2S-PDA

R. oryzicola AY335160 $R$.

S. dacryoidea AF444571 Z4S-1-PDA

S. dacryoidea AF444597

S. dacryoidea AF444500

S. dacryoidea AB049027

. dacryoidea DQ832207

R. minuta AB078490

R. minuta $A B 07848$

R. minuta $A B 038117$

R. minuta AB078501

R. minuta AF190010

R. minuta AB078493

R. minuta AB078493
R. minuta AB078497 B078497
I1-STD

R. sp. AF 444619 R. Slooffiae FJ515215 R. Slooffiae AB025994
J3S-STD

R. Slooffiae FJ807685 R. slooffiae AF444589 R. Slooffiae AB078491 R. slooffiae $A B 052212$ R. Slooffae AB052212 R. Sloofflae AF444627 R. Slooffiae AF444552

R. Slooffiae AB078495
R. slooffiae CBS8411

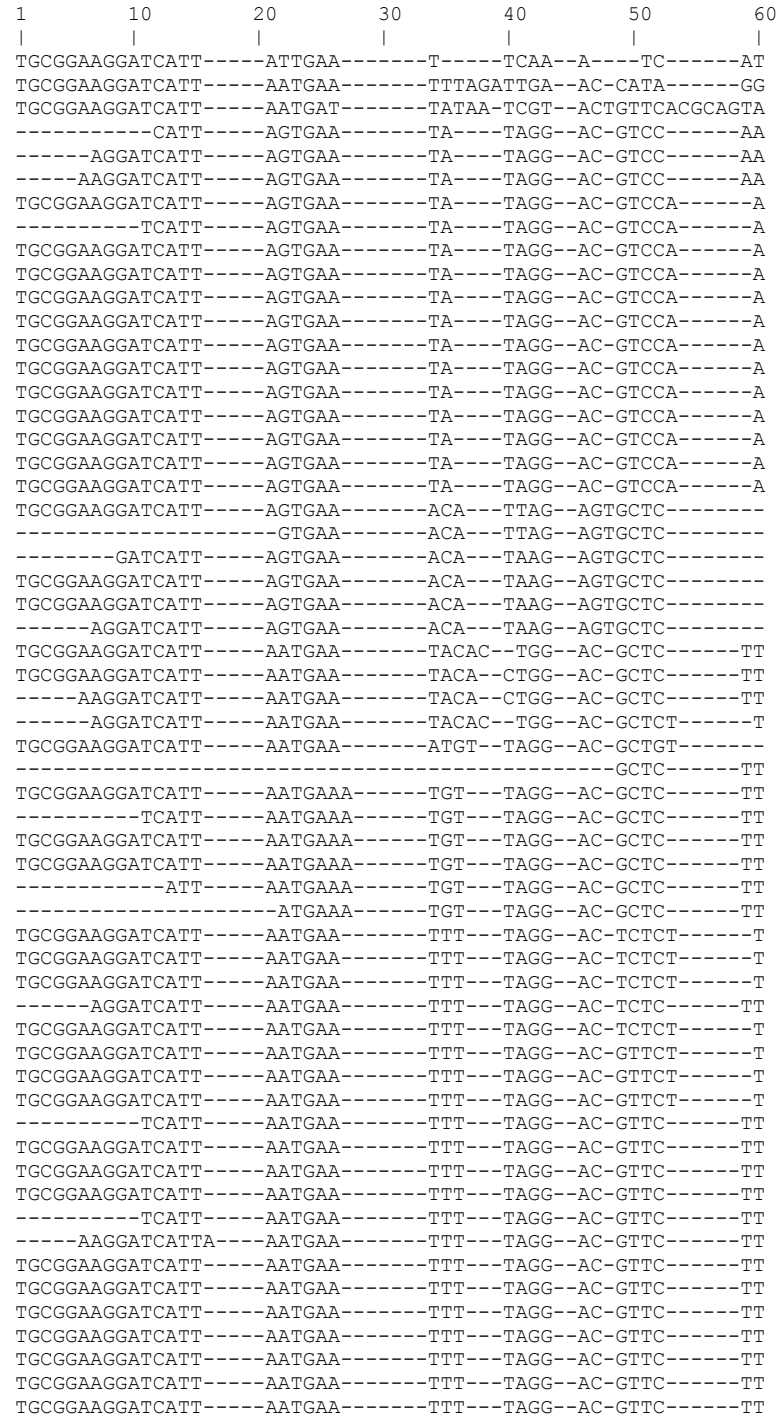


C. chernovii FN400936 C. magnus AF190009

C. oeirensis AF444364 E5S-PDA

Fungal sp. GU213437

R. mucilaginosa AF444614 Q5S-2-PDA

R. mucilaginosa AF444609

R. mucilaginosa AF444503

R. mucilaginosa CBS2382

R. mucilaginosa FR690147

R. mucilaginosa HQ702343
R. mucilaginosa FJ515212

R. mucilaginosa EU149812

R. mucilaginosa EU871492 Uncultured fungus FM875856

R. mucilaginosa CBS8618 R. mucilaginosa AF444635 K. malvinella AF444498 K. malvinella AF444498 K. aeria AM922224 K. aeria AM922224
K. aeria AF444562 K. aeria AF444562
K. aeria AF444675 H10S-PDA

Basidiomycete BSU65613 R. sp. FJ238090

R. sp. FJ865356 P2S-PDA

R. oryzicola AY335160 R. sp. FJ008055

S. dacryoidea AF444571 24S-1-PDA

S. dacryoidea AF444597

S. dacryoidea AF444500

S. dacryoidea AB049027

. dacryoidea DQ832207

. minuta AB078490

R. minuta $A B 078484$

R. minuta $A B 038117$ B 4M-STD

R. minuta $A B 078501$

R. minuta AF190010

R. minuta AF 190010

R. minuta AB078497 I1-STD

R. sp. AF444619

R. Slooffiae FJ515215

R. slooffiae AB025994 J $3 S-S T D$

R. Slooffiae FJ807685

R. Slooffiae AF 444589

R. slooffiae AB078491

R. slooffiae AB052212

R. Slooffiae AF444627

R. slooffiae AF444552

R. slooffiae AB078495

R. slooffiae CBS8411
CT--GTGGCT--TCG-GT------CCGGATGTGCCA--ACAGG-------TA--ACACTG CG--AAAGCC--AGTGGT------TCTTCT-----T--TCAT---ATCCATA--ACACC IG--TGGGGTGGTGACTT------CGGTCC---CGC--TCATTCATATCCATA-ACACCT CT--TAACT---TGGAGT------CCGAAC----TC--TCACT--TT--CTA--ACCCTCT--TAACT---TGGAGT------CCGAAC----TC--TCACT--TT--CTA--ACCCTCT--TAACT---TGGAGT------CCGAAC----TC--TCACT--TT--CTA--ACCCTCT--TAACT---TGGAGT------CCGAACT----C--TCACT--TT--CTA--АCCCTCT--TAACT---TGGAGT------CCGAACT----C--TCACT--TT--CTA--ACCCTСT--TAACT---TGGAGT-----CCGAACT----C--TCACT--TT--CTA--ACCCTCTCT

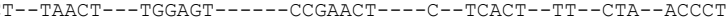
CT--TAACT---TGGAGT-----CCGAACT----C--TCACT--TT--CTA--АCCCTCT--TAACT---TGGAGT-----CCGAACT----C--TCACT--TT--CTA--ACCCTCT--TAACT---TGGAGT------CCGAACT----C--TCACT--TT--CTA--ACCCT CT--TAACT---TGGAGT------CCGAACT----C--TCACT--TT--CTA--ACCCTCT--TAACTT---GGAGT------CCGAACT------CTCACT--TT--CTA--ACCCTCT--TAACT---TGGAGT------CCGAACT----C--TCACT--TT--CTA--ACCCTCT--TAACT---TGGAGT------CCGAACT----C--TCACT--TT--CTA--ACCCTCTCAT-T-----GAGGC--0--TCG--0---TT--TAACT--ATCCATTACACTTT-

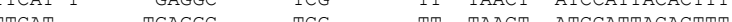

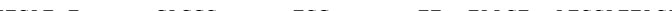
TCAT-T--

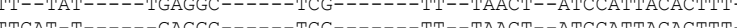

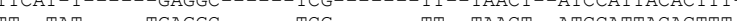
TT--TAT-----TGAGGC------TCG------TT--TAACT--ATCCATTACACTTTTT--TA------GAGGT-----CCGACC----CT--TCA---TTACCTT-CACACT TT--TA-------GAGGT------CCGACCC------TTCAT----TACCTT-CACACTTT--TA------GAGGT-----CCAACCC-----TTCAT---TACCTT-CACACT TT--TA-------GAGGT------CCGACCC----T--TCA----TTACCTTC-ACACTTT--TA------CAGGT------CCGACC---CTT--TTA----TTACCTTTCACACTTT--TA-------GAGGT------CCGACCA-----ACTCATT--TTACCTTTCACACT

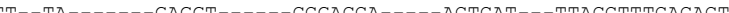
TI TT- TATI-TA-DTTT--TA-----GAGGT-----CCGACCA-----ACTCATT--TTACCTTTCACACTTT--TA--TT--TA-------GAGGT------CCGACCA-----ACTCATT--TTACCTTTCACACTTT--TA------GAGGT-----CCGACCA---TT--TCA----TTTCCTT--ACACTTT--TA-------GAGGT------CCGACCA---TT--TCA----TTTCCTT--ACACT TT--TA------GAGGT-----CCGACCA---CT--TCA----TTTCCTT--ACACTTT--TA------GAGGT------CCGACC---ACT--TCA----TTTCCTT--ACACTTT--TA-------GAGGT------_CCGACCA---CT--TCA----TTTCСТT--ACACT TT--TA--0-GAAT----CCGACCA--TT--TCATT--TT--CTT--ACACTTTTT- TA- TT-TAIT--TA--1-GAAGT----CCGACC---CTT--TCATT--TT--CTT--ACACCTT--TA---_-GAAGT-----CCGACC----CITICATI--TI--CIT--ACACCTT--TA-------GAAGT------CCGACC-----CTTTCATT--TT--CTT--ACACCTT--TA------GAAGT-----CCGACC----CCTTCATT--TT--CTT--ACACCTT--TA-------GAAGT------CCGACC---CTT--TCATT--TT--CTT--ACACTTT--TA------GAAGT-----CCGACC----CTTTCATT--TT--CTT--ACACTTT--TA------GAAGT-----_CCGACC-----CTTTCATT--TT--CTT--ACACTTT--TA-------GAAGT------CCGACC-----CTTTCATT--TT--CTT--ACACT TT--TA---0-CAACT----CCGACC----CTTTCATT--TT--CTT--ACACTTTTI-TT-TATT--TA-------GAAGT------CCGACC-----CTTTCATT--TT--CTT--ACACT- 
C. chernovii FN400936 C. magnus AF19000 C. oeirensis AF444364 E5S-PDA
E3M-STD

Fungal sp. GU213437

R. mucilaginosa AF444614 Q5S-2-PDA

R. mucilaginosa AF444609 R. mucilaginosa AF444503 R. mucilaginosa CBS2382

R. mucilaginosa FR690147

R. mucilaginosa HQ702343

R. mucilaginosa FJ515212

R. mucilaginosa EU149812

R. mucilaginosa EU871492 Uncultured fungus FM875856

R. mucilaginosa CBS8618 R. mucilaginosa AF444635 K. malvinella AF444498 K. malvinella AF444498

K. aeria AM922224

K. aeria AF444562

K. aeria AF444675 H10S-PDA

Basidiomycete BSU6561

R. sp. FJ238090

R. sp. FJ865356

P2S-PDA

R. oryzicola AY335160

R. sp. FJ008055

S. dacryoidea AF444571 $4 \mathrm{~S}-1-\mathrm{PDA}$

S. dacryoidea AF444597

S. dacryoidea AF444500

S. dacryoidea AB049027

S. dacryoidea DQ832207

R. minuta AB078490

R. minuta AB078484

R. minuta $A B 038117$ B 4M-STD

R. minuta $A B 078501$

R. minuta AF190010

R. minuta AF 190010

R. minuta AB078497 I1-STD

R. sp. AF444619

R. Slooffiae FJ515215

R. Slooffiae AB025994 J3S-STD

R. Slooffiae FJ807685

R. Slooffiae AF444589

R. Slooffiae AB078491

R. slooffiae $A B 052212$

R. Slooffiae AB052212

R. Sloolliae AF44462

R. Slooffiae AF444552

R. Slooffiae AB078495
R. slooffiae CBS8411
TTGGTA--_-_-_--ATTCTAT-CCATAA---CACCTGTG-AACTGTTG-----GATGC

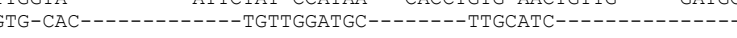

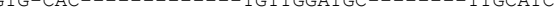

GTG-CAC-T--------TGTTTGGGATAGT--AACTCTCGCAAGAGAGC-----GAACT GTG-CAC-T---------TGTTTGGGATAGT--AACTCTCGCAAGAGAGC-----GAACT GTG-CAC-T--------TGTTTGGGATAGT--AACTCTCGCAAGAGAGC-----GAACT GTG-CAC----------TTGTTTGGGATAGT--AACTCTCGCAAGAGAGC-----GAACT GTG-CAC--------TTGTTTGGGATAGT--AACTCTCGCAAGAGAGC-----GAACT GTG-CAC--0----TTGTTTGGGATAGT--AACTCTCGCAAGAGAGC----GAAC TICACAGAG-----GAAC (1)---GAAC GTG-CAC----------TTGTTTGGGATAGT--AACTCTCGCAAGAGAGC------GAACT GTG-CAC---------TTGTTTGGGATAGT--AACTCTCGCAAGAGAGC-----GAACI GTG-CAC---------TTGTTTGGGATAGT--AACTCTCGCAAGAGAGC-----GAACI GTG-CAC----------TTGTTTGGGATAGT--AACTCTCGCAAGAGAGC-----GAACT GTG-CAC----------TTGTTTGGGATAGT--AACTCTCGCAAGAGAGC-----GAACT GTG-CAT----------TTGTTTGGGATAGT--AACTCTCGCAAGAGGGC-----GAACT GTG-CAT---------TTGTTTGGGATAGT--AACTCTCGCAAGAGGGC-----GAACT GTG-CA-T--0---TTGAAACATT---ACT-TGGTAACCTAG-----GCCT GT-CA-TAG------GGCT (1) 作 GTG-CATTTGAAAACATTACTT--GGATGCTTTGGGCTTCGGTCCTCTG--------TGT GTG-CATTTGA-AACATTACTT--GGATGCTTTGGGCTTCGGTCCTCTG-------TGT GTG-CAC------ACACTTCATTTTTACAC---CCATTTTTAAAC------------AC GTG-CACA------CACTTCATTTTTACAC--CCATTITAAAC------------AC GTG-CACA------CACTTCATTTTTACACGTG-CAC------ACACTTCATTTTTACACC--CATITIT-AAAC---0---AC GTG-CAC-----ATCACTACTTTTTTACACO--CATTTT GTG-CACA----TCACTACTTTTTTACAC-GTG-CCCA-----TCACTACTTTTT-ACAC---CCATTTTTAAAC----------AC GTG-CACA-----TCACTACTTTTTTACAC---CCATTTTTAAAC-----------AC GTG-CACA-----TCACTACTTTTTTACACC--CATTTTTTAAAC------------ACT TG-CACA--0

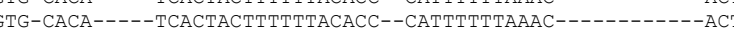
GTG-CACA-----TCACTACTTTTTTACACC--CATTTTTTAAAC------------ACT GTG-CAC------ACACTTCTTTTTCACACA-----TTTT--AAC-----------AC GTG-CAC------ACACTTCTTTTTCACACA-----TTTT--AAC-----------ACT GTG-CAC------ACACTTCTTTT-CACACA-----TTTT--AAC-----------ACA GTG-CAC------ATACTTCTTTT-CACAC----ATTTT---AAC------------ACA GTG-CAC------ATACTTCTTTT-CACACA-----TTTT--AAC------------ACA GTG-CAC-0--ACACTTCTTTTT-ACACA-OCACTTTT-

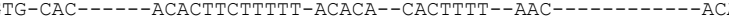
GTG-CAC-----ACACTTCTTTTTTACACA--CACTTTT--AACA----------CC GT-CACA-- CACTICTITITTACACA--CACTTIT--AAC-----------AC GTG-CACA------САСТTCTTTTITACACA--САСТTTT--AAC-----------ACC GTG-CACA------CАСТTCTTTTTTACACA--САСТTTT--AAC-----------ACC GTG-CAC------ACACTTCTTTTTTACACA--CACTTTT--AACA-----------CC GTG-CACA------CACTTCTTTTTTACACA--CACTTTT--AAC-----------ACC GTG-CACA------CАСТTCTTTTTTACACA--САСТTTT--AAC------------ACC

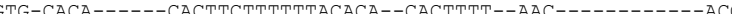
TTG-CACA-GT-CACAG (1) GTG-CACA------CACTTCTTTTTTACACA--CACTTTT--AAC-----------ACC 
C. chernovii FN400936 C. oeirensis AF444364 E5S-PDA
E3M-STD

Fungal sp. GU213437

R. mucilaginosa AF444614 Q5S-2-PDA

R. mucilaginosa AF444609 R. mucilaginosa AF444503 R. mucilaginosa CBS2382

R. mucilaginosa FR690147

R. mucilaginosa HQ702343

R. mucilaginosa FJ515212

R. mucilaginosa EU149812

R. mucilaginosa EU871492 Uncultured fungus FM875856

R. mucilaginosa CBS8618 R. mucilaginosa AF444635 K. malvinella AF444498 K. malvinella DQ667165

K. aeria AM922224 K. aeria AM922224
K. aeria AF444562

K. aeria AF444675 H10S-PDA

Basidiomycete BSU65613

R. sp. FJ238090

R. sp. FJ865356

P2S-PDA

R. oryzicola AY335160

R. sp. FJ008055

S. dacryoidea AF444571 $4 \mathrm{~S}-1-\mathrm{PDA}$

S. dacryoidea AF444597

S. dacryoidea AF444500

S. dacryoidea AB049027

S. dacryoidea DQ832207

R. minuta AB078490

R. minuta $A B 078484$

R. minuta $A B 038117$ B 4M-STD

R. minuta $A B 078501$

R. minuta AF190010

R. minuta AF 190010

R. minuta $A B 078497$ I1-STD

R. sp. AF444619

R. Slooffiae FJ515215

R. Slooffiae AB025994 J3S-STD

R. Slooffiae FJ 807685

R. Slooffiae AF444589

R. slooffiae AB078491

R. Slooffiae AB052212

R. Sloofiae $A F 444627$

R. Slooffiae AF44462

R. Slooffiae AF444552

R. Slooffiae AB078495
R. Slooffiae CBS8411
TTGCATCСАСТ-- TTT----------CACT--TTT------AA--AC-----TAAACATTATTGTAACAAATG----CACT CC-TATTCACT--TAT------AA--AC-----ACAA----AGT-CTATGAATG----TA CC-TATTCACT--TAT------AA--AC-----ACAA----AGT-CTATGAATG----TA CC-TATTCACT--TAT------AA--AC-----ACAA----AGT-CTATGAATG----TA CC-TATTCACT--TAT------AAACAC-------AA----AGT-CTATGAATG----TA CC-TATTCACT--TAT----_-AAACAC----_--AA----AGT-CTATGAATG----TA CС-TATTCACT-OTAT--CC-TACACT-TATCC-TATCACT TA

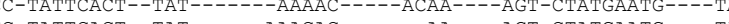
CC-TATWCACT-TAT---DAACAC--0--AA----AGT-CTATGAATG----T CC-TATICACT--TAT---_-AAACAC---1--AA--AGT-CTATGAATG----TA CC-TATTCACT--TAT-----AAACAC------AA----AGT-CTATGAATG----TA CC-TATTCACT--TAT------AAACAC-------AA----AGT-CTATGAATG----TA CC-TATTCACT--TAT------AAACAC-------AA----AGT-CTATGAATG----TA CC-TATTCACT--TAT------AAACAC-------AA----AGT-CTATGAATG----TA CC-TATTCACT--TAT------AAACAC-------AA----AGT-CTATGAATG----TA CC-TATTCACT--TAT------AAACAC-------AA----AGT-CTATGAATG----TA CGGTCCTCTGT--TATCCTAGCAAGTACTTTACATAACCCCATTCCTTTACATGAGATGA CGGTCCTCTGT- TATCCTAGCAAGTACTTTACATARCCCCATCCTTTACATGAGATGA TCTATAC----ACAACCCCATGCTTTACATGAGATG TGCTATCAAGT-TGCTATCAAGT-AT---CTITAC----ACAACCCCATMGITIACATGAGATGA IGCTATCAAGT---AT------CTTTAC-----ACAACCCCATTGCTTTACATGAGATGA AT------AGT---AT------AAGAAT-----GTAC---CAGT-CTCTTAAT------TT------AGT---AT------AAGAAT-----GTAC---CAGT-CTCTTAAT-----AC-----AGT---AT------AAGAAT-----GTAC---CAGT-CTCTTAAT-----AT-----AGT---AT------AAGAAT-----GTACC---AGT-CTCTTAAT--------TATAAAGT--CATT------AGAAT-----GTAAA--CAGT-CTCTTAAT-----TA----AAAGT---CT------AAGAAT-----GTAAAA-CAGT-CTCTTAAG---------ATAAAGT---CT------AAGAAT-----GTAAAA-CAGT-CTCTTAAC-----TTTT-TAACAAGT --CT----oTT-TAACAAGT---CT------AAGAAT-----GTAAAACCAGT-CTCTTAAC------

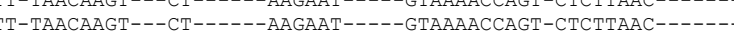
TT-TAACAAGT---CT------AAGAAT-----GTAAAACCAGT-CTCTTAAC-----AT------AGT---AT------AAGAAT-----GTAAC---AGT-CTCTTAAT-----AT------AGT---AT------AAGAAT-----GTAAC---AGT-CTCTTAAT------TT-----AGT---AT-----AAGAAT-----GTAAC---AGT-CTCTTAAT----TT------AGT---AT------AAGAAT-----GTAA---CAGT-CTCTTAAT------TT------AGT---AT------AAGAAT-----GTAAC---AGT-CTCTTAAT------

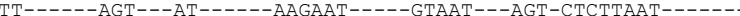
TIT-TTTT------AGT---AT------AAGAAT-----GTAAT---AGT-CTCTTAAT-----TT-----AGT---AT-----AAGAAT-----GTAA---TAGT-CTCTTAAT-----TT------AGT---AT------AAGAAT-----GTAA---TAGT-CTCTTAAT-----TT-----AGT---AT------AAGAAT-----GTAA---TAGT-CTCTTAAT-----TT------AGT---AT------AAGAAT-----GTAAT---AGT-CTCTTAAT-----TT-----AGT---AT------AAGAAT-----GTAA---TAGT-CTCTTAAT----TT------AGT---AT------AAGAAT-----GTAA---TAGT-CTCTTAAT-----TT-----AGT---AT-----AAGAAT-----GTAA---TAGT-CTCTTAAT-----TT------AGT---AT------AAGAAT-----GTAA---TAGT-CTCTTAAT-----TT------AGT---AT------AAGAAT-----GTAA---TAGT-CTCTTAAT------TT------AGT---AT------AAGAAT-----GTAA---TAGT-CTCTTAAT------TT------AGT---AT------AAGAAT-----GTAA---TAGT-CTCTTAAT------ 
C. chernovii FN400936 C. magnus AF190009 C. oeirensis AF444364 E5S-PDA
E3M-STD

Fungal sp. GU213437

R. mucilaginosa AF444614 25S-2-PDA

R. mucilaginosa AF444609

R. mucilaginosa AF444503

R. mucilaginosa CBS2382

R. mucilaginosa FR690147

R. mucilaginosa HQ702343

R. mucilaginosa FJ515212

R. mucilaginosa EU149812

R. mucilaginosa EU871492

Uncultured fungus FM875856

R. mucilaginosa CBS8618

R. mucilaginosa AF444635

K. malvinella AF444498

K. malvinella D0667165

K. aeria AM922224

K. aeria AF444562

K. aeria AF444675 H10S-PDA

Basidiomycete BSU6561

R. sp. FJ238090

R. sp. FJ 865356

P2S-PDA

R. oryzicola AY335160

R. sp. FJ008055

S. dacryoidea AF444571 24S-1-PDA

S. dacryoidea AF444597

S. dacryoidea AF 444500

S. dacryoidea AB049027

S. dacryoidea DQ832207

R. minuta AB078490

R. minuta AB078484

R. minuta $A B 038117$ B 4M-STD

R. minuta $A B 078501$

R. minuta AF190010

R. minuta AF190010

R. minuta AB078493
R. minuta AB078497 I1-STD

R. $\mathrm{sp}$. AF444619

R. slooffiae FJ515215

R. slooffiae AB025994 J3S-STD

R. Slooffiae FJ807685

R. Slooffiae AF444589

R. Slooffiae AB078491

R. slooffiae AB052212

R. slooffiae AF444627

R. Slooffiae AF444552

R. Slooffiae AF 444552

R. Slooffiae AB078495
R. slooffiae CBS8411
TAGTCTTATTATAACA--_----TAATAA--AACTTTTAACAACGGATCTCTTGGCTCTC ACTCTTATMAACA TAGTCTTATTATAACA-------TAATAA--AACTTTCAACAACGGATCTCTTGGCTCTC TTAAATTTT-ATAACA------AAATAA--AACTTTCAACAACGGATCTCTTGGCTCTC TTAAATTTT-ATAACA-------AAATAA--AACTTTCAACAACGGATCTCTTGGCTCTC TTAAATTTT-ATAACA------AAATAA--AACTTTCAACAACGGATCTCTTGGCTCTC TTAAATTTT-ATAACA-------AAATAA--AACTTTCAACAACGGATCTCTTGGCTCTC TTAAATTTT-ATAACA-------AAATAA--AACTTTCAACAACGGATCTCTTGGCTCTC TTAAATTT-ATAACA---0-AMATAR--AACTTTCAACAACGGATCTCTTCGCTCTC

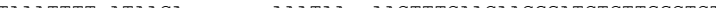
TAAATTTT-ATAACA--TTAAATTTT-ATAACA-------AAATAA--AACTTTCAACAACGGATCTCTTGGCTCTC TTAAATTTT-ATAACA------AAATAA--AACTTTCAACAACGGATCTCTTGGCTCTC TTAAATTTT-ATAACA------AAATAA--AACTTTCAACAACGGATCTCTTGGCTCTC TTAAATTTT-ATAACA------AAATAA--AACTTTCAACAACGGATCTCTTGGCTCTC TTAAATTTT-ATAACA-A-----AATAA--AACTTTCAACAACGGATCTCTTGGCTCTC TTAAATTTT-ATAACA------AAATAA--AACTTTCAACAACGGATCTCTTGGCTCT TTAAATTTT-ATAACA-------AAATAA--AACTTTCAACAACGGATCTCTTGGCTCTC TGATGTTAC-ATAATTTGAAATCAAAGAAACAACTTTCAGCAACGGATCTCTTGGCTCTC TCATCTTAC-ADA TGATGTTAC-ATAATTTGAAATCAAAGAAACAACTTTCAGCAACGGATGTCTTGGCTCTC TGATGTTAC-ATAATTTGAAATGAAAGAAACAACTTTCAGCAACGGATGTCTTGGCTCTC TGATGTTAC-ATAATTTGAAATGAAAGAAACAACTTTCAGCAACGGATGTCTTGGCTCTC

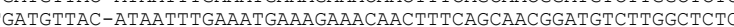
TGAGCATAA-ATATAT-------AATAA--AACTTTCAGCAACGGATCTCTTGGCTCTC TGAGCATAA-ATATAT-------AATAA--AACTTTCAGCAACGGATCTCTTGGCTCTC TGAGCATAA-ATATAT-------AATAA--AACTTTCAGCAACGGATCTCTTGGCTCTC TGAGCATAA-ATATAT-------AATAA--AACTTTCAGCAACGGATCTCTTGGCTCTC TGAGCATAAACTATA---------ACAA--AACTTTCGGCAACGGATCTCTTGGCTCTC TGAGCATAA-ACAT---------AAACAA--AACTTTCAGCAACGGATCTCTTGGCTCTC

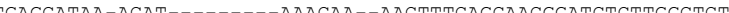
TCTTGGCTCT TGAGCATAA-ACAT--.------AAACAA--AACTTTCAGCAACGGATCTCTTGGCTCTC GAGCATAA-ACAT---------AAACAA--AACTTTCAGCAACGGATCTCTTGGCTCTC TGAGCATAA-ACAT-------AAACAA--AACTTTCAGCAACGGATCTCTTGGCTCTC TGAGCATAA-ACAT-------AAACAA--AACTTTCAGCAACGGATCTCTTGGCTCTC TGAGCATAA-ATAA-A-------AATAA--AACTTTCAGCAACGGATCTCTTGGCTCTC TGAGCATAA-ATAA-A------AATAA--AACTTTCAGCAACGGATCTCTTGGCTCTC TGAGCATAA-ATAA-A-------AATAA--AACTTTCAGCAACGGATCTCTTGGCTCTC TGAGCATAA-ATAAA--------AATAA--AACTTTCAGCAACGGATCTCTTGGCTCTC TGAGCATAA-ATAA-A--------AATAA--AACTTTCAGCAACGGATCTCTTGGCTCTC

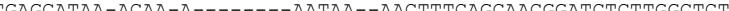
作 CAARTGAGCATAA-ACAA-A-A GAGCATAA-ATAAA------AACAA--AACTTTCAGCAACGGATCTCTTGGCTCTC TGAGCATAA-ATAA-------AAACAA--AACTTTCAGCAACGGATCTCTTGGCTCT TGAGCATAA-ATAA--------AAACAA--AACTTTCAGCAACGGATCTCTTGGCTCTC TGAGCATAA-ATAA--------AAACAA--AACTTTCAGCAACGGATCTCTTGGCTCTC TGAGCATAA-ATAAA-------AACAA--AACTTTCAGCAACGGATCTCTTGGCTCTC TGAGCATAA-ATAA-------AAACAA--AACTTTCAGCAACGGATCTCTTGGCTCTC TGAGCATAA-ATAA--------AAACAA--AACTTTCAGCAACGGATCTCTTGGCTCTC TGAGCATAA-ATAA---------AAACAA--AACTTTCAGCAACGGATCTCTTGGCTCTC TGACCATAA-ATAA-------AAACAA--AACTTTCAGCAACGGATCTCTTGGCTCTC TCA TGACCATAA-ATA---

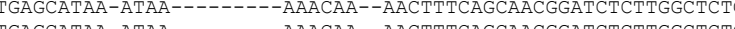
TGAGCATAA-ATAA--------AAACAA--AACTTTCAGCAACGGATCTCTTGGCTCTC 
C. chernovii FN400936 C. magnus AF190009

C. oeirensis AF444364 5S-PDA E3M-STD

Fungal sp. GU213437

R. mucilaginosa AF444614 Q5S-2-PDA

R. mucilaginosa AF444609

R. mucilaginosa AF444503

R. mucilaginosa CBS2382

R. mucilaginosa FR690147

R. mucilaginosa HQ702343
R. mucilaginosa FJ515212

R. mucilaginosa EU149812

R. mucilaginosa EU871492

Uncultured fungus FM875856

R. mucilaginosa CBS8618

R. mucilaginosa AF444635

K. malvinella AF444498

K. aeria AM922224

K. aeria AF444562

K. aeria AF444675

H10S-PDA

Basidiomycete BSU65613

R. sp. FJ238090

R. sp. FJ865356

P2S-PDA

R. oryzicola AY335160

R. sp. FJ008055

S. dacryoidea AF444571 Z4S-1-PDA

S. dacryoidea AF444597

S. dacryoidea AF444500

S. dacryoidea AB049027

S. dacryoidea DQ832207

. minuta AB078490

R. minuta $A B 078484$

R. minuta $A B 038117$

B 4M-STD

R. minuta $A B 078501$

R. minuta AF190010

R. minuta AF190010

R. minuta AB078493
R. minuta AB078497

I1-STD

R. $\mathrm{sp}$. AF444619

R. Slooffiae FJ515215

R. slooffiae AB025994

J3S-STD

R. Slooffiae FJ807685

R. slooffiae AF444589

R. slooffiae AB078491

R. slooffiae AB052212

R. slooffiae AF444627

R. Slooffiae AF444552

R. Slooffiae AF444552

R. Slooffiae AB078495
R. slooffiae CBS8411
CAMCGATGAAGAACGCAGCGAAATGCGATAAGTAATGTGAATTGCAGAATTCAGTGAAT CATCGATGAAGAACGCAGCGAAATGCGATAAGTAATGTGAATTGCAGAATTCAGTGAA GCATCGATGAAGAACGCAGCGAAATGCGATAAGTAATGTGAATTGCAGAATTCAGTGAAT GCATCGATGAAGAACGCAGCGAAATGCGATAAGTAATGTGAATTGCAGAATTCAGTGAA GCATCGATGAAGAACGCAGCGAAATGCGATAAGTAATGTGAATTGCAGAATTCAGTGAAT GCATCGATGAAGAACGCAGCGAAATGCGATAAGTAATGTGAATTGCAGAATTCAGTGAAT GCATCGATGAAGAACGCAGCGAAATGCGATAAGTAATGTGAATTGCAGAATTCAGTGAAT CATCGATGAAGAACGCAGCGAAATGCGATAAGTAATGTGAATTGCAGAATTCAGTGAAT CATCGATCAAGACCCAGCGAATCCGATAAGTAATCTCAATTCCAGAATTCAGTGABT CCATCGA 作 GGATGAAGAACGCAGCGAAATGCGATAAGTAATGTGAATTGCAGAATTCAGTGAA GCATCGATGAAGAACGCAGCGAAATGCGATAAGTAATGTGAATTGCAGAATTCAGTGAAT GCATCGATGAAGAACGCAGCGAAATGCGATAAGTAATGTGAATTGCAGAATTCAGTGAAT GCATCGATGAAGAACGCAGCGAAATGCGATAAGTAATGTGAATTGCAGAATTCAGTGAAT GCATCGATGAAGAACGCAGCGAAATGCGATAAGTAATGTGAATTGCAGAATTCAGTGAAT GCATCGATGAAGAACGCAGCGAAATGCGATAAGTAATGTGAATTGCAGAATTCAGTGAAT GCATCGATGAAGAACGCAGCGAAATGCGATAAGTAATGTGAATTGCAGAATTCAGTGAAT GCATCGATGAAGAACGCAGCGAAATGCGATAAGTAATGTGAATTGCAGAATTCAGTGAAT CCATCGATGAAGAACGCAGCGAAATGCGATACGTAATGTGAATTCCAGAACTCAGTGAAT CCATCCATCAACAACGCACCAATCCATACCTAATT

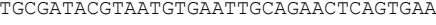

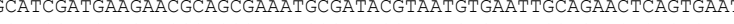
GCATCGATGAAGA GCATCGATGAAGAACGCAGCGAAATGCGATACGTAATGTGAATTGCAGAACTCAGTGAAT GCATCGATGAAGAACGCAGCGAATTGCGATAAGTAATGTGAATTGCAGAATTCAGTGAAT GCATCGATGAAGAACGCAGCGAATTGCGATAAGTAATGTGAATTGCAGAATTCAGTGAAT GCATCGATGAAGAACGCAGCGAATTGCGATAAGTAATGTGAATTGCAGAATTCAGTGAAT GCATCGATGAAGAACGCAGCGAATTGCGATAAGTAATGTGAATTGCAGAATTCAGTGAAT CCATCGATGAAGAACGCAGCGAAATGCGATACGTAATGTGAATTGCAGAATTCAGTGAAT CCATCGATGAAGAACGCAGCGAAATGCGATACGTAATGTGAATTGCAGAATTCAGTGAAT CCATCGATGAACAACGCAGCGAATGCGATACGTAATGTGAATTCCAGAATTCAGTGATT CCATCG TGAGACGCAGCGAAAGCGAACGTATGTGAATGCAGAATCAGTGAT

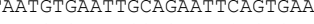
CCATCGATGAAGAACGCAGCGAAATGCGATACGTAATGTGAATTGCAGAATTCAGTGAAT CCATCGATGAAGAACGCAGCGAAATGCGATACGTAATGTGAATMGCAGAATMCAGTGAA CCATCGATGAAGAACGCAGCGAAATGCGATACGTAATGTGAATTGCAGAATTCAGTGAAT GCATCGATGAAGAACGCAGCGAATTGCGATAAGTAATGTGAATTGCAGAATTCAGTGAAT GCATCGATGAAGAACGCAGCGAATTGCGATAAGTAATGTGAATTGCAGAATTCAGTGAAT GCATCGATGAAGAACGCAGCGAATTGCGATAAGTAATGTGAATTGCAGAATTCAGTGAAT GCATCGATGAAGAACGCAGCGAATTGCGATAAGTAATGTGAATTGCAGAATTCAGTGAAT GCATCGATGAAGAACGCAGCGAATTGCGATAAGTAATGTGAATTGCAGAATTCAGTGAAT GCATCGATGABGAACGCAGCGAATGCGATAAGTAATGTGAATTCCAGAMTCAGTGAAT GCACATGA

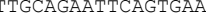
GATCGATGAAGAACGCAGCGAATTGCGATAAGTAATGTGAATTGCAGAATTCAGTGAA GCATCGATGAAGAACGCAGCGAATTGCGATAAGTAATGTGAATTGCAGAATTCAGTGAA GCATCGATGAAGAACGCAGCGAATTGCGATAAGTAATGTGAATTGCAGAATTCAGTGAAT GCATCGATGAAGAACGCAGCGAATTGCGATAAGTAATGTGAATTGCAGAATTCAGTGAAT GCATCGATGAAGAACGCAGCGAATTGCGATAAGTAATGTGAATTGCAGAATTCAGTGAAI GCATCGATGAAGAACGCAGCGAATTGCGATAAGTAATGTGAATTGCAGAATTCAGTGAAT GCATCGATGAAGAACGCAGCGAATTGCGATAAGTAATGTGAATTGCAGAATTCAGTGAAT GCATCGATGAAGAACGCAGCGAATTGCGATAAGTAATGTGAATTGCAGAATTCAGTGAAT GCATCGATGAAGAACGCAGCGAATTGCGATAAGTAATGTGAATTGCAGAATTCAGTGAAT GCATCGATGAAGAACGCAGCCAATTCCCATAAGTAATGTGAATTCCACAATTCAGTCAAT CCACG CCACGATGAGA CCCACCGATHGCGATAGTAATGIGAATTCCAGATCAGTGAA GCATCGATGAAGAACCAGCCARTTCGATAAGA GCATCGATGAAGAACGCAGCGAATTGCGATAAGTAATGTGAATTGCAGAATTCAGTGAAT 
C. chernovii FN400936 C. magnus AF190009

C. oeirensis AF44436 E5S-PDA
E3M-STD

Fungal sp. GU213437

R. mucilaginosa AF44461 Q5S-2-PDA

R. mucilaginosa AF444609

R. mucilaginosa AF444503

R. mucilaginosa CBS2382

R. mucilaginosa FR690147

R. mucilaginosa HQ702343

R. mucilaginosa FJ515212

R. mucilaginosa EU149812

R. mucilaginosa EU871492

Uncultured fungus FM875856

R. mucilaginosa CBS 8618

R. mucilaginosa AF444635

K. malvinella AF444498

K. aeria AM922224

K. aeria AF444562

K. aeria AF444675

H10S-PDA

Basidiomycete BSU6561

R. sp. FJ238090

R. sp. FJ865356

P2S-PDA

R. oryzicola AY335160

R. sp. FJ008055

S. dacryoidea AF444571

$\mathrm{Z} 4 \mathrm{~S}-1-\mathrm{PDA}$

S. dacryoidea AF444597

S. dacryoidea AF444500

S. dacryoidea AB049027

S. dacryoidea DQ832207

R. minuta $A B 078490$

R. minuta $A B 078484$

R. minuta $A B 038117$

B 4M-STD

R. minuta $A B 078501$

R. minuta AF190010

R. minuta AF190010

R. minuta AB078493
R. minuta AB078497

I1-STD

R. sp. AF444619

R. Slooffiae FJ515215

R. slooffiae AB025994 J3S-STD

R. Slooffiae FJ807685

R. slooffiae AF444589

R. slooffiae AB078491

R. slooffiae AB052212

R. slooffiae AF444627

R. Slooffiae AF444552

R. Slooffiae AF444552

R. Slooffiae AB078495
R. slooffiae CBS8411
СATCGAATCTTTGAACGCACCTTGCGCTCCTTGG-TATTCC---GAGGAGCATGCCTGTT ATCGAATCTTTGAACGCACCTTGCGCTCCTTGG-TATTCC---GAGGAGCATGCCTGT CATCGAATCTTTGAACGCACCTTGCGCTCCTTGG-TATTCC----GAGGAGCATGCCTGT CATCGAATCTTTGAACGCACCTTGCGCTCCATGG-TATTCC---GTGGAGCATGCCTGT CATCGAATCTTTGAACGCACCTTGCGCTCCATGG-TATTCC---GTGGAGCATGCCTGT CATCGAATCTTTGAACGCACCTTGCGCTCCATGG-TATTCC---GTGGAGCATGCCTGT CATCGAATCTTTGAACGCACCTTGCGCTCCATGG-TATTCC---GTGGAGCATGCCTGTI CATCGAATCTTTGAACGCACCTTGCGCTCCATGG-TATTCC---GTGGAGCATGCCTGTT CATCGA

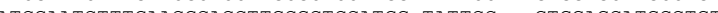
作 作 ATCGAATCTTTGAACGCACCTTGCGCTCCATGG-TATTCC---GTGGAGCATGCCTGTT CATCGAATCTTTGAACGCACCTTGCGCTCCATGG-TATTCC---GTGGAGCATGCCTGT CATCGAATCTTTGAACGCACCTTGCGCTCCATGG-TATTCC---GTGGAGCATGCCTGTT CATCGAATCTTTGAACGCACCTTGCGCTCCATGG-TATTCC---GTGGAGCATGCCTGT CATCGAATCTTTGAACGCACCTTGCGCTCCATGG-TATTCC---GTGGAGCATGCCTGTT CATCGAATCTTTGAACGCACCTTGCGCTCCATGG-TATTCC---GTGGAGCATGCCTGTT CATCGAATCTTTGAACGCACCTTGCGCTCCATGG-TATTCC---GTGGAGCATGCCTGTT CATCGATTTTTGAACGCACCTTCCGCTCTTTAGTTATTCT--CAACACCATCCTTCTI CATCG CATCGAATTTTTGA CGCACCTTGCGCTCTTTAGTTATTCT---GAAGAGCATGCTTGT CATCGAATTTTTGAACGCACCTIGCGCTCTTTAGTTATTCT---GAAGAGCATGCTTGT CAICGAATTITGAACGCACCIIGCGCTCTTTAGTTATTCT---GAAGAGCATGCTTGT CATCGAATTTTTGAACGCACCTTGCGCTCTTTAGTTATTCT---GAAGAGCATGCTTGTI CATCGAATCTTTGAACGCACCTTGCACTCTTTGG-TATTCC---GAAGAGTATGTCTGTT CATCGAATCTTTGAACGCACCTTGCACTCTTTGG-TATTCC---GAAGAGTATGTCTGTT CATCGAATCTTTGAACGCACCTTGCACTCTTTGG-TATTCC---GAAGAGTATGTCTGTT CATCGAATCTTTGAACGCACCTTGCACTCTTTGG-TATTCC---GAAGAGTATGTCTGTT CATCGAATCTTTGAACGCACCTTGCACTCTTTGG-TATTCC---GAAGAGTATGTCTGTT CATCGAATCTTTGAACGCACCTTGCACTCTTTGG-TATTCC---GAAGAGTATGTCTGTT CATCGA CATCG CAICGAATCTITGAACGCACCIT CATCGAATCTTTGAACGCACCTTGCACTCTTTGG-TATTCC---GAAGAGTATGTCTGTT CATCGAATCTTTGAACGCACCTTGCACTCTTTGG-TATTCC---GAAGAGTATGTCTGTT CATCGAATCTTTGAACGCACCTTGCACTCTTTGG-TATTCC---GAAGAGTATGTCTGT CATCGAATCTTTGAACGCACCTTGCACTCTTTGG-TATTCC---GAAGAGTATGTCTGT CATCGAATCTTTGAACGCACCTTGCACTCTTTGG-TATTCC---GAAGAGTATGTCTGTT CATCGAATCTTTGAACGCACCTTGCACTCTTTGG-TATTCC---GAAGAGTATGTCTGTT CATCGAATCTTTGAACGCACCTTGCACTCTTTGG-TATTCC---GAAGAGTATGTCTGTT CATCCAATCTTTGABCGCACCTTCCACTCTTTCG-TATTCC--

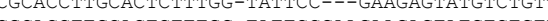

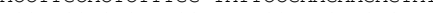
CATCGAATCTITGAACGCACCITGCACTCTIGO-TATHCL--GAAGAGTATGTCTGTT CATCGAATCTTTGAACGCACCTTGCACTCTTTGG-TATTCC---GAAGAGTATGTCTGT CATCGAATCTTTGAACGCACCTTGCACTCTITGG-TATTCC---GAAGAGTATGTCTGT CATCGAATCTTTGAACGCACCTTGCACTCTTTGG-TATTCC---GAAGAGTATGTCTGTT CATCGAATCTTTGAACGCACCTTGCACTCTTTGG-TATTCC---GAAGAGTATGTCTGT CATCGAATCTTTGAACGCACCTTGCACTCTTTGG-TATTCC---GAAGAGTATGTCTGT CATCGAATCTTTGAACGCACCTTGCACTCTTTGG-TATTCC---GAAGAGTATGTCTGTT CATCGAATCTTTGAACGCACCTTGCACTCTTTGG-TATTCC---GAAGAGTATGTCTGTT CATCGAATCTTTGAACGCACCTTGCACTCTTTGG-TATTCC---GAAGAGTATGTCTGTC CATCGAATCTTTCAACGCACCTTCCACTCTTTGG-TATTCC--CAACAGTATGTCTGTI

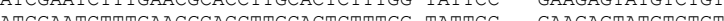
CATCGA TCTITGACGCACCTCCACTCTIGG-TATCC--GAAGAGTATGICIGT CATCGAATCTTTGAACGCACCTTGCACTCTTTGG-TATTCC---GAAGAGTATGTCTGTT 
C. chernovii FN400936 C. magnus AF19000

C. oeirensis AF444364 E5S-PDA
E3M-STD

Fungal sp. GU213437

R. mucilaginosa AF44461 Q5S-2-PDA

R. mucilaginosa AF444609

R. mucilaginosa AF444503

R. mucilaginosa A 444503

R. mucilaginosa FR690147

R. mucilaginosa HQ702343

R. mucilaginosa FJ515212

R. mucilaginosa EU149812

R. mucilaginosa EU871492

Uncultured fungus FM875856

R. mucilaginosa CBS8618

R. mucilaginosa AF444635

K. malvinella AF444498

K. malvinella DQ667165

K. aeria AM922224

K. aeria AF444562

K. aeria AF444675

H10S-PDA

Basidiomycete BSU6561

R. sp. FJ238090

R. sp. FJ865356

R. oryzicola $A$ 3 335160

R.

S. dacryoidea AF444571 Z4S-1-PDA

S. dacryoidea AF444597

S. dacryoidea AF444500

S. dacryoidea AB049027

S. dacryoidea DQ832207

R. minuta $A B 078490$

R. minuta AB078484

R. minuta $A B 038117$

B 4M-STD

R. minuta $A B 078501$

R. minuta AF190010

R. minuta AF190010

R. minuta AB078493

I1-STD

R. $\mathrm{sp}$. AF444619

R. slooffiae FJ515215

R. slooffiae AB025994

J3S-STD

R. Slooffiae FJ807685

R. Slooffiae AF444589

R. Slooffiae AB078491

R. slooffiae AB052212

R. slooffiae AF444627

R. Slooffiae AF444552

R. Slooffiae AB078495

R. Slooffiae AB078495
R. slooffiae CBS8411
TGAGTGTCATGAA-ACTCTCAAA-CCCAAGT---TTTGGATTTCGATCCATGCT----TG TGAGTGTCATGAA-ACCCTCAAA-CCCAAGT---TTTGGATTTCGATCCATGCT----TG IGAGTGTCATGAA-ACCCTCAAA-CCCAAGT---TTTGGATTTCGGTCCATGCT----TG TGAGTGTCATGAATACT-TCAA--CCCTCCTCT-TTCTTAA-TGA-TTGAAGCGGT-GTTGAGTGTCATGAATACT-TCAA--CCCTCCTCT-TTCTTAA-TGA-TTGAAGAGGT-GTTGAGTGTCATGAATACT-TCAA--CCCTCCTCT-TTCTTAA-TGA-TTGAAGAGGT-GTTGAGTGTCATGAATACT-TCAA--CCCTCCTCT-TTCTTAA-TGA-TTGAAGAGGT-GTTGAGTGTCATGAATACT-TCAA--CCCTCCTCT-TTCTTAA-TGA-TTGAAGAGGT-GTTCAGTCTCATCAATACT-TCAA--CCCTCCTCT-TTCTTAA-TCA-TTGARCACCT-GT-

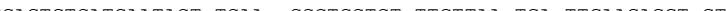

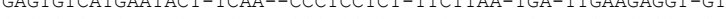
TGAGTGTCATGAATACT-TCAA-CCCTCCTCT-TMCTTAA-TGA-TTGAAGAGGT-GTIGAGTGTCATGAATACT-TCAA--CCCTCCTCT-TTCTTAA-TGA-TTGAAGAGGT-GT TGAGTGTCATGAATACT-TCAA--CCCTCCTCT-TTCTTAA-TGA-TTGAAGAGGT-GTTGAGTGTCATGAATACT-TCAA--CCCTCCTCT-TTCTTAA-TGA-TTGAAGAGGT-GTTGAGTGTCATGAATACT-TCAA--CCCTCCTCT-TTCTTAA-TGA-TTGAAGAGGT-GTTGAGTGTCATGAATACT-TCAA--CCCTCCTCT-TTCTTAA-TGA-TTGAAGAGGT-GTTGAGTGTCATGAATACT-TCAA--CCCTCCTCT-TTCTTAA-TGA-TTGAAGAGGT-GTTGAGTGTCATGAATACT-TCAA--CCCTCCTCT-TTCTTAA-TGA-TTGAAGAGGT-GTTGAGTGTCGCGAAC-CTCTCAAC-CCCGCCAAAAGCTTTAC-TGACTTTGGTTGTT--TG TGAGTGTCGCGAA-CCTCTCAAC-CCCCCCAAAGCCTTTAC-TGACTTTGGTTGTT--TG TGAGTGTCGCGA-CCTCTCAAC TG TGATCTIT

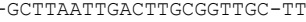
TGAGTGTCGCGAA-CCTCTCAACCCCGCCCAA--GGCTTAATTGACTTGCGGTTGT-TTG TGAGTGTCATGAA-ACTCTCAA--CCCCCCTGT-TTTGTAA-CGA---GATAGGC--GT TGAGTGTCATGAA-ACTCTCAA--CCCCCCTGT-TTTGTAA-TGA---GATAGGC--GTG TGAGTGTCATGAA-ACTCTCAA--CCCCCCTGT-TTTGTAA-TGA---GACAGGC--GTG TGAGTGTCATGAA-ACTCTCAA--CCCCCCTGT-TTTGTAA-TGA---GACAGGC--GTG TGAGTGTCATGAA-ACTCTCAA---CCTCCTTGTTTTGTAA-TGAA---ACGAGTT-GTG TGAGTGTCATGAA-ACTCTTAA--CCCCCTTGT-TTTGTAA-TGAA---ACGAGTT-CTG TCAGTGTCATGAA-ACTCTCA-OCCCCTTGT-TTTGTAA-TGAA---ACGACC-OTG C. ACTCTCAA-CCCCCTGT-TTTGTAA-TGA--GACAAGC--GT TGAGTGTCATGAA-ACTCTCAA--CCCCCTTGT-TTIGTAA-TGA---GACAAGC--GTG

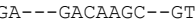
TGAGTGTCATGAA-ACTCTCAA--CCCCCTTGT-TTTGTAA-TGA---GACAAGC--GTG TGAGTGTCATGAA-ACTCTCAA--CCCCCTTAT-TTTGTAA-TGA---GATAAGT--GCG TGAGTGTCATGAA-ACTCTCAA--CCCCCTTAT-TTTGTAA-TGA---GATAAGT--GCG TGAGTGTCATGAA-ACTCTCAA--CCCCCCTAT-TTTGTAA-TGA---AATGGGC--GTG TGAGTGTCATGAA-ACTCTCAA--CCCCCCTAT-TTTGTAA-TGA---GATGGGT--GTG TGAGTGTCATGAA-ACTCTCAA--CСССССТАT-TTTGTAA-TGA---GATGGGT--CT TCAGTATCATGAA-ACTCTCAA-CCCCCCTAT-TTTGTAA-TGA--UATGGGC-GTG

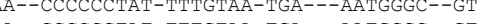
CCCCCCIAT-TIGTAA-TGA GAGTATCATGAA-ACTCTCAA--CCCCCCTAT-ITTGTAA-TGA---AATGGGC--GT 作 GATGTCATGAA-ACICTCAA--CCCCCCTAI-ITIGTAA-TGA---GATGGGC--GT TGAGTGTCATGAA-ACTCTCAA--CCCCCCTAT-TTTGTAA-TGA---GATGGGC--GTG TGAGTGTCATGAA-ACTCTCAA--CCCCCCTAT-TTTGTAA-TGA---GATGGGC--GTG TGAGTGTCATGAA-ACTCTCAA--CCCCCCTAT-TTTGTAA-TGA---GATGGGT--GTG TGAGTGTCATGAA-ACTCTCAA--CCCCCCTAT-TTTGTAA-TGA---GATGGGT--GTG TGAGTGTCATGAA-ACTCTCAA--CCCCCCTAT-TTTGTAA-TGA---GATGGGT--GTG TGAGTGTCATGAA-ACTCTCAA--CCCCCCTAT-TTTGTAA-TGA---GATGGGT--CTG TGAGTGTCATCAA-ACTCTCAA--ССССССТАT-TTTGTAA-TGA---GATGCGT--GTG TGAGTCACATC TGATGGT-GT IGAGIGICATGAA-ACICICAA-CCCCCCTAT-TA-T TGAGTGTCATGAA-ACTCTCAA--CCCCCCTAT-TTTGTAA-TGA---GATGGGT--GTG
TGAGTGTCATGAA-ACTCTCAA--CCCCCCTAT-TTTGTAA-TGA---GATGGGT--GTG 
C. chernovii FN400936 C. magnus AF190009

C. oeirensis AF444364 $5 S-P D A$ E3M-STD

Fungal sp. GU213437

R. mucilaginosa AF444614 Q5S-2-PDA

R. mucilaginosa AF444609 R. mucilaginosa AF444503

R. mucilaginosa CBS2382

R. mucilaginosa FR690147

R. mucilaginosa HQ702343

R. mucilaginosa FJ515212

R. mucilaginosa EU149812

R. mucilaginosa EU871492

Uncultured fungus FM875856

R. mucilaginosa CBS8618

R. mucilaginosa AF444635

K. malvinella AF444498

K. aeria AM922224

K. aeria AF444562

K. aeria AF444675

H1OS-PDA

Basidiomycete BSU6561

R. sp. FJ238090

R. sp. FJ865356

R. oryzicola $A Y 335160$

R. SP.

S. dacryoidea AF444571 Z4S-1-PDA

S. dacryoidea AF444597

S. dacryoidea AF444500

S. dacryoidea AB049027

S. dacryoidea DQ832207

R. minuta AB078490

R. minuta $A B 078484$

R. minuta $A B 038117$

B 4M-STD

R. minuta $A B 078501$

R. minuta AF190010

R. minuta AF190010

R. minuta AB078493
R. minuta AB078497

I1-STD

R. sp. AF444619

R. Slooffiae FJ515215

R. slooffiae AB025994

J3S-STD

R. Slooffiae FJ807685

R. Slooffiae AF444589

R. slooffiae AB078491

R. slooffiae AB052212

R. slooffiae AF444627

R. Slooffiae AF444552

R. Slooffiae AF444552

R. Slooffiae AB078495
R. slooffiae CBS8411
AGTTTGCATT-TGGATGTTTGCCCG-----GTGATAAGCC-СACTCATCTTAAAAGTATT GTTTGGATT-TGGATGTTTGCCGG-------TGATGAACCGACTCATCTTAAAAGTATT AGTTTGGATT-TGGACGTTTGCCGG-------TGATGAACCGACTCGTCTTAAAAGTATT ---TTGGTTTCTGAGCG-CTGCTGG---CCTTTACGGTCTAG-CTCGTTCGTAATGCATI ---TTGGTTTCTGAGCG-CTGCTGG---CCTTTACGGTCTAG-CTCGTTCGTAATGCATT ---TTGGTTTCTGAGCG-CTGCTGG---CCTTTACGGTCTAG-CTCGTTCGTAATGCATT ---TTGGTTTCTGAGCG-CTGCTGG---CCTTTACGGTCTAG-CTCGTTCGTAATGCATT ---TTGGTTTCTGAGCG-CTGCTGG---CCTTTACGGTCTAG-CTCGTTCGTAATGCATI -

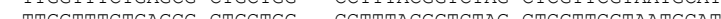
--TTGGTTTCTGAGCG-CTGCTGG---CCTTTACGGTCTAG-CTCGTTCGTAATGCAT --TTGGTTTCTGAGCG-CTGCTGG---CCTTIACGGTCTAG-CTCGTTCGTAATGCATI --TTGGTTTCTGAGCG-CTGCTGG---CCTTTACGGTCTAG-CTCGTTCGTAATGCAT ---TTGGTTTCTGAGCG-CTGCTGG---CCTTTACGGTCTAG-CTCGTTCGTAATGCATT ---TTGGTTTCTGAGCG-CTGCTGG---CCTTTACGGTCTAG-CTCGTTCGTAATGCATT ---TTGGTTTCTGAGCG-CTGCTGG---CCTTTACGGTCTAG-CTCGTTCGTAATGCATT ---TTGGTTTCTGAGCG-CTGCTGG---CCTTTACGGTCTAG-CTCGTTCGTAATGCATT GGCTTGGATCATGCTCTCTTGTCGTTACCTTTCGGGGTTTGGACTGGACTGAAATACABC GGCTTCGATCATGCTCTCTTCTCGTTACCTTTCGCGGTTTGGACTGCACTCAAATACAAC 作 GCTTGGATCATGGTCTCTTGTCGITACCIT-CGGGTITGGACTGGACTGAAATACAAC GGCTIGGATCATGGTCTCTTGTCGITACCTI--CGGGIITGGACTGGACTGAAATACAAC GGCTTGGATCATGGTCTCTTGTCGTTACCTT--CGGGTTTGGACTGGACTGAAATACAAC GGCTTGGATTATGGTTGTCTGTCGG---CGT--AAAGTC--GGCTCAACTGAAATATACG GGCTTGGATTATGGCTGTCTGTCGG---CGT--AAAGTC--GGCTCAGCTGAAATACACG GGCTTGGATTATGGCTGCCTGTCGG---CGT--AAAGTC--GGCTCAGCTGAAATACACG GGCTTGGATTATGGCTGTCTGTCGG---CGT--AAAGTC--GGCTCAGCTGAAATACACG GGCTTGGATTATGGCTG-CTGCCGG----CTTTCATTAGTCGACTCGGCTGAAATACACG GGCTTGGATTATGGCTG-CTGCCGG----CTTTCGTTAGTCGACTCGGCTGAAATACACG GGCTTGGATATCGCTG-CTCCCGG--OCTTTCGTTAGTCGACTCGGCTCARATACACG

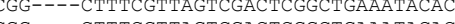
GGCTTGGATTATGGCTG-CTGCCGG----CTTTCATTAGTCGACTCGGCTGAAATACACG GGCTTGGATTATGGCTG-CTGCCGG----CTTTCATTAGTCGACTCGGCTGAAATACAC

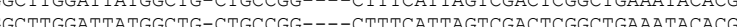
GGCTTGGATTATGGCTG-CTGCCGG----CTTTCATTAGTCGACTCGGCTGAAATACACG GGCTTGGATTATGGCTG-CTGTCGG---CGT--AATTGCCGG-CTCAGCTGAAATACACG GGCTTGGATTATGGCTG-CTGTCGG---CGT--AATTGCCGG-CTCAGCTGAAATACACG GGCTTGGATTATGGTTGT-TGTCGG---CGT--AATTGCCGG-CTCAACTGAAATACACG GGCTTGGATTATGGTTGT-TGTCGG---CGT--AATTGCCGG-CTCAACTGAAATACACG GGCTTGGATTATGGTTGT-TGTCGG---CGT--AATTGCCGG-CTCAACTGAAATACACG GCTTCG

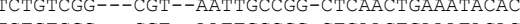
GGCTTGGATTATGGTTGTCTGTCGG---CGT--AATTGCCGG-CTCAACTGAAATACACG GGCTTGGATTATGGTTGTCTGTTGG---CGT--AATTGCCGG-CTCAACTGAAATACACG GGCTTGGATTATGGTTGTCTGTTGG---CGT--AATTGCCGG-CTCAACTGAAATACACG GGCTTGGATTATGGTTGTCTGTTGG---CGT--AATTGCCGG-CTCAACTGAAATACACG GGCTTGGATTATGGTTGTCTGTTGG---CGT--AATTGCCGG-CTCAACTGAAATACACG GGCTTGGATTATGGTTGTCTGTCGG---CGT--AATTGCCGG-CTCAACTGAAATACACG GGCTTGGATTATGGTTGTCTGTCGG---CGT--AATTGCCGG-CTCAACTGAAATACACG GGCTTGGATTATGGTTGTCTGTCGG---CGT--AATTGCCGG-CTCAACTGAAATACACG GGCTTGGATTATGGTTGTCTGTCGG---CGT--AATTGCCGG-CTCAACTGAAATACACG GGATCGA GACTCG

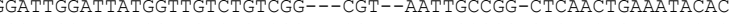
GCCITGGATTATGGT TGTCGGTCG--CGT--AATTGCCGG-CICAACTGAAA GGCTTGGATTATGGTTGTCTGTCGG---CGT--AATTGCCGG-CTCAACTGAAATACACG 
C. chernovii FN400936

C. oeirensis AF444364 E5S-PDA
E3M-STD

Fungal sp. Gu213437

R. mucilaginosa AF444614 Q5S-2-PDA

R. mucilaginosa AF444609

R. mucilaginosa AF444503

R. mucilaginosa AF444503

R. mucilaginosa FR690147

R. mucilaginosa HQ702343

R. mucilaginosa FJ515212

R. mucilaginosa EU149812

R. mucilaginosa EU871492

Uncultured fungus FM875856

R. mucilaginosa CBS8618

R. mucilaginosa AF444635

K. malvinella AF444498

K. malvinella DQ667165

K. aeria AM922224

K. aeria AF444562

K. aeria AF444675

H10S-PDA

Basidiomycete BSU65613

R. sp. FJ238090

R. sp. FJ865356

R. oryzicola $A Y 335160$

R. SP. FJ008055

S. dacryoidea AF444571 Z4S-1-PDA

S. dacryoidea AF444597

S. dacryoidea AF444500

S. dacryoidea AB049027

S. dacryoidea DQ832207

R. minuta AB078490

R. minuta $A B 078484$

R. minuta $A B 038117$ B 4M-STD

R. minuta $A B 078501$

R. minuta AF190010

R. minuta AF190010

R. minuta AB078497 I1-STD

R. sp. AF444619

R. Slooffiae FJ515215

R. slooffiae AB025994 J3S-STD

R. Slooffiae FJ807685

R. Slooffiae AF444589

R. slooffiae AB078491

R. Slooffiae AB052212

R. Sloolliae AF444627

R. Slooffiae AF444552

R. Slooffiae AB078495
R. slooffiae CBS8411
AGCTAGATCTGTCTC----0-----TATGACTGGTTTGA--CTT---GGCAT---GCTTCCATCTGTCT---_-_-_-_-ATATGACTGGTTTGA--CTT---GGCA----_ AGCTGGATCTGTCT----------ATATGACTGGTTTGA--CTT---GGCA---AGC---ATCCGCAATC-G---------AACTT--CGGATTGA--CTT---GGCG-----T AGC---ATCCGCAATC-G---------AACTT--CGGATTGA--CTT---GGCG-----T AGC---ATCCGCAATC-G---------AACTT--CGGATTGA--CTT---GGCG-----T AGC----ATCCGCAATC--------G---AACTT--CGGATTGA--CTT---GGCG-----T AGC---ATCCGCAATC--------G---AACTT--CGGATTGA--CTT---GGCG-----T (1)

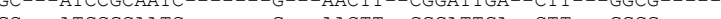

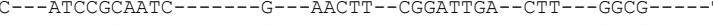
AGC---ATCCGCAATC--------G---AACTT--CGGATTGA--CTT---GGCG-----T AGC---ATCCGCAATC--------G---AACTT--CGGATTGA--CTT---GGCG-----T AGC---ATCCGCAATC--------G---AACTT--CGGATTGA--CTT---GGCG-----T AGC---ATCCGCAATC--------G---AACTT--CGGATTGA--CTT---GGCG-----T AGC---ATCCGCAATC--------G---AACTT--CGGATTGA--CTT---GGCG-----T AGC---ATCCGCAATC--------G---AACTT--CGGATTGA--CTT---GGCG-----T AGC---ATCCGCAATC--------G---AACTT--CGGATTGA--CTT---_GGCG-----T AAGTTGAATGGTT--0---A--0--CATTTGCACCTTGAC-CT----GATG---AGCAAGTTGAATGGTTTCC----1-0---OTTTCAGCTTGA--CCTGATGTTGTAAACT AAGTTGAATGGTTTCC------------ATTTGCAGCTTGAC-CTG----ATG-----T AGTTGAATGGTTTCC----A---------TTTGCAGCTTGAC-CT----GATG-----T AGTTGAATGGTTTCC------------ATTTGCAGCTTGAC-CTG----ATG-----T AGC---AACCCATTT----------GAAATAGACGGTTTGACTC-----GGCGT---AGC---AACCCATTT--------G---AAATAGACGGTTTGACTC-----GGCG-----T AGC---AACCCATTT--------G---AAATAGACGGTTTGACTC-----GGCG-----T AGC---AACCCATTT--------G---AAATAGACGGTTTGACTC-----GGCG-----T AGCTGCCCCGGTGTTCTCT-TTCGAAGAAACAAACGGCTTGACTC-----GGCGT----AGCT---ACCCCGATTTCTTTCTTAAGAAATAGACGGTTCGACTC-----GGCG-----

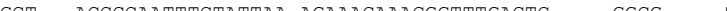
(1)

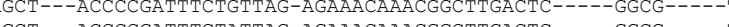
AGCT--ACCCCGATTICIATTAG-AGAAACAAACGGCITGACTC----GGCG---AGCT--ACCCCGATITCTATTAG-AGAAACAAACGGCITGACTC-----GGCG----CTC-----GGCG----AGCT---ACCCCGATTTCTATTAG-AGAAACAAACGGCTTGACTC------GGCG-----T AGC---AACCCATTT--------_---AAATAAACGGCTTGACTC-----GGCG-----T AGC---AACCCATTT--------G---AAATAAACGGCTTGACTC-----GGCG-----T AGC---AACCCCTTT--------G---AAATAGACGGTTTGACTC-----GGCG-----T AGC---AACCCCTTT----------GAAATAGACGGTTTGACTC-----GGCGT----AGC---AACCCCTTT--------G---AAATAGACGGTTTGACTC-----GGCG-----T AGC-ACCAGC--AACCCTATT---

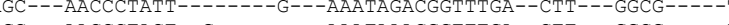
AGC---AACCCTACT--G---1---AAATAAACGGTTTGA--CTT---GGCG-----T AGC--AACCCTACT-------G--AAATAAACGGIITGA-CIT--GGCG---AGC---AACCCTACT--------G---AAACAAACGGTTTGA--CTT---GGCG-----T AGC---AACCCTACT--------G---AAATAAACGGTTTGA--CTT---GGCG-----T AGC---AACCCTATT--G--------AAATAAACGGTTTGA--CTT---GGCG-----T AGC---AACCCTATT--------G---AAATAAACGGTTTGA--CTT---GGCG-----T AGC---AACCCTATT--------G---AAATAAACGGTTTGA--CTT---GGCG-----T AGC----AACCCTATT---------G---AAATAAACGGTTTGA--CTT---_GGCG-----T AGC---AACCCTATT------G-- AAATAAACGGTTTGA--CTT---GGCG--CCAGC-AACCCIATH--AGCAGC---AACCCTATT--------G---AAATAAACGGTTTGA--CTT---GGCG-----T 
C. chernovii FN400936 C. magnus AF190009 C. oeirensis AF444364 E5S-PDA
E3M-STD

Fungal sp. GU213437

R. mucilaginosa AF444614 Q5S-2-PDA

R. mucilaginosa AF444609 R. mucilaginosa AF444503 R. mucilaginosa AF444503
R. mucilaginosa CBS2382

R. mucilaginosa FR690147

R. mucilaginosa HQ702343

R. mucilaginosa FJ515212

R. mucilaginosa EU149812

R. mucilaginosa EU871492 Uncultured fungus FM875856

R. mucilaginosa CBS8618 R. mucilaginosa AF444635 K. malvinella AF444498 K. malvinella AF444498 K. aeria AM922224 K. aeria AM922224
K. aeria AF444562 K. aeria AF444562
K. aeria AF444675 H1OS-PDA

Basidiomycete BSU6561 R. sp. FJ238090

R. sp. FJ865356 P2S-PDA

R. oryzicola AY335160 R. sp. FJ008055

S. dacryoidea AF444571 Z4S-1-PDA

S. dacryoidea AF444597

S. dacryoidea AF444500

S. dacryoidea AB049027

S. dacryoidea DQ832207

R. minuta AB078490

R. minuta AB078484

R. minuta AB038117 B 4M-STD

R. minuta $A B 078501$

R. minuta AF190010

R. minuta AF190010

R. minuta AB078493
R. minuta AB078497 I1-STD

R. sp. AF444619

R. Slooffiae FJ515215

R. slooffiae AB025994 J $3 S-S T D$

R. Slooffiae FJ807685

R. Slooffiae AF444589

R. slooffiae AB078491

R. slooffiae AB052212

R. sloofiae AB052212

R. Sloolfiae AF444627

R. Slooffiae AF444552

R. Slooffiae AB078495
R. slooffiae CBS8411

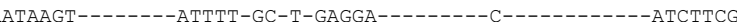
ATAAGT--------ATTTT-GC-T-GAGGA--------C-------------ATCTTCG AATAAGT--------ATTTT-GC-T-GAGGA-----------------CATCTTCG AATAGACT-------ATTC--GC-T-GAGGA-------------ATT--CTAGTCTTCG AATAGACT-------ATTC--GC-T-GAGGA-------------ATT--CTAGTCTTCG AATAGACT-------ATTC--GC-T-GAGGA------------ATT--CTAGTCTTCG AATAGACT-------ATTC--GC-T-GAGGA-------A------TT--CTAGTCTTCG AATAGACT-------ATTC--GC-T-GAGGA-------A-------TT--CTAGTCTTCG

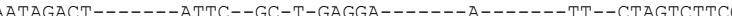
A A TATCACT--ATTC--GC-T-GAGGA-------A------TT--CTAGTCTTCG AATAGACT-------ATTC--GC-T-GAGGA-------A-------TT--CTAGTCTTCG AATAGACT-------ATTC--GC-T-GAGGA-------A-------TT--CTAGTCTTCG AATAGACT-------ATTC--GC-T-GAGGA-------A-------TT--CTAGTCTTCG AATAGACT------ATTC--GC-T-GAGGAA---------T-----TCTAGTCTTCG AATAGACT-------ATTC--GC-T-GAGGA-------A-------TT--CTAATCTTCG AATAGACT-------ATTC--GC-T-GAGGA-------A-------TT--CTAATCTTCG TGTAAACT--------ACT----CATCGGGGA---T------------G--CAATGC---

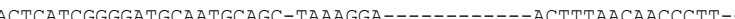
CTCATCGCGATTGCATGGAGC-TAAAGGAACTTTAACAG--_-_-_-_-_-_-_-_-

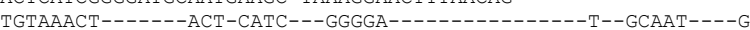
TGTAAACT-------ACT----CATCGGGGA---------------T--GCAAT----GTAAACT-------ACT-CATC---GGGGA---------------T--GCAAT---AATAATT-------ATTTC-GC-T-GAGGA--------C--------GCTTCTTAATAATT-------ATTTC-GC-T-GAGGAC---------G-------TCTTCTT-AATAATT-------ATTTC-GC-T-GAGGAC---------G-------TCTTCTT-AATAATT-------ATTTC-GC-T-GAGGA----------------CGTCTTCT AATAACAT-------ATTTC-GC-T-GAGGAC-------G------TT--ACATTCTTAT ААТАACT--------ATTTC-GC-T-GAGGAC----------G-------TCACATTC ATABCAT--0-TACATA

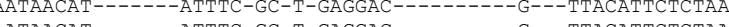
MATAACAT-AATAACAT-1- -ATTIC-GC-I-GAGGAC---------G---TIACATICICTAAG ATTCTCTAAG AATAACAT-------ATTTC-GC-T-GAGGAC----------G---TTACATTCTCTAAG AATAATT--------ATTTC-GC-T-GAGGAC------G-----------TTTTCTTCA AATAATT-------ATTTC-GC-T-GAGGAC------G----------TTTTCTTCA AАTAATT------ATTTC-GC-T-GAGGAC------G---------TCTTCTTCA

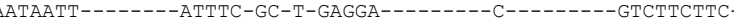
AАTAATT--------ATTTC-GC-T-GAGGAC------G-----------TCTTCTTC AATAATT--0-A AATATCHATAATI--AATAATT-----ATTTC-GC-I-GAGGA-----------C--GTTTTCTTCA AATAATTAATAATT-------ATTTC-GC-T-AAGGAC---------G------TTTTCTTCA AATAATT--------ATTTC-GC-T-AAGGAC---------G-------TTTTCTTCA AATAATT-------ATTTC-GC-T-AAGGA-------------C--GTTTTCTTCAATAATT-------ATTTC-GC-T-AAGGAC---------G-------TTTTCTTCAATAATT-------ATTTC-GC-T-AAGGAC---------G-------TTTTCTTCAАTAATT--------ATTTC-GC-T-AAGGAC----------G--------TTTTCTTC

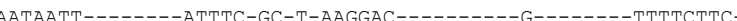
A TAC AATAATT-------ATTIC-GC-T-AAGGAC--------_G------CTTTCTTC 
C. chernovii FN400936 C. magnus AF19000

C. oeirensis $\mathrm{AF} 444364$ E5S-PDA
E3M-STD

Fungal sp. GU213437

R. mucilaginosa AF444614 $5 S-2-P D A$

R. mucilaginosa AF444609

R. mucilaginosa AF444503

R. mucilaginosa AF444503

R. mucilaginosa FR690147

R. mucilaginosa HQ702343

R. mucilaginosa FJ515212

R. mucilaginosa EU149812

R. mucilaginosa EU871492

Uncultured fungus FM875856

R. mucilaginosa CBS8618

R. mucilaginosa AF444635

K. malvinella AF444498

K. malvinella DQ667165

K. aeria AM922224

K. aeria AF444562

K. aeria AF444675 H10S-PDA

Basidiomycete BSU65613

R. sp. FJ238090

R. sp. FJ865356

R. oryzicola $A Y 335160$

R.

S. dacryoidea AF444571 Z4S-1-PDA

S. dacryoidea AF444597

S. dacryoidea AF444500

S. dacryoidea AB049027

S. dacryoidea DQ832207

R. minuta AB078490

R. minuta AB078484

R. minuta $A B 038117$ B 4M-STD

R. minuta $A B 078501$

R. minuta AF190010

R. minuta AF 190010

R. minuta AB078497 I1-STD

R. sp. AF444619

R. Slooffiae FJ515215

R. Slooffiae AB025994 J3S-STD

R. Slooffiae FJ807685

R. Slooffiae AF444589

R. Slooffiae AB078491

R. Sloofiae AB052212

R. Sloolfiae AF444627

R. Slooffiae AF444552

R. Slooffiae AB078495
AT---GGCC-_-_-_-_-_-_-AGGACCTTGACTATTGTCTGCTAACTAAACCATTAC ---ATGGCC------------AGGACCTAGACTACTGTCTGCTAACTAAACCATCA G---ATGGCC----------AGGACCTAGACTATTGTCTGCTAACTAAACCATCAC GATTAGAGCC-----------GGGTTG--GGTTAAAGGAAGTT------TC----GACTAGAGCC-------------GGGTTG--GGTTAAAGGAAGCT------TC-----

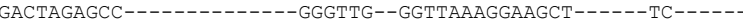
GACTAGAGCC--------G--------GGTTG--GGTTAAAGGAAGCT------TC-T---GACTAGAGCC--------G-------GGTTG--GGTTAAAGGAAGCT------TC-T---

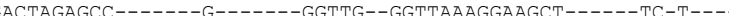

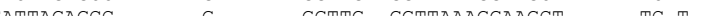
GATTACACCGACIAGAGCC-1GACIAGAGCC--GACTAGAGCC------G---1--GGTTG--GGITAAAGGAAGCT------IC-T--GACTAGAGCC------G------GGTTG--GGTTAAAGGAAGCT------TC-T--GATTAGAGCC-------G------GGTTG--GGTTAAAGGAAGCT------TC-T--GATTAGAGCC-------G-------GGTTG--GGTTAAAGGAAGCT------TC-T--GACTAGAGCC-------------GGGTTG--GGTTAAAGGAAGCT------TC-----GATTAGAGCC--------G-------GGTTG--GGTTAAAGGAAGCT------TC-T---GATTAGAGCC-------G-------GGTTG--GGTTAAAGGAAGCT------TC-T----

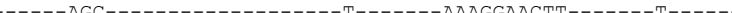

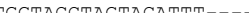

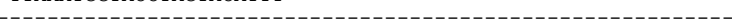

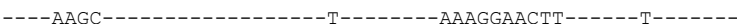

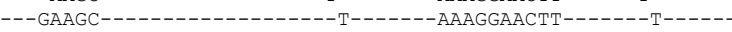
(1)

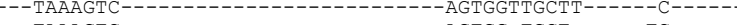
- TAAAGTC---- TAAAGTC----

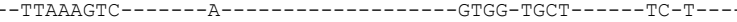
GTTAAGATTA--------C------------AATGGTGG-TGCT------TC--T-TAATACACAC-----------A-----AAGAATCTTAGTGG-TGCT------TC----

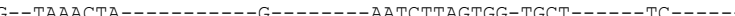
TAA- $A$ A TAR-AAAGAATATAAAGAA-TIATAAGAA--10 TATAAAGA---0---0------

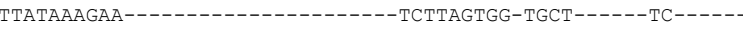

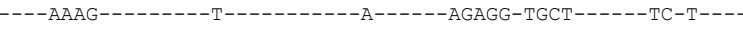

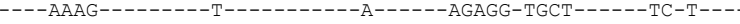

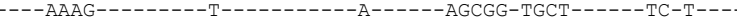
- AAAAGT---

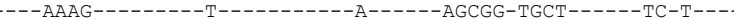
--

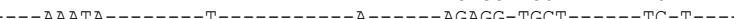
ATTATA-A-10

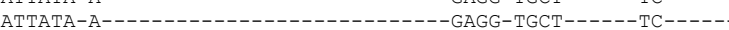
-ATTATA-A-----_--

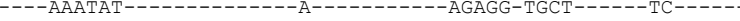

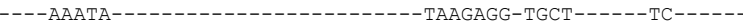
- AAATA---_-

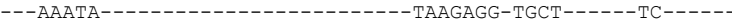
---AAATA-----------------------TAAGAGG-TGCT------TC-----

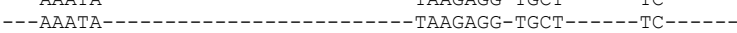


C. chernovii FN400936 C. magnus AF190009 C. oeirensis AF444364 E5S-PDA
E3M-STD

Fungal sp. GU213437

R. mucilaginosa AF444614 Q5S-2-PDA

R. mucilaginosa AF444609 R. mucilaginosa AF444503

R. mucilaginosa AF444503

R. mucilaginosa FR690147

R. mucilaginosa HQ702343

R. mucilaginosa FJ515212

R. mucilaginosa EU149812

R. mucilaginosa EU871492 Uncultured fungus FM875856

R. mucilaginosa CBS8618 R. mucilaginosa AF444635 K. malvinella AF444498 K. aeria AM922224 K. aeria AM922224
K. aeria AF444562 K. aeria AF444562
K. aeria AF444675 H10S-PDA

Basidiomycete BSU65613 R. sp. FJ238090

R. sp. FJ865356 P2S-PDA

R. oryzicola AY335160 R. sp. FJ008055

S. dacryoidea AF444571 Z4S-1-PDA

S. dacryoidea AF444597

S. dacryoidea AF444500

S. dacryoidea AB049027

. dacryoidea DQ832207

R. minuta AB078490

R. minuta $A B 078484$

R. minuta $A B 038117$ B4M-STD

R. minuta $A B 078501$

R. minuta AF190010

R. minuta AF 190010

R. minuta AB078497 I1-STD

R. $\mathrm{sp}$. AF444619

R. Slooffiae FJ515215

R. Slooffiae AB025994 J3S-STD

R. Slooffiae FJ807685

R. Slooffiae AF444589

R. Slooffiae AB078491

R. sloofiae AB052212

R. Slooffiae AF444627

R. Slooffiae AF444552

R. Slooffiae AB078495
R. slooffiae CBS8411
TTTAA---GTGCATCTTTGGATGTTACTCATTGTA---TA-ACTTTGA-CATCTGGCCTC TITATTTAA---GTGCATCTTTGGATGTTACTAATTGTG---TA-ACTTTGA-CATCTGGCCTC --TAAT--CAGAATGTCT---------ACATTTTA---AG----ATTA-GA-----TCTC --TAAT--CAGAATGTCT---------ACATTTTA---AG----ATTA-GA-----TCTC --TAAT--CAGAATGTCT---------ACATTTTA---AG----ATTA-GA-----TCTC ---AAT--CAGAATGTCT--A-------CATTTTA---AG----ATTA-GA-----TCTC ---AAT--CAGAATGTCT--A-------CATTTTA---AG----ATTA-GA-----TCTC --AAT--CAGAATGTCT--A------_CATTTTA---AG----ATTA-GA-----TCT --AAT--CAGAATGTCT--A-------CATTTTA---AG----ATTA-GA-----TCTC --AAT--CAGAATGTCT--A-------CATTTTA---AG----ATTA-GA-----TCTC ---AAT--CAGAATGTCT--A-------CATTTTA---AG----ATTA-GA-----TCTC --TAATC--AGAATGTCT---------ACATTTTA---AG----ATTA-GA-----TCTC

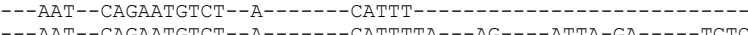

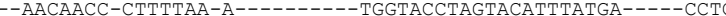

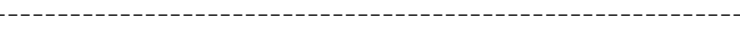
--AA---CAGAACCCCT--------AAATGGTACCTAGTACATTTATGA-----CCTC --AA---CAGAACCCCTTA--------AATGGTACCTAGTACATTTATGA-----CCTC --AA---CAGAACCCCTTGAATGGTACCTAGTAC---A-----TTTATGA-----CCTC --TAAT----GCGTATTATA--------CATCATA---AG-CCTTTGA-GAC----CCTC --TAAT----GCGTATCAT-------ACACTTTT---AG---CTTTA-GA-----CCTC --TAAT----GCGTATCAT-------ACACTTTT---AG---CTTTA-GA-----CCTC ---AAT----GCGTATCAT-A-------CACTTTT---AG---CTTTA-GA-----CCTC ---AAT----GCGTCTGTTCAG-TCAGACACCTTA---AGC---TTTA-GA-----CCTC -TAAT----GCGTCTGTTTAGTCAGACAACCTTA---AG---CTTTA-GA-----CCTC -TAAT--OCCTCTGTTCA-GTCAGACACCTTA--AG--CTMTA-CA----CCTC -TAAT----GCGTCTGITCA-GICAGACACCITA--AG---CTITA-GA----CCTC -TAAT---GCGTCTGTTCA-GICAGACACCTTA---AG---CTTTA-GA-----CCTC -TAAT--GCGTCTGTICA-GTCAGACACCITA--AG--CITIA-GA-------TAAT----GCGTCTGTTCA-GTCAGACACCTTA---AG---CTT-

---AAT--GCGCTTTTAA--A------GCAACTTA---AG---CTTTA-GA-----CCTC ---AAT--GCGCTTTTAA--A------GCAACTTA---AG---CTTTA-GA-----CCTC ---AAT--GCGCTTTTAA--A------GCATCTTA---AG---CTTTA-GA-----CCTC --TAAT--GCGCTTTTAA-A-------GCATCTTA---AG-C--TTTA-GA-----CCTC ---AАT--GCGCTTTTAA--A------GCATCTTA---AG---СTTTA-GA-----CСТС --AAT--GCGCTT-TAT--A--TAAT--TCGCTTCTAAT-----AGCAT-TTA---AG-C--TTTA-GA----CCTC -TAATI--CGCITCTAAT-----AGCAT-TIA--AG---CTTTA-GA-----CCTC -TAATT--CGCTTCTAAT-------AGCAT-TTA---AG---CTTTA-GA-----CCTC --TAATT--CGCTTCTAAT-------AGCAT-TTA---AG---CTTTA-GA-----CCTC --TAAT--TCGCTTCTAAT------AGCAT-TTA---AG-C--TTTA-GA-----CCTC --TAATT--CGCTTCTAAT------AGCAT-TTA---AG---CTTTA-GA-----CCTC --TAATT--CGCTTCTAAT-------AGCAT-TTA---AG---CTTTA-GA-----CCTC -TAATT--CGCTTCTAAT------AGCAT-TTA---AG---CTTTA-GA-----CCTC -TAATT--CGСТTCTAAT---0-AGCAT-TTA---AG--CTTTA-GA----CCTC -TAAT-CCCTTCTAAT----O- TAC

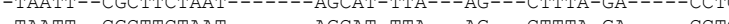
-TAATT--CGCTTCTAAT-------AGCAT-TTA---AG---CTTTA-GA-----CCTC 
C. chernovii FN400936 C. magnus AF190009 C. oeirensis AF444364 E5S-PDA

Fungal sp. GU213437

R. mucilaginosa AF444614 25S-2-PDA

R. mucilaginosa AF444609

R. mucilaginosa AF444503

R. mucilaginosa CBS2382

R. mucilaginosa FR690147

R. mucilaginosa HQ702343

R. mucilaginosa FJ515212

R. mucilaginosa EU149812

R. mucilaginosa EU871492

Uncultured fungus FM875856

R. mucilaginosa CBS8618

R. mucilaginosa AF444635

K. malvinella AF444498

K. malvinella DQ667165

K. aeria AM922224

K. aeria AF444562

K. aeria AF444675

H10S-PDA

Basidiomycete BSU65613

R. sp. FJ238090

R. sp. FJ865356

R. oryzicola AY335160

R. sp. FJ008055

S. dacryoidea AF444571 Z4S-1-PDA

S. dacryoidea AF444597

S. dacryoidea AF444500

S. dacryoidea AB049027

S. dacryoidea DQ832207

R. minuta AB078490

R. minuta AB078484

R. minuta AB038117 B 4M-STD

R. minuta $\mathrm{AB} 078501$

R. minuta AF190010

R. minuta AF190010

R. minuta AB078493
R. minuta AB078497 I1-STD

R. $\mathrm{sp}$. AF444619

R. Slooffiae FJ51521

R. slooffiae AB025994 J3S-STD

R. Slooffiae FJ 807685

R. Slooffiae AF444589

R. Slooffiae AB078491

R. Slooffiae AB052212

R. slooffiae AF444627

R. Slooffiae AF44462

R. Slooffiae AF444552

R. Slooffiae AB078495
R. slooffiae CBS8411
AAATCAGGTAGGACTACCCGCTGAACTTAAGCA AATCAAGTAGGACTACCCGCTGAACTTAAGCA AAATCAGGTAGGACTACCCGCTGAACTTAAGCA AAATCAGGTAGGACTACCCGCTGAACTTAA--AAATCAGGTAGGACTACCCGCTGAACTTAA--AAATCAGGTAGGACTACCCGCTGAACTTAAGCA AAATCAGGTAGGACTACCCGCTGAACTTAAGCA AAATCAGGTAGGACTACCCGCTGAACTTAA--AAATCAGGTAGGACTACCCGCTGAACTTAAGCA AATCACGTAGGACTACCCGCTGAACTTAA AARTCATAGGACTACCCGCTGAACITAAGCA

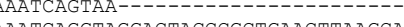
AAATCAGGTAGGACTACCCGCTGAACTTAAGCA AAATCAGGTAGGACTACCCGCTGAACTTAAGCA AAATCAGGTAGGACTACCCGCTGAACTTAAGCA AAATCAGGTAGGACTACCCGCTGAACTTAA--AAATCAGGTAGGACTACCCGCTGAACTTAAGCA AAATCAGGTAGGACTACCCGCTGAACTTAAGCA AATCAAGTAGGACTACCCGCTGAACTTAAGCA AATCAAGTAGGACTACCCGCTGAACTTAAGCA AAATCAAGTAGGACTACCCGCTGAACTTAA--AAATCGGTCAGGATTACCCGCTGAACTT---AAATCAGGCAGGACTACCCGCTGAACTTAAGCA AAATCAGGCAGGACTACCCGCTGAACTTAA--AAATCAGGCAGGACTACCCGCTG---_-_-_AAATCAGTCAGGACTACCCGCTG---------AAATCAGTCAGGACTACCCGCTGAACTTAAGCA AAATCAGTCAGGACTACCCGCTGAACTTAAGCA

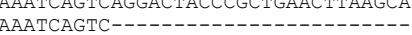
AAATCAGTC-1AAATCAGTCAGGACTACCCGCTGAACTTAAGCA

-

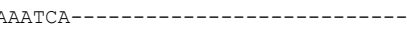

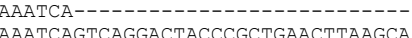
AAATCAGTCAGGACTACCCGCTGAACTTAA--AAATCAGTCAGGACTACCCGCTGAACTTAAGCA AATCACTCAGCACTACCCGCTGACTTAAGCA AAACAGGCA

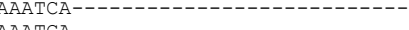
AATCAAAATCAGTCAGGACTACCCGCTGAACTTAA--AAATCAGTCAGGGCTACCCGCTGAACTTAAGCA AAATCAGTCAGGACTACCCGCTGAACTTAAGCA AAATCAGTCAGGACTACCCGCTGAACTTAAGCA AAATCAGTCAGGACTACCCGCTGAACTTAA--AAATCAGTCAGGACTACCCGCTGAACTTAAGCA AAATCAGTCAGGACTACCCGCTGAACTTAAGCA

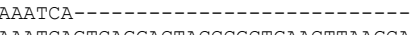
AAATCAGTCAGGACTACCCGCTGAACTTAAGCA AAATCAGTCAGGACTACCCGCTGAACTTAAGCA AAATCAGTCAGGACTACCCGCTGAACTTAAGCA

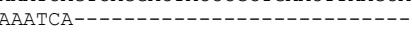




\section{References}

Abe, F., T. Miura, et al. (2001). "Isolation of a highly copper-tolerant yeast, Cryptococcus sp., from the Japan Trench and the induction of superoxide dismutase activity by $\mathrm{Cu} 2+. "$ Biotechnology Letters (23): 2027-2034.

Ahearn, D. G. and S. P. Meyers (1976). Fungal Degradation of Oil in the Marine Environment. Recent advances in aquatic mycology. G. E. B. Jones. New York, Halsted Press.

Ahearn, D. G., F. J. Roth, Jr., et al. (1962). "A comparative study of marine and terrestrial strains of Rhodotorula." Canadian Journal of Microbiology 8: 121-132.

Ahearn, D. G., F. J. Roth, Jr., et al. (1968). "Ecology and characterization of yeasts from aquatic regions of South Florida." Marine Biology 1(291-308).

Al Rwahnih, M., S. Daubert, et al. (2011). "Deep sequencing evidence from single grapevine plants reveals a virome dominated by mycoviruses." Archives of virology 156(3): 397-403.

Altschul, S. F., W. Gish, et al. (1990). "Basic local alignment search tool." Journal of Molecular Biology 215(3): 403-410.

Arenz, B., B. Held, et al. (2006). "Fungal diversity in soils and historic wood from the Ross Sea Region of Antarctica." Soil Biology and Biochemistry 38(10): 3057-3064.

Baas Becking, L. G. M. (1934). Geobiologie of Inleiding tot de Milieukunde. The Hague, W.P. Van Stockum \& Zoon.

Bass, D., A. Howe, et al. (2007). "Yeast forms dominate fungal diversity in the deep oceans." Proceedings of the Royal Society B: Biological Sciences 274(1629): 3069-3077.

Benson, D. A., I. Karsch-Mizrachi, et al. (2005). "GenBank." Nucleic acids research 33(Database issue): D34-38.

Boenigk, J., K. Pfandl, et al. (2006). "Evidence for geographic isolation and signs of endemism within a protistan morphospecies." Applied and Environmental Microbiology 72(8): 5159-5164.

Boo, S. M., H. S. Kim, et al. (2010). "Complex phylogeographic patterns in the freshwater alga Synura provide new insights into ubiquity vs. endemism in microbial eukaryotes." Molecular Ecology 19(19): 4328-4338.

Botstein, D., S. A. Chervitz, et al. (1997). "Yeast as a model organism." Science 277(5330): 1259-1260. 
Boundy-Mills, K. (2006). Methods for Investigating Yeast Biodiversity. Biodiversity and Ecophysiology of Yeasts. C. Rosa and G. Pétér. Germany, Springer-Verlag. 1: 67-100.

Brown, M. R., S. M. Barrett, et al. (1996). "Biochemical composition of new yeasts and bacteria evaluated as food for bivalve aquaculture." Aquaculture 143: 341-360.

CBS-KNAW (2012). "CBS-KNAW fungal biodiversity centre." Retrieved 01/01/2012, from http://www.cbs.knaw.nl/.

Chang, C. (2000). "Immunomodulation by dietary $\beta$-1, 3-glucan in the brooders of the black tiger shrimp Penaeus monodon." Fish \& Shellfish Immunology 10(6): 505-514.

Chi, Z.-M., G. Liu, et al. (2010). "Marine yeasts as biocontrol agents and producers of bioproducts." Applied Microbiology and Biotechnology 86(5): 1227-1241.

Chi, Z., Z. Chi, et al. (2009). "Production, characterization and gene cloning of the extracellular enzymes from the marine-derived yeasts and their potential applications." Biotechnology Advances 27(3): 236-255.

Chi, Z., Z. Chi, et al. (2009). "Inulinase-expressing microorganisms and applications of inulinases." Appl Microbiol Biotechnol 82: 211-220.

Chi, Z., Z. Liu, et al. (2006). "Marine yeasts and their applications in mariculture." Journal of Ocean University of China 5(3): 251-256.

Chi, Z., F. Wang, et al. (2009). "Bioproducts from Aureobasidium pullulans, a biotechnologically important yeast." Applied Microbiology and Biotechnology 82(5): 793-804.

Chi, Z., F. Wang, et al. (2007). "Selection of Yarrowia lipolytica strains with high protein content from yeasts isolated from different marine environments." Journal of Ocean University of China 6(4): 360-364.

Chi, Z., K. Yan, et al. (2008). "Diversity of marine yeasts with high protein content and evaluation of their nutritive compositions." Journal of the Marine Biological Association of the United Kingdom 88(07): 1347.

Da Costa, E. and J. D'Souza (1979). "Studies on estuarine yeasts: III. hydrocarbon degraders." Mahasagar-Bulletin of the National Institute of Oceanography 12(3): 155-161.

De Wit, R. and T. Bouvier (2006). "'Everything is everywhere, but, the environment selects'; what did Baas Becking and Beijerinck really say?" Environmental Microbiology 8(4): 755-758.

Deák, T. (1999). "Molecular taxonomy of yeasts." Acta Microbiologica et Immunologica Hungarica 46(23): 181-186. 
Deák, T. (2006). Environmental Factors Influencing Yeasts. Biodiversity and Ecophysiology of Yeasts. C. Rosa and G. Pétér. Germany, Springer-Verlag. 1: 155-174.

Demain, A. L., H. J. Phaff, et al. (1998). The Industrial and Agricultural Significance of Yeasts. The Yeasts, a taxonomic study. C. P. Kurtzman and J. W. Fell. Netherlands, Elsevier: 13-19.

Di Menna, M. E. (1955). "A search for pathogenic species of yeasts in New Zealand soils." Journal of General Microbiology 12: 54-62.

Dix, N. J. and J. Webster (1995). Fungal Ecology. Cambridge, Chapman \& Hall.

Dobell, C. (1932). Antony van Leeuwenhoek and His "Little Animals". New York, Harcoukt, Brace and Company.

Drummond, A. J., B. Ashton, et al. (2011). Geneious.

Fedorak, P. M., K. M. Semple, et al. (1984). "Oil-degrading capabilities of yeasts and fungi isolated from coastal marine environments." Canadian Journal of Microbiology 30: 565-571.

Fell, J. W. (1976). Yeasts in Oceanic Regions. Recent advances in aquatic mycology. G. E. B. Jones. New York, Halsted Press.

Fell, J. W. (2001). Collection and Identification of Marine Yeasts. Methods in Microbiology. J. H. Paul. Wiltshire, Academic Press. 30: 347-356.

Fell, J. W., D. G. Ahearn, et al. (1960). "Isolation of yeasts from Biscayne Bay, Florida and adjacent benthic areas." Limnology and Oceanography 5(4): 366-371.

Fell, J. W., T. Boekhout, et al. (2000). "Biodiversity and systematics of basidiomycetous yeasts as determined by large-subunit rDNA D1/D2 domain sequence analysis." International Journal of Systematic and Evolutionary Microbiology 50: 1351-1371.

Fell, J. W. and I. L. Hunter (1968). "Isolation of heterothallic yeast strains of Metschnikowia kamienski and their mating reactions with Chlamydozyma wickerham spp." Antonie van Leeuwenhoek 34: 365-376.

Fell, J. W. and C. P. Kurtzman (1990). "Nucleotide sequence analysis of a variable region of the large subunit rRNA for identification of marine-occurring yeasts." Current Microbiology 21: 295300 .

Fell, J. W. and J. I. Pitt (1969). "Taxonomy of the yeast genus Metschnikowia: a correction and a new variety." Journal of Bacteriology 98(2): 853-854.

Fell, J. W. and N. Van Uden (1963). Yeasts in Marine Environments. Marine Microbiology. C. H. Oppenheimer. Springfield Illinois, Thomas, Charles C.: 329-340. 
Fenchel, T. and B. J. Finlay (2004). "The ubiquity of small species: patterns of local and global diversity." BioScience 54(8): 777-784.

Finlay, B. J. (2002). "Global dispersal of free-living microbial eukaryote species." $\underline{\text { Science }}$ 296(5570): 1061-1063.

Finlay, B. J. and G. F. Esteban (2001). "Ubiquitous microbes and ecosystem function." Limnetica 20(1): 31-43.

Finlay, B. J. and T. Fenchel (2004). "Cosmopolitan metapopulations of free-living microbial eukaryotes." Protist 155(2): 237-244.

Finlay, B. J., S. C. Maberly, et al. (1997). "Microbial diversity and ecosystem function." Oikos 80(2): 209-213.

Fleet, G. H. (2001). "Biodiversity and ecology of Australasian yeasts (fungi)." Australian Systematic Botany 14: 501-511.

Gadanho, M. r., J. o. M. G. C. F. Almeida, et al. (2003). "Assessment of yeast diversity in a marine environment in the south of portugal by microsatellite-primed PCR." Antonie van Leeuwenhoek 84: 217-227.

Gao, L., Z. Chi, et al. (2007). "Inulinase-producing marine yeasts: evaluation of their diversity and inulin hydrolysis by their crude enzymes." Microbial Ecology 54(4): 722-729.

Gatesoupe, F. (2007). "Live yeasts in the gut: natural occurrence, dietary introduction, and their effects on fish health and development." Aquaculture 267(1-4): 20-30.

Gazis, R., S. Rehner, et al. (2011). "Species delimitation in fungal endophyte diversity studies and its implications in ecological and biogeographic inferences." Molecular Ecology 20(14): 3001-3013.

Giménez-Jurado, G., M. J. Valderrama, et al. (1995). "Assessment of phenotypic and genetic diversity in the yeast genus Metschnikowia." Antonie van Leeuwenhoek 68: 101-110.

Google Maps (2011). "Google Maps." Retrieved 01/01/2011, from http://maps.google.co.nz/maps?q=Wellington\&hl=en\&sll=$\underline{41.244772,172.617188 \& \mathrm{sspn}=17.731137,43.286133 \& \text { hnear }=W e l l i n g t o n \& \mathrm{t}=\mathrm{m} \& \mathrm{z}=10}$.

Hagler, A. N. and L. C. Mendonça-Hagler (1981). "Yeasts from marine and estuarine waters with different levels of pollution in the state of Rio de Janeiro, Brazil." Applied and Environmental Microbiology 41(1): 173-178.

Hasegawa, T., I. Banno, et al. (1960). "A taxonomic study on the genus Rhodotorula. I. The subgenus Rubrotorula nov. subgen " Journal of General and Applied Microbiology 5(4): 200-212. 
Hawksworth, D. L. (1998). Kingdom Fungi: Fungal Phylogeny and Systematics. Topley \& Wilson's Microbiology and Microbial Infections. L. Ajello and R. J. Hay. London, Arnold; Oxford University Press. 4.

Heath, R. A. (1985). "A review of the physical oceanography of the seas around New Zealand 1982." New Zealand Journal of Marine and Freshwater Research 19(1): 79-124.

Hernández-Montiel, L. G., J. L. Ochoa, et al. (2010). "Biocontrol of postharvest blue mold (Penicillium italicum Wehmer) on Mexican lime by marine and citrus Debaryomyces hansenii isolates." Postharvest Biology and Technology 56(2): 181-187.

Jain, M. R., S. S. Zinjarde, et al. (2004). "2,4,6-Trinitrotoluene transformation by a tropical marine yeast, NCIM 3589." Marine Pollution Bulletin 49(9-10): 783-788.

James, S. A., M. D. Collins, et al. (1996). "Use of an rRNA internal transcribed spacer region to distinguish phylogenetically closely related species of the genera Zygosaccharomyces and Torulaspora." International Journal of Systematic Bacteriology 46(1): 189-194.

Kang, C.-K., H. Y. Park, et al. (2006). "Use of marine yeasts as an available diet for mass cultures of Moina macrocopa." Aquaculture Research 37(12): 1227-1237.

Kohlmeyer, J. (1963). The Importance of Fungi in the Sea. Marine Microbiology. C. H. Oppenheimer. Springfield Illinois, Thomas, Charles C.: 300-314.

Kohlmeyer, J. and V. Demoulin (1981). "Parasitic and symbiotic fungi on marine algae." Botanica Marina 24(1): 9-18.

Kohlmeyer, J. and E. Kohlmeyer (1979). Marine Mycology The Higher Fungi. New York, Academic Press.

Kurtzman, C. and C. Robnett (2003). "Phylogenetic relationships among yeasts of the 'Saccharomyces complex' determined from multigene sequence analyses." FEMS Yeast Research 3(4): 417-432.

Kurtzman, C. P. (1994). "Molecular taxonomy of the yeasts." Yeast 10: 1727-1740.

Kurtzman, C. P. and P. A. Blanz (1998). Ribosomal RNA/DNA Sequence Comparisons for Assessing Phylogenetic Relationships. The Yeasts, a taxonomic study. C. P. Kurtzman and J. W. Fell. Netherlands, Elsevier: 69-74.

Kurtzman, C. P. and J. W. Fell (2006). Yeast Systematics and Phylogeny - Implications of Molecular Identification Methods for Studies in Ecology. Biodiversity and Ecophysiology of Yeasts. C. Rosa and G. Pétér. Germany, Springer-Verlag. 1: 11-30.

Kurtzman, C. P. and J. Piškur (2006). "Taxonomy and phylogenetic diversity among the yeasts." 15: $29-46$. 
Kurtzman, C. P. and C. J. Robnett (1998). "Identification and phylogeny of ascomycetous yeasts from analysis of nuclear large subunit (26S) ribosomal DNA partial sequences." Antonie van Leeuwenhoek 73: 331-371.

Kutty, S. N. and R. Philip (2008). "Marine yeasts—a review." Yeast 25(7): 465-483.

Lachance, M.-A., M. Miranda, et al. (1976). "Dehiscence and active spore release in pathogenic strains of the yeast Metschnikowia bicuspidata var. australis: possible predatory implication." Canadian Journal of Microbiology 22: 1756-1761.

Lachance, M. A. (2006). Yeast Biodiversity: How Many and How Much? Biodiversity and Ecophysiology of Yeasts. C. Rosa and G. Pétér. Germany, Springer-Verlag. 1: 1-9.

Legras, J.-L., D. Merdinoglu, et al. (2007). "Bread, beer and wine: Saccharomyces cerevisiae diversity reflects human history." Molecular Ecology 16(10): 2091-2102.

Lintott, E. A. and W. H. Lintott (2002). Marine Fungi of New Zealand. Fungi in Marine Environments. K. D. Hyde. Hong Kong, Fungal Diversity Press. 7: 285-292.

Lintott, W. H. and E. A. Lintott (1982). "Lignicolous marine fungi from New Zealand." Mauri Ora 10: 73-76.

MacGillivray, A. R. and M. P. Shiaris (1993). "Biotransformation of polycyclic aromatic hydrocarbons by yeasts isolated from coastal sediments." Applied and Environmental Microbiology 59(5): 1613-1618.

Magliani, W., S. Conti, et al. (1997). "Yeast killer systems." Clinical Microbiology Reviews 10(3): 369-400.

Martiny, J. B. H., B. J. M. Bohannan, et al. (2006). "Microbial biogeography: putting microorganisms on the map." Nature Reviews Microbiology 4(2): 102-112.

McKenzie, E. H. C. (2001). "Fungi anamorphici in Australasia." Australian Systematic Botany 14(485-500).

Mendonça-Hagler, L. C., A. N. Hagler, et al. (1993). "Phylogeny of Metschnikowia species estimated from partial rRNA sequences." International Journal of Systematic Bacteriology 43(2): 368-373.

Mendonça-Hagler, L. C., A. N. Hagler, et al. (1985). "DNA relatedness among aquatic yeasts of the genus Metschnikowia and proposal of the species Metschnikowia australis comb. nov." Canadian Journal of Microbiology 31: 905-909. 
Moore, M. M. and M. S. Strom (2003). "Infection and mortality by the yeast Metschnikowia bicuspidata var. bicuspidata in chinook salmon fed live adult brine shrimp (Artemia franciscana)." Aquaculture 220(1-4): 43-57.

MycoBank (2012). "International Mycological Association." Retrieved 01/01/2012, from http://www.mycobank.org/.

Nagahama, T. (2006). "Rhodotorula pacifica sp. nov., a novel yeast species from sediment collected on the deep-sea floor of the north-west Pacific Ocean." International Journal of Systematic and Evolutionary Microbiology 56(1): 295-299.

Nagahama, T. (2006). Yeast Biodiversity in Freshwater, Marine and Deep-Sea Environments. Biodiversity and Ecophysiology of Yeasts. C. Rosa and G. Pétér. Germany, Springer-Verlag. 1: 241-262.

Nagahama, T., M. Hamamoto, et al. (2001). "Distribution and identification of red yeasts in deepsea environments around the northwest Pacific Ocean." Antonie van Leeuwenhoek 80: 101-110.

Nagano, Y., T. Nagahama, et al. (2010). "Fungal diversity in deep-sea sediments - the presence of novel fungal groups." Fungal Ecology 3(4): 316-325.

Nerurkar, A. S., K. S. Hingurao, et al. (2009). "Bioemulsifiers from marine miroorganisms." Journal of Scientific \& Industrial Research 68: 273-277.

Nguyen, N. H., S.-O. Suh, et al. (2006). "Metschnikowia noctiluminum sp. nov., Metschnikowia corniflorae sp. nov., and Candida chrysomelidarum sp. nov., isolated from green lacewings and beetles." Mycological Research 110(3): 346-356.

Nilsson, R. H., E. Kristiansson, et al. (2008). "Intraspecific ITS variability in the kingdom Fungi as expressed in the international sequence databases and its implications for molecular species identification." Evolutionary Bioinformatics 4: 193-201.

O'Brien, H. E., J. L. Parrent, et al. (2005). "Fungal community analysis by large-scale sequencing of environmental samples." Applied and Environmental Microbiology 71(9): 5544-5550.

Oswal, N., P. M. Sarma, et al. (2002). "Palm oil mill effluent treatment by a tropical marine yeast." Bioresource Technology 85: 35-37.

Parsons, M. J. and G. D. Fenwick (1984). "Marine algae and a marine fungus from Open Bay Islands, Westland, New Zealand." New Zealand Journal of Botany 22: 425-432.

Peng, Y., Z. Chi, et al. (2010). " $\beta$-1,3-glucanase inhibits activity of the killer toxin produced by the marine-derived yeast Williopsis saturnus WC91-2." Marine Biotechnology 12(4): 479-485.

Phaff, H. J., E. M. Mrak, et al. (1952). "Yeasts isolated from shrimp." Mycologia 44(4): 431-451. 
Pitkaranta, M., T. Meklin, et al. (2008). "Analysis of fungal flora in indoor dust by ribosomal DNA sequence analysis, quantitative PCR, and culture." Applied and Environmental Microbiology 74(1): 233-244.

Raghukumar, C. (2002). Bioremediation of Coloured Pollutants by Terrestrial versus Facultative Marine Fungi. Fungi in Marine Environments. K. D. Hyde. Hong Kong, Fungal Diversity Press. 7: 317-344.

Raghukumar, C. (2008). "Marine fungal biotechnology: an ecological perspective." Fungal Diversity 31: 19-35.

Raspor, P. and J. Zupan (2006). Yeasts in Extreme Environments. Biodiversity and Ecophysiology of Yeasts. C. Rosa and G. Pétér. Germany, Springer-Verlag. 1: 371-417.

Reyesbecerril, M., I. Salinas, et al. (2008). "Oral delivery of live yeast Debaryomyces hansenii modulates the main innate immune parameters and the expression of immune-relevant genes in the gilthead seabream (Sparus aurata L.)." Fish \& Shellfish Immunology 25(6): 731-739.

Rheims, H., F. A. Rainey, et al. (1996). "A molecular approach to search for diversity among bacteria in the environment." Journal of Industrial Microbiology 17: 159-169.

Rhishipal, R. and R. Philip (1998). "Selection of marine yeasts for the generation of single cell protein from prawn-shell waste." Bioresource Technology 65: 255-256.

Roth, F. J., Jr., D. G. Ahearn, et al. (1962). "Ecology and taxonomy of yeasts isolated from various marine substrates." Limnology and Oceanography 7(2): 178-185.

Sajeevan, T., R. Philip, et al. (2006). "Immunostimulatory effect of a marine yeast Candida sake S165 in Fenneropenaeus indicus." Aquaculture 257(1-4): 150-155.

Sarri, S., P. Misaelides, et al. (2009). "Uranium removal from acidic aqueous solutions by Saccharomyces cerevisiae, Debaryomyces hansenii, Kluyveromyces marxianus and Candida colliculosa." Journal of Radioanalytical and Nuclear Chemistry 279(3): 709-711.

Schena, L., A. Ippolito, et al. (1999). "Genetic diversity and biocontrol activity of Aureobasidium pullulans isolates against postharvest rots." Postharvest Biology and Technology 17: 189-199.

Scorzetti, G., J. W. Fell, et al. (2002). "Systematics of basidiomycetous yeasts: a comparison of large subunit D1/D2 and internal transcribed spacer rDNA regions." FEMS Yeast Research 2: 495-517.

Seifert, K. A. (2009). "Progress towards DNA barcoding of fungi." Molecular Ecology Resources 9: 83-89.

Seki, H. and J. Fulton (1969). "Infection of marine copepods by Metschnikowia sp." Mycopathologia 38(1-2): 61-70. 
Serjeant, K., R. Tang, et al. (2008). "Yeasts associated with the New Zealand Nothofagus honeydew system." New Zealand Journal of Ecology 32(2): 209-213.

Seshadri, R. and J. M. Sieburth (1971). "Cultural estimation of yeasts on seaweeds." Applied Microbiology 22(4): 507-512.

Singh, P., C. Raghukumar, et al. (2010). "Phylogenetic diversity of culturable fungi from the deep-sea sediments of the Central Indian Basin and their growth characteristics." Fungal Diversity 40(1): 89-102.

Strouhal, M. (2003). "Electrochemical study of heavy metals and metallothionein in yeast Yarrowia lipolytica." Bioelectrochemistry 60(1-2): 29-36.

Tamura, K. and M. Nei (1993). "Estimation of the number of nucleotide substitutions in the control region of mitochondrial DNA in humans and chimpanzees." Molecular Biology and Evolution 10(3): 512-526.

Tanghe, A., B. Prior, et al. (2006). Yeast Responses to Stresses. Biodiversity and Ecophysiology of Yeasts. C. Rosa and G. Pétér. Germany, Springer-Verlag. 1: 175-195.

Taysi, I. and N. Van Uden (1964). "Occurrence and population densities of yeast species in an estuarine-marine area." Limnology and Oceanography 9(1): 42-45.

Valente, P., J. P. Ramos, et al. (1999). "Sequencing as a tool in yeast molecular taxonomy." Canadian Journal of Microbiology 45: 949-958.

Van Uden, N. (1960). "The occurrence of Candida and other yeasts in the intestinal tracts of animals." Annals of the New York Academy of Sciences 89: 59-68.

Van Uden, N. and R. Castelo Branco (1963). "Distribution and population densities of yeast species in Pacific water, air, animals, and kelp of southern California." Limnology and oceanography 8 .

Vishniac, H., S. (2006). Yeast Biodiversity in the Antarctic. Biodiversity and Ecophysiology of Yeasts. C. Rosa and G. Pétér. Germany, Springer-Verlag. 1: 419-440.

Wang, X., Z. Chi, et al. (2007). "A marine killer yeast against the pathogenic yeast strain in crab (Portunus trituberculatus) and an optimization of the toxin production." Microbiological Research 162(1): 77-85.

Wang, Y., Y. Bao, et al. (2008). "Biocontrol of Alternaria alternata on cherry tomato fruit by use of marine yeast Rhodosporidium paludigenum " International Journal of Food Microbiology 123(3): 234-239. 
Wang, Y., T. Yu, et al. (2010). "Biocontrol of postharvest gray mold of cherry tomatoes with the marine yeast Rhodosporidium paludigenum." Biological Control 53(2): 178-182.

Whitaker, R. J., D. W. Grogan, et al. (2003). "Geographic barriers isolate endemic populations of hyperthermophilic archaea." Science 301(5635): 976-978.

White, T. J., T. Bruns, et al. (1990). Amplification and Direct Sequencing of Fungal Ribosomal RNA Genes for Phylogenetics. PCR protocols: a guide to methods and applications. M. A. Innis. San Diego, Academic Press.

Wilson, I. M. (1960). "Marine fungi: a review of the present position." Proceedings of the Linnean Society of London 171(1): 53-70.

Yamasato, K., S. Goto, et al. (1974). "Yeasts from the Pacific Ocean." Journal of General and Applied Microbiology 20: 289-307.

Zalar, P., C. Gostincar, et al. (2008). "Redefinition of Aureobasidium pullulans and its varieties." Studies in Mycology 61(1): 21-38.

Zinjarde, S. S. and A. A. Pant (2002). "Hydrocarbon degraders from tropical marine environments." Marine Pollution Bulletin 44(2): 118-121. 